\title{
Four-loop non-singlet splitting functions in the planar limit and beyond
}

\author{
S. Moch, ${ }^{a}$ B. Ruijl, ${ }^{b, c}$ T. Ueda, ${ }^{b}$ J.A.M. Vermaseren ${ }^{b}$ and A. $\operatorname{Vogt}^{d}$ \\ ${ }^{a}$ II. Institute for Theoretical Physics, Hamburg University, \\ Luruper Chaussee 149, D-22761 Hamburg, Germany \\ ${ }^{b}$ Nikhef Theory Group, \\ Science Park 105, 1098 XG Amsterdam, The Netherlands \\ ${ }^{c}$ Leiden Centre of Data Science, Leiden University, \\ Niels Bohrweg 1, 2333 CA Leiden, The Netherlands \\ ${ }^{d}$ Department of Mathematical Sciences, University of Liverpool, \\ Liverpool L69 3BX, U.K. \\ E-mail: sven-olaf.moch@desy.de, benruijl@gmail.com, tueda@nikhef.nl, \\ Jos.Vermaseren@nikhef.nl, Andreas.Vogt@liverpool.ac.uk
}

ABSTRACT: We present the next-to-next-to-next-to-leading order $\left(\mathrm{N}^{3} \mathrm{LO}\right)$ contributions to the non-singlet splitting functions for both parton distribution and fragmentation functions in perturbative QCD. The exact expressions are derived for the terms contributing in the limit of a large number of colours. For the remaining contributions, approximations are provided that are sufficient for all collider-physics applications. From their threshold limits we derive analytical and high-accuracy numerical results, respectively, for all contributions to the four-loop cusp anomalous dimension for quarks, including the terms proportional to quartic Casimir operators. We briefly illustrate the numerical size of the four-loop corrections, and the remarkable renormalization-scale stability of the $\mathrm{N}^{3} \mathrm{LO}$ results, for the evolution of the non-singlet parton distribution and the fragmentation functions. Our results appear to provide a first point of contact of four-loop QCD calculations and the so-called wrapping corrections to anomalous dimensions in $\mathcal{N}=4$ super Yang-Mills theory.

KEYwords: Perturbative QCD, Resummation

ArXiv EPrint: 1707.08315 


\section{Contents}

1 Introduction $\quad 1$

2 Theoretical framework and calculations 3

3 Results in $N$-space $\quad 10$

4 Results in $x$-space $\quad 15$

5 Numerical implications $\quad 27$

6 The time-like case $\quad 30$

$\begin{array}{lll}7 & \text { Summary and outlook } & 34\end{array}$

$\begin{array}{ll}\text { A Feynman rules } & 38\end{array}$

B Mellin moments at four loops $\quad 41$

$\begin{array}{ll}\text { C Time-like splitting function } & 56\end{array}$

D The complete $\zeta_{5}$ contributions $\quad 72$

\section{Introduction}

Within the gauge theory of the strong interaction, Quantum Chromodynamics (QCD), the precision of theory predictions for hard reactions at colliders crucially depends on our knowledge of hadronic matrix elements for the description of the long-distance hadronic degrees of freedom, once the hard-interaction part due to short-distance physics has been separated by means of QCD factorization. For scattering reactions with initial-state protons the relevant matrix elements are given by the well-known parton distribution functions (PDFs) of the proton, which provide information about the fractions of the proton's longitudinal momentum carried by the partons.

The dependence of these PDFs on the scale $Q^{2}$ is generated by evolution equations for the corresponding local operator matrix elements (OMEs). The relevant anomalous dimensions as functions of the Mellin moment $N$, or splitting functions as functions of the momentum fraction $x$, can be computed order by order in perturbative QCD. The corresponding one- and two-loop results have been known since long [1-13]. The current precision is at the three-loop level $[14,15]$ — see refs. [16-19] for partial recalculations of these results - i.e., at the next-to-next-to-leading order (NNLO), which is nowadays the 
accepted standard for analyses of PDFs [20] and forms the backbone of precision predictions at the Large Hadron Collider (LHC).

However, computations for a number key observables at hadron colliders have been performed even at next-to-next-to-next-to-leading order $\left(\mathrm{N}^{3} \mathrm{LO}\right)$, including the cross section for Higgs-boson production in gluon-gluon fusion [21] and structure functions in deepinelastic scattering (DIS) [22-25]. The latter results have also found an application in predicting Higgs-boson production in vector-boson fusion at the LHC [26]. Due to QCD factorization, the resulting predictions carry a residual uncertainty and dependence on the factorization scheme due to the missing $\mathrm{N}^{3} \mathrm{LO}$ (i.e., four-loop) splitting functions. This situation motivates the computation of the QCD splitting functions at four loops. First steps in this direction have already been taken in refs. [27-31] at low $N$, and in ref. [32] where large- $n_{f}$ contributions have been derived at all $N$.

In the present article, we address the splitting functions for the non-singlet quark evolution equations at four loops in QCD. We use Forcer [33], a Form [34-36] program for four-loop massless propagators, to compute the anomalous dimensions at fixed integer values of the Mellin variable $N$. In the planar limit, i.e., for large $n_{c}$ for a general colour $\mathrm{SU}\left(n_{c}\right)$ gauge group, the exact four-loop results for moments up to $N=20$ turn out to be sufficient to find and validate the analytic expressions as functions of $N$ in terms of harmonic sums $[37,38]$ by LLL-based techniques $[39,42]^{1}$ for solving systems of Diophantine equations. Such an approach has been used for anomalous dimensions at the three-and four-loop level before, cf. refs. [32, 43, 44]. Our analytic results in the threshold limit $x \rightarrow 1$ $(N \rightarrow \infty)$ include the (light-like) four-loop cusp anomalous dimension, see ref. [45], which has also been obtained in refs. [46, 47] by different means.

Beyond the large- $n_{c}$ limit, we have computed the moments up to $N=16$ for a general gauge group. These results are insufficient for a reconstruction of the analytic all- $N$ results. They can be used, though, to obtain approximations for the four-loop splitting functions including $x$-dependent estimates of their residual uncertainties, see, e.g., earlier work at the three-loop level [48-50]. The approximations presented below are sufficiently accurate for the evolution of non-singlet PDFs down to small $x$, and include numerical results for the non-planar contributions to the four-loop cusp anomalous dimension that are sufficiently precise for phenomenological applications.

For processes with identified hadrons in the final state, QCD factorization requires fragmentation functions (FFs) that account for the physics of hadronization at long distances. Completely analogous to PDFs, the scale dependence of FFs can be computed within perturbative QCD. However, in contrast to the case of initial state hadrons, where the evolution equations for the scale-dependence of the PDFs are controlled by space-like kinematics, $Q^{2} \leq 0$, the scale evolution of the FFs with $Q^{2} \geq 0$ requires the so-called timelike splitting functions. These functions are known completely at two loops [9-11, 51-53], see also refs. $[54,55]$. The three-loop corrections have been obtained in refs. [56-58] up to a phenomenologically irrelevant small uncertainty in the result for the time-like NNLO quarkgluon splitting function. First NNLO analyses of FFs have been performed recently [59, 60].

\footnotetext{
${ }^{1}$ See also ref. [40], summarized in [41], pg. 16.
} 
The three-loop results in refs. [56-58] have been derived using well-known relations between space- and time-like kinematics, i.e., the Drell-Yan-Levy relation for the analytic continuation in energy $q^{2} \rightarrow-q^{2}$ and the Gribov-Lipatov relation in $x$-space [61, 62], see also refs. [63, 64], and generalizations based on conformal symmetry yielding a universal reciprocity-respecting evolution kernel [65-67]. Exploiting these relations, it is possible to use (space-like) DIS results to predict (time-like) cross sections for single-particle inclusive electron-positron annihilation. Thus, we are able to present here also the flavour non-singlet evolution equations for FFs at four loops in QCD.

This article is organized as follows. In section 2 we specify our notations and present the theoretical framework for obtaining our results. In particular we address the basis of non-singlet operators, their renormalization and the respective anomalous dimensions. We sketch the work-flow of the perturbative computation up to four loops, list all colour factors to this order and discuss general and end-point properties of the anomalous dimensions and splitting functions.

In section 3 we present the results of our fixed- $N$ diagram calculations of the fourloop non-singlet anomalous dimensions and their all- $N$ generalization in the large- $n_{c}$ limit. We discuss the large- $N$ behaviour of the latter which includes the four-loop cusp anomalous dimension. The $x$-space counterparts of these anomalous dimensions, i.e., the splitting functions, are addressed in section 4 . We present the exact formulae and compact parametrizations for the large- $n_{c}$ splitting functions, and approximate expressions for all cases that cannot be obtained exactly for now.

Two important applications of these results are presented in section 5: we present high-accuracy numerical results for large- $x$ coefficients, in particular the four-loop cusp anomalous dimension in $\mathrm{QCD}$, and illustrate the $\mathrm{N}^{3} \mathrm{LO}$ evolution of all three types of nonsinglet quark distributions. The $\mathrm{N}^{3} \mathrm{LO}$ non-singlet evolution is extended to the 'time-like' case of final-state fragmentation functions in section 6 . We summarize our main results and provide a brief outlook in section 7 .

The appendices contain the Feynman rules in appendix A, the exact results for the anomalous dimensions at $1 \leq N \leq 16$ at four loops in appendix $\mathrm{B}$, and the analytic expression for the difference of the time-like and space-like four-loop splitting functions in appendix C. Finally appendix D provides the complete all- $N$ result for the terms with $\zeta_{5}$, which may be of theoretical interest.

\section{Theoretical framework and calculations}

The standard set of spin- $N$ twist-two irreducible flavour non-singlet quark operators is given by

$$
O_{\left\{\mu_{1}, \ldots, \mu_{N}\right\}}^{\mathrm{ns}}=\bar{\psi} \lambda^{\alpha} \gamma_{\left\{\mu_{1}\right.} D_{\mu_{2}} \ldots D_{\left.\mu_{N}\right\}} \psi, \quad \alpha=3,8, \ldots,\left(n_{f}^{2}-1\right),
$$

where $\psi$ represents the quark field, $D_{\mu}=\partial_{\mu}-\mathrm{i} g A_{\mu}$ the covariant derivative, and $\lambda^{\alpha}$ the diagonal generators of the flavour group $\mathrm{SU}\left(n_{f}\right)$. It is understood in eq. (2.1) that the symmetric and traceless part is taken with respect to the Lorentz indices $\mu_{i}$ in the curly brackets. 


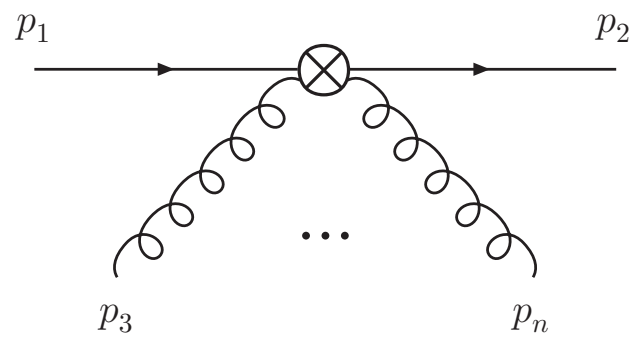

Figure 1. Vertices with additional gluons arising from the operators $O_{\left\{\mu_{1}, \ldots, \mu_{N}\right\}}^{\text {ns }}$ in perturbative QCD. Vertices with up to $L$ gluons need to be considered at $L$ loops at Mellin moments $N>L$.

We consider (spin-averaged) matrix elements of these operators (OMEs), specifically

$$
\left\langle p_{1}\left|O_{\left\{\mu_{1}, \ldots, \mu_{N}\right\}}^{\mathrm{ns}}\right| p_{2}\right\rangle,
$$

for external quark (or anti-quark) fields with momenta $p_{1}$ and $p_{2}$. The operators $O^{\text {ns }}$ in eq. (2.2) are contracted with tensors of rank $N$,

$$
\Delta^{\mu_{1}} \ldots \Delta^{\mu_{N}}
$$

where $\Delta$ is a light-like vector, $\Delta^{2}=0$. In the present context we need to compute OMEs of renormalized operators with zero momentum flow through the operator vertex, thus $p_{1}=p_{2}=p$ in eq. (2.2) for the (off-shell, $p^{2} \neq 0$ ) momenta of the external (anti-) quarks,

$$
\left[A^{\mathrm{ns}}\right](N)=\Delta^{\mu_{1}} \ldots \Delta^{\mu_{N}}\left\langle p\left|\left[O^{\mathrm{ns}}\right]_{\left\{\mu_{1}, \ldots, \mu_{N}\right\}}\right| p\right\rangle .
$$

Here and below we use square brackets [... to denote renormalized operators (in a minimal subtraction scheme [68, 69] of dimensional regularization [70, 71]). This reduces the vertex diagrams for the OMEs to quark two-point functions and, therefore, the computational complexity to propagator-type diagrams. The perturbative expansion of the operator in eq. (2.1) contracted with eq. (2.3) generates vertices with additional gluons as depicted in figure 1. The current four-loop calculation requires up to four additional gluons. The corresponding Feynman rules are presented in appendix A, see refs. [5, 72] for earlier calculations at two- and three-loop accuracy.

In order to derive the anomalous dimensions for the scale dependence of the non-singlet PDFs we need to perform the renormalization of those operators $O^{\text {ns }}$, which proceeds multiplicatively as

$$
\left[O^{\mathrm{ns}}\right]=Z_{\mathrm{ns}} O^{\mathrm{ns}}
$$

The anomalous dimensions $\gamma_{\mathrm{ns}}$ governing the scale dependence of these operators,

$$
\frac{d}{d \ln \mu^{2}}\left[O^{\mathrm{ns}}\right]=-\gamma_{\mathrm{ns}}\left[O^{\mathrm{ns}}\right]
$$

are connected to the factors $Z_{\mathrm{ns}}$ in eq. (2.5) by

$$
\gamma_{\mathrm{ns}}=-\left(\frac{d}{d \ln \mu^{2}} Z_{\mathrm{ns}}\right) Z_{\mathrm{ns}}^{-1} .
$$


All flavour differences of quark-anti-quark sums $(+)$ and differences $(-)$ evolve in with the same anomalous dimensions $\gamma_{\mathrm{ns}}^{+}(N)$ and $\gamma_{\mathrm{ns}}^{-}(N)$, and the total valence distribution with $\gamma_{\text {ns }}^{\mathrm{v}}(N)=\gamma_{\mathrm{ns}}^{-}(N)+\gamma_{\mathrm{ns}}^{\mathrm{s}}(N)$, see, e.g., ref. [14]. These quantities are related to the corresponding splitting functions $P_{\mathrm{ns}}^{ \pm}(x)$ and $P_{\mathrm{ns}}^{\mathrm{s}}(x)$ by a Mellin transform,

$$
\gamma_{\mathrm{ns}}(N)=-\int_{0}^{1} d x x^{N-1} P_{\mathrm{ns}}(x)
$$

where the relative sign is a standard convention. In perturbation theory these quantities can be expanded in powers of the strong coupling constant $\alpha_{\mathrm{s}}$. Here and below we normalize $a_{\mathrm{s}} \equiv \alpha_{\mathrm{s}} /(4 \pi)$, so that up to four loops

$$
\gamma_{\mathrm{ns}}(N)=a_{\mathrm{s}} \gamma_{\mathrm{ns}}^{(0)}(N)+a_{\mathrm{s}}^{2} \gamma_{\mathrm{ns}}^{(1)}(N)+a_{\mathrm{s}}^{3} \gamma_{\mathrm{ns}}^{(2)}(N)+a_{\mathrm{s}}^{4} \gamma_{\mathrm{ns}}^{(3)}(N),
$$

and similarly for the splitting functions $P_{\mathrm{ns}}(x)$ and other quantities. The first-order quantity $\gamma_{\mathrm{ns}}^{(0)}$ is the same for all three cases given above. $\gamma_{\mathrm{ns}}^{+}$and $\gamma_{\mathrm{ns}}^{-}$differ at order $a_{\mathrm{s}}^{2}$, and a non-vanishing flavour-independent ('sea') contribution $\gamma_{\mathrm{ns}}^{\mathrm{s}}$ occurs at order $a_{\mathrm{s}}^{3}$ for the first time [14]. The fourth-order contributions $\gamma_{\mathrm{ns}}^{(3)}(N)$ to all three quantities are addressed in the present article.

The actual computation follows a well-established production chain. The Feynman diagrams for the OMEs in eq. (2.2) are generated up to four loops using QGRAF [73]. The latest version [74] of the symbolic manipulation program Form [34, 35] and its multithreaded version TFORM [36] are used for all further steps. The QGRAF output is processed by a program that assigns the topology and computes the colour factor using the code of ref. [75]; the group invariants occurring in the present case are listed in table 1. Diagrams of the same topology and colour factor are combined to meta diagrams for computational efficiency, where lower-order self-energy insertions are treated as described in ref. [76]. Considering all color factors, this procedure leads to 1 one-loop, 7 two-loop, 53 three-loop and 650 four-loop meta diagrams for $\gamma_{\text {ns }}^{ \pm}$; and 1 three-loop and 29 four-loop meta diagrams for $\gamma_{\text {ns }}^{\mathrm{s}}$. For comparison: the output of QGRAF consists of 15901 four-loop diagrams. The running of the meta diagrams is managed using the database program Minos [77].

The diagram calculations are done in dimensional regularization [70, 71] with the FORCER program [33] which was already used for the $N \leq 6$ and high- $n_{f}$ computations in refs. [31, 32]. Our agreement (after renormalization, see below) with those results, which were obtained in a different theoretical framework, provides a strong check of our present setup. The FORCER program itself has been validated in calculations of the four-loop renormalization of Yang-Mills theories to all powers of the gauge parameter, see ref. [79], and has recently been applied - together with the algorithms for the $R^{*}$ operation $[80,81]$ developed in ref. [82] — in five-loop computations of the beta function, Higgs-boson decays to hadrons and the $R$-ratio in $e^{+} e^{-}$-annihilation in refs. [83, 84].

The bare results $A^{\text {ns }}$ for the OMEs in eq. (2.4) obtained in this way are then subject to renormalization which we perform in the standard modified minimal subtraction scheme $\overline{\mathrm{MS}}[68,69]$. In this scheme and in $D=4-2 \epsilon$ dimensions the strong coupling $\alpha_{\mathrm{s}}$ evolves according to

$$
\frac{d}{d \ln \mu^{2}} \frac{\alpha_{\mathrm{s}}}{4 \pi} \equiv \frac{d a_{\mathrm{s}}}{d \ln \mu^{2}}=\beta\left(a_{\mathrm{s}}\right)=-\varepsilon a_{\mathrm{s}}-\beta_{0} a_{\mathrm{s}}^{2}-\beta_{1} a_{\mathrm{s}}^{3}-\beta_{2} a_{\mathrm{s}}^{4}-\ldots
$$




\begin{tabular}{|cccc|}
\hline & & $\mathrm{SU}\left(n_{c}\right)$ & $\mathrm{QCD}$ \\
\hline$C_{A}$ & $\mathrm{ca}$ & $n_{c}$ & 3 \\
$C_{F}$ & $\mathrm{cf}$ & $\frac{n_{c}^{2}-1}{2 n_{c}}$ & $\frac{4}{3}$ \\
$\frac{d^{a b c} d_{a b c}}{N_{R}}$ & {$[\mathrm{dabc} \wedge 2 / \mathrm{nr}]$} & $\frac{\left(n_{c}^{2}-1\right)\left(n_{c}^{2}-4\right)}{16 n_{c}^{2}}$ & $\frac{5}{18}$ \\
$\frac{d_{F}^{a b c d} d_{A}^{a b c d}}{N_{R}}$ & {$[\mathrm{~d} 4 \mathrm{RA} / \mathrm{nr}]$} & $\frac{\left(n_{c}^{2}+6\right)\left(n_{c}^{2}-1\right)}{48}$ & $\frac{5}{2}$ \\
$\frac{d_{F}^{a b c d} d_{F}^{a b c d}}{N_{R}}$ & {$[\mathrm{~d} 4 \mathrm{RR} / \mathrm{nr}]$} & $\frac{\left(n_{c}^{4}-6 n_{c}^{2}+18\right)\left(n_{c}^{2}-1\right)}{96 n_{c}^{3}}$ & $\frac{5}{36}$ \\
\hline
\end{tabular}

Table 1. The colour factors for non-singlet OMEs up to four loops with their numerical values in $\mathrm{SU}\left(n_{c}\right)$ with $N_{R}=n_{c}$ and QCD, see also ref. [78] for a discussion on the normalization of $d^{a b c} d_{a b c}$. The second column gives the notations of the result files, which are distributed with this article on https://arxiv.org. As in many other articles, we suppress the colour factor $T_{F}(=1 / 2$ in $\left.\mathrm{SU}\left(n_{c}\right)\right)$ which can be readily re-instated.

where $\beta\left(a_{\mathrm{s}}\right)$ denotes the usual four-dimensional beta function in QCD, with coefficients $\beta_{0}=11 / 3 C_{A}-2 / 3 n_{f}$ etc, and $n_{f}$ represents the number of active quark flavours.

Using eq. (2.5) the renormalized OMEs $\left[A^{\mathrm{ns}}\right]$ are obtained by

$$
\left[A^{\mathrm{ns}}\right](N)=Z_{\psi} Z_{\mathrm{ns}}(N) A^{\mathrm{ns}}(N),
$$

where we have made all dependences on $N$ explicit. The factor $Z_{\psi}$ denotes the quark wave function renormalization constant accounting for the external quarks field with off-shell momenta in eq. (2.4), see, for instance ref. [79]. Unlike $Z_{\mathrm{ns}}$, the quantities $Z_{\psi}$ and $A^{\text {ns }}$ are gauge-dependent, hence also the renormalization constant $Z_{\xi}$ of the gauge parameter is required.

The resulting operator renormalization factors $Z_{\mathrm{ns}}$ in eq. (2.5) can be expressed as a Laurent series in $\varepsilon$ as

$$
\begin{aligned}
& Z_{\mathrm{ns}}=1+a_{\mathrm{s}}\left\{\frac{1}{\epsilon} \gamma_{\mathrm{ns}}^{(0)}\right\}+a_{\mathrm{s}}^{2}\left\{\frac{1}{\epsilon^{2}}\left(\frac{1}{2}\left(\gamma_{\mathrm{ns}}^{(0)}\right)^{2}-\frac{1}{2} \gamma_{\mathrm{ns}}^{(0)} \beta_{0}\right)+\frac{1}{2 \epsilon} \gamma_{\mathrm{ns}}^{(1)}\right\} \\
& +a_{\mathrm{s}}^{3}\left\{\frac{1}{\epsilon^{3}}\left(\frac{1}{6}\left(\gamma_{\mathrm{ns}}^{(0)}\right)^{3}-\frac{1}{2} \beta_{0}\left(\gamma_{\mathrm{ns}}^{(0)}\right)^{2}+\frac{1}{3} \beta_{0}^{2} \gamma_{\mathrm{ns}}^{(0)}\right)+\frac{1}{\epsilon^{2}}\left(\frac{1}{2} \gamma_{\mathrm{ns}}^{(1)} \gamma_{\mathrm{ns}}^{(0)}-\frac{1}{3} \beta_{0} \gamma_{\mathrm{ns}}^{(1)}-\frac{1}{3} \beta_{1} \gamma_{\mathrm{ns}}^{(0)}\right)\right. \\
& \left.+\frac{1}{3 \epsilon} \gamma_{\mathrm{ns}}^{(2)}\right\}+a_{\mathrm{s}}^{4}\left\{\frac{1}{\epsilon^{4}}\left(\frac{1}{24}\left(\gamma_{\mathrm{ns}}^{(0)}\right)^{4}-\frac{1}{4} \beta_{0}\left(\gamma_{\mathrm{ns}}^{(0)}\right)^{3}+\frac{11}{24} \beta_{0}^{2}\left(\gamma_{\mathrm{ns}}^{(0)}\right)^{2}-\frac{1}{4} \beta_{0}^{3} \gamma_{\mathrm{ns}}^{(0)}\right)\right. \\
& +\frac{1}{\epsilon^{3}}\left(\frac{1}{4} \gamma_{\mathrm{ns}}^{(1)}\left(\gamma_{\mathrm{ns}}^{(0)}\right)^{2}-\frac{7}{12} \beta_{0} \gamma_{\mathrm{ns}}^{(1)} \gamma_{\mathrm{ns}}^{(0)}+\frac{1}{4} \beta_{0}^{2} \gamma_{\mathrm{ns}}^{(1)}+\frac{1}{2} \beta_{0} \beta_{1} \gamma_{\mathrm{ns}}^{(0)}-\frac{1}{3} \beta_{1}\left(\gamma_{\mathrm{ns}}^{(0)}\right)^{2}\right) \\
& \left.+\frac{1}{\epsilon^{2}}\left(\frac{1}{3} \gamma_{\mathrm{ns}}^{(2)} \gamma_{\mathrm{ns}}^{(0)}+\frac{1}{8}\left(\gamma_{\mathrm{ns}}^{(1)}\right)^{2}-\frac{1}{4} \gamma_{\mathrm{ns}}^{(2)} \beta_{0}-\frac{1}{4} \beta_{1} \gamma_{\mathrm{ns}}^{(1)}-\frac{1}{4} \beta_{2} \gamma_{\mathrm{ns}}^{(0)}\right)+\frac{1}{4 \epsilon} \gamma_{\mathrm{ns}}^{(3)}\right\} .
\end{aligned}
$$

In this manner, the anomalous dimensions $\gamma_{\mathrm{ns}}$ have been computed for a general gauge group at $1 \leq N \leq 16$, i.e, $\gamma_{\mathrm{ns}}^{+}$at even $N$ and $\gamma_{\mathrm{ns}}^{-, \mathrm{s}}$ at odd $N$. The exact results are listed 
in appendix B; numerical values for QCD can be found in section 3. The hardest (nonplanar) diagrams do not contribute in the limit of a large number of colours $n_{c}$, where the functions $\gamma_{\text {ns }}^{+}(N)$ and $\gamma_{\text {ns }}^{-}(N)$ are identical, as it is evident from diagrammatical analyses and the known $x$-space expressions for $P_{\mathrm{ns}}^{ \pm}(x)$, see refs. [14, 32, 66, 85]. Consequently we were able to obtain the even- $N$ and odd- $N$ values of the large- $n_{c}$ anomalous dimension, which is structurally simpler than full QCD results, even up to $N=20$.

So far, fixed- $N$ values of anomalous dimensions have been found to be fractions of (large) integer numbers, multiplied at most by values $\zeta_{3} \ldots \zeta_{2 L-3}$ of the Riemann zetafunction at $L$ loops. The denominator structure of the fractions suggests analytic all$N$ expressions in terms of harmonic sums [37, 38] up to weight $2 L-1$. Assuming no numerator- $N$ terms, cf. refs. [22-24], the most complicated parts (without a factor $\zeta_{n}$ ) of the non-singlet anomalous dimensions at $n$ loops read

$$
\gamma_{\mathrm{ns}}^{(n)}(N)=\sum_{w=0}^{2 n+1} c_{00 w} S_{w}(N)+\sum_{a} \sum_{k=1}^{2 n+1} \sum_{w=0}^{2 n+1-k} c_{a k w} D_{a}^{k} S_{w}(N),
$$

where $D_{a}^{k}$ are simple denominators,

$$
D_{a}^{k}=(N+a)^{-k}
$$

and $S_{w}(N)$ is a shorthand for all harmonic sums of a given weight $w$ with $S_{0}(N) \equiv 1$. The calculated moments suggest $a=0,1$ for $\gamma_{\mathrm{ns}}^{(3) \pm}(N)$, as at three loops [14] and for the $n_{f}^{2}$ and $n_{f}^{3}$ four-loop contributions [32]. The function $\gamma_{\mathrm{ns}}^{\mathrm{s}}(N)$, on the other hand, includes terms with $a=-1$ and $a=2$.

The functions $\gamma_{\mathrm{ns}}^{(3)}(N)$ contain harmonic sums up to weight $w=7$, hence the ansatz (2.13) includes far too many unknown coefficients $c_{a k w}$ for a direct determination from the (small) number of calculated moments. However, these coefficients are integer modulo some predictable powers of 2 and 3. Therefore the systems of equations derived from eq. (2.13) can be turned into Diophantine systems which require far fewer equations than unknowns and which can be solved by LLL-based techniques [39-42]. This approach has been successfully applied before in refs. [32, 43, 44].

In this context it is crucial to constrain eq. (2.13) as far as possible based on general properties of the anomalous dimensions $\gamma_{\mathrm{ns}}(N)$. Here three issues are worth pointing out. First, the functional forms of the $\gamma_{\mathrm{ns}}(N)$ are (conjectured to be) constrained by 'self-tuning' [66, 67],

$$
\left.\gamma_{\mathrm{ns}}(N)=\gamma_{\mathrm{u}}\left(N+\sigma \gamma_{\mathrm{ns}}(N)-\beta\left(a_{\mathrm{s}}\right) / a_{\mathrm{s}}\right)\right)
$$

where $\sigma=-1(+1)$ for the space-like (time-like) anomalous dimensions, and the nonsinglet universal evolution kernel $\gamma_{\mathrm{u}}$ is reciprocity-respecting (RR), i.e., invariant under the replacement $N \rightarrow(1-N)$. By expanding the r.h.s. of eq. (2.15) about $N$ and inserting the perturbation series of all quantities involved, $\gamma_{u}$ can be expressed in terms of the $\overline{\mathrm{MS}}$ anomalous dimensions, see also ref. [86]. Expressing the latter in terms of $\gamma_{0}=$ $\gamma_{\mathrm{ns}}^{S(n)}=\gamma_{\mathrm{ns}}^{T(n)}$ and the average of the space-like and time-like expansion coefficients $\bar{\gamma}_{n}=$ 
$\frac{1}{2}\left(\gamma_{\mathrm{ns}}^{S(n)}+\gamma_{\mathrm{ns}}^{T(n)}\right)$, one arrives at

$$
\begin{aligned}
\gamma_{\mathrm{u}}= & a_{\mathrm{s}} \gamma_{0}+a_{\mathrm{s}}^{2}\left(\bar{\gamma}_{1}-\beta_{0} d_{N} \gamma_{0}\right)+a_{\mathrm{s}}^{3}\left(\bar{\gamma}_{2}-\frac{1}{6} d_{N}^{2} \gamma_{0}^{3}-\beta_{1} d_{N} \gamma_{0}-\beta_{0} d_{N} \bar{\gamma}_{1}+\frac{1}{2} \beta_{0}^{2} d_{N}^{2} \gamma_{0}\right) \\
+ & a_{\mathrm{s}}^{4}\left(\bar{\gamma}_{3}-\frac{1}{2} d_{N}^{2}\left(\gamma_{0}^{2} \bar{\gamma}_{1}\right)-\beta_{2} d_{N} \gamma_{0}-\beta_{1} d_{N} \bar{\gamma}_{1}-\beta_{0} d_{N} \bar{\gamma}_{2}+\frac{1}{6} \beta_{0} d_{N}^{3} \gamma_{0}^{3}+\beta_{0} \beta_{1} d_{N}^{2} \gamma_{0}\right. \\
& \left.+\frac{1}{2} \beta_{0}^{2} d_{N}^{2} \bar{\gamma}_{1}-\frac{1}{6} \beta_{0}^{3} d_{N}^{3} \gamma_{0}\right)+\mathcal{O}\left(a_{\mathrm{s}}^{5}\right)
\end{aligned}
$$

where we have used the abbreviation $d_{N}=d / d N$ and suppressed the $N$-dependences for brevity. A convenient way to take these derivatives is via inverse Mellin transforms to $x$-space, where the multiplication with $\ln ^{n} x$ corresponds to the $N$-space operator $d^{n} / d N^{n}$, and Mellin transforms of the result. The required manipulations can be readily performed using algorithms for harmonic sums, harmonic polylogarithms and their (inverse) Mellin transformations $[37,87,88]$ which have been implemented in publicly available Form packages described in ref. [34].

Since the difference between the time-like and space-like anomalous dimensions is known to four loops, eq. (2.13) can be applied to the RR quantity $\gamma_{\mathrm{u}}^{(3)}$ instead of $\gamma_{\mathrm{ns}}^{(3)}$. This implies that the denominators $1 / N$ and $1 /(N+1)$ can only enter in the combination $1 /(N(N+1))$, and that only $\mathrm{RR}$ (combinations of) harmonic sums occur, see refs. [89, 90], which reduces the number of sums at weight $w$ from $2 \cdot 3^{w-1}$ to $2^{w-1}$. Assuming that only powers of $1 /(N+1)$ enter in addition, the total number of basis functions in eq. (2.13) up to weight $w$ is $2^{w+1}-1$, e.g., 255 for $w=7$. Even taking account end-point constraints, see below, this is a prohibitively large number for now.

Second, the identical leading- $n_{c}$ terms of $\gamma_{\text {ns }}^{ \pm}(N)$ contain only non-alternating harmonic sums, i.e., only positive indices in eq. (3.4). This reduces the number of RR sums of weight $w$ to the Fibonacci number $F(w)$, i.e., 1, 1, 2, 3, 5, 8, 13 for $w=1$ to $w=7$, as can be seen by counting the number of binomial harmonic sums at weight $w$ [89]. Considering all combinations with additional powers of the weight- 1 object $1 /(N(N+1)$, the total number of functions up to weight $w$ in eq. (2.13) amounts to $F(w+4)-2$, e.g., 87 for $w=7$.

The third and final point is that the $N \rightarrow \infty$ (large- $x)$ and $N \rightarrow 0$ (small- $x$ ) limits of the anomalous dimensions (splitting functions) provide a substantial number of constraints. If one disregards terms of order $\mathcal{O}\left(1 / N^{2}\right)$ for $N \rightarrow \infty$, then all three non-singlet anomalous dimensions $\gamma_{\mathrm{ns}}^{a}(N), a=+,-, \mathrm{v}$, are identical and given by [65] $\left(\gamma_{e}\right.$ is the Euler-Mascheroni constant)

$$
\gamma_{\mathrm{ns}}^{(n-1)}(N)=A_{n}\left(\ln N+\gamma_{e}\right)-B_{n}+C_{n}\left(\ln N+\gamma_{e}\right) N^{-1}-\left(\widetilde{D}_{n}-\frac{1}{2} A_{n}\right) N^{-1}
$$

Here the coefficients $A_{n}$ - the $n$-loop (light-like) cusp anomalous dimension - and $B_{n}$ provide genuine $n$-loop information. The coefficients $C_{n}$ and $\widetilde{D}_{n}$, on the other hand, can be expressed in terms of lower-order information (see eqs. (3.10) and (3.11) below). This and the absence of second and higher powers of $\ln N$ in eq. (2.17), and similar if less stringent constraints on $N^{-k} \ln N$ terms with $k>1$, provide a substantial number of constraints on the coefficients in eq. (2.13). 
The small- $x$ expansion of the splitting function $P_{\mathrm{ns}}^{(n)}(x)$ shows a double-logarithmic enhancement, i.e., there are contributions of the form $x^{a} \ln ^{b} x$ with $a>0$ and $b \leq 2 n$. The leading-logarithmic (LL) contributions to $P_{\mathrm{ns}}^{ \pm}$have been known to all orders for a long time [91, 92]. This resummation has been extended to next-to-next-to-logarithmic accuracy for the $x^{2 k} \ln ^{b} x$ contributions to $P_{\mathrm{ns}}^{+}$and the $x^{2 k+1} \ln ^{b} x$ contributions to $P_{\mathrm{ns}}^{-}$ at all $k \geq 0$ [93, 94]. The formal structure of these results is analogous to their time-like counterparts $[95,96]$, but the numerical pattern is completely different such that the spacelike resummation is of no direct phenomenological use. The functions $P_{\mathrm{ns}}^{+}(x)$ and $P_{\mathrm{ns}}^{-}(x)$ are the same in the large- $n_{c}$ limit, hence in this case the small- $x$ resummation constrains the coefficients contributing to

$$
x^{a} \ln ^{b} x \quad \text { for } \quad a \geq 0 \text { and } \quad 4 \leq b \leq 6 .
$$

An alternative approach to the limit $N \rightarrow 0$, i.e., the small- $x$ logarithms for $a=0$, has been pursued in ref. [97]. In the large- $n_{c}$ limit, the generalization

$$
\gamma_{\mathrm{ns}}\left(N, a_{\mathrm{s}}\right) \cdot\left(\gamma_{\mathrm{ns}}\left(N, a_{\mathrm{s}}\right)+N-\beta\left(a_{\mathrm{s}}\right) / a_{\mathrm{s}}\right)=O(1)
$$

of the LL relation in refs. [91, 92] correctly (re-) produces all $a=0$ small- $x$ logarithms obtained in refs. [14, 32, 93], after correcting typos in eqs. (25) and (26) of ref. [97]. Hence we can assume that eq. (2.19) is also correct for the $n_{f}^{0}$ and $n_{f}^{1}$ four-loop contributions at large $n_{c}$.

Together these relations comprise 18 large- $N$ and 28 small- $x$ constraints for the $n_{f}^{0}$ terms at four loops eliminating more than half of the 87 free parameters of the $w=7$ large- $n_{c}$ ansatz, after which it is possible to solve the remaining system of Diophantine equation using the moments $N=1, \ldots, 18$ with the program $\operatorname{axb}()$ of the CALC package [42]. The resulting analytic expressions for $\gamma_{\text {ns }}^{(3)}(N)$ agree with the result of the diagram calculations at $N=19$ and $N=20$. This agreement renders it extremely likely - although, of course, not mathematically certain - that these results (and, therefore, the above structural conjectures and features used in their derivation) are correct.

As mentioned above, present information and understanding appears not to be sufficient for extending these analytic results beyond the large- $n_{c}$ contributions for the $n_{f}^{0}$ and $n_{f}^{1}$ parts of $\gamma_{\mathrm{ns}}^{(3) a}(N)$ for any $a=+,-$, s. For the remaining functions we resort to $x$-space approximations based on the first eight even- $N$ or odd- $N$ moments supplemented by the large- $x$ and small- $x$ constraints discussed above. These approximations and their error estimates can be constructed in the same manner as those for the three-loop splitting functions in refs. [48-50]. The present results are more accurate, though, due to the higher number of available moments and the improved understanding of the end-point limits. The fact that the large- $N$ limit (2.17) includes only the two free parameters $A_{4}$ and $B_{4}$, in particular, results in a high accuracy of these coefficients which are relevant also in the context of the soft-gluon exponentiation, see refs. [98-101] and references therein, and beyond. 


\section{Results in $N$-space}

We start presenting our results by writing down the moments to $N=16$ of the non-singlet four-loop anomalous dimensions for QCD in a numerical form. The exact results for a general gauge group with one set of fermions can be found in appendix B. For $\gamma_{\mathrm{ns}}^{(3) \pm}(N)$ we separately display the leading (subscript $L$ ) and non-leading (subscript $N$ ) contributions in the large- $n_{c}$ limit of $\mathrm{SU}\left(n_{c}\right)$ at $n_{c}=3$. The former correspond to the colour factors $C_{F} n_{c}^{3-k} n_{f}^{k}$. The latter collect all other terms, which are suppressed by two or more powers of $n_{c}$, cf. table 1 above.

The first eight even- $N$ values of $\gamma_{\mathrm{ns}}^{(3)+}(N)$, normalized as in eq. (2.9) above - division by $2.5 \cdot 10^{4}$ provides an approximate conversion to an expansion in $\alpha_{\mathrm{s}}$ - are given by

$$
\begin{aligned}
\gamma_{\mathrm{ns}}^{(3)+}(2)= & 15079.979904_{L}+1583.245584_{N}-\left(3610.4544273_{L}+137.0956130_{N}\right) n_{f} \\
& +\left(114.04980426_{L}+3.73169344_{N}\right) n_{f}^{2}+1.908433871_{L} n_{f}^{3}, \\
\gamma_{\mathrm{ns}}^{(3)+}(4)= & 26818.645638_{L}+996.7898428_{N}-\left(6549.4629888_{L}+154.7093878_{N}\right) n_{f} \\
& +\left(231.33355779_{L}+7.35324122_{N}\right) n_{f}^{2}+3.884444957_{L} n_{f}^{3}, \\
\gamma_{\mathrm{ns}}^{(3)+}(6)= & 33391.136191_{L}+801.6538203_{N}-\left(8219.2929199_{L}+167.0140214_{N}\right) n_{f} \\
& +\left(299.12524225_{L}+9.35853808_{N}\right) n_{f}^{2}+5.073482406_{L} n_{f}^{3}, \\
\gamma_{\mathrm{ns}}^{(3)+}(8)= & 38122.913329_{L}+685.2274355_{N}-\left(9424.0745879_{L}+175.4883891_{N}\right) n_{f} \\
& +\left(34.752553808_{L}+10.77524037_{N}\right) n_{f}^{2}+5.937491139_{L} n_{f}^{3}, \\
\gamma_{\mathrm{ns}}^{(3)+}(10)= & 41872.765979_{L}+600.8875674_{N}-\left(10377.826722_{L}+181.7070215_{N}\right) n_{f} \\
& +\left(385.45469324_{L}+11.87446948_{N}\right) n_{f}^{2}+6.618723172_{L} n_{f}^{3}, \\
\gamma_{\mathrm{ns}}^{(3)+}(12)= & 44999.013143_{L}+533.8372124_{N}-\left(11171.437026_{L}+186.4798090_{N}\right) n_{f} \\
& +\left(416.75989357_{L}+12.77304489_{N}\right) n_{f}^{2}+7.181732766_{L} n_{f}^{3}, \\
\gamma_{\mathrm{ns}}^{(3)+}(14)= & 47688.997330_{L}+477.6377749_{N}-\left(11852.885145_{L}+190.2641681_{N}\right) n_{f} \\
& +\left(443.46765303_{L}+13.53286843_{N}\right) n_{f}^{2}+7.661731916_{L} n_{f}^{3}, \\
\gamma_{\mathrm{ns}}^{(3)+}(16)= & 50054.446557_{L}+428.9331378_{N}-\left(12450.928224_{L}+193.3399276_{N}\right) n_{f} \\
& +\left(466.78457532_{L}+14.19090917_{N}\right) n_{f}^{2}+8.080152509_{L} n_{f}^{3},
\end{aligned}
$$

and the first eight odd- $N$ values of $\gamma_{\mathrm{ns}}^{(3)-}(N)$ read

$$
\begin{aligned}
\gamma_{\mathrm{ns}}^{(3)-}(1)=0 & , \\
\gamma_{\mathrm{ns}}^{(3)-}(3)= & 22101.772707_{L}+999.3940009_{N}-\left(5359.5335080_{L}+139.7317364_{N}\right) n_{f} \\
& +\left(183.02229066_{L}+5.91723185_{N}\right) n_{f}^{2}+3.058631170_{L} n_{f}^{3}, \\
\gamma_{\mathrm{ns}}^{(3)-}(5)= & 30431.615620_{L}+841.7002595_{N}-\left(7466.3287550_{L}+160.0856189_{N}\right) n_{f} \\
& +\left(268.62851396_{L}+8.47774637_{N}\right) n_{f}^{2}+4.534727508_{L} n_{f}^{3}, \\
\gamma_{\mathrm{ns}}^{(3)-}(7)= & 35914.342449_{L}+722.2583518_{N}-\left(8861.7720221_{L}+171.1596475_{N}\right) n_{f} \\
& +\left(325.00219825_{L}+10.13425314_{N}\right) n_{f}^{2}+5.534366480_{L} n_{f}^{3}, \\
\gamma_{\mathrm{ns}}^{(3)-}(9)= & 40092.293568_{L}+632.8933507_{N}-\left(9925.1782168_{L}+178.6640945_{N}\right) n_{f} \\
& +\left(367.49639662_{L}+11.36914948_{N}\right) n_{f}^{2}+6.295934539_{L} n_{f}^{3},
\end{aligned}
$$




$$
\begin{aligned}
\gamma_{\mathrm{ns}}^{(3)-}(11)= & 43499.696829_{L}+561.6966621_{N}-\left(10791.034298_{L}+184.1995847_{N}\right) n_{f} \\
& +\left(401.78232680_{L}+12.35557815_{N}\right) n_{f}^{2}+6.912368819_{L} n_{f}^{3} \\
\gamma_{\mathrm{ns}}^{(3)-}(13)= & 46390.354670_{L}+502.2596221_{N}-\left(11524.077148_{L}+188.4837700_{N}\right) n_{f} \\
& +\left(430.59996163_{L}+13.17711657_{N}\right) n_{f}^{2}+7.430544877_{L} n_{f}^{3} \\
\gamma_{\mathrm{ns}}^{(3)-}(15)= & 48907.084426_{L}+451.0029039_{N}-\left(12160.990233_{L}+191.9086558_{N}\right) n_{f} \\
& +\left(455.49394328_{L}+13.88095204_{N}\right) n_{f}^{2}+7.877634036_{L} n_{f}^{3} .
\end{aligned}
$$

It is clear from these results, that the large- $n_{c}$ limit alone provides an excellent approximation to the individual $n_{f}^{a}$ coefficients except for the lowest values of $N$. The non-large- $n_{c}$ 'correction' amounts to $10 \%$ and $4 \%$ for the $n_{f}^{0}$ and $n_{f}^{1}$ terms, respectively, but $2 \%$ or less at $N \geq 7$ in both cases.

We have computed the first nine odd- $N$ values of the 'sea' contribution $\gamma_{\mathrm{ns}}^{(3) \mathrm{s}}(N)$ to the four-loop anomalous dimension for the overall valence distribution, and find

$$
\begin{aligned}
& \gamma_{\mathrm{ns}}^{(3) \mathrm{s}}(1)=0 \\
& \gamma_{\mathrm{ns}}^{(3) \mathrm{s}}(3)=18.9700898832 n_{f}-1.6109396433 n_{f}^{2} \\
& \gamma_{\mathrm{ns}}^{(3) \mathrm{s}}(5)=9.1402406178 n_{f}+0.1525610933 n_{f}^{2} \\
& \gamma_{\mathrm{ns}}^{(3) \mathrm{s}}(7)=4.0470106556 n_{f}+0.3095914493 n_{f}^{2} \\
& \gamma_{\mathrm{ns}}^{(3) \mathrm{s}}(9)=1.9658456985 n_{f}+0.2887522942 n_{f}^{2} \\
& \gamma_{\mathrm{ns}}^{(3) \mathrm{s}}(11)=1.0102117327 n_{f}+0.2474854100 n_{f}^{2} \\
& \gamma_{\mathrm{ns}}^{(3) \mathrm{s}}(13)=0.5255291656 n_{f}+0.2099342070 n_{f}^{2} \\
& \gamma_{\mathrm{ns}}^{(3) \mathrm{s}}(15)=0.2612077170 n_{f}+0.1791623892 n_{f}^{2} \\
& \gamma_{\mathrm{ns}}^{(3) \mathrm{s}}(17)=0.1094470531 n_{f}+0.1544243611 n_{f}^{2}
\end{aligned}
$$

We now turn to the analytic all- $N$ expressions for the $n_{f}^{0}$ and $n_{f}^{1}$ parts of the fourloop non-singlet anomalous dimensions $\gamma_{\mathrm{ns}}^{(3) \pm}$ in the large- $n_{c}$ limit. The complete lowerorder contributions can be found, in a different notation but the same normalization, in eqs. (3.4) - (3.8) of ref. [14]. The anomalous dimensions can be expressed in terms of the denominators $D_{a}^{k}$ in eq. (2.14) and harmonic sums [37,38] at argument $N$, which are recursively defined by

$$
\begin{aligned}
S_{ \pm m}(N) & =\sum_{n=1}^{N}( \pm 1)^{n} n^{-m} \\
S_{ \pm m_{1}, m_{2}, \ldots, m_{d}}(N) & =\sum_{n=1}^{N}( \pm 1)^{n} n^{-m_{1}} S_{m_{2}, \ldots, m_{d}}(n) .
\end{aligned}
$$

The weight $w$ of the harmonic sums is defined by the sum of the absolute values of the indices $m_{d}$. Sums up to $w=2 n-1$ occur in the $n$-loop anomalous dimensions. The argument $N$ of the sums is suppressed for brevity below, and we use the shorthand $\eta=$ $1 /(N(N+1))=D_{0} D_{1}$. 
The identical large- $n_{c}$ parts of the functions $\gamma_{\mathrm{ns}}^{(3)+}(N)$ and $\gamma_{\mathrm{ns}}^{(3)-}(N)$ are given by

$$
\gamma_{\mathrm{ns}, \mathrm{L}}^{(3)}(N)=C_{F}\left(n_{c}^{3} \gamma_{\mathrm{L}, 0}^{(3)}(N)+n_{c}^{2} n_{f} \gamma_{\mathrm{L}, 1}^{(3)}(N)+n_{c} n_{f}^{2} \gamma_{\mathrm{L}, 2}^{(3)}(N)+n_{f}^{3} \gamma_{\mathrm{L}, 3}^{(3)}(N)\right)
$$

where the $n_{f}^{2}$ and $n_{f}^{3}$ contributions to eq. (3.5) have been given in eqs. (3.1) and (3.6) of ref. [32]; the latter has first been derived in ref. [102]. Our new results are

$$
\begin{aligned}
& \gamma_{\mathrm{L}, 0}^{(3)}(N)= \\
& 16\left(1379569 / 82944-7453 / 24 D_{1}^{2}-102641 / 648 D_{1}^{3}-9883 / 864 D_{1}^{4}-209 / 12 D_{1}^{5}\right. \\
& -95 / 12 D_{1}^{6}-5 / 4 D_{1}^{7}+534767 / 5184 \eta-231341 / 2592 \eta^{2}-15469 / 1296 \eta^{3}-12997 / 864 \eta^{4} \\
& -83 / 12 \eta^{5}-25 / 24 \eta^{6}-5 / 8 \eta^{7}-55 / 4 \zeta_{5}+40 \zeta_{5} \eta-25 \zeta_{5} \eta^{2}-1517 / 144 \zeta_{3}+839 / 108 \zeta_{3} \eta \\
& +13 / 24 \zeta_{3} \eta^{2}-2 / 3 \zeta_{3} \eta^{3}-1 / 2 \zeta_{3} \eta^{4}+42139 / 648 S_{1}-130795 / 648 S_{1} D_{1}^{2}-298 / 9 S_{1} D_{1}^{3} \\
& -995 / 24 S_{1} D_{1}^{4}-92 / 3 S_{1} D_{1}^{5}-15 / 2 S_{1} D_{1}^{6}+278627 / 1296 S_{1} \eta+19757 / 2592 S_{1} \eta^{2} \\
& +3625 / 48 S_{1} \eta^{3}+1789 / 72 S_{1} \eta^{4}+9 S_{1} \eta^{5}+7 / 2 S_{1} \eta^{6}+422 / 27 S_{1} \zeta_{3}-62 / 3 S_{1} \zeta_{3} \eta \\
& -7 / 3 S_{1} \zeta_{3} \eta^{2}+10 S_{1} \zeta_{3} \eta^{3}-24211 / 432 S_{2}+23153 / 216 S_{2} D_{1}^{2}+143 / 3 S_{2} D_{1}^{3}+16 S_{2} D_{1}^{4} \\
& +10 S_{2} D_{1}^{5}-18725 / 1296 S_{2} \eta+23689 / 432 S_{2} \eta^{2}+3187 / 144 S_{2} \eta^{3}+229 / 12 S_{2} \eta^{4}+29 / 4 S_{2} \eta^{5} \\
& -20 S_{2} \zeta_{3} \eta+8 S_{2} \zeta_{3} \eta^{2}+71591 / 864 S_{3}-5099 / 72 S_{3} D_{1}^{2}-5 / 3 S_{3} D_{1}^{3}-2 S_{3} D_{1}^{4}+4373 / 108 S_{3} \eta \\
& +1051 / 72 S_{3} \eta^{2}+40 / 3 S_{3} \eta^{3}+27 / 4 S_{3} \eta^{4}-13 / 3 S_{3} \zeta_{3}+2 S_{3} \zeta_{3} \eta-55291 / 864 S_{4} \\
& +155 / 6 S_{4} D_{1}^{2}+7 S_{4} D_{1}^{3}+3233 / 144 S_{4} \eta+103 / 12 S_{4} \eta^{2}+11 / 2 S_{4} \eta^{3}+4 S_{4} \zeta_{3}+227 / 4 S_{5} \\
& -12 S_{5} D_{1}^{2}+1 / 3 S_{5} \eta+7 S_{5} \eta^{2}-65 / 3 S_{6}+10 S_{6} \eta+10 S_{7}-56 / 3 S_{1,1} D_{1}^{2}-71 / 3 S_{1,1} D_{1}^{3} \\
& -35 S_{1,1} D_{1}^{4}-20 S_{1,1} D_{1}^{5}+23 / 12 S_{1,1} \eta-587 / 8 S_{1,1} \eta^{2}-115 / 4 S_{1,1} \eta^{3}-109 / 3 S_{1,1} \eta^{4} \\
& -11 S_{1,1} \eta^{5}+40 S_{1,1} \zeta_{3} \eta-16 S_{1,1} \zeta_{3} \eta^{2}-5552 / 81 S_{1,2}+184 / 3 S_{1,2} D_{1}^{2}-2 S_{1,2} D_{1}^{3}+6 S_{1,2} D_{1}^{4} \\
& -383 / 6 S_{1,2} \eta-241 / 12 S_{1,2} \eta^{2}-137 / 6 S_{1,2} \eta^{3}-17 / 2 S_{1,2} \eta^{4}+890 / 27 S_{1,3}-22 / 3 S_{1,3} D_{1}^{2} \\
& +53 / 3 S_{1,3} \eta-79 / 6 S_{1,3} \eta^{2}-11 S_{1,3} \eta^{3}-4 S_{1,3} \zeta_{3}-4745 / 72 S_{1,4}+14 S_{1,4} D_{1}^{2}-46 / 3 S_{1,4} \eta \\
& -S_{1,4} \eta^{2}+70 / 3 S_{1,5}-4 S_{1,5} \eta-20 S_{1,6}-5552 / 81 S_{2,1}+184 / 3 S_{2,1} D_{1}^{2}-2 S_{2,1} D_{1}^{3}+6 S_{2,1} D_{1}^{4} \\
& -383 / 6 S_{2,1} \eta-241 / 12 S_{2,1} \eta^{2}-137 / 6 S_{2,1} \eta^{3}-17 / 2 S_{2,1} \eta^{4}+9029 / 216 S_{2,2}-19 S_{2,2} D_{1}^{2} \\
& -6 S_{2,2} D_{1}^{3}-38 / 3 S_{2,2} \eta-18 S_{2,2} \eta^{2}-9 S_{2,2} \eta^{3}-3635 / 72 S_{2,3}+12 S_{2,3} D_{1}^{2}+5 / 3 S_{2,3} \eta \\
& -10 S_{2,3} \eta^{2}+31 / 2 S_{2,4}+3 S_{2,4} \eta-16 S_{2,5}+925 / 18 S_{3,1}-76 / 3 S_{3,1} D_{1}^{2}-8 S_{3,1} D_{1}^{3} \\
& -1675 / 36 S_{3,1} \eta-21 S_{3,1} \eta^{2}-4 S_{3,1} \eta^{3}-4 S_{3,1} \zeta_{3}-5581 / 72 S_{3,2}+22 S_{3,2} D_{1}^{2}-53 / 3 S_{3,2} \eta \\
& -16 S_{3,2} \eta^{2}+143 / 6 S_{3,3}-11 S_{3,3} \eta-14 S_{3,4}-6899 / 72 S_{4,1}+24 S_{4,1} D_{1}^{2}-74 / 3 S_{4,1} \eta \\
& -11 S_{4,1} \eta^{2}+57 / 2 S_{4,2}-25 S_{4,2} \eta-26 S_{4,3}+63 / 2 S_{5,1}-23 S_{5,1} \eta-36 S_{5,2}-28 S_{6,1} \\
& -12 S_{1,1,1} D_{1}^{4}+12 S_{1,1,1} \eta^{2}+24 S_{1,1,1} \eta^{3}+6 S_{1,1,1} \eta^{4}+18 S_{1,1,2} \eta^{2}+6 S_{1,1,2} \eta^{3}-20 S_{1,1,3} \eta \\
& +8 S_{1,1,3} \eta^{2}+20 / 3 S_{1,1,4}-20 S_{1,1,4} \eta+8 S_{1,1,5}+18 S_{1,2,1} \eta^{2}+6 S_{1,2,1} \eta^{3}+134 / 3 S_{1,2,2} \\
& -12 S_{1,2,2} D_{1}^{2}+12 S_{1,2,2} \eta+6 S_{1,2,2} \eta^{2}-22 / 3 S_{1,2,3}-6 S_{1,2,4}+1447 / 18 S_{1,3,1}-16 S_{1,3,1} D_{1}^{2} \\
& +104 / 3 S_{1,3,1} \eta-6 S_{1,3,1} \eta^{2}-38 / 3 S_{1,3,2}+16 S_{1,3,2} \eta+22 S_{1,3,3}-56 / 3 S_{1,4,1}+12 S_{1,4,1} \eta \\
& +50 S_{1,4,2}+46 S_{1,5,1}+18 S_{2,1,1} \eta^{2}+6 S_{2,1,1} \eta^{3}+134 / 3 S_{2,1,2}-12 S_{2,1,2} D_{1}^{2}+12 S_{2,1,2} \eta \\
& +6 S_{2,1,2} \eta^{2}-22 / 3 S_{2,1,3}-6 S_{2,1,4}+134 / 3 S_{2,2,1}-12 S_{2,2,1} D_{1}^{2}+12 S_{2,2,1} \eta+6 S_{2,2,1} \eta^{2}
\end{aligned}
$$




$$
\begin{aligned}
& -13 S_{2,2,2}+6 S_{2,2,2} \eta+12 S_{2,2,3}-44 / 3 S_{2,3,1}+38 S_{2,3,2}+36 S_{2,4,1}+307 / 6 S_{3,1,1}-20 S_{3,1,1} D_{1}^{2} \\
& +86 / 3 S_{3,1,1} \eta+16 S_{3,1,1} \eta^{2}-43 / 3 S_{3,1,2}+10 S_{3,1,2} \eta+14 S_{3,1,3}-43 / 3 S_{3,2,1}+10 S_{3,2,1} \eta \\
& +24 S_{3,2,2}+22 S_{3,3,1}-37 / 3 S_{4,1,1}+26 S_{4,1,1} \eta+28 S_{4,1,2}+28 S_{4,2,1}+44 S_{5,1,1}+40 S_{1,1,1,4} \\
& -16 / 3 S_{1,1,3,1}+16 S_{1,1,3,1} \eta-32 S_{1,1,3,2}-24 S_{1,1,4,1}-12 S_{1,2,2,2}-28 / 3 S_{1,3,1,1}-16 S_{1,3,1,1} \eta \\
& -20 S_{1,3,1,2}-20 S_{1,3,2,1}-52 S_{1,4,1,1}-12 S_{2,1,2,2}-12 S_{2,2,1,2}-12 S_{2,2,2,1}-36 S_{2,3,1,1} \\
& -12 S_{3,1,1,2}-12 S_{3,1,2,1}-12 S_{3,2,1,1}-12 S_{4,1,1,1}-32 S_{1,1,1,3,1}+32 S_{1,1,3,1,1}
\end{aligned}
$$

and

$$
\begin{aligned}
& \gamma_{\mathrm{L}, 1}^{(3)}(N)= \\
& 16\left(-353 / 48+119917 / 864 D_{1}^{2}+15689 / 324 D_{1}^{3}+433 / 72 D_{1}^{4}+19 / 3 D_{1}^{5}+5 / 3 D_{1}^{6}\right. \\
& -112979 / 2592 \eta+13405 / 648 \eta^{2}-8045 / 1296 \eta^{3}-61 / 18 \eta^{4}-1 / 2 \eta^{5}-5 / 6 \eta^{6}-5 \zeta_{5} \\
& -15 \zeta_{5} \eta+10 \zeta_{5} \eta^{2}-33 / 8 \zeta_{4}-11 / 4 \zeta_{4} \eta+235 / 16 \zeta_{3}+8 / 3 \zeta_{3} D_{1}^{2}+2 \zeta_{3} D_{1}^{3}+83 / 8 \zeta_{3} \eta \\
& +3 / 2 \zeta_{3} \eta^{2}+2 / 3 \zeta_{3} \eta^{3}-39883 / 1296 S_{1}+19009 / 324 S_{1} D_{1}^{2}+77 / 9 S_{1} D_{1}^{3}+79 / 6 S_{1} D_{1}^{4} \\
& +20 / 3 S_{1} D_{1}^{5}-19927 / 324 S_{1} \eta+1453 / 81 S_{1} \eta^{2}-7 / 24 S_{1} \eta^{3}+38 / 9 S_{1} \eta^{4}+3 S_{1} \eta^{5} \\
& +10 S_{1} \zeta_{5}+11 / 2 S_{1} \zeta_{4}-317 / 12 S_{1} \zeta_{3}+4 S_{1} \zeta_{3} D_{1}^{2}+8 / 3 S_{1} \zeta_{3} \eta-8 / 3 S_{1} \zeta_{3} \eta^{2}-4 S_{1} \zeta_{3} \eta^{3} \\
& +85175 / 2592 S_{2}-1873 / 54 S_{2} D_{1}^{2}-20 / 3 S_{2} D_{1}^{3}-4 S_{2} D_{1}^{4}+4943 / 648 S_{2} \eta+95 / 216 S_{2} \eta^{2} \\
& +229 / 36 S_{2} \eta^{3}+25 / 6 S_{2} \eta^{4}+2 / 3 S_{2} \zeta_{3}+2 S_{2} \zeta_{3} \eta-4 S_{2} \zeta_{3} \eta^{2}-22247 / 648 S_{3}+241 / 18 S_{3} D_{1}^{2} \\
& +2 / 3 S_{3} D_{1}^{3}-113 / 54 S_{3} \eta+37 / 18 S_{3} \eta^{2}+19 / 6 S_{3} \eta^{3}-8 / 3 S_{3} \zeta_{3}+725 / 24 S_{4}-16 / 3 S_{4} D_{1}^{2} \\
& -73 / 36 S_{4} \eta+5 / 3 S_{4} \eta^{2}-46 / 3 S_{5}+8 / 3 S_{5} \eta+20 / 3 S_{6}+8 / 3 S_{1,1} D_{1}^{2}+8 / 3 S_{1,1} D_{1}^{3}+8 S_{1,1} D_{1}^{4} \\
& +4 / 3 S_{1,1} \eta+9 / 4 S_{1,1} \eta^{2}-12 S_{1,1} \eta^{3}-14 / 3 S_{1,1} \eta^{4}-8 S_{1,1} \zeta_{3} \eta+8 S_{1,1} \zeta_{3} \eta^{2}+6673 / 324 S_{1,2} \\
& -28 / 3 S_{1,2} D_{1}^{2}+28 / 3 S_{1,2} \eta-8 / 3 S_{1,2} \eta^{2}-8 / 3 S_{1,2} \eta^{3}+4 S_{1,2} \zeta_{3}-605 / 54 S_{1,3}+4 / 3 S_{1,3} D_{1}^{2} \\
& -14 / 3 S_{1,3} \eta-1 / 3 S_{1,3} \eta^{2}+2 S_{1,3} \eta^{3}+181 / 18 S_{1,4}+10 / 3 S_{1,4} \eta-16 / 3 S_{1,5}+6673 / 324 S_{2,1} \\
& -28 / 3 S_{2,1} D_{1}^{2}+28 / 3 S_{2,1} \eta-8 / 3 S_{2,1} \eta^{2}-8 / 3 S_{2,1} \eta^{3}+4 S_{2,1} \zeta_{3}-1021 / 54 S_{2,2}+4 S_{2,2} D_{1}^{2} \\
& +2 / 3 S_{2,2} \eta-2 S_{2,2} \eta^{2}+181 / 18 S_{2,3}-8 / 3 S_{2,3} \eta+2 S_{2,3} \eta^{2}-2 S_{2,4}-479 / 18 S_{3,1} \\
& +16 / 3 S_{3,1} D_{1}^{2}+59 / 9 S_{3,1} \eta-2 S_{3,1} \eta^{2}-2 S_{3,1} \eta^{3}+275 / 18 S_{3,2}-10 / 3 S_{3,2} \eta-22 / 3 S_{3,3} \\
& +343 / 18 S_{4,1}-4 / 3 S_{4,1} \eta-2 S_{4,1} \eta^{2}-12 S_{4,2}-12 S_{5,1}+4 S_{1,1,3} \eta-4 S_{1,1,3} \eta^{2}-20 / 3 S_{1,1,4} \\
& -20 / 3 S_{1,2,2}+4 / 3 S_{1,2,3}-94 / 9 S_{1,3,1}-20 / 3 S_{1,3,1} \eta+4 S_{1,3,1} \eta^{2}+20 / 3 S_{1,3,2}+20 / 3 S_{1,4,1} \\
& -20 / 3 S_{2,1,2}+4 / 3 S_{2,1,3}-20 / 3 S_{2,2,1}+4 S_{2,2,2}+8 / 3 S_{2,3,1}-20 / 3 S_{3,1,1}+4 / 3 S_{3,1,1} \eta \\
& \left.+16 / 3 S_{3,1,2}+16 / 3 S_{3,2,1}+28 / 3 S_{4,1,1}+16 / 3 S_{1,1,3,1}-8 / 3 S_{1,3,1,1}\right) \text {. }
\end{aligned}
$$

The large- $N$ limit of eq. (3.5) is of the form (2.17) with the large- $n_{c}$ cusp anomalous dimension

$$
\begin{aligned}
A_{L, 4}= & C_{F} n_{c}^{3}\left(\frac{84278}{81}-\frac{88832}{81} \zeta_{2}+\frac{20992}{27} \zeta_{3}+1804 \zeta_{4}-\frac{352}{3} \zeta_{2} \zeta_{3}-352 \zeta_{5}-32 \zeta_{3}^{2}-876 \zeta_{6}\right) \\
& -C_{F} n_{c}^{2} n_{f}\left(\frac{39883}{81}-\frac{26692}{81} \zeta_{2}+\frac{16252}{27} \zeta_{3}+\frac{440}{3} \zeta_{4}-\frac{256}{3} \zeta_{2} \zeta_{3}-224 \zeta_{5}\right)
\end{aligned}
$$




$$
+C_{F} n_{c} n_{f}^{2}\left(\frac{2119}{81}-\frac{608}{81} \zeta_{2}+\frac{1280}{27} \zeta_{3}-\frac{64}{3} \zeta_{4}\right)-C_{F} n_{f}^{3}\left(\frac{32}{81}-\frac{64}{27} \zeta_{3}\right) .
$$

Our result for the (complete) $n_{f}^{2}$ part was first presented at Loops \& Legs 2016, see ref. [31], the rest in a Zurich seminar by one of us [103]. Eq. (3.8) agrees with results of refs. [46, 47], where this quantity was obtained by computing the photon-quark form factor in the large$n_{c}$ limit. The lower-order coefficients can be found in eq. (3.11) of ref. [14].

The one- to three-loop coefficients $B_{1,2,3}$ in eq. (4.9) can be found, as coefficients of $\delta(1-x)$, in eqs. (4.5), (4.6) and (4.9) of ref. [14]. The four-loop coefficient in the large- $n_{c}$ limit reads

$$
\begin{aligned}
B_{L, 4}= & C_{F} n_{c}^{3}\left(-\frac{1379569}{5184}+\frac{24211}{27} \zeta_{2}-\frac{9803}{162} \zeta_{3}-\frac{9382}{9} \zeta_{4}+\frac{838}{9} \zeta_{2} \zeta_{3}+1002 \zeta_{5}\right. \\
& \left.+\frac{16}{3} \zeta_{3}^{2}+135 \zeta_{6}-80 \zeta_{2} \zeta_{5}+32 \zeta_{3} \zeta_{4}-560 \zeta_{7}\right) \\
& +C_{F} n_{c}^{2} n_{f}\left(\frac{353}{3}-\frac{85175}{162} \zeta_{2}-\frac{137}{9} \zeta_{3}+\frac{16186}{27} \zeta_{4}-\frac{584}{9} \zeta_{2} \zeta_{3}-\frac{248}{3} \zeta_{5}-\frac{16}{3} \zeta_{3}^{2}-144 \zeta_{6}\right) \\
& -C_{F} n_{c} n_{f}^{2}\left(\frac{127}{18}-\frac{5036}{81} \zeta_{2}+\frac{932}{27} \zeta_{3}+\frac{1292}{27} \zeta_{4}-\frac{160}{9} \zeta_{2} \zeta_{3}-\frac{32}{3} \zeta_{5}\right) \\
& -C_{F} n_{f}^{3}\left(\frac{131}{81}-\frac{32}{81} \zeta_{2}-\frac{304}{81} \zeta_{3}+\frac{32}{27} \zeta_{4}\right) .
\end{aligned}
$$

The coefficients $B_{n}$ contain collinear contributions to the evolution kernels. With the help of the QCD corrections to the quark form factor in dimensional regularization, one can extract from them the universal eikonal anomalous dimension. The latter governs the subleading infrared poles in gauge-theory amplitudes and captures contributions from large-angle soft gluons [100, 104, 105].

As mentioned above, the coefficients $C_{n}$ and $\widetilde{D}_{n}$ in eq. (4.9) do not provide new information, but are functions of lower-order quantities. They are given by

$$
C\left(a_{\mathrm{s}}\right)=\left(A\left(a_{\mathrm{s}}\right)\right)^{2}, \quad \widetilde{D}\left(a_{\mathrm{s}}\right)=A\left(a_{\mathrm{s}}\right) \cdot\left(B\left(a_{\mathrm{s}}\right)-\beta\left(a_{\mathrm{s}}\right) / a_{\mathrm{s}}\right),
$$

cf. ref. [65], which leads to the four-loop relations

$$
C_{4}=A_{2}^{2}+2 A_{1} A_{3}, \quad \widetilde{D}_{4}=\sum_{k=1}^{3} A_{k} \cdot\left(B_{4-k}-\beta_{3-k}\right) .
$$

Using the results (3.8) and (3.9), it is actually now possible to predict $C_{5}$ and $\widetilde{D}_{5}$ for large $n_{c}$.

The new functions (3.6) and (3.7) are shown in figures 2 and 3, respectively, together with their large- $N$ approximation (2.17) with the coefficients given above. In the right panels, the results are divided by $\ln N$, so for $N \rightarrow \infty$ the curves tend to constants given by the respective terms in the four-loop cusp anomalous dimension (3.8).

The approach to this asymptotic behaviour is very slow: the $n_{f}^{0}$ contribution in figure 2 is 0.856 of its asymptotic result at $N=30$, yet it deviates by less than $10 \%$ only from an $N$-value above $N=500$. The corresponding numbers for the $n_{f}^{1}$ part in figure 3 are 

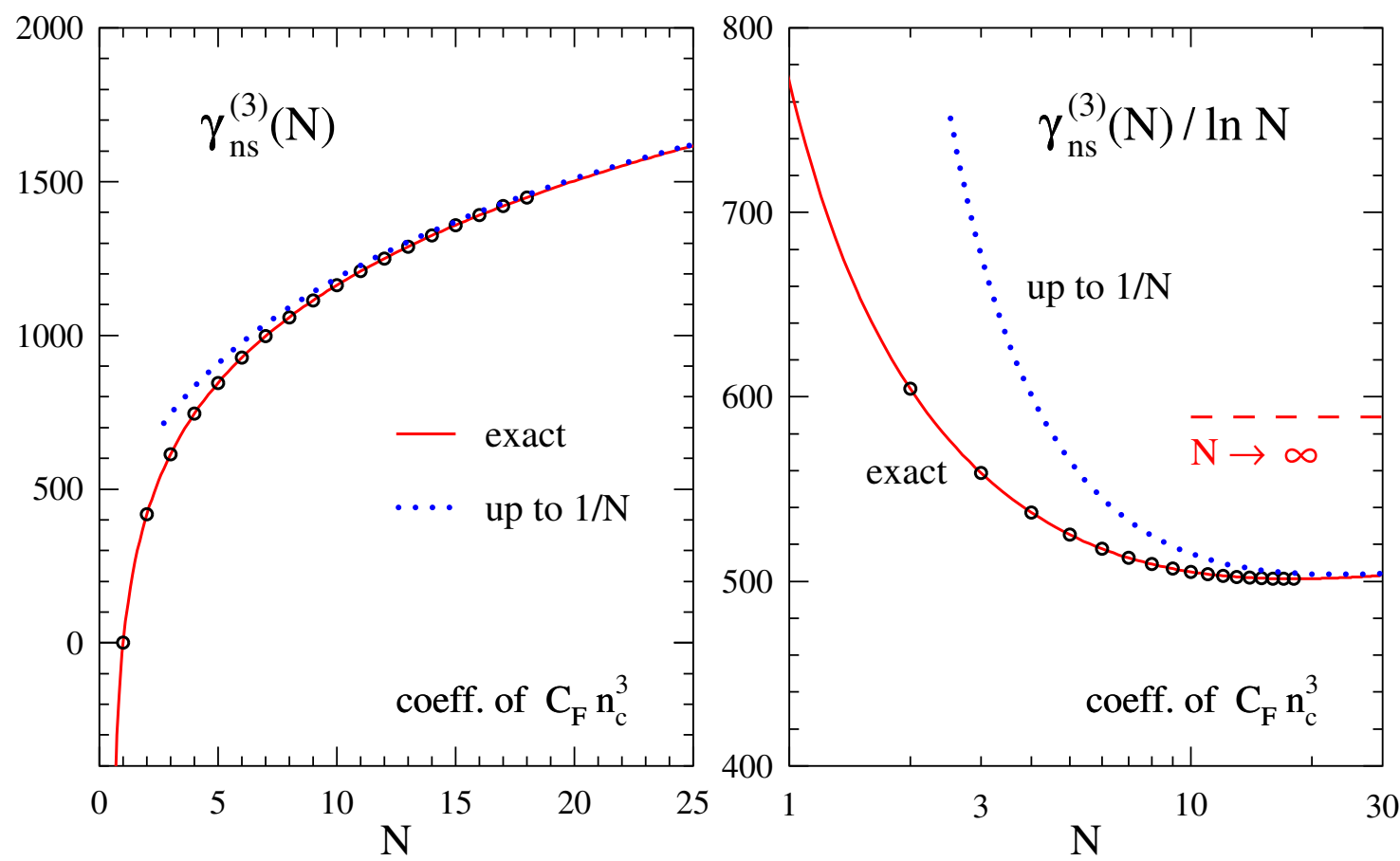

Figure 2. The $n_{f}^{0}$ part (3.6) of the anomalous dimensions $\gamma_{\mathrm{ns}}^{(n) \pm}(N)$ in the large- $n_{c}$ limit, compared with its large- $N$ expansion with all terms included in eq. (2.17) and, in the right panel, its asymptotic behaviour for $N \rightarrow \infty$. The exact curve has been computed by via the $x$-space counterpart of eq. (3.6), see section 4 .

0.873 at $N=30$ and $N \simeq 185$ for a deviation by less than $10 \%$. It might be interesting to note, on the other hand, that the corresponding $n_{f}^{a}$ coefficient of $A_{n}$, here and in all lower-order cases (in full QCD), falls in the interval spanned by the corresponding results for $\gamma_{\mathrm{ns}}^{(n)}(2) / \ln 2$ and $\gamma_{\mathrm{ns}}^{(n)}(4) / \ln 4$.

The results (3.1) for $\gamma_{\mathrm{ns}}^{(3)+}$ (closed circles) and (3.2) for $\gamma_{\mathrm{ns}}^{(3)-}$ (open circles) are shown for the physically relevant values of $n_{f}$ in figure 4 , together with the all- $N$ results in the large- $n_{c}$ limit. As at the previous orders in $\alpha_{\mathrm{s}}$, there are cancellations between the $n_{f^{-}}$ independent and the $n_{f}$-dependent contributions, which are particularly pronounced here at $n_{f}=5$. For this number of light flavours, which is relevant for high-energy processes at the LHC, the large- $n_{c}$ result do not describe the (small) fourth-order QCD contributions to the non-singlet evolution equations at the phenomenologically most relevant moments $N$ and momentum fractions $x$. We therefore need to convert the calculated moments to practically usable constraints on the four-loop splitting functions $P_{\mathrm{ns}}^{(3)}(x)$.

\section{Results in $x$-space}

The four-loop non-singlet splitting functions $P_{\mathrm{ns}}^{(3)}(x)$ are derived from the all- $N$ results for the corresponding anomalous dimensions by an inverse Mellin transformation that expresses these functions in terms of harmonic polylogarithms (HPLs). This transformation can be 

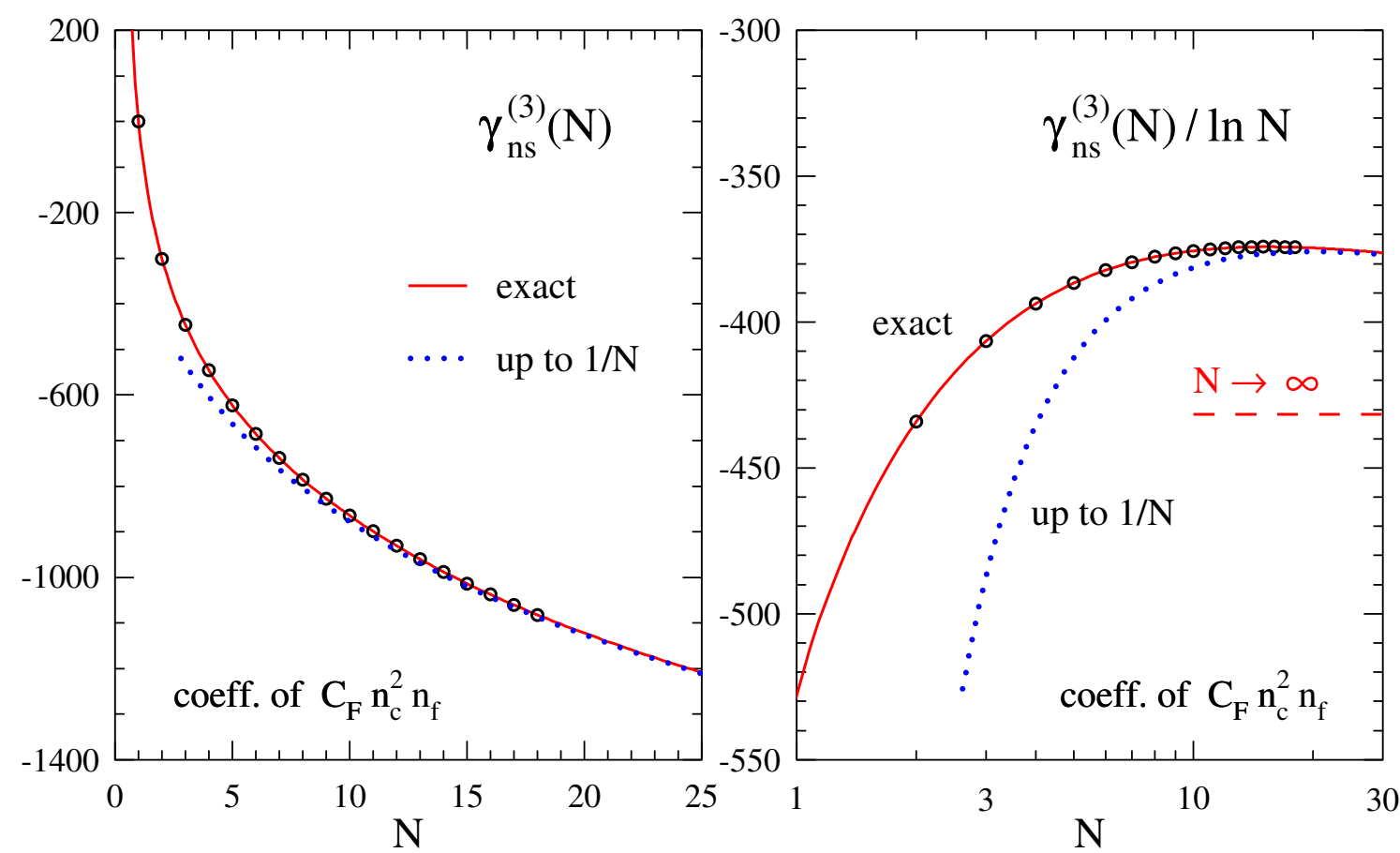

Figure 3. As figure 2, but for the $n_{f}^{1}$ contribution (3.7). The value of $\gamma_{\mathrm{ns}}^{(n) \pm}(N) / \ln N$ in the limit $N \rightarrow \infty$ is given by the corresponding coefficient of $A_{L, 4}$ in the second line of eq. (3.8).
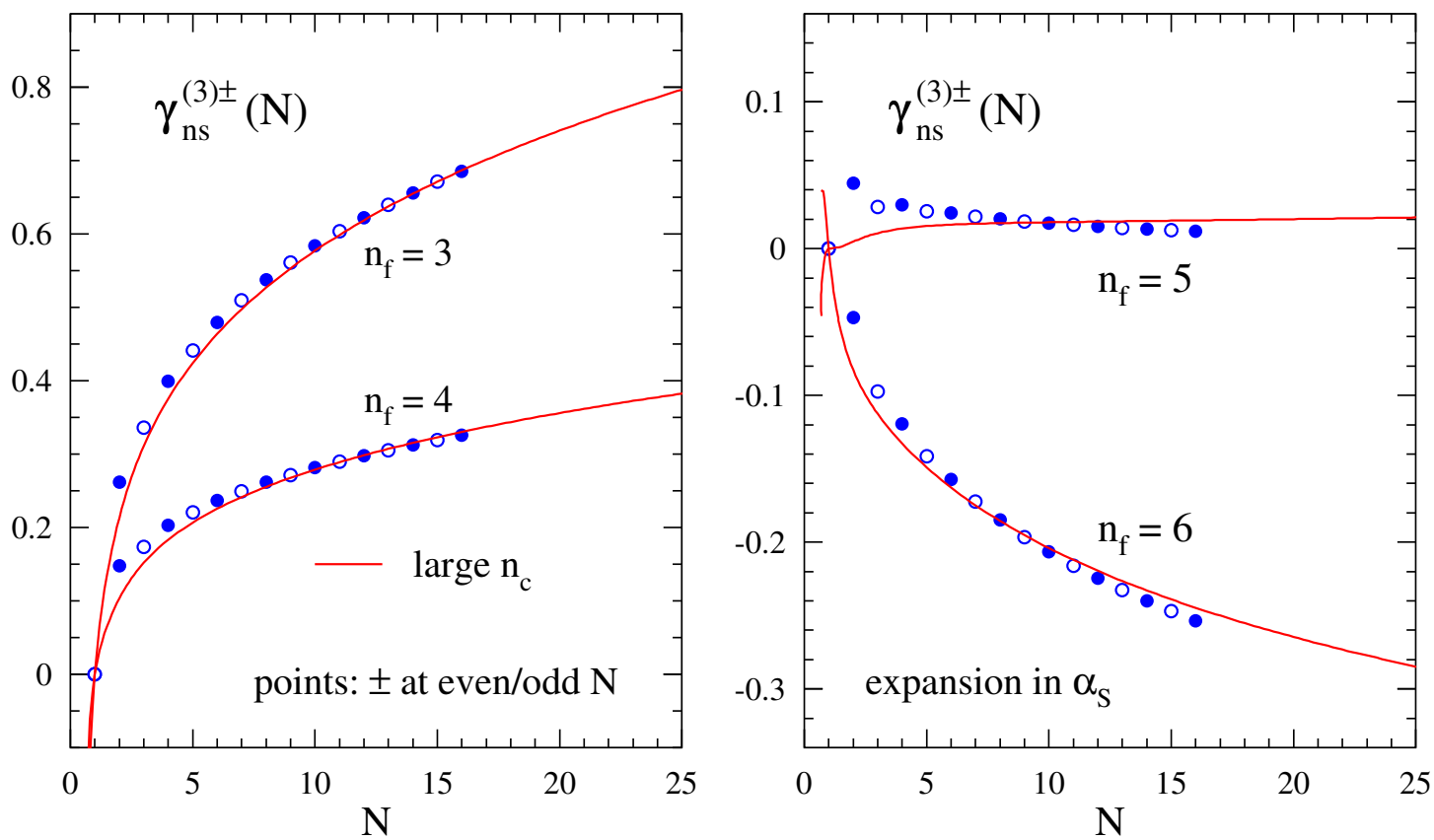

Figure 4. Our even- $N$ results for $\gamma_{\text {ns }}^{(n)+}(N)$ and odd- $N$ values for $\gamma_{\mathrm{ns}}^{(n)-}(N)$ at $n_{f}=3, \ldots 6$, compared with their common large- $n_{c}$ limit now known at all $N$. The results have been converted to an expansion in $\alpha_{\mathrm{s}}$. 
performed by an algebraic procedure $[87,88]$ based on the fact that harmonic sums occur as coefficients of the Taylor expansion of HPLs.

For the convenience of the reader, we recall their basic definitions [87]. The lowestweight $(w=1)$ functions $H_{m}(x)$ are given by

$$
H_{0}(x)=\ln x, \quad H_{ \pm 1}(x)=\mp \ln (1 \mp x) .
$$

The higher-weight $(w \geq 2)$ functions are recursively defined as

$$
H_{m_{1}, \ldots, m_{w}}(x)=\left\{\begin{array}{cl}
\frac{1}{w !} \ln ^{w} x, & \text { if } m_{1}, \ldots, m_{w}=0, \ldots, 0 \\
\int_{0}^{x} d z f_{m_{1}}(z) H_{m_{2}, \ldots, m_{w}}(z), & \text { otherwise }
\end{array}\right.
$$

with

$$
f_{0}(x)=x^{-1}, \quad f_{ \pm 1}(x)=(1 \mp x)^{-1} .
$$

For chains of indices 'zero' we employ the abbreviated notation

$$
H_{m}^{H_{0, \ldots, 0}}, \pm 1, \underbrace{0, \ldots, 0}_{n}, \pm 1, \ldots(x)=H_{ \pm(m+1), \pm(n+1), \ldots}(x) \text {. }
$$

The argument $x$ will be suppressed in all results below, and we express the terms with $(1 \pm x)^{-1}$ in terms of the $x$-dependence of the leading-order splitting function $P_{\mathrm{qq}}^{(0)}$,

$$
p_{\mathrm{qq}}(x)=2(1-x)^{-1}-1-x .
$$

In this notation, the common large- $n_{c}$ limit of the functions $P_{\mathrm{ns}}^{(3)+}(x)$ and $P_{\mathrm{ns}}^{(3)-}(x)$ is given by

$$
P_{\mathrm{ns}, \mathrm{L}}^{(3)}(x)=C_{F}\left(n_{c}^{3} P_{\mathrm{L}, 0}^{(3)}(x)+n_{c}^{2} n_{f} P_{\mathrm{L}, 1}^{(3)}(x)+n_{c} n_{f}^{2} P_{\mathrm{L}, 2}^{(3)}(x)+n_{f}^{3} P_{\mathrm{L}, 3}^{(3)}(x)\right)
$$

with the new results

$$
\begin{aligned}
& P_{\mathrm{L}, 0}^{(3)}(x)= \\
& p_{\mathrm{qq}}(x)\left(\frac{42139}{81}-160 \zeta_{6}-204 \zeta_{5}+\frac{7666}{9} \zeta_{4}+\frac{10334}{27} \zeta_{3}+16 \zeta_{3}^{2}-\frac{44416}{81} \zeta_{2}-72 \zeta_{2} \zeta_{3}\right. \\
& +\frac{71591}{108} \mathrm{H}_{0,0}+504 \mathrm{H}_{0,0} \zeta_{4}+\frac{212}{3} \mathrm{H}_{0,0} \zeta_{3}-\frac{6899}{9} \mathrm{H}_{0,0} \zeta_{2}+\frac{44416}{81} \mathrm{H}_{1,0}+680 \mathrm{H}_{1,0} \zeta_{4} \\
& +128 \mathrm{H}_{1,0} \zeta_{3}-\frac{5788}{9} \mathrm{H}_{1,0} \zeta_{2}+512 \mathrm{H}_{1,1} \zeta_{4}+\frac{128}{3} \mathrm{H}_{1,1} \zeta_{3}+192 \mathrm{H}_{1,2} \zeta_{3}+\frac{5788}{9} \mathrm{H}_{1,3} \\
& -160 \mathrm{H}_{1,3} \zeta_{2}+\frac{448}{3} \mathrm{H}_{1,4}+368 \mathrm{H}_{1,5}+\frac{9029}{27} \mathrm{H}_{2,0}+32 \mathrm{H}_{2,0} \zeta_{3}-\frac{352}{3} \mathrm{H}_{2,0} \zeta_{2}+192 \mathrm{H}_{2,1} \zeta_{3} \\
& +\frac{1072}{3} \mathrm{H}_{2,2}-96 \mathrm{H}_{2,2} \zeta_{2}+\frac{352}{3} \mathrm{H}_{2,3}+288 \mathrm{H}_{2,4}+\frac{5581}{9} \mathrm{H}_{3,0}-176 \mathrm{H}_{3,0} \zeta_{2}+\frac{1228}{3} \mathrm{H}_{3,1} \\
& -96 \mathrm{H}_{3,1} \zeta_{2}+\frac{344}{3} \mathrm{H}_{3,2}+176 \mathrm{H}_{3,3}+228 \mathrm{H}_{4,0}+\frac{296}{3} \mathrm{H}_{4,1}+224 \mathrm{H}_{4,2}+288 \mathrm{H}_{5,0}+352 \mathrm{H}_{5,1} \\
& +\frac{55291}{108} \mathrm{H}_{0,0,0}-32 \mathrm{H}_{0,0,0} \zeta_{3}-252 \mathrm{H}_{0,0,0} \zeta_{2}+\frac{7120}{27} \mathrm{H}_{1,0,0}-16 \mathrm{H}_{1,0,0} \zeta_{3}-\frac{448}{3} \mathrm{H}_{1,0,0} \zeta_{2}
\end{aligned}
$$




$$
\begin{aligned}
& +64 \mathrm{H}_{1,1,0} \zeta_{3}+\frac{128}{3} \mathrm{H}_{1,1,0} \zeta_{2}+256 \mathrm{H}_{1,1,1} \zeta_{3}-\frac{128}{3} \mathrm{H}_{1,1,3}+192 \mathrm{H}_{1,1,4}+\frac{1072}{3} \mathrm{H}_{1,2,0} \\
& +\frac{304}{3} \mathrm{H}_{1,3,0}-\frac{224}{3} \mathrm{H}_{1,3,1}+160 \mathrm{H}_{1,3,2}+400 \mathrm{H}_{1,4,0}+416 \mathrm{H}_{1,4,1}+\frac{3635}{9} \mathrm{H}_{2,0,0} \\
& -288 \mathrm{H}_{2,0,0} \zeta_{2}+\frac{1072}{3} \mathrm{H}_{2,1,0}+104 \mathrm{H}_{2,2,0}+96 \mathrm{H}_{2,2,2}+304 \mathrm{H}_{2,3,0}+288 \mathrm{H}_{2,3,1} \\
& +\frac{572}{3} \mathrm{H}_{3,0,0}+\frac{344}{3} \mathrm{H}_{3,1,0}+96 \mathrm{H}_{3,1,2}+192 \mathrm{H}_{3,2,0}+96 \mathrm{H}_{3,2,1}+208 \mathrm{H}_{4,0,0}+224 \mathrm{H}_{4,1,0} \\
& +96 \mathrm{H}_{4,1,1}+454 \mathrm{H}_{0,0,0,0}-224 \mathrm{H}_{0,0,0,0} \zeta_{2}+\frac{4745}{9} \mathrm{H}_{1,0,0,0}-368 \mathrm{H}_{1,0,0,0} \zeta_{2}-192 \mathrm{H}_{1,1,0,0} \zeta_{2} \\
& +256 \mathrm{H}_{1,1,1,0} \zeta_{2}-256 \mathrm{H}_{1,1,1,3}+256 \mathrm{H}_{1,1,3,0}+256 \mathrm{H}_{1,1,3,1}+\frac{176}{3} \mathrm{H}_{1,2,0,0}+96 \mathrm{H}_{1,2,2,0} \\
& +176 \mathrm{H}_{1,3,0,0}+160 \mathrm{H}_{1,3,1,0}+124 \mathrm{H}_{2,0,0,0}+\frac{176}{3} \mathrm{H}_{2,1,0,0}+96 \mathrm{H}_{2,1,2,0}+96 \mathrm{H}_{2,2,0,0} \\
& +96 \mathrm{H}_{2,2,1,0}+112 \mathrm{H}_{3,0,0,0}+112 \mathrm{H}_{3,1,0,0}+96 \mathrm{H}_{3,1,1,0}+\frac{520}{3} \mathrm{H}_{0,0,0,0,0}+\frac{560}{3} \mathrm{H}_{1,0,0,0,0} \\
& -\frac{160}{3} \mathrm{H}_{1,1,0,0,0}-48 \mathrm{H}_{1,2,0,0,0}+128 \mathrm{H}_{2,0,0,0,0}-48 \mathrm{H}_{2,1,0,0,0}+80 \mathrm{H}_{0,0,0,0,0,0} \\
& +160 \mathrm{H}_{1,0,0,0,0,0}+64 \mathrm{H}_{1,1,0,0,0,0}-320 \mathrm{H}_{1,1,1,0,0,0}+\frac{24211}{54} \mathrm{H}_{0}-160 \mathrm{H}_{0} \zeta_{5}+\frac{538}{3} \mathrm{H}_{0} \zeta_{4} \\
& +\frac{193}{3} \mathrm{H}_{0} \zeta_{3}-\frac{3700}{9} \mathrm{H}_{0} \zeta_{2}+96 \mathrm{H}_{0} \zeta_{2} \zeta_{3}-80 \mathrm{H}_{1} \zeta_{5}-\frac{8}{3} \mathrm{H}_{1} \zeta_{4}+\frac{644}{9} \mathrm{H}_{1} \zeta_{3}+96 \mathrm{H}_{1} \zeta_{2} \zeta_{3} \\
& +\frac{44416}{81} \mathrm{H}_{2}+552 \mathrm{H}_{2} \zeta_{4}+\frac{272}{3} \mathrm{H}_{2} \zeta_{3}-\frac{1072}{3} \mathrm{H}_{2} \zeta_{2}+\frac{3700}{9} \mathrm{H}_{3}+80 \mathrm{H}_{3} \zeta_{3}-\frac{344}{3} \mathrm{H}_{3} \zeta_{2} \\
& \left.+\frac{6899}{9} \mathrm{H}_{4}-224 \mathrm{H}_{4} \zeta_{2}+252 \mathrm{H}_{5}+224 \mathrm{H}_{6}\right)+x\left(-\frac{184}{3} \zeta_{5}-\frac{80}{3} \zeta_{2} \zeta_{3}+\frac{796}{3} \zeta_{4}-\frac{871}{3} \zeta_{3}\right. \\
& +\frac{68435}{54} \zeta_{2}-\frac{567245}{324} \mathrm{H}_{0,0}-\frac{52}{3} \mathrm{H}_{0,0} \zeta_{3}+\frac{875}{3} \mathrm{H}_{0,0} \zeta_{2}-\frac{1015}{3} \mathrm{H}_{2,0}-\frac{944}{3} \mathrm{H}_{2,0} \zeta_{2} \\
& +254 \mathrm{H}_{2,1}-\frac{116}{3} \mathrm{H}_{2,2}+\frac{944}{3} \mathrm{H}_{2,3}-\frac{1130}{3} \mathrm{H}_{3,0}+\frac{40}{3} \mathrm{H}_{3,1}-96 \mathrm{H}_{3,2}-\frac{1060}{3} \mathrm{H}_{4,0}-216 \mathrm{H}_{4,1} \\
& -\frac{33091}{36} \mathrm{H}_{0,0,0}+428 \mathrm{H}_{0,0,0} \zeta_{2}-\frac{1223}{3} \mathrm{H}_{2,0,0}-\frac{116}{3} \mathrm{H}_{2,1,0}-56 \mathrm{H}_{2,2,0}-148 \mathrm{H}_{3,0,0} \\
& -96 \mathrm{H}_{3,1,0}-550 \mathrm{H}_{0,0,0,0}+\frac{152}{3} \mathrm{H}_{2,0,0,0}-\frac{952}{3} \mathrm{H}_{2,1,0,0}-280 \mathrm{H}_{0,0,0,0,0}-\frac{237016}{81} \mathrm{H}_{0} \\
& -422 \mathrm{H}_{0} \zeta_{4}+\frac{569}{3} \mathrm{H}_{0} \zeta_{3}+\frac{937}{3} \mathrm{H}_{0} \zeta_{2}-\frac{68435}{54} \mathrm{H}_{2}+208 \mathrm{H}_{2} \zeta_{3}+\frac{116}{3} \mathrm{H}_{2} \zeta_{2}-\frac{937}{3} \mathrm{H}_{3} \\
& \left.+96 \mathrm{H}_{3} \zeta_{2}-\frac{875}{3} \mathrm{H}_{4}-428 \mathrm{H}_{5}\right)+(1+x)\left(360 \zeta_{6}+64 \zeta_{3}^{2}-268 \mathrm{H}_{0,0} \zeta_{4}-80 \mathrm{H}_{2,0} \zeta_{3}\right. \\
& -160 \mathrm{H}_{2,1} \zeta_{3}-112 \mathrm{H}_{2,4}+112 \mathrm{H}_{3,0} \zeta_{2}-112 \mathrm{H}_{3,3}-88 \mathrm{H}_{4,2}-172 \mathrm{H}_{5,0}-176 \mathrm{H}_{5,1} \\
& +24 \mathrm{H}_{0,0,0} \zeta_{3}+112 \mathrm{H}_{2,0,0} \zeta_{2}+96 \mathrm{H}_{2,1,0} \zeta_{2}-96 \mathrm{H}_{2,1,3}-48 \mathrm{H}_{2,3,0}-32 \mathrm{H}_{2,3,1}-48 \mathrm{H}_{3,2,0} \\
& -100 \mathrm{H}_{4,0,0}-88 \mathrm{H}_{4,1,0}+168 \mathrm{H}_{0,0,0,0} \zeta_{2}+64 \mathrm{H}_{2,2,0,0}-24 \mathrm{H}_{3,0,0,0}+64 \mathrm{H}_{3,1,0,0} \\
& -16 \mathrm{H}_{2,0,0,0,0}+64 \mathrm{H}_{2,1,0,0,0}+128 \mathrm{H}_{2,1,1,0,0}-70 \mathrm{H}_{0,0,0,0,0,0}+40 \mathrm{H}_{0} \zeta_{5}-48 \mathrm{H}_{0} \zeta_{2} \zeta_{3} \\
& \left.-216 \mathrm{H}_{2} \zeta_{4}-112 \mathrm{H}_{3} \zeta_{3}+88 \mathrm{H}_{4} \zeta_{2}-168 \mathrm{H}_{6}\right)+(1-x)\left(\frac{1325809}{324}+\frac{43301}{27} \mathrm{H}_{1,0}\right. \\
& -256 \mathrm{H}_{1,0} \zeta_{3}+\frac{560}{3} \mathrm{H}_{1,0} \zeta_{2}+\frac{3224}{3} \mathrm{H}_{1,1}-448 \mathrm{H}_{1,1} \zeta_{3}+\frac{448}{3} \mathrm{H}_{1,2}-\frac{560}{3} \mathrm{H}_{1,3}-192 \mathrm{H}_{1,4}
\end{aligned}
$$




$$
\begin{aligned}
& +\frac{3920}{9} \mathrm{H}_{1,0,0}+192 \mathrm{H}_{1,0,0} \zeta_{2}+\frac{448}{3} \mathrm{H}_{1,1,0}+704 \mathrm{H}_{1,1,0} \zeta_{2}-704 \mathrm{H}_{1,1,3}+\frac{400}{3} \mathrm{H}_{1,2,0} \\
& +128 \mathrm{H}_{1,3,0}+128 \mathrm{H}_{1,3,1}+\frac{256}{3} \mathrm{H}_{1,0,0,0}+352 \mathrm{H}_{1,1,0,0}+288 \mathrm{H}_{1,2,0,0}+32 \mathrm{H}_{1,0,0,0,0} \\
& \left.-160 \mathrm{H}_{1,1,0,0,0}+576 \mathrm{H}_{1,1,1,0,0}+\frac{41504}{27} \mathrm{H}_{1}-320 \mathrm{H}_{1} \zeta_{4}-\frac{752}{3} \mathrm{H}_{1} \zeta_{3}-\frac{448}{3} \mathrm{H}_{1} \zeta_{2}\right) \\
& -\frac{424}{3} \zeta_{5}+\frac{1364}{3} \zeta_{4}-113 \zeta_{3}-\frac{11641}{6} \zeta_{2}+\frac{208}{3} \zeta_{2} \zeta_{3}+\frac{451015}{324} \mathrm{H}_{0,0}-\frac{100}{3} \mathrm{H}_{0,0} \zeta_{3} \\
& -87 \mathrm{H}_{0,0} \zeta_{2}+\frac{7715}{9} \mathrm{H}_{2,0}+\frac{784}{3} \mathrm{H}_{2,0} \zeta_{2}+\frac{1658}{3} \mathrm{H}_{2,1}+228 \mathrm{H}_{2,2}-\frac{784}{3} \mathrm{H}_{2,3}+\frac{758}{3} \mathrm{H}_{3,0} \\
& +\frac{1096}{3} \mathrm{H}_{3,1}+64 \mathrm{H}_{3,2}+\frac{140}{3} \mathrm{H}_{4,0}+200 \mathrm{H}_{4,1}+\frac{10465}{36} \mathrm{H}_{0,0,0}+60 \mathrm{H}_{0,0,0} \zeta_{2}+\frac{809}{3} \mathrm{H}_{2,0,0} \\
& +228 \mathrm{H}_{2,1,0}+40 \mathrm{H}_{2,2,0}+28 \mathrm{H}_{3,0,0}+64 \mathrm{H}_{3,1,0}-\frac{490}{3} \mathrm{H}_{0,0,0,0}+\frac{8}{3} \mathrm{H}_{2,0,0,0}+\frac{776}{3} \mathrm{H}_{2,1,0,0} \\
& -120 \mathrm{H}_{0,0,0,0,0}+\frac{217325}{81} \mathrm{H}_{0}+258 \mathrm{H}_{0} \zeta_{4}+\frac{41}{3} \mathrm{H}_{0} \zeta_{3}-\frac{8521}{9} \mathrm{H}_{0} \zeta_{2}+\frac{11641}{6} \mathrm{H}_{2}-176 \mathrm{H}_{2} \zeta_{3} \\
& -228 \mathrm{H}_{2} \zeta_{2}+\frac{8521}{9} \mathrm{H}_{3}-64 \mathrm{H}_{3} \zeta_{2}+87 \mathrm{H}_{4}-60 \mathrm{H}_{5}-\delta(1-x)\left(\frac{1379569}{5184}-\frac{24211}{27} \zeta_{2}\right. \\
& \left.+\frac{9803}{162} \zeta_{3}+\frac{9382}{9} \zeta_{4}-\frac{838}{9} \zeta_{2} \zeta_{3}-1002 \zeta_{5}-\frac{16}{3} \zeta_{3}^{2}-135 \zeta_{6}+80 \zeta_{2} \zeta_{5}-32 \zeta_{3} \zeta_{4}+560 \zeta_{7}\right),
\end{aligned}
$$

and

$$
\begin{aligned}
P_{\mathrm{L}, 1}^{(3)}(x)= & p_{\mathrm{qq}}(x)\left(-\frac{39883}{162}+128 \zeta_{5}-\frac{680}{9} \zeta_{4}-\frac{8126}{27} \zeta_{3}+\frac{13346}{81} \zeta_{2}+32 \zeta_{2} \zeta_{3}-\frac{22247}{81} \mathrm{H}_{0,0}\right. \\
& -\frac{128}{3} \mathrm{H}_{0,0} \zeta_{3}+\frac{1372}{9} \mathrm{H}_{0,0} \zeta_{2}-\frac{13346}{81} \mathrm{H}_{1,0}-64 \mathrm{H}_{1,0} \zeta_{3}+\frac{752}{9} \mathrm{H}_{1,0} \zeta_{2}-\frac{128}{3} \mathrm{H}_{1,1} \zeta_{3} \\
& -\frac{752}{9} \mathrm{H}_{1,3}-\frac{160}{3} \mathrm{H}_{1,4}-\frac{4084}{27} \mathrm{H}_{2,0}+\frac{64}{3} \mathrm{H}_{2,0} \zeta_{2}-\frac{160}{3} \mathrm{H}_{2,2}-\frac{64}{3} \mathrm{H}_{2,3}-\frac{1100}{9} \mathrm{H}_{3,0} \\
& -\frac{160}{3} \mathrm{H}_{3,1}-\frac{128}{3} \mathrm{H}_{3,2}-96 \mathrm{H}_{4,0}-\frac{224}{3} \mathrm{H}_{4,1}-\frac{725}{3} \mathrm{H}_{0,0,0}+96 \mathrm{H}_{0,0,0} \zeta_{2}-\frac{2420}{27} \mathrm{H}_{1,0,0} \\
+ & \frac{160}{3} \mathrm{H}_{1,0,0} \zeta_{2}-\frac{128}{3} \mathrm{H}_{1,1,0} \zeta_{2}+\frac{128}{3} \mathrm{H}_{1,1,3}-\frac{160}{3} \mathrm{H}_{1,2,0}-\frac{160}{3} \mathrm{H}_{1,3,0}-\frac{64}{3} \mathrm{H}_{1,3,1} \\
& -\frac{724}{9} \mathrm{H}_{2,0,0}-\frac{160}{3} \mathrm{H}_{2,1,0}-32 \mathrm{H}_{2,2,0}-\frac{176}{3} \mathrm{H}_{3,0,0}-\frac{128}{3} \mathrm{H}_{3,1,0}-\frac{368}{3} \mathrm{H}_{0,0,0,0} \\
& -\frac{724}{9} \mathrm{H}_{1,0,0,0}-\frac{32}{3} \mathrm{H}_{1,2,0,0}-16 \mathrm{H}_{2,0,0,0}-\frac{32}{3} \mathrm{H}_{2,1,0,0}-\frac{160}{3} \mathrm{H}_{0,0,0,0,0}-\frac{128}{3} \mathrm{H}_{1,0,0,0,0} \\
+ & \frac{160}{3} \mathrm{H}_{1,1,0,0,0}-\frac{85175}{324} \mathrm{H}_{0}-\frac{376}{3} \mathrm{H}_{0} \zeta_{4}-44 \mathrm{H}_{0} \zeta_{3}+\frac{1916}{9} \mathrm{H}_{0} \zeta_{2}-\frac{208}{3} \mathrm{H}_{1} \zeta_{4}-\frac{208}{9} \mathrm{H}_{1} \zeta_{3} \\
& \left.-\frac{13346}{81} \mathrm{H}_{2}-\frac{224}{3} \mathrm{H}_{2} \zeta_{3}+\frac{160}{3} \mathrm{H}_{2} \zeta_{2}-\frac{1916}{9} \mathrm{H}_{3}+\frac{128}{3} \mathrm{H}_{3} \zeta_{2}-\frac{1372}{9} \mathrm{H}_{4}-96 \mathrm{H}_{5}\right) \\
+ & x\left(-\frac{496}{3} \zeta_{4}+\frac{454}{3} \zeta_{3}-\frac{9752}{27} \zeta_{2}+\frac{44128}{81} \mathrm{H}_{0,0}+\frac{28}{3} \mathrm{H}_{0,0} \zeta_{2}+\frac{170}{3} \mathrm{H}_{2,0}+\frac{320}{3} \mathrm{H}_{2,0} \zeta_{2}\right. \\
& -92 \mathrm{H}_{2,1}+\frac{32}{3} \mathrm{H}_{2,2}-\frac{320}{3} \mathrm{H}_{2,3}+\frac{224}{3} \mathrm{H}_{3,0}+\frac{32}{3} \mathrm{H}_{3,1}+\frac{2339}{9} \mathrm{H}_{0,0,0}+\frac{428}{3} \mathrm{H}_{2,0,0} \\
+ & \frac{32}{3} \mathrm{H}_{2,1,0}+\frac{376}{3} \mathrm{H}_{0,0,0,0}+\frac{304}{3} \mathrm{H}_{2,1,0,0}+\frac{115273}{108} \mathrm{H}_{0}-\frac{236}{3} \mathrm{H}_{0} \zeta_{3}-\frac{74}{3} \mathrm{H}_{0} \zeta_{2}+\frac{9752}{27} \mathrm{H}_{2}
\end{aligned}
$$




$$
\begin{aligned}
& \left.-\frac{32}{3} \mathrm{H}_{2} \zeta_{2}+\frac{74}{3} \mathrm{H}_{3}-\frac{28}{3} \mathrm{H}_{4}\right)+(1+x)\left(-124 \zeta_{6}+\frac{88}{3} \zeta_{5}-32 \zeta_{3}^{2}-\frac{16}{3} \zeta_{2} \zeta_{3}+\frac{64}{3} \mathrm{H}_{0,0} \zeta_{3}\right. \\
& +32 \mathrm{H}_{2,0} \zeta_{3}+64 \mathrm{H}_{2,1} \zeta_{3}+32 \mathrm{H}_{2,4}-32 \mathrm{H}_{3,0} \zeta_{2}+32 \mathrm{H}_{3,3}+\frac{88}{3} \mathrm{H}_{4,0}-48 \mathrm{H}_{0,0,0} \zeta_{2} \\
& -32 \mathrm{H}_{2,0,0} \zeta_{2}-64 \mathrm{H}_{2,1,0} \zeta_{2}+64 \mathrm{H}_{2,1,3}+8 \mathrm{H}_{3,0,0}-\frac{32}{3} \mathrm{H}_{2,0,0,0}-32 \mathrm{H}_{2,2,0,0}-32 \mathrm{H}_{3,1,0,0} \\
& \left.+40 \mathrm{H}_{0,0,0,0,0}-64 \mathrm{H}_{2,1,1,0,0}+16 \mathrm{H}_{0} \zeta_{4}+64 \mathrm{H}_{2} \zeta_{4}+32 \mathrm{H}_{3} \zeta_{3}+48 \mathrm{H}_{5}\right)+(1-x)\left(-\frac{136319}{81}\right. \\
& -\frac{12148}{27} \mathrm{H}_{1,0}+96 \mathrm{H}_{1,0} \zeta_{3}-\frac{320}{3} \mathrm{H}_{1,0} \zeta_{2}-\frac{872}{3} \mathrm{H}_{1,1}+192 \mathrm{H}_{1,1} \zeta_{3}-\frac{64}{3} \mathrm{H}_{1,2}+\frac{320}{3} \mathrm{H}_{1,3} \\
& +96 \mathrm{H}_{1,4}-\frac{1036}{9} \mathrm{H}_{1,0,0}-96 \mathrm{H}_{1,0,0} \zeta_{2}-\frac{64}{3} \mathrm{H}_{1,1,0}-192 \mathrm{H}_{1,1,0} \zeta_{2}+192 \mathrm{H}_{1,1,3}-\frac{64}{3} \mathrm{H}_{1,2,0} \\
& -\frac{16}{3} \mathrm{H}_{1,0,0,0}-128 \mathrm{H}_{1,1,0,0}-96 \mathrm{H}_{1,2,0,0}-192 \mathrm{H}_{1,1,1,0,0}-\frac{11416}{27} \mathrm{H}_{1}+192 \mathrm{H}_{1} \zeta_{4} \\
& \left.+\frac{320}{3} \mathrm{H}_{1} \zeta_{3}+\frac{64}{3} \mathrm{H}_{1} \zeta_{2}+96 \mathrm{H}_{2} \zeta_{3}\right)-\frac{224}{3} \zeta_{4}-38 \zeta_{3}+\frac{1616}{3} \zeta_{2}-\frac{38036}{81} \mathrm{H}_{0,0}+20 \mathrm{H}_{0,0} \zeta_{2} \\
& -\frac{1954}{9} \mathrm{H}_{2,0}-\frac{256}{3} \mathrm{H}_{2,0} \zeta_{2}-\frac{404}{3} \mathrm{H}_{2,1}-32 \mathrm{H}_{2,2}+\frac{256}{3} \mathrm{H}_{2,3}-\frac{128}{3} \mathrm{H}_{3,0}-\frac{160}{3} \mathrm{H}_{3,1} \\
& -\frac{473}{9} \mathrm{H}_{0,0,0}-\frac{116}{3} \mathrm{H}_{2,0,0}-32 \mathrm{H}_{2,1,0}+\frac{152}{3} \mathrm{H}_{0,0,0,0}-\frac{272}{3} \mathrm{H}_{2,1,0,0}-\frac{340325}{324} \mathrm{H}_{0} \\
& -\frac{44}{3} \mathrm{H}_{0} \zeta_{3}+\frac{2090}{9} \mathrm{H}_{0} \zeta_{2}-\frac{1616}{3} \mathrm{H}_{2}+32 \mathrm{H}_{2} \zeta_{2}-\frac{2090}{9} \mathrm{H}_{3}-20 \mathrm{H}_{4}+\delta(1-x)\left(\frac{353}{3}\right. \\
& \left.-\frac{85175}{162} \zeta_{2}-\frac{137}{9} \zeta_{3}+\frac{16186}{27} \zeta_{4}-\frac{584}{9} \zeta_{2} \zeta_{3}-\frac{248}{3} \zeta_{5}-\frac{16}{3} \zeta_{3}^{2}-144 \zeta_{6}\right) .
\end{aligned}
$$

The $n_{f}^{2}$ and $n_{f}^{3}$ contributions to eq. (4.6) are given by eqs. (4.6) and (4.12) of ref. [32].

Disregarding terms that vanish for $x \rightarrow 1$, the large- $x$ behaviour of $P_{\mathrm{ns}, \mathrm{L}}^{(3)}(x)$ can be written as

$$
P_{\mathrm{ns}, \mathrm{L}}^{(n-1)}(x)=\frac{A_{L, n}}{(1-x)_{+}}+B_{L, n} \delta(1-x)+C_{L, n} \ln (1-x)-A_{L, n}+\widetilde{D}_{L, n}
$$

in terms of the coefficients specified in eqs. (3.8), (3.9) and (3.11) above. The numerical values of the coefficients of the small- $x$ logarithms, $\ln ^{k} x$ with $k=1, \ldots, 6$, can be read off from eq. (4.11) below. All six logarithms and the constant contribution for $x \rightarrow 0$ are required for a good approximation to the splitting functions at small $x$-values relevant for collider physics.

In view of the length and complexity of the exact expressions (4.7) and (4.8) it is useful to have at one's disposal also compact approximate representations involving, besides powers of $x$, only simple functions like the plus-distribution and the end-point logarithms

$$
\mathcal{D}_{0}=1 /(1-x)_{+}, \quad x_{1}=1-x, \quad L_{1}=\ln (1-x), \quad L_{0}=\ln x .
$$

Such approximations can be readily used in $N$-space evolution programmes, see, e.g., ref. [106]. The results (4.7) and (4.8) can be parametrized with a high accuracy (of $0.1 \%$ 
or better) as

$$
\begin{aligned}
C_{F} n_{c}^{3} P_{\mathrm{L}, 0}^{(3)}(x)+C_{F} n_{c}^{2} n_{f} P_{\mathrm{L}, 1}^{(3)}(x)= \\
21209.02 \mathcal{D}_{0}+(25796.09-1.0) \delta\left(x_{1}\right)+19069.80 L_{1}-29733.85 \\
+25000\left(x_{1}\left(3.5242+8.3679 x-1.2395 x^{2}+1.7423 x^{3}\right)+11.916 x L_{0}\right. \\
\left.\quad-0.2237 x L_{0}^{2}-0.0129 x L_{0}^{3}+11.937 x_{1} L_{1}+13.955 L_{0} L_{1}\right) \\
+51671.33 L_{0}+17120.95 L_{0}^{2}+2863.226 L_{0}^{3}+297.8255 L_{0}^{4}+16 L_{0}^{5}+1 / 2 L_{0}^{6} \\
+n_{f}\left(-5179.372 \mathcal{D}_{0}-(5818.637+0.35) \delta\left(x_{1}\right)-3079.761 L_{1}+8115.605\right. \\
\quad+2500\left(x_{1}\left(-7.4077+4.5141 x-1.0069 x^{2}+0.7641 x^{3}\right)+8.4211 x_{1} L_{1}\right. \\
\left.\quad+7.5633 L_{0} L_{1}+7.5236 x L_{0}+0.2208 x L_{0}^{2}+0.05712 x L_{0}^{3}\right) \\
\left.\quad-9239.374 L_{0}-2917.312 L_{0}^{2}-430.5308 L_{0}^{3}-36 L_{0}^{4}-4 / 3 L_{0}^{5}\right) .
\end{aligned}
$$

Here the exact large- $x$ and small- $x$ coefficients have been rounded to seven significant figures. The brackets multiplied by 25000 and 2500 have been obtained by fits to the exact expressions at $10^{-6} \leq x \leq 1-10^{-6}$. The small shifts of the $\delta(1-x)$ fine-tune the accuracy of the resulting low moments and of the convolutions with the quark distributions. The required evaluation of the HPLs has been performed using a weight- 6 extension of ref. [107] and the program of ref. [108], which return identical results at the accuracy considered here.

For the corresponding non-leading contributions in the large- $n_{c}$ limit, denoted by the subscript $N$ in eqs. (3.1) and (3.2) above, we are for now limited to approximations analogous to (but more accurate than) those once constructed at three loops [48-50]. For the $n_{f}^{0}$ and $n_{f}^{1}$ parts of $P_{\mathrm{ns}}^{(3)+}(x)$ we employ an ansatz consisting of

- the two large- $x$ parameters $A_{4}$ and $B_{4}$, cf. eqs. (2.17) and (4.9),

- two of three suppressed large- $x$ logarithms $(1-x) \ln ^{k}(1-x), k=1,2,3$,

- one of ten two-parameter polynomials in $x$ that vanish for $x \rightarrow 1$,

- two of the three unknown small- $x$ logarithms $\ln ^{k} x, k=1,2,3$.

The parameters of the 90 resulting trial functions are determined from the eight available moments, and then two representatives as chosen that indicate the remaining uncertainty. The result of this process is illustrated in figures 5 and 6 .

Supplementing the approximations $A$ and $B$ in figures 5 and 6 by accurate parametrizations of the complete $n_{f}^{2}$ results of ref. [32] and the exact (but numerically truncated) $n_{f}^{3}$ expressions in a non-HPL notation, we obtain

$$
\begin{aligned}
P_{\mathrm{ns}, \mathrm{A}}^{(3)+}(x)= & C_{F} n_{c}^{3} P_{\mathrm{L}, 0}^{(3)}(x)+C_{F} n_{c}^{2} n_{f} P_{\mathrm{L}, 1}^{(3)}(x)+P_{L n_{f}}^{(3)+}(x) \\
& -507.152\left(\mathcal{D}_{0}-1\right)-2405.03 \delta\left(x_{1}\right)-1777.27 L_{1}^{2} x_{1}-204.183 L_{1}^{3} x_{1} \\
& +3948.16 x_{1}-2464.61\left(2 x-x^{2}\right) x_{1}-1839.44 L_{0}^{2}-402.156 L_{0}^{3}
\end{aligned}
$$



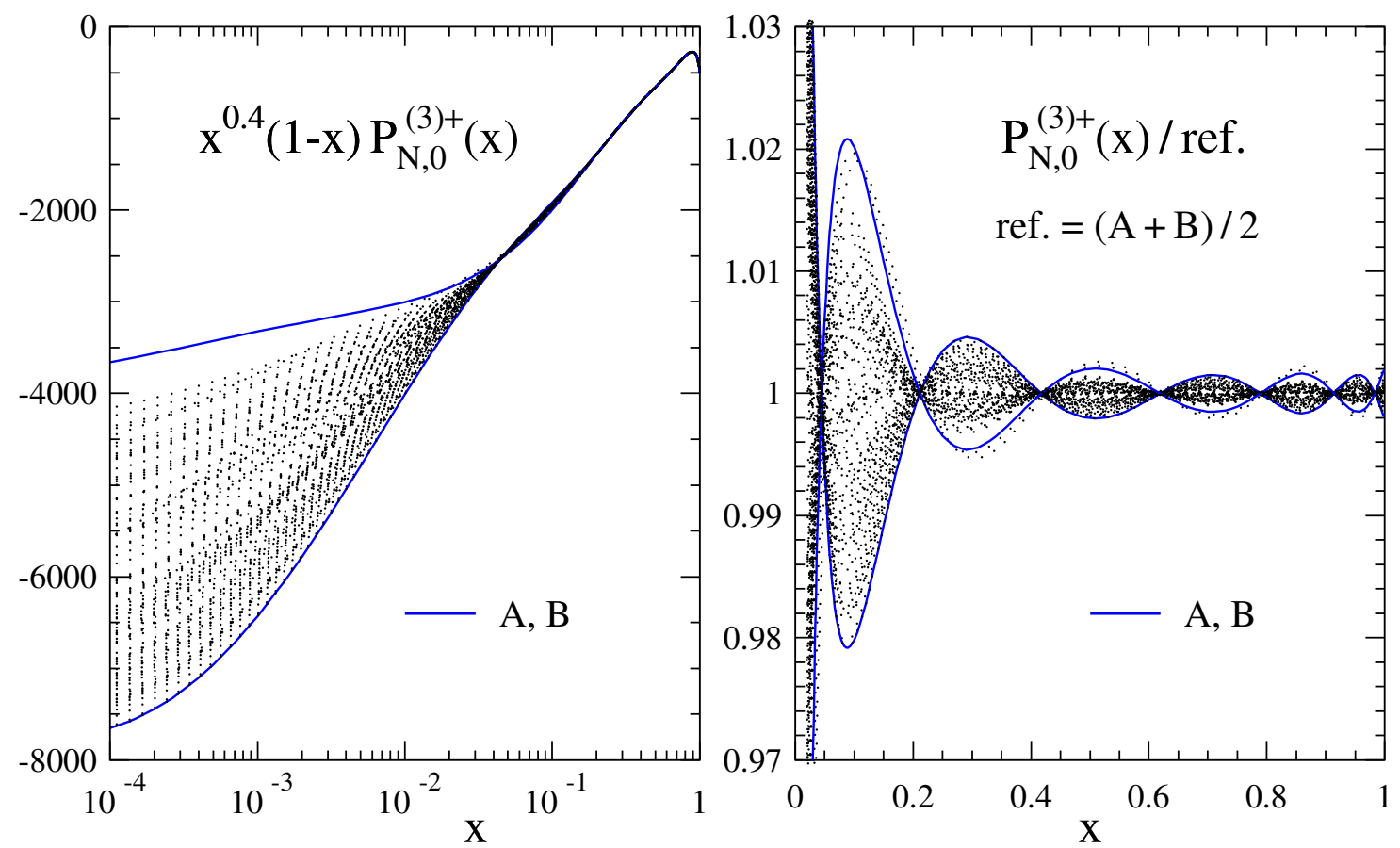

Figure 5. About 90 trial functions for the $n_{f}$-independent contribution to the non-leading $(N)$ large- $n_{c}$ part of splitting function $P_{\mathrm{ns}}^{(3)+}(x)$, multiplied by $x^{0.4}(1-x)$ for display purposes. The two functions chosen to represent the remaining uncertainty are denoted by $A$ and $B$ and shown by solid (blue) lines.

$$
\begin{aligned}
& -55.87553 L_{0}^{4}-2.831276 L_{0}^{5}-0.1488340 L_{0}^{6}-2601.749-2118.867 L_{1} \\
& +n_{f}\left(7.33927\left(\mathcal{D}_{0}-1\right)+267.965 \delta\left(x_{1}\right)-143.813 L_{1} x_{1}-18.8803 L_{1}^{3} x_{1}\right. \\
& \quad-(1116.34-1071.24 x) x x_{1}-59.3041 L_{0}^{2}-8.4620 L_{0}^{3} \\
& \left.+4.658436 L_{0}^{4}+0.2798354 L_{0}^{5}+312.1643+337.9310 L_{1}\right)
\end{aligned}
$$

and

$$
\begin{aligned}
& P_{\mathrm{ns}, \mathrm{B}}^{(3)+}(x)=C_{F} n_{c}^{3} P_{\mathrm{L}, 0}^{(3)}(x)+C_{F} n_{c}^{2} n_{f} P_{\mathrm{L}, 1}^{(3)}(x)+P_{L n_{f}}^{(3)+}(x) \\
& -505.209\left(\mathcal{D}_{0}-1\right)-2394.47 \delta\left(x_{1}\right)-173.936 L_{1}^{2} x_{1}+223.078 L_{1}^{3} x_{1} \\
& +(8698.39-10490.47 x) x x_{1}+1389.73 L_{0}+189.576 L_{0}^{2} \\
& -55.87553 L_{0}^{4}-2.831276 L_{0}^{5}-0.1488340 L_{0}^{6}-2601.749-2118.867 L_{1} \\
& +n_{f}\left(7.53662\left(\mathcal{D}_{0}-1\right)+269.028 \delta\left(x_{1}\right)-745.573 L_{1} x_{1}+8.61438 L_{1}^{3} x_{1}\right. \\
& -\left(690.151+656.386 x^{2}\right) x_{1}+133.702 L_{0}^{2}+34.0569 L_{0}^{3} \\
& \left.+4.658437 L_{0}^{4}+0.2798354 L_{0}^{5}+312.1643+337.9310 L_{1}\right)
\end{aligned}
$$



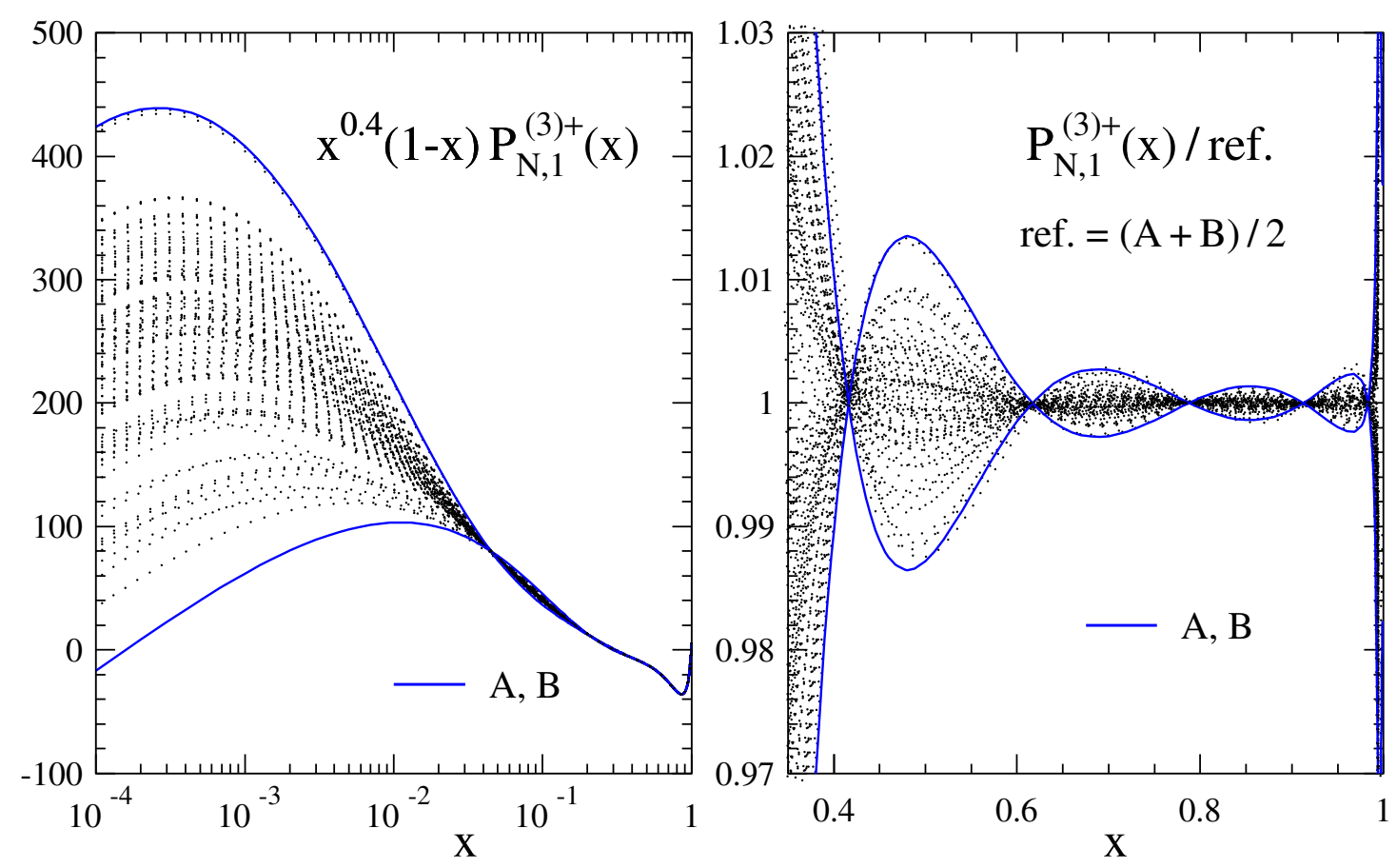

Figure 6. As figure 5, but for the $n_{f}^{1}$ contribution. The ratio in the right panel is shown for a smaller $x$-range than in figure 5 due to a sign change of the function at $x \simeq 0.3$. The large relative width of the uncertainty band close to $x=1$ is due to another change of sign at $(1-x) \simeq 0.005$.

with

$$
\begin{aligned}
P_{L n_{f}}^{(3)+}(x) & = \\
n_{f}^{2} & \left(195.5772 \mathcal{D}_{0}+26.68861 L_{1}-376.0092+(193.8554+0.0037) \delta\left(x_{1}\right)\right. \\
+ & 250\left(x_{1}\left(3.0008+0.8619 x-0.12411 x^{2}+0.31595 x^{3}\right)-0.37529 x L_{0}\right. \\
& \left.-0.21684 x L_{0}^{2}-0.02295 x L_{0}^{3}+\left(0.03394 x_{1}+0.40431 L_{0}\right) L_{1}\right) \\
& \left.+393.0056 L_{0}+112.5705 L_{0}^{2}+16.52675 L_{0}^{3}+0.7901235 L_{0}^{4}\right) \\
+ & n_{f}^{3}\left(3.272344 \mathcal{D}_{0}+3.014982 \delta\left(x_{1}\right)-2.426296-0.8460488 x\right. \\
& +\left(0.5267490 x_{1}^{-1}-3.687243+3.160494 x\right) L_{0}-\left(0.1316872\left(10 x_{1}^{-1}+1\right)\right. \\
& \left.-1.448560 x) L_{0}^{2}-\left(0.2633745 x_{1}^{-1}-0.1316870(1+x)\right) L_{0}^{3}\right) .
\end{aligned}
$$

The case of $P_{\mathrm{ns}}^{(3)-}(x)$ can be treated in the same manner, but taking into account that only its leading small- $x$ logarithm is known up to now [92]. After careful consideration, the two approximations indicating the uncertainty band in this case are chosen as

$$
P_{\mathrm{ns}, \mathrm{A}}^{(3)-}(x)=C_{F} n_{c}^{3} P_{\mathrm{L}, 0}^{(3)}(x)+C_{F} n_{c}^{2} n_{f} P_{\mathrm{L}, 1}^{(3)}(x)+P_{L n_{f}}^{(3)-}(x)
$$




$$
\begin{aligned}
& -511.228\left(\mathcal{D}_{0}-1\right)-2426.05 \delta\left(x_{1}\right)+31897.82 L_{1} x_{1}+4653.76 L_{1}^{2} x_{1} \\
& +\left(5992.88(1+2 x)+31321.44 x^{2}\right) x_{1}-1618.07 L_{0}+2.25480 L_{0}^{3} \\
& +0.4964335\left(L_{0}^{6}+6 L_{0}^{5}\right)-2601.749-2118.867 L_{1} \\
& +n_{f}\left(7.08645\left(\mathcal{D}_{0}-1\right)+266.669 \delta\left(x_{1}\right)+1856.63 L_{1} x_{1}+440.17 L_{1}^{2} x_{1}\right. \\
& \quad+\left(114.457(1+2 x)+2570.73 x^{2}\right) x_{1}-127.012 L_{0}^{2}+2.69618 L_{0}^{4} \\
& \left.\quad+312.1643+337.9310 L_{1}\right)
\end{aligned}
$$

and

$$
\begin{aligned}
& P_{\mathrm{ns}, \mathrm{B}}^{(3)-}(x)=C_{F} n_{c}^{3} P_{\mathrm{L}, 0}^{(3)}(x)+C_{F} n_{c}^{2} n_{f} P_{\mathrm{L}, 1}^{(3)}(x)+P_{L n_{f}}^{(3)-}(x) \\
& -502.481\left(\mathcal{D}_{0}-1\right)-2380.255 \delta\left(x_{1}\right)-3997.39 L_{1} x_{1}+511.567 L_{1}^{3} x_{1} \\
& +(4043.59-15386.6 x) x x_{1}+1532.96 L_{0}^{2}+31.6023 L_{0}^{3} \\
& +0.4964335\left(L_{0}^{6}+18 L_{0}^{5}\right)-2601.749-2118.867 L_{1} \\
& +n_{f}\left(7.82077\left(\mathcal{D}_{0}-1\right)+270.468 \delta\left(x_{1}\right)-1360.04 L_{1} x_{1}+38.7337 L_{1}^{3} x_{1}\right. \\
& -\left(335.995(2+x)+1605.91 x^{2}\right) x_{1}-9.76627 L_{0}^{2}+0.14218 L_{0}^{5} \\
& \left.+312.1643+337.9310 L_{1}\right)
\end{aligned}
$$

with

$$
\begin{aligned}
P_{L n_{f}}^{(3)-}(x) & = \\
n_{f}^{2} & \left(195.5772 \mathcal{D}_{0}+26.68861 L_{1}-376.0092+(193.8554+0.0037) \delta\left(x_{1}\right)\right. \\
+ & 250\left(x_{1}\left(3.2206+1.7507 x+0.13281 x^{2}+0.45969 x^{3}\right)+1.5641 x L_{0}\right. \\
& \left.-0.37902 x L_{0}^{2}-0.03248 x L_{0}^{3}+\left(2.7511 x_{1}+3.2709 L_{0}\right) L_{1}\right) \\
& \left.+437.8810 L_{0}+128.2948 L_{0}^{2}+19.59945 L_{0}^{3}+0.9876543 L_{0}^{4}\right) \\
+ & n_{f}^{3}\left(3.272344 \mathcal{D}_{0}+3.014982 \delta\left(x_{1}\right)-2.426296-0.8460488 x\right. \\
& +\left(0.5267490 x_{1}^{-1}-3.687243+3.160494 x\right) L_{0}-\left(0.1316872\left(10 x_{1}^{-1}+1\right)\right. \\
& \left.-1.448560 x) L_{0}^{2}-\left(0.2633745 x_{1}^{-1}-0.1316870(1+x)\right) L_{0}^{3}\right) .
\end{aligned}
$$

The $n_{f}^{3}$ contribution to this last equation is the same as in eq. (4.14).

Before we illustrate these results, it is useful to briefly recall the behaviour of the corresponding third-order splitting functions. This is done in figure 7 for $n_{f}=4$ flavours. The corresponding size and uncertainty bands of $P_{\mathrm{ns}}^{(3)+}(x)$ and $P_{\mathrm{ns}}^{(3)-}(x)$ are shown in figure 8 together with their large- $n_{c}$ limit. The qualitative pattern and the rough size of 

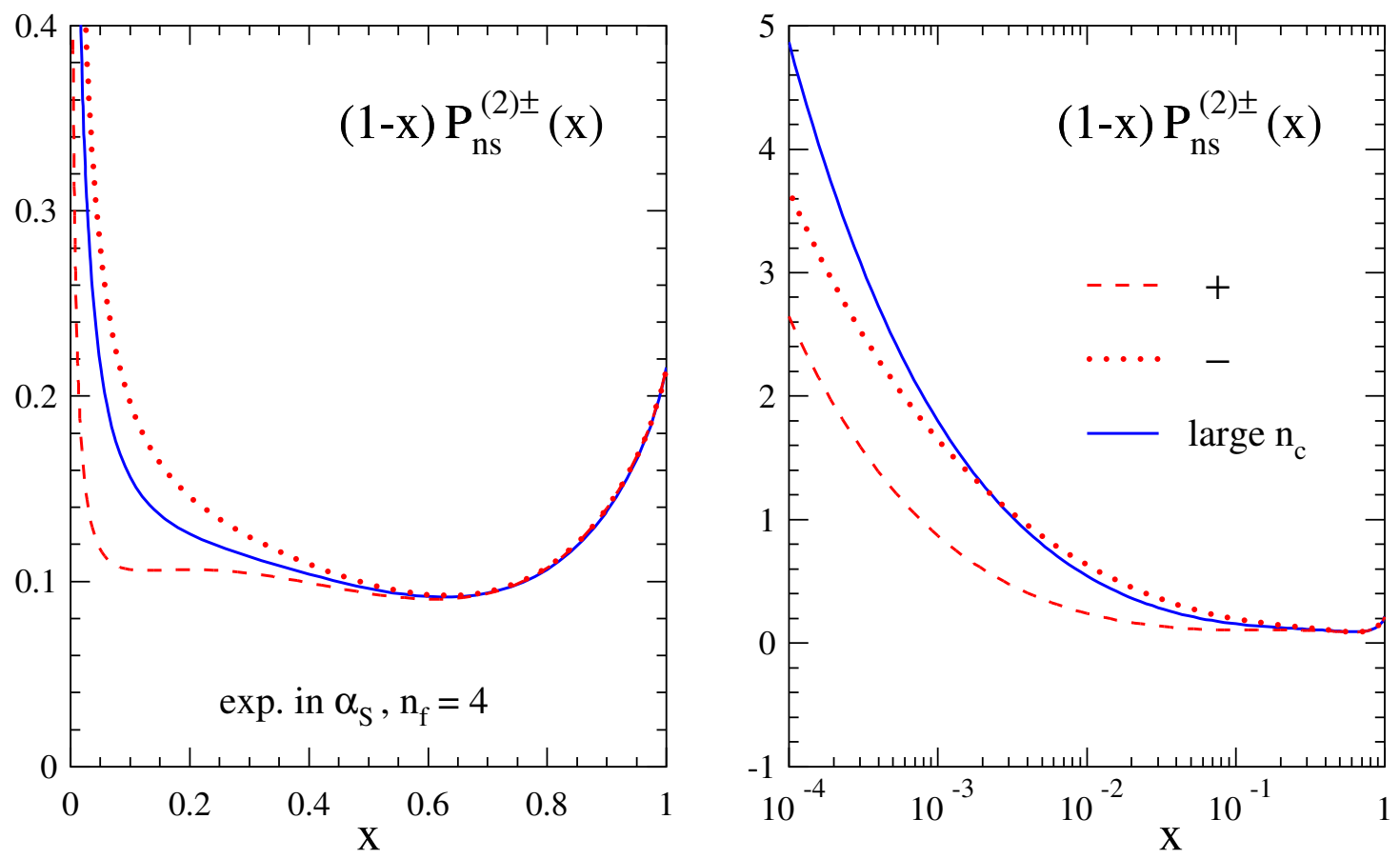

Figure 7. The three-loop splitting functions $P_{\mathrm{ns}}^{(2) \pm}(x)$ and their large- $n_{c}$ limit for QCD with four flavours.
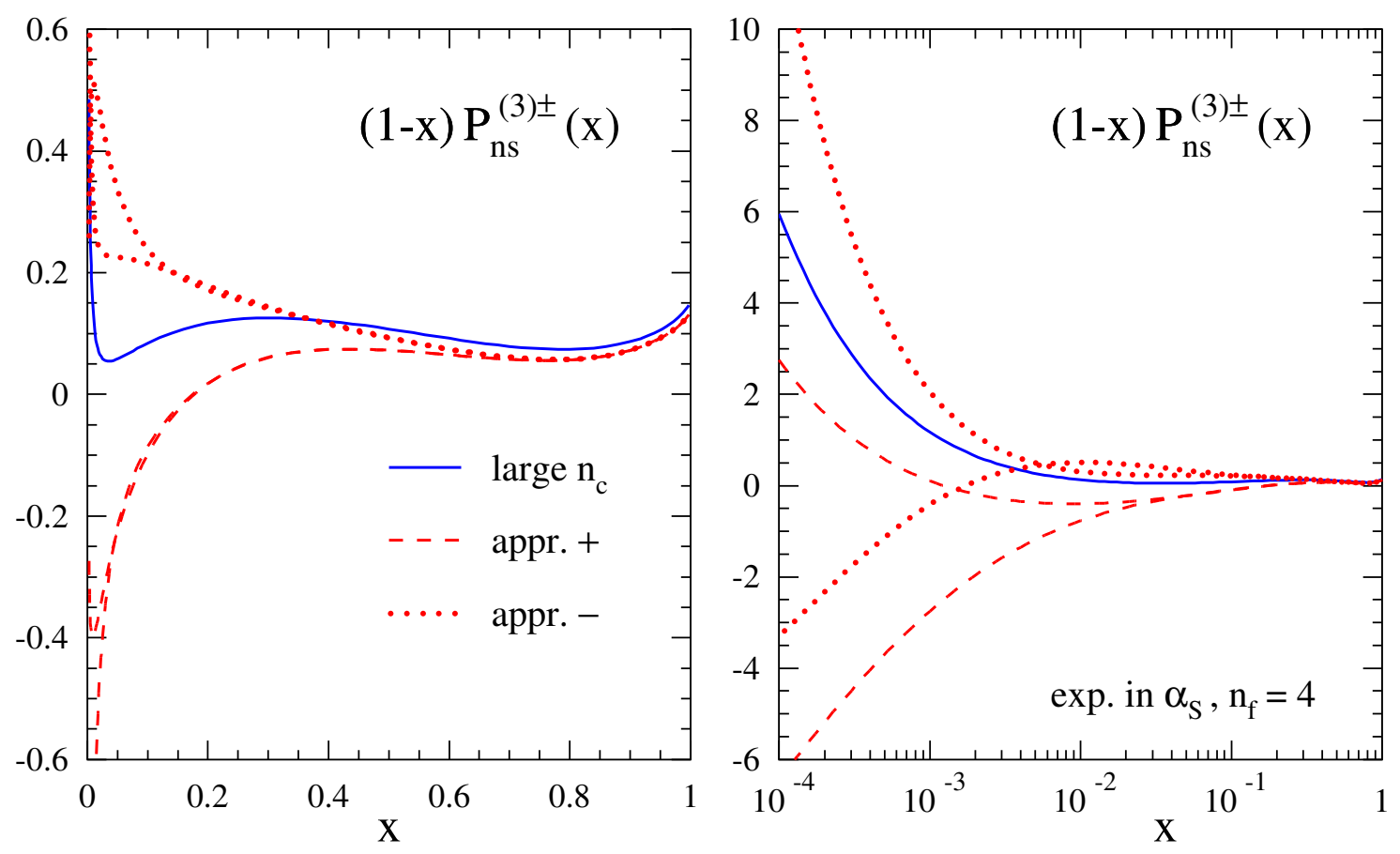

Figure 8. The four-flavour uncertainty bands for the four-loop splitting functions $P_{\mathrm{ns}}^{(3) a}(x)$ generated by eqs. (4.12) and (4.13) for $a=+$ and by eqs. (4.15) and (4.16) for $a=-$, compared to their exact large- $n_{c}$ limit. As in figure 7, the curves are scaled to an expansion in $\alpha_{\mathrm{s}}$, and only the $x<1$ contributions are shown. 
the corrections as coefficients of $\alpha_{\mathrm{s}}^{3}$ and $\alpha_{\mathrm{s}}^{4}$, respectively, are comparable in the region of $x$ for which definite conclusions can be drawn.

The four-loop 'sea' contribution $P_{\mathrm{ns}}^{(3) \mathrm{s}}(x)$ to the evolution of the total valence distribution is suppressed by two powers of $(1-x)$ for $x \rightarrow 1$, but its $n_{f}^{1}$ part is completely unknown in the small- $x$ limit. In this case, we use the nine odd moments (3.3) with a suitably modified ansatz, in which the coefficient of $\ln ^{6} x$ is varied 'by hand' over a sufficiently wide range, and the coefficients of $\ln ^{k} x, k=1, \ldots, 5$, are all determined from the moments. In this manner we obtain

$$
P_{\mathrm{ns}, \mathrm{A} / \mathrm{B}}^{(3) \mathrm{s}}(x)=n_{f} P_{1, \mathrm{~A} / \mathrm{B}}^{(3) \mathrm{s}}(x)+n_{f}^{2} P_{2}^{(3) \mathrm{s}}(x)
$$

with

$$
\begin{aligned}
P_{1, \mathrm{~A}}^{(3) \mathrm{s}}(x)= & 60.40 x_{1} L_{1}^{2}+4.685 x_{1} L_{1}^{3}+x_{1} x(4989.2-1607.73 x) \\
& +3687.6 L_{0}+3296.6 L_{0}^{2}+1271.11 L_{0}^{3}+533.44 L_{0}^{4}+97.27 L_{0}^{5}+4 L_{0}^{6}, \\
P_{1, \mathrm{~B}}^{(3) \mathrm{s}}(x)= & -254.63 x_{1} L_{1}-0.28953 x_{1} L_{1}^{3}+1030.79 x_{1} x+1266.77 x_{1}\left(2-x^{2}\right) \\
& +2987.83 L_{0}+273.05 L_{0}^{2}-923.48 L_{0}^{3}-236.76 L_{0}^{4}-33.886 L_{0}^{5}-4 L_{0}^{6}
\end{aligned}
$$

and

$$
\begin{aligned}
P_{2}^{(3) \mathrm{s}}(x)= & 19.70002 x_{1} L_{1}-3.435474 x_{1} L_{1}^{2}+250\left(x_{1}\left(-4.7656+1.6908 x+0.1703 x^{2}\right)\right. \\
& \left.-0.41652 x L_{0}+0.90777 x L_{0}^{2}+0.12478 x L_{0}^{3}+0.17155 x_{1} L_{1}+0.17191 L_{0} L_{1}\right) \\
& -647.3971 L_{0}-66.41219 L_{0}^{2}-5.353347 L_{0}^{3}-5.925926 L_{0}^{4}-0.3950617 L_{0}^{5} .
\end{aligned}
$$

The last equation is a high-accuracy parametrization, constructed in the same manner as eq. (4.11) above, of the exact result given in eq. (4.11) of ref. [32].

The trial functions considered for all three cases lead to very similar predictions for the respective next moments, i.e., $N=18$ for $P_{\mathrm{ns}}^{(3)+}(x)$, and $N=17 / 19$ for $P_{\mathrm{ns}}^{(3)-/ \mathrm{s}}(x)$. The residual uncertainty at these $N$-values is a consequence of the width of the bands at large $x$, which in turn (recall figures 5 and 6 ) is correlated with the uncertainties at smaller $x$. If the spread of the result $A$ and $B$ would underestimate the true remaining uncertainties, then a comparison with additional analytic results at these next values of $N$ should reveal a discrepancy.

In order to check this, we have extended the diagram computations of the $n_{f}^{1}$ parts of $P_{\mathrm{ns}}^{(3)-}(x)$ and $P_{\mathrm{ns}}^{(3)+}(x)$ to $N=17$ and $N=18$, respectively. The comparison of these results with the Mellin-transformed $n_{f}^{1}$ contributions to eqs. (4.12), (4.13), (4.15) and (4.16) yields

$$
\begin{aligned}
& P_{\mathrm{ns}}^{(3)-}(N=17): 194.7126372_{B}<194.7126913_{\text {exact }}<194.7127561_{A} \\
& P_{\mathrm{ns}}^{(3)+}(N=18): 195.8888792_{B}<195.8888857_{\text {exact }}<195.8888968_{A} .
\end{aligned}
$$

Similar successful checks of our approximation procedure have been carried out for the $n_{f}^{0}$ parts of $P_{\mathrm{ns}}^{(3) \pm}(x)$ and the $n_{f}^{1}$ part of $P_{\mathrm{ns}}^{(3) \mathrm{s}}(x)$ by deriving less accurate approximations 
using one fewer moment and comparing the results to the now unused highest calculated moments. As far as we can see from this and other checks, our approximation procedure, which is of course not mathematically rigorous, does not underestimate the remaining uncertainties.

\section{$5 \quad$ Numerical implications}

We are now ready to address two important applications of our new fourth-order results. First, as already mentioned above, the large- $x$ / large- $N$ limits of the splitting functions include coefficients that are relevant beyond the evolution of parton distributions: the (light-like) four-loop cusp anomalous dimension $A_{4}$ and the $\delta(1-x)$ coefficient $B_{4}$ for quark fields. We are now able to provide approximate if rather accurate numerical results for these coefficients. The obvious second application is a (further) improvement of the perturbative stability of the evolution of the non-singlet quark distributions over a wide range in $x$.

The analytic large- $n_{c}$ expression for $A_{4}$ has been presented in eq. (3.8) above. Together with the approximate results in eqs. (4.12) and (4.13) and the known $n_{f}^{2}$ and $n_{f}^{3}$ contributions, this yields

$$
A_{4}=20702(2)-5171.9(2) n_{f}+195.5772 n_{f}^{2}+3.272344 n_{f}^{3}
$$

in QCD with $n_{f}$ quark flavours. The numbers in brackets represent the uncertainty of the preceeding digit, for which we have increased the spread due to eqs. (4.12) and (4.13) by a factor of 2. eq. (5.1) leads to

$$
A_{4}=7035(2), 3353(2), 141(2) \text { for } n_{f}=3,4,5 .
$$

For comparison: the corresponding [1/1] Padé approximants used so far are 7849, 4313 and 1553 [98]. The agreement of the actual results with these approximants would be (much) better without the contributions of the quartic group invariant (see below). A similar situation has been observed for the four-loop beta function in ref. [109]. The expansion of the cusp anomalous dimension, now to the fourth order in $\alpha_{\mathrm{s}}$, is given by the very benign series

$$
\begin{aligned}
& A_{q}\left(\alpha_{\mathrm{s}}, n_{f}=3\right)=0.42441 \alpha_{\mathrm{s}}\left(1+0.72657 \alpha_{\mathrm{s}}+0.73405 \alpha_{\mathrm{s}}^{2}+0.6647(2) \alpha_{\mathrm{s}}^{3}+\ldots\right) \\
& A_{q}\left(\alpha_{\mathrm{s}}, n_{f}=4\right)=0.42441 \alpha_{\mathrm{s}}\left(1+0.63815 \alpha_{\mathrm{s}}+0.50998 \alpha_{\mathrm{s}}^{2}+0.3168(2) \alpha_{\mathrm{s}}^{3}+\ldots\right) \\
& A_{q}\left(\alpha_{\mathrm{s}}, n_{f}=5\right)=0.42441 \alpha_{\mathrm{s}}\left(1+0.54973 \alpha_{\mathrm{s}}+0.28403 \alpha_{\mathrm{s}}^{2}+0.0133(2) \alpha_{\mathrm{s}}^{3}+\ldots\right)
\end{aligned}
$$

The corresponding results for the four-loop coefficient $B_{4}$ in eqs. (2.17) and (4.9) read

$$
B_{4}=23393(10)-5551(1) n_{f}+193.8554 n_{f}^{2}+3.014982 n_{f}^{3}
$$

and

$$
\begin{aligned}
& B_{q}\left(\alpha_{\mathrm{s}}, n_{f}=3\right)=0.31831 \alpha_{\mathrm{s}}\left(1+0.99712 \alpha_{\mathrm{s}}+1.24116 \alpha_{\mathrm{s}}^{2}+1.0791(13) \alpha_{\mathrm{s}}^{3}+\ldots\right) \\
& B_{q}\left(\alpha_{\mathrm{s}}, n_{f}=4\right)=0.31831 \alpha_{\mathrm{s}}\left(1+0.87192 \alpha_{\mathrm{s}}+0.97833 \alpha_{\mathrm{s}}^{2}+0.5649(13) \alpha_{\mathrm{s}}^{3}+\ldots\right) \\
& B_{q}\left(\alpha_{\mathrm{s}}, n_{f}=5\right)=0.31831 \alpha_{\mathrm{s}}\left(1+0.74672 \alpha_{\mathrm{s}}+0.71907 \alpha_{\mathrm{s}}^{2}+0.1085(13) \alpha_{\mathrm{s}}^{3}+\ldots\right)
\end{aligned}
$$




\begin{tabular}{|ccr|}
\hline colour factor & \multicolumn{1}{c|}{$A_{4}$} & \multicolumn{1}{c|}{$B_{4}$} \\
\hline$C_{F}^{4}$ & 0 & $197 . \pm 3$. \\
$C_{F}^{3} C_{A}$ & 0 & $-687 . \pm 10$. \\
$C_{F}^{2} C_{A}^{2}$ & 0 & $1219 . \pm 12$. \\
$C_{F} C_{A}^{3}$ & $610.3 \pm 0.3$ & $295.6 \pm 2.4$ \\
$d_{F}^{a b c d} d_{A}^{a b c d} / N_{R}$ & $-507.5 \pm 6.0$ & $-996 . \pm 45$. \\
\hline$n_{f} C_{F}^{3}$ & $-31.00 \pm 0.4$ & $81.4 \pm 2.2$ \\
$n_{f} C_{F}^{2} C_{A}$ & $38.75 \pm 0.2$ & $-455.7 \pm 1.1$ \\
$n_{f} C_{F} C_{A}^{2}$ & $-440.65 \pm 0.2$ & $-274.4 \pm 1.1$ \\
$n_{f} d_{F}^{a b c d} d_{F}^{a b c d} / N_{R}$ & $-123.90 \pm 0.2$ & $-143.5 \pm 1.2$ \\
\hline$n_{f}^{2} C_{F}^{2}$ & -21.31439 & -5.775288 \\
$n_{f}^{2} C_{F} C_{A}$ & 58.36737 & 51.03056 \\
$n_{f}^{3} C_{F}$ & 2.454258 & 2.261237 \\
\hline
\end{tabular}

Table 2. Numerical results for the large- $x$ coefficients $A_{4}$ and $B_{4}$ for the seven colour factors contributing to the $n_{f}^{0}$ and $n_{f}^{1}$ parts. For completeness also the exactly known $n_{f}^{2}$ and $n_{f}^{3}$ coefficients are included.

The dominant errors in eq. (5.1) and (5.4) are those of the $n_{f}$-independent part; its relative uncertainty is $10^{-4}$ for $A_{4}$ and about four times larger for $B_{4}$. Due to constraints by large- $N$ moments, the errors of $A_{4}$ and $B_{4}$ are fully correlated. The relative uncertainties are larger for the physically relevant values of $n_{f}$, yet the accuracy in eqs. (5.3) and 5.5) should be amply sufficient for phenomenological applications.

It may be interesting, for theoretical purposes, to consider the contributions of the individual colour factors to $A_{4}$ and $B_{4}$. By repeating the approximation procedure of the previous sections separately for each colour factor, we arrive at the corresponding results collected in table 2. Our results show that both quartic group invariants definitely contribute to the four-loop cusp anomalous dimension - an issue that has attracted some interest, see, e.g., refs. [110-114] — which means that the so-called Casimir scaling between the quark and gluon cusp anomalous dimensions, $A_{q}=C_{F} / C_{A} A_{g}$, does not hold beyond three loops. A lower value, -113.66 after conversion to our notation, results from assumptions made in ref. [115] for the coefficient of $n_{f} d_{F}^{a b c d} d_{F}^{a b c d} / N_{R}$.

We now turn to the effect of the four-loop splitting functions (4.6)-(4.21) on the evolution - specifically the logarithmic derivatives $\dot{q}_{\mathrm{ns}}^{i} \equiv d \ln q_{\mathrm{ns}}^{i} / d \ln \mu_{f}^{2}$ where $\mu_{f}$ is the factorization scale - of the non-singlet combinations $q_{\mathrm{ns}}^{ \pm, \mathrm{v}}\left(x, \mu_{f}^{2}\right)$ of the quark and anti-quark distributions. In all three cases we employ the same schematic, but characteristic model distribution

$$
x q_{\mathrm{ns}}^{ \pm, \mathrm{v}}\left(x, \mu_{0}^{2}\right)=x^{0.5}(1-x)^{3} .
$$

This facilitates a direct comparison of effects of the various contributions of the splitting 
functions. For the same reason the reference scale is specified by the order-independent value

$$
\alpha_{\mathrm{s}}\left(\mu_{0}^{2}\right)=0.2
$$

for the strong coupling constant. This value corresponds to $\mu_{0}^{2} \simeq 25 \ldots 50 \mathrm{GeV}^{2}$ for $\alpha_{\mathrm{s}}\left(M_{Z}^{2}\right)=0.114 \ldots 0.120$ beyond the leading order. In this region of the physical scale $Q^{2}$ deep-inelastic scattering has been measured both at fixed-target experiments and, for much smaller $x$, at the $e p$ collider HERA. Our default for the number of effectively massless flavours is $n_{f}=4$.

The reliability of perturbative calculations can be assessed by the relative size of the higher-order correction at a 'nominal' value of the renormalization scale $\mu_{r}$, here $\mu_{r}=\mu_{f}$, and by investigating the stability of the results under variations of $\mu_{r}$. For $\mu_{r} \neq \mu_{f}$ the inverse Mellin transform, see eq. (2.8), of the perturbative expansion (2.9) in terms of $a_{\mathrm{s}}=\alpha_{\mathrm{s}} /(4 \pi)$ has to be replaced by

$$
\begin{aligned}
P_{\mathrm{ns}}^{i}\left(\mu_{f}, \mu_{r}\right)= & a_{\mathrm{s}}\left(\mu_{r}^{2}\right) P_{\mathrm{ns}}^{(0)}+a_{\mathrm{s}}^{2}\left(\mu_{r}^{2}\right)\left(P_{\mathrm{ns}}^{(1), i}-\beta_{0} L P_{\mathrm{ns}}^{(0)}\right) \\
& +a_{\mathrm{s}}^{3}\left(\mu_{r}^{2}\right)\left(P_{\mathrm{ns}}^{(2), i}-2 \beta_{0} L P_{\mathrm{ns}}^{(1), i}-\left\{\beta_{1} L-\beta_{0}^{2} L^{2}\right\} P_{\mathrm{ns}}^{(0)}\right) \\
& +a_{\mathrm{s}}^{4}\left(\mu_{r}^{2}\right)\left(P_{\mathrm{ns}}^{(3), i}-3 \beta_{0} L P_{\mathrm{ns}}^{(2), i}-\left\{2 \beta_{1} L-3 \beta_{0}^{2} L^{2}\right\} P_{\mathrm{ns}}^{(1), i}\right. \\
& \left.\quad-\left\{\beta_{2} L-\frac{5}{2} \beta_{1} \beta_{0} L^{2}+\beta_{0}^{3} L^{3}\right\} P_{\mathrm{ns}}^{(0)}\right) \quad \text { with } \quad L=\ln \frac{\mu_{f}^{2}}{\mu_{r}^{2}} .
\end{aligned}
$$

For the $\overline{\mathrm{MS}}$ expansion coefficients $\beta_{k}$ of the beta function of QCD to $\mathrm{N}^{3} \mathrm{LO}$ see refs. [109, 116] and references therein.

In figure 9 the consequences of varying $\mu_{r}$ over the range $\frac{1}{8} \mu_{f}^{2} \leq \mu_{r}^{2} \leq 8 \mu_{f}^{2}$ are displayed for $\dot{q}_{\mathrm{ns}}^{+}$at six representative values of $x$ ranging from $x=0.8$ to $x=10^{-4}$. A clear improvement of the scale stability to $\mathrm{N}^{3} \mathrm{LO}$ is found over this whole range. Due to the small size of the four-loop contributions and the ' $x$-averaging' effect of the Mellin convolution given by

$$
\left[P_{\mathrm{ns}}^{(n)} \otimes q_{\mathrm{ns}}\right](x)=\int_{x}^{1} \frac{d y}{y} P_{\mathrm{ns}}^{(n)}(y) q_{\mathrm{ns}}\left(\frac{x}{y}\right)
$$

and its generalization for the plus-distribution contributions, the approximate results of section 4 are applicable to lower values of $x$ than one might expect from figure 8 .

The relative scale uncertainties of the $\mu_{r}$-average results, conventionally estimated by

$$
\Delta \dot{q}_{\mathrm{ns}}^{i} \equiv \frac{\max \left[\dot{q}_{\mathrm{ns}}^{i}\left(x, \mu_{r}^{2}=\frac{1}{4} \mu_{f}^{2} \ldots 4 \mu_{f}^{2}\right)\right]-\min \left[\dot{q}_{\mathrm{ns}}^{i}\left(x, \mu_{r}^{2}=\frac{1}{4} \mu_{f}^{2} \ldots 4 \mu_{f}^{2}\right)\right]}{2\left|\operatorname{average}\left[\dot{q}_{\mathrm{ns}}^{i}\left(x, \mu_{r}^{2}=\frac{1}{4} \mu_{f}^{2} \ldots 4 \mu_{f}^{2}\right)\right]\right|}
$$

is shown in the left panels of figures 10, 11 and 12 for all three cases $i=+,-$ and $\mathrm{v}$. In the corresponding right panels, the relative size of the $\mathrm{N}^{3} \mathrm{LO}$ corrections at the scale $\mu_{r}=\mu_{f}$ are compared to the relative $\mathrm{N}^{2} \mathrm{LO}$ effects. Both the relative scale uncertainties and the relative corrections have a singularity at about $x \simeq 0.07$ due to a sign change of the scaling violations $d q_{\mathrm{ns}}^{i}(x) / d \ln \mu_{f}^{2}$. 
Outside the region around $x=0.07$ where the $\mu_{f}$-derivatives are small, the remaining uncertainty of $\dot{q}_{\text {ns }}^{+}$is well below $1 \%$ down to $x \simeq 10^{-3}$ and possibly, below. The size and $\mu_{r}$-variation of the NLO and NNLO contributions are somewhat larger for $\dot{q}_{\text {ns }}^{-}$at small $x$, yet neither the $\mathrm{N}^{3} \mathrm{LO}$ correction nor its scale variation exceeds $1 \%$ in the region shown in the plot.

The case of $q_{\mathrm{ns}}^{\mathrm{v}}$, shown in figure 12 is noticeably different beyond NLO due to the appearance of the $d^{a b c} d_{a b c} n_{f}$ contribution $P_{\mathrm{ns}}^{\mathrm{s}}$ which is negligible and at large $x$ but large at small $x$ [14]: the difference of the NNLO curves of figure 11 and 12 - note the different scales for the ordinate - is caused completely by this contribution due to our choice (5.6) of the input quark distribution. Also in this case our new $\mathrm{N}^{3} \mathrm{LO}$ results leads to a considerable improvement and a remaining small- $x$ uncertainty of no more than about $2 \%$ at $x \geq 10^{-4}$.

\section{The time-like case}

The differences between the initial-state ('space-like', $\sigma=-1$ ) and the final-state ('timelike', $\sigma=1$ ) splitting functions respectively governing the evolution of the parton distributions and fragmentation functions can be expressed in terms of lower-order quantities. At $\mathrm{N}^{3} \mathrm{LO}$ they read

$$
\begin{aligned}
\delta P^{(3) i}(x) \equiv & P_{\mathrm{ns}, \sigma=1}^{(3) i}(x)-P_{\mathrm{ns}, \sigma=-1}^{(3) i}(x)= \\
=2 & \left\{\left[\ln x \cdot \bar{P}_{\mathrm{ns}}^{(2) i}\right] \otimes P_{\mathrm{ns}}^{(0)}+\left[\ln x \cdot P_{\mathrm{ns}}^{(0)}\right] \otimes \bar{P}_{\mathrm{ns}}^{(2) i}+\left[\ln x \cdot \bar{P}_{\mathrm{ns}}^{(1) i}\right] \otimes \bar{P}_{\mathrm{ns}}^{(1) i}\right\} \\
& -2 P_{\mathrm{ns}}^{(0)} \otimes\left[\ln x \cdot P_{\mathrm{ns}}^{(0)}\right]^{\otimes 3}-4\left[P_{\mathrm{ns}}^{(0)}\right]^{\otimes 2} \otimes\left[\ln x \cdot P_{\mathrm{ns}}^{(0)}\right] \otimes\left[\ln ^{2} x \cdot P_{\mathrm{ns}}^{(0)}\right] \\
& -\frac{2}{3}\left[P_{\mathrm{ns}}^{(0)}\right]^{\otimes 3} \otimes\left[\ln ^{3} x \cdot P_{\mathrm{ns}}^{(0)}\right]
\end{aligned}
$$

where we have used the short-hand notations $A^{\otimes 2} \equiv A \otimes A$ etc for the Mellin convolutions, and $\bar{P}^{(n) i}$ stands for the average of the corresponding $\sigma=1$ and $\sigma=-1$ expansion coefficients, normalized as in eq. (2.9). Eq. (6.1) has been derived in ref. [56] by generalizing results in ref. [65]; it is also a direct consequence of eq. (2.15) [67].

The resulting rather lengthy explicit expressions can be found in appendix C. Here we present parametrizations in terms of powers of $x$ and the logarithms in eq. (4.10). As above, their small- $x$ and large- $x$ coefficients are exact up to their rounding to seven digits. The accuracy of the $n_{f}^{k}$ coefficients is better than $0.1 \%$ except close to zeros. The three parametrizations are given by

$$
\begin{aligned}
\delta & P^{(3)+}(x)= \\
& -33901.87 L_{1}-32392.47+25000\left(x_{1}\left(1.2960+1.7438 x-1.0943 x^{2}-0.44064 x^{3}\right)\right. \\
& \left.+x L_{0}\left(0.6440+0.8939 L_{0}+0.21405 L_{0}^{2}\right)+L_{1}\left(2.0343 x_{1}+0.35738 L_{0}\right)\right)-10399.74 L_{0} \\
& -25718.24 L_{0}^{2}-5965.487 L_{0}^{3}-206.7846 L_{0}^{4}+4.213992 L_{0}^{5}-0.7023320 L_{0}^{6} \\
+ & n_{f}\left(5483.660 L_{1}+4975.255+250\left(x_{1}\left(-19.877-8.0977 x+12.335 x^{2}+8.1174 x^{3}\right)\right.\right. \\
& \left.+x L_{0}\left(13.617-7.8856 L_{0}-2.2491 L_{0}^{2}\right)+L_{1}\left(-20.171 x_{1}+6.571 L_{0}\right)\right)+657.5425 L_{0}
\end{aligned}
$$



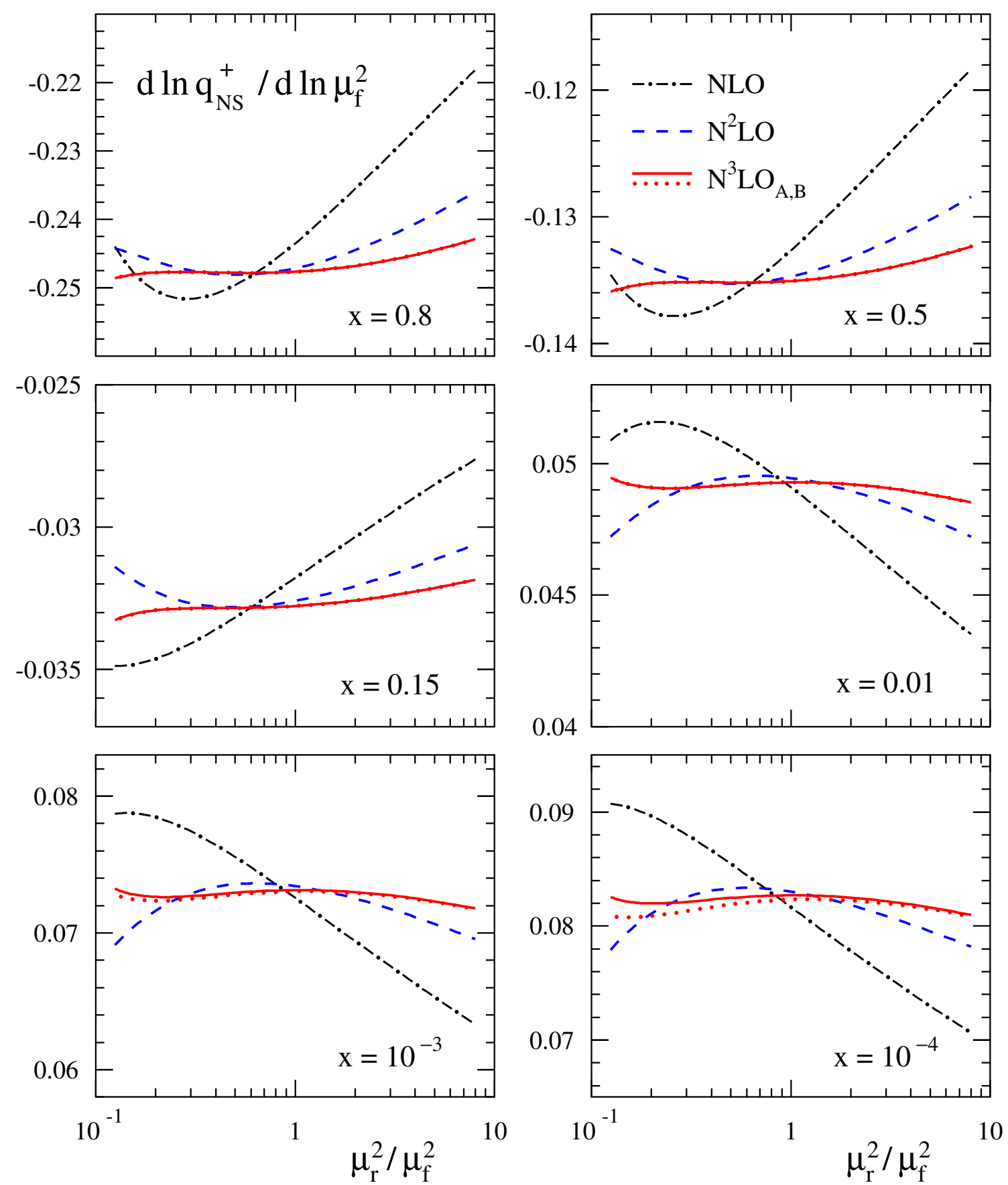

Figure 9. The dependence of the NLO, NNLO and $\mathrm{N}^{3} \mathrm{LO}$ predictions for $\dot{q}_{\mathrm{ns}}^{+} \equiv d \ln q_{\mathrm{ns}}^{+} / d \ln \mu_{f}^{2}$ on the renormalization scale $\mu_{r}$ at six typical values of $x$ for the initial conditions (5.6) and (5.7). The effect of the remaining uncertainty of the four-loop splitting function $P_{\mathrm{ns}}^{(3)+}$, indicated by the difference of the solid and dotted curves, is practically invisible except for the last two panel. 

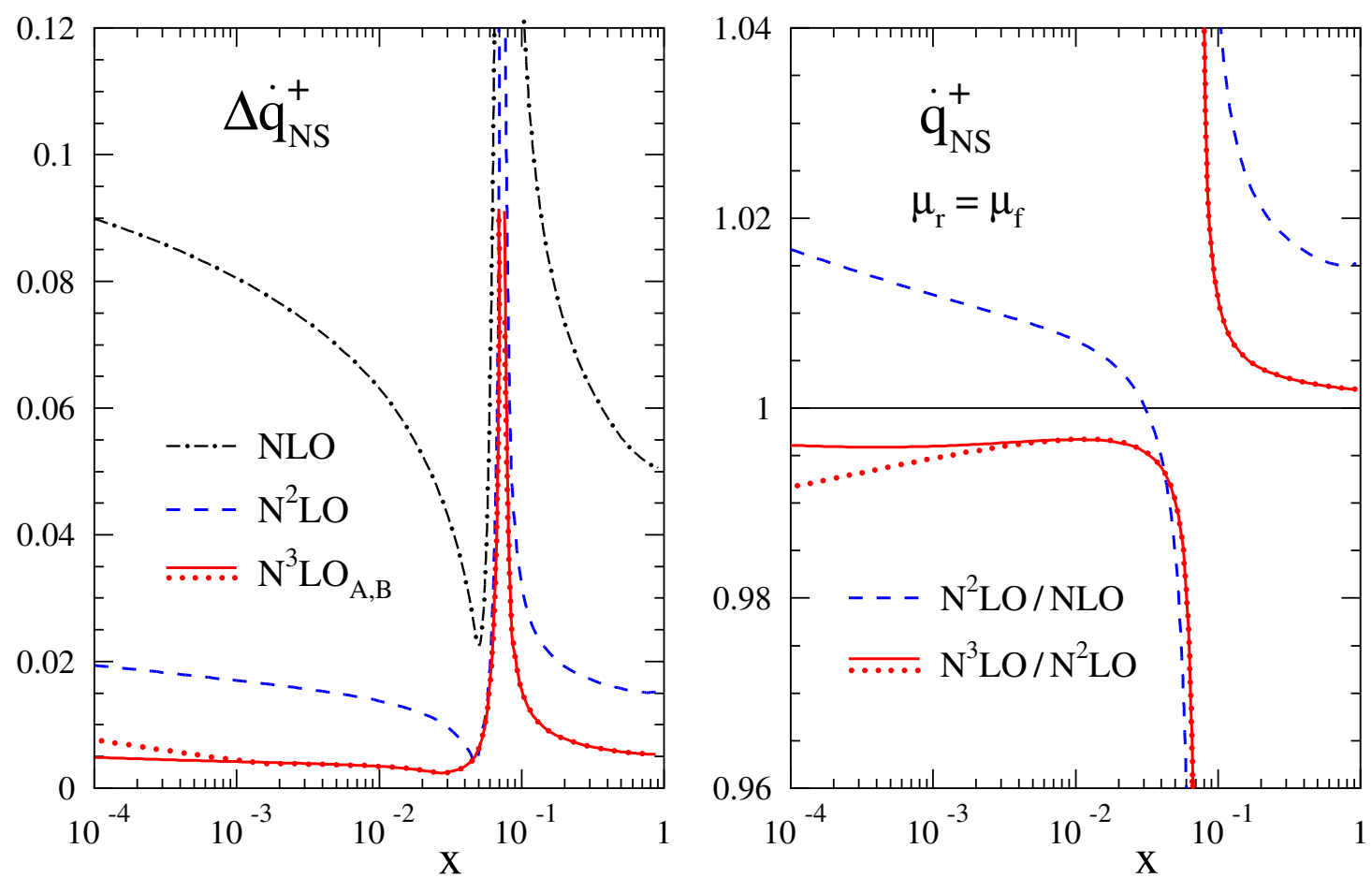

Figure 10. Left panel: the $\mu_{r}$-uncertainty of the NLO, $\mathrm{N}^{2} \mathrm{LO}$ and $\mathrm{N}^{3} \mathrm{LO}$ results for the scale derivative of $q_{\mathrm{ns}}^{+}$as obtained from the quantity $\Delta \dot{q}_{\mathrm{ns}}^{i}$ in eq. (5.10). Right panel: the relative $\mathrm{N}^{2} \mathrm{LO}$ and $\mathrm{N}^{3} \mathrm{LO}$ corrections to the logarithmic scale derivative of $q_{\mathrm{ns}}^{+}$for the characteristic non-singlet quark distribution (5.6) at $\mu_{r}=\mu_{f}$.

$$
\begin{aligned}
& \left.+3102.901 L_{0}^{2}+735.0891 L_{0}^{3}+45.82716 L_{0}^{4}\right) \\
+ & n_{f}^{2}\left(-53.37723 L_{1}-80.32433+50\left(x_{1}\left(1.6030+15.938 x-5.3145 x^{2}+1.8682 x^{3}\right)\right.\right. \\
& \left.+x L_{0}\left(13.301+2.1060 L_{0}+0.4375 L_{0}^{2}\right)+L_{1}\left(13.060 x_{1}+11.023 L_{0}\right)\right)-9.550559 L_{0} \\
& \left.-56.98805 L_{0}^{2}-21.59671 L_{0}^{3}-1.580247 L_{0}^{4}\right) \\
\delta & P^{(3)-}(x)= \\
& -33901.87 L_{1}-32392.47+25000\left(x_{1}\left(1.2892+1.4892 x-1.4262 x^{2}+0.29374 x^{3}\right)\right. \\
& \left.+x L_{0}\left(1.1307+0.17484 L_{0}+0.14894 L_{0}^{2}\right)+L_{1}\left(5.0547 x_{1}+3.4824 L_{0}\right)\right)-7307.364 L_{0} \\
& -24617.82 L_{0}^{2}-7051.323 L_{0}^{3}-665.0339 L_{0}^{4}-13.82716 L_{0}^{5}+1.035940 L_{0}^{6} \\
+ & n_{f}\left(5483.660 L_{1}+4975.255+2500\left(x_{1}\left(-1.9867-11.407 x+3.9156 x^{2}-1.6032 x^{3}\right)\right.\right. \\
& \left.+x L_{0}\left(-11.069-1.1039 L_{0}-0.2778 L_{0}^{2}\right)+L_{1}\left(-13.824 x_{1}-11.688 L_{0}\right)\right)+346.2303 L_{0} \\
& \left.+2994.194 L_{0}^{2}+780.4122 L_{0}^{3}+66.89712 L_{0}^{4}+1.580247 L_{0}^{5}\right)
\end{aligned}
$$



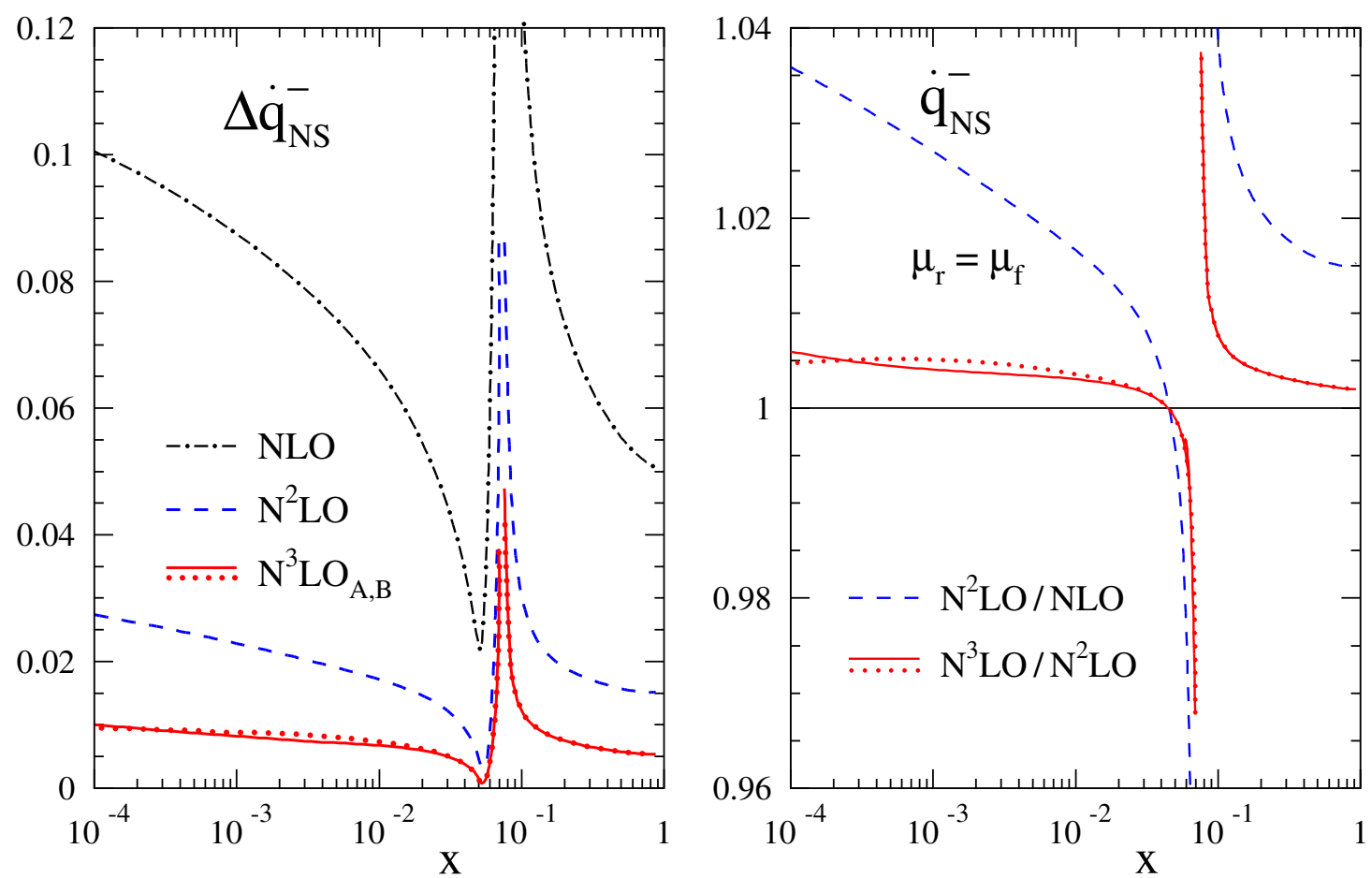

Figure 11. As figure 10, but for the scale derivative of quark minus anti-quark flavour-differences $q_{\mathrm{ns}}^{-}$.

$$
\begin{aligned}
& +n_{f}^{2}\left(-53.37723 L_{1}-80.32433+50\left(x_{1}\left(1.6030+15.938 x-5.3145 x^{2}+1.8682 x^{3}\right)\right.\right. \\
& \left.\quad+x L_{0}\left(13.301+2.1060 L_{0}+0.4375 L_{0}^{2}\right)+L_{1}\left(13.060 x_{1}+11.023 L_{0}\right)\right)-9.550559 L_{0} \\
& \left.\quad-56.98805 L_{0}^{2}-21.59671 L_{0}^{3}-1.580247 L_{0}^{4}\right) \\
& \quad \delta P^{(3) \mathrm{s}}(x)= \\
& \quad n_{f}\left(x_{1}^{2} L_{1}\left(190.3189-27.48379 L_{1}\right)+500\left(x_{1}^{2}\left(0.0597-11.761 x+3.0470 x^{2}-0.8633 x^{3}\right)\right.\right. \\
& \left.\quad+x L_{0}\left(-22.843 x_{1}-10.899 L_{0}-3.1331 L_{0}^{2}\right)+x_{1} L_{1}\left(x_{1}+L_{0}\right) 0.3835\right)+297.0894 x_{1} L_{0} \\
& \left.\quad-949.8383 L_{0}^{2}-431.7969 L_{0}^{3}+52.21778 L_{0}^{4}+7.901235 L_{0}^{5}-1.580247 L_{0}^{6}\right)
\end{aligned}
$$

The difference of the time-like and space-like splitting functions and the resulting scale derivative are illustrated in figures 13 and 14 for the most important case, $\mathrm{NS}^{+}$. The pattern is somewhat different in the time-like case, e.g., the $\mathrm{N}^{2} \mathrm{LO}$ contribution is negative at small $x$. Yet also here the perturbative expansion is 'perfectly' stable after including the $\mathrm{N}^{3} \mathrm{LO}$ corrections, with a residual uncertainty of $1 \%$ or less for $x>10^{-4}$ at our (for the time-like case, low-scale) reference point. 

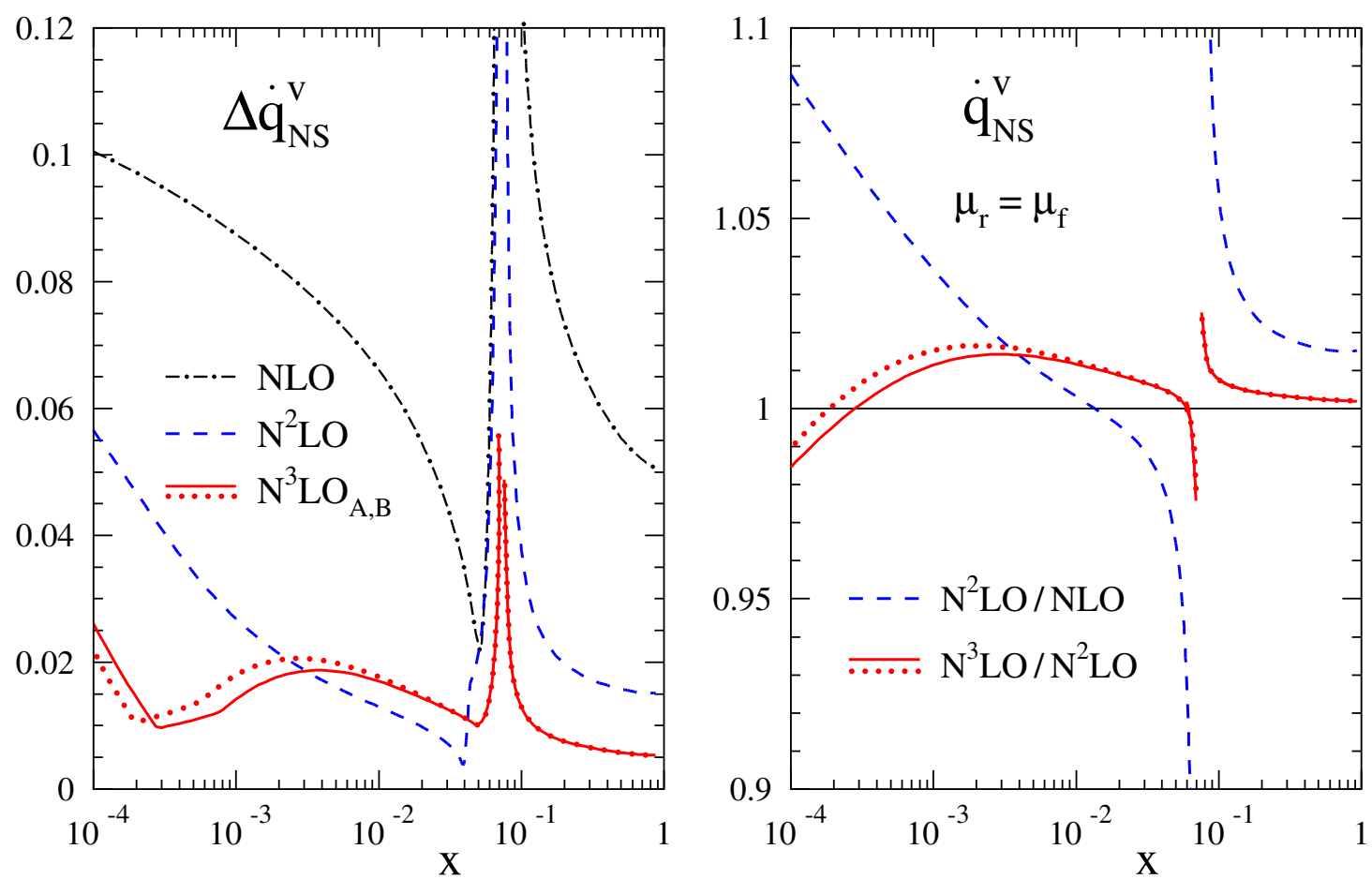

Figure 12. As figures 10 and 11, but for the total (flavour-summed) valence quark distribution $q_{\mathrm{ns}}^{\mathrm{v}}$. Here and in figures 10 and 11 the spikes close to $x=0.07$ reflect the sign-changes of $\dot{q}_{\mathrm{ns}}^{i}$ and do not constitute appreciable absolute corrections and uncertainties. All three figures refer to $n_{f}=4$ and $\alpha_{\mathrm{s}}\left(\mu_{r}^{2}=\mu_{f}^{2}\right)=0.2$.

\section{Summary and outlook}

We have presented the four-loop corrections $P_{\mathrm{ns}}^{(3) \pm, \mathrm{v}}(x)$ to all three non-singlet splitting functions in perturbative QCD. Our results, which are partly approximate but sufficiently accurate for collider-physics applications, allow to set-up and solve the QCD evolution equations for flavour non-singlet (initial-state) parton distributions (PDFs) and (final-state) fragmentation functions (FFs) at $\mathrm{N}^{3} \mathrm{LO}$. They thus provide a major step towards the consistent application of QCD factorization to theoretical predictions for $\mathrm{N}^{3} \mathrm{LO}$ cross sections with initial (final) state hadrons, as already obtained in refs. [2124, 26], which requires hard partonic cross section and PDFs (FFs) at the same order in renormalization-group improved perturbation theory.

The resulting logarithmic scale derivatives $d \ln q_{\mathrm{ns}}^{ \pm, \mathrm{v}} / d \ln \mu_{f}^{2}$ exhibit a very good convergence of the perturbative expansion. Both the four-loop corrections and the $\mathrm{N}^{3} \mathrm{LO}$ dependence on the renormalization scale $\mu_{r}$ mostly amount to as little as $1 \%$ or less (and maximally $2 \%$, for $P_{\mathrm{ns}}^{\mathrm{v}}(x)$ at small $x$ ) at momentum fractions $x>10^{-4}$ for $\alpha_{\mathrm{s}}\left(\mu_{f}^{2}\right) \simeq 0.2$.

Our results have been obtained via computations of fixed Mellin moments - to $N=20$ for the diagrams contributing in the limit of a large number $n_{c}$ of colours, and $N=16$ otherwise - for the QCD corrections to quark and anti-quark operator matrix elements (OMEs) up to four loops. After projection of all external spins and Lorentz indices, these 

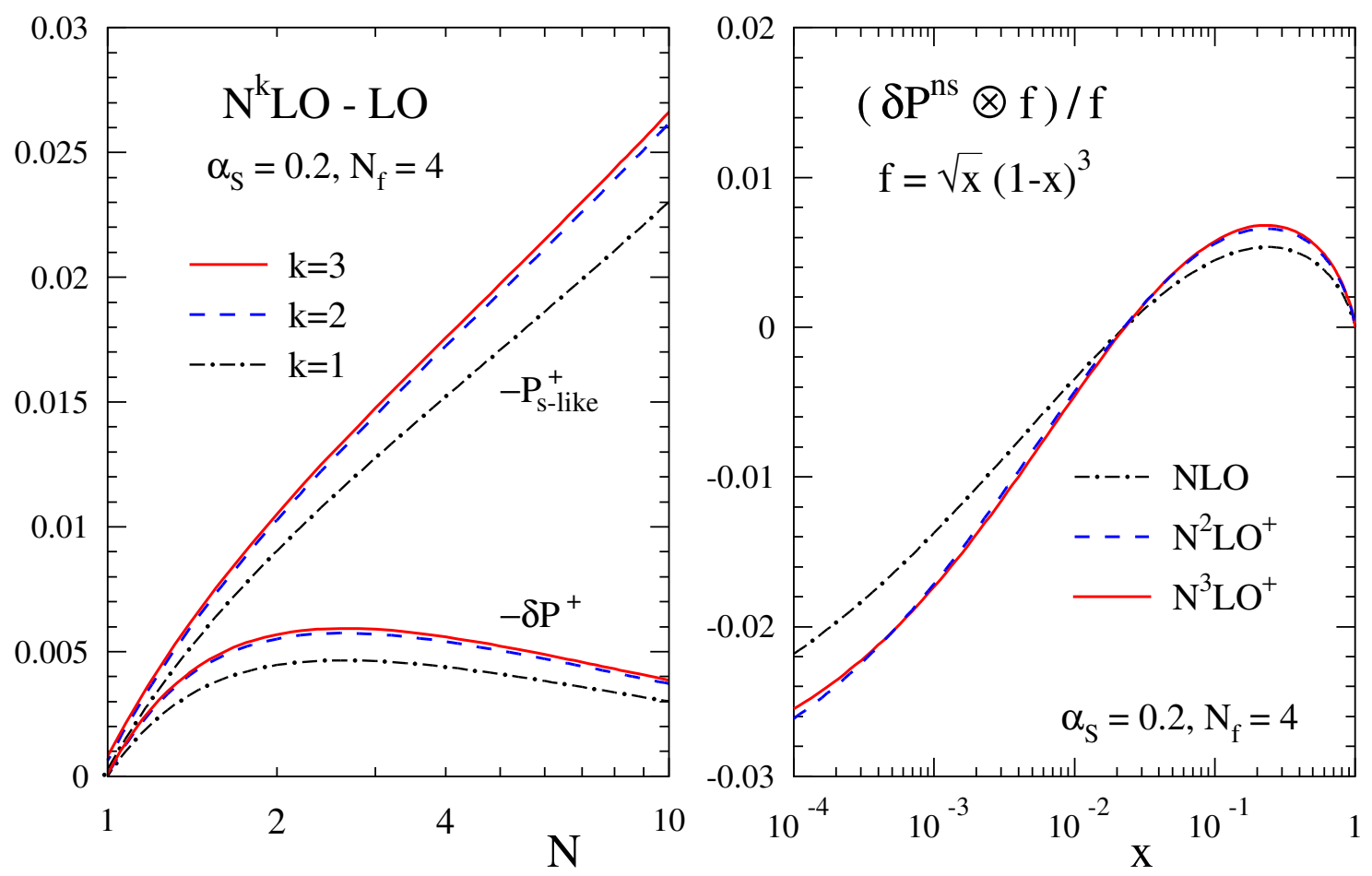

Figure 13. The perturbative expansion of the difference $\delta P^{+}=P_{\mathrm{ns}, \sigma=1}^{+}-P_{\mathrm{ns}, \sigma=-1}^{+}$of the timelike $(\sigma=1)$ and space-like $(\sigma=-1)$ non-singlet ${ }^{+}$splitting functions. Left: results in Mellin-space, compared to beyond-LO contributions in the space-like case. Right: convolution with the schematic input shape (5.6).

OMEs reduce to four-loop massless propagator integrals which can be evaluated with the FORCER program [33] in the computer algebra system Form [34-36, 74].

For the large- $n_{c}$ contributions to $P_{\mathrm{ns}}^{(3) \pm}$, these moments turn out to be sufficient for a reconstruction of the all- $N$ results in terms of harmonic sums by solving systems of Diophantine equations. Additional knowledge about the limits for $x \rightarrow 0$ and $x \rightarrow 1$, as well as the rephrasing (based on conformal symmetry) of the evolution equations in terms of a universal 'reciprocity-respecting' evolution kernel [65-67], have been instrumental in this step. Beyond the large- $n_{c}$ contributions we have used the computed Mellin moments, again supplemented by endpoint constraints, to provide approximations for the four-loop splitting functions including $x$-dependent estimates of their residual uncertainties. The latter are very small, except in the region $x<10^{-2}$. These small- $x$ uncertainties are subject to a further suppression in the actual evolution due to the convolution with the PDFs (or FFs). Due to this our results are found to be sufficiently precise for $x \gtrsim 10^{-4}$.

From the threshold limit $x \rightarrow 1$ we have been able to determine the complete cusp anomalous dimension $A_{4}$ for quarks at four loops. Our results for the $n_{f}^{0}$ and $n_{f}^{1}$ parts beyond the large- $n_{c}$ contribution are numerical, and lead to a relative accuracy of $10^{-4}$ for these coefficients in QCD, which should be amply sufficient for phenomenological applications. The break-up of $A_{4}$ in terms of individual colour factors includes non-vanishing contributions with the quartic group invariants. The exact results for $A_{4}$ of the present 

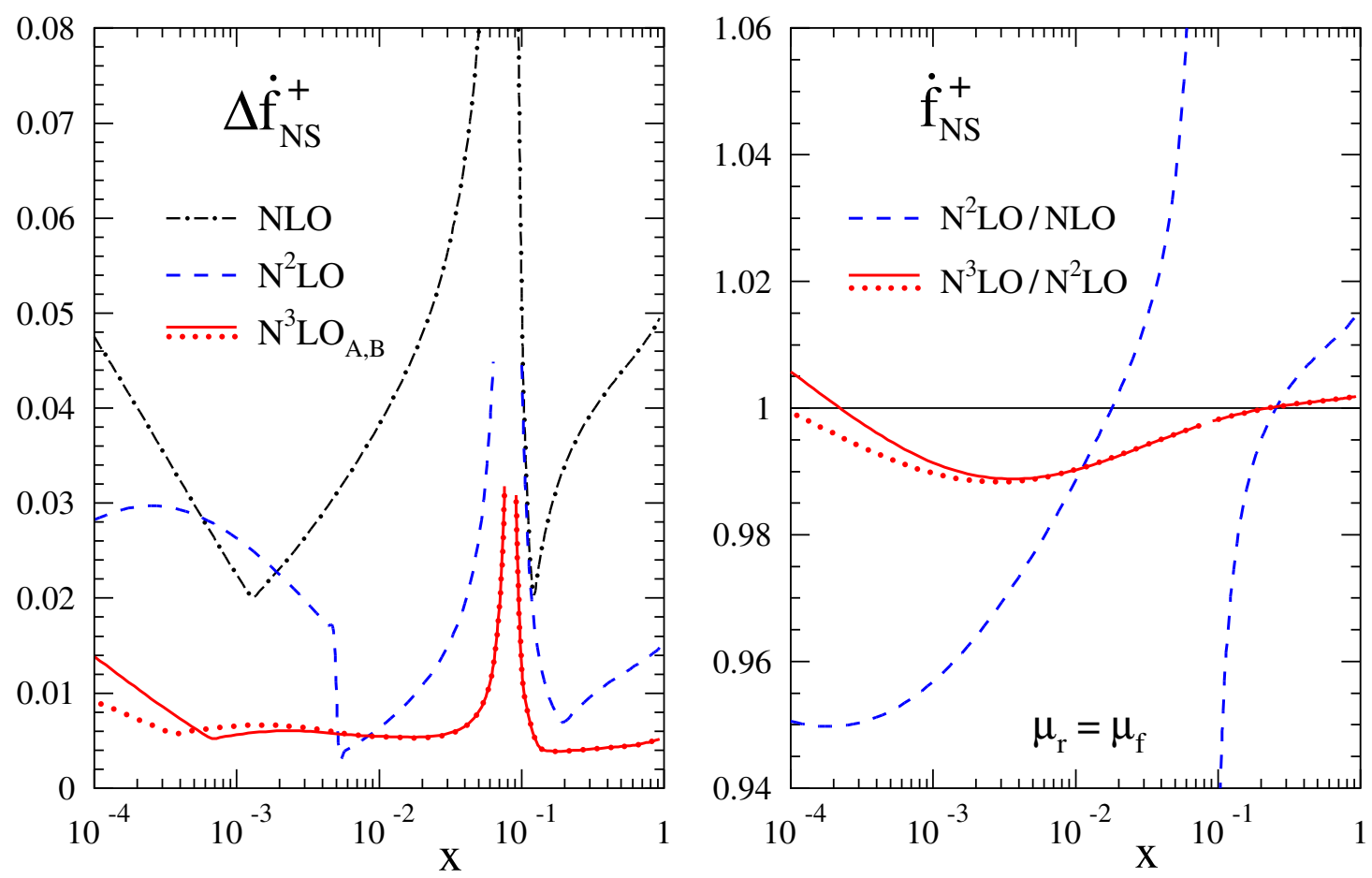

Figure 14. As figure 10, but for its time-like counterpart, the fragmentation function $f_{\mathrm{ns}}^{+}$. The same input (5.6) and (5.7) is used here, so all differences to figure 10 are due to the different $\mathrm{N}^{n>0} \mathrm{LO}$ splitting functions.

article and of ref. [32] are in agreement with the form-factor calculations in refs. [46, 47] for the planar part and refs. $[117,118]$ for the $n_{f}^{2}$ contributions. In particular, refs. [47] provided a quick confirmation of our result for the hardest part, the $n_{f}$-independent contribution, and hence of our (mathematically not completely rigorous) reconstruction of the all- $N$ expression of the large- $n_{c}$ non-singlet anomalous dimension.

The terms $B_{4}$ proportional to $\delta(1-x)$ in the four-loop splitting functions yield the universal eikonal anomalous dimension if properly combined with information on infrared singularities from the QCD form factor. This is an important ingredient for extending the threshold resummation for inclusive cross section to $\mathrm{N}^{4} \mathrm{LL}$ accuracy, i.e. (next-to-) ${ }^{4}$-leading logarithmic order.

In order to practically complete the QCD evolution equations at $\mathrm{N}^{3} \mathrm{LO}$, corresponding results are required for the singlet splitting functions at four loops, i.e., the pure-singlet quark-quark splitting function $P_{\mathrm{ps}}^{(3)}(x)$ and those involving gluons, $P_{\mathrm{qg}}^{(3)}(x), P_{\mathrm{gq}}^{(3)}(x)$ and $P_{\mathrm{gg}}^{(3)}(x)$. All these quantities are currently unknown beyond the $N=2$ and $N=4$ moments presented in ref. [31]. However, by following the approach of the present paper, it should be feasible to compute enough moments of the (theoretically much more complicated, see refs. $[12,119])$ corresponding flavour-singlet OMEs up to four loops with the FORCER program to gather sufficient information for first phenomenologically relevant approximations. We leave this topic to future research. 
Incremental improvements in the flavour non-singlet sector can be obtained by calculating more moments, which is a hard problem within the present computational set-up for almost all colour factors, and by incorporating more external information, such as, e.g., a future exact result for the four-loop cusp anomalous dimension from calculations of the photon-quark form factor.

A derivation of the exact expressions for the $n_{f}$-independent hardest parts will require, in addition, a much improved theoretical understanding. In this context it may be interesting to note that the $\zeta_{5}$ part of $\gamma_{\mathrm{ns}}^{(3) \pm}(N)$, which can be determined at all $N$ from the presently available information (see appendix D) includes a contribution

$$
-\frac{128}{3}\left\{3 C_{F}^{2} C_{A}^{2}-2 C_{F} C_{A}^{3}+12 \frac{d_{F}^{a b c d} d_{A}^{a b c d}}{N_{R}}\right\} 5 \zeta_{5}\left[S_{1}(N)\right]^{2}
$$

that vanishes in the large- $n_{c}$ limit. The resulting $\ln ^{2} N$ large- $N$ behaviour needs to be compensated by non- $\zeta_{5}$ terms, and it is tempting to identify the $5 \zeta_{5}$ in eq. (7.1) as the $\zeta_{5^{-}}$'tail' of the function

$$
f(N)=5 \zeta_{5}-2 S_{-5}+4 S_{-2} \zeta_{3}-4 S_{-2,-3}+8 S_{-2,-2,1}+4 S_{3,-2}-4 S_{4,1}+2 S_{5} .
$$

This function first occurred multiplied with positive powers of $N$ in three-loop coefficient functions of DIS in ref. [22] and resurfaced, now multiplied with $\left[S_{1}(N)\right]^{2}$ as in eq. (7.1), as the 'wrapping correction' in the anomalous dimensions in $\mathcal{N}=4$ maximally supersymmetric Yang-Mills theory [120], where it is crucial for obtaining the correct small- $x$ limit, see, e.g., ref. [121]. We thus hypothesize that eq. (7.1) represents the first glimpse of the wrapping corrections in an anomalous dimension in QCD.

FORM and FORTRAN files with our results can be obtained from the preprint server http://arXiv.org by downloading the source of this article. They are also available from the authors upon request.

\section{Acknowledgments}

S.M. would like to thank J. Gracey for useful discussions. We are grateful to T. Gehrmann and J. Blümlein for providing FORTRAN codes for harmonic polylogarithms up to weight 6, and to P. Marquard for converting of the coefficient of $n_{f} d_{F}^{a b c d} d_{F}^{a b c d} / N_{R}$ of the cusp anomalous dimension in ref. [115] to our notation. This work has been supported by the Deutsche Forschungsgemeinschaft (DFG) grant MO 1801/1-2 and SFB 676 project A3, the European Research Council (ERC) Advanced Grant 320651, HEPGAME and the U.K. Science $\mathcal{E}$ Technology Facilities Council (STFC) grant ST/L000431/1. We also are grateful for the opportunity to use most of the ulgqcd computer cluster in Liverpool which was funded by the STFC grant ST/H008837/1. The Feynman diagrams have been drawn with the packages AxODRAW [122] and JAXODRAW [123]. 


\section{A Feynman rules}

Below we present the Feynman rules for vertices arising from insertions of the operator $O_{\left\{\mu_{1}, \ldots, \mu_{N}\right\}}^{\text {ns }}$ in eq. (2.1). All momenta $p_{i}$ are flowing into the operator vertex and we use

$$
q=\sum_{i} p_{i}
$$

where $q$ is the outgoing momentum flow through the operator. The free Lorentz indices of the operator $O_{\left\{\mu_{1}, \ldots, \mu_{N}\right\}}^{\text {ns }}$ are contracted with

$$
\Delta_{\mu_{1}} \ldots \Delta_{\mu_{N}}
$$

where the vector $\Delta$ fulfils $\Delta^{2}=0$. We limit the derivation up to four additional gluons coupling to the operator, i.e., $n=6$ in figure 1. For Feynman rules with up to three additional gluons and zero momentum flow through the operator, see also ref. [72] and references therein.

The expressions for unpolarized quark operators in eqs. (A.2)-(A.6) are readily generalized to the polarized case by substituting $\Delta \rightarrow \Delta \gamma_{5}$.

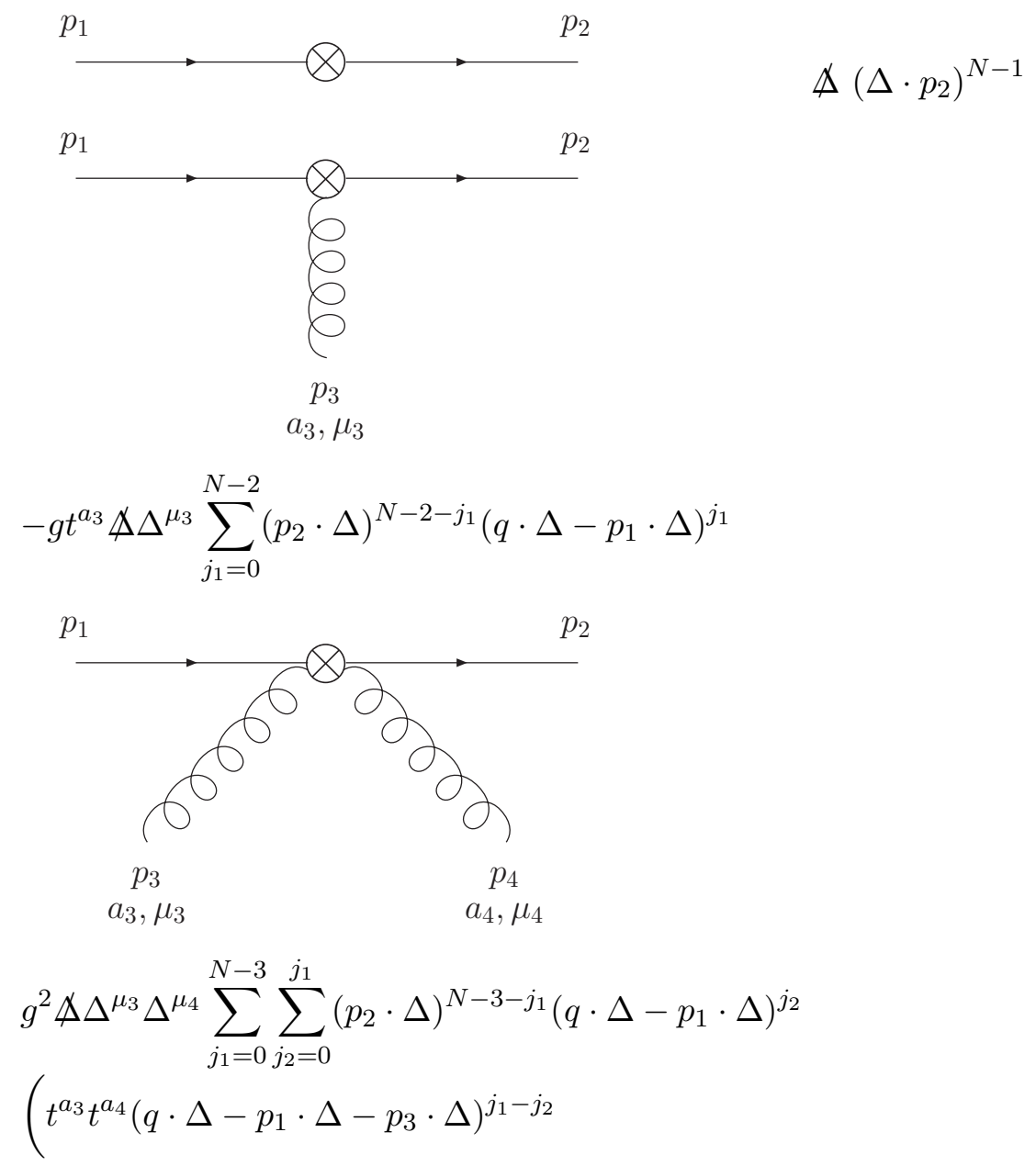




$$
\begin{aligned}
& \left.+t^{a_{4}} t^{a_{3}}\left(q \cdot \Delta-p_{1} \cdot \Delta-p_{4} \cdot \Delta\right)^{j_{1}-j_{2}}\right) \\
& \left(g^{3} \Delta \Delta^{\mu_{3}} \Delta^{\mu_{4}} \Delta \sum^{\mu_{5}} \sum_{j_{1}=0}^{N-4} \sum_{j_{2}=0}^{j_{1}} \sum_{j_{3}=0}^{j_{2}}\left(p_{2} \cdot \Delta\right)^{a_{5}}\left(q \cdot \Delta-p_{1} \cdot \Delta-p_{3} \cdot \Delta\right)^{j_{2}-j_{3}}\left(q \cdot \Delta-p_{1} \cdot \Delta-p_{3} \cdot \Delta-p_{4} \cdot \Delta\right)^{j_{1}-j_{2}}\right. \\
& +t^{a_{3}} t^{a_{5}} t^{a_{4}}\left(q \cdot \Delta-p_{1} \cdot \Delta-p_{3} \cdot \Delta\right)^{j_{2}-j_{3}}\left(q \cdot \Delta-p_{1} \cdot \Delta-p_{3} \cdot \Delta-p_{5} \cdot \Delta\right)^{j_{1}-j_{2}} \\
& +t^{a_{4}} t^{a_{3}} t^{a_{5}}\left(q \cdot \Delta-p_{1} \cdot \Delta-p_{4} \cdot \Delta\right)^{j_{2}-j_{3}}\left(q \cdot \Delta-p_{1} \cdot \Delta-p_{3} \cdot \Delta-p_{4} \cdot \Delta\right)^{j_{1}-j_{2}} \\
& +t^{a_{4}} t^{a_{5}} t^{a_{3}}\left(q \cdot \Delta-p_{1} \cdot \Delta-p_{5} \cdot \Delta\right)^{j_{2}-j_{3}}\left(q \cdot \Delta-p_{1} \cdot \Delta-p_{3} \cdot \Delta-p_{5} \cdot \Delta\right)^{j_{1}-j_{2}} \\
& +t^{a_{5}} t^{a_{3}} t^{a_{4}}\left(q \cdot \Delta-p_{1} \cdot \Delta-p_{4} \cdot \Delta\right)^{j_{2}-j_{3}}\left(q \cdot \Delta-p_{1} \cdot \Delta-p_{4} \cdot \Delta-p_{5} \cdot \Delta\right)^{j_{1}-j_{2}} \\
& \left.+t^{a_{5}} t^{a_{4}} t^{a_{3}}\left(q \cdot \Delta-p_{1} \cdot \Delta-p_{5} \cdot \Delta\right)^{j_{2}-j_{3}}\left(q \cdot \Delta-p_{1} \cdot \Delta-p_{4} \cdot \Delta-p_{5} \cdot \Delta\right)^{j_{1}-j_{2}}\right)
\end{aligned}
$$

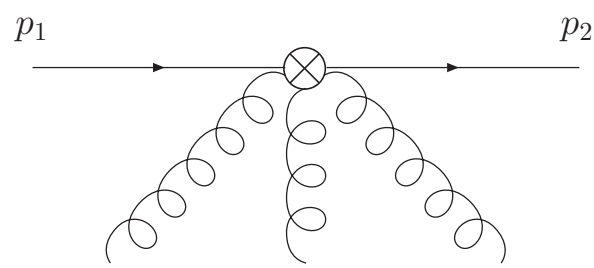

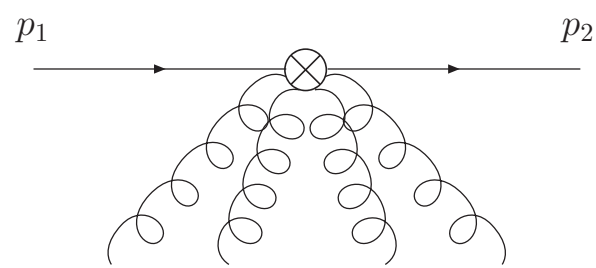

$$
\begin{array}{cccc}
p_{3} & p_{4} & p_{5} & p_{6} \\
a_{3}, \mu_{3} & a_{4}, \mu_{4} & a_{5}, \mu_{5} & a_{6}, \mu_{6}
\end{array}
$$$$
g^{4} \Delta \Delta^{\mu_{3}} \Delta^{\mu_{4}} \Delta^{\mu_{5}} \Delta^{\mu_{6}} \sum_{j_{1}=0}^{N-5} \sum_{j_{2}=0}^{j_{1}} \sum_{j_{3}=0}^{j_{2}} \sum_{j_{4}=0}^{j_{3}}\left(p_{2} \cdot \Delta\right)^{N-5-j_{1}}\left(q \cdot \Delta-p_{1} \cdot \Delta\right)^{j_{4}}
$$$$
\left(t^{a_{3}} t^{a_{4}} t^{a_{5}} t^{a_{6}}\left(q \cdot \Delta-p_{1} \cdot \Delta-p_{3} \cdot \Delta\right)^{j_{3}-j_{4}}\left(q \cdot \Delta-p_{1} \cdot \Delta-p_{3} \cdot \Delta-p_{4} \cdot \Delta\right)^{j_{2}-j_{3}}\right.
$$$$
\left(q \cdot \Delta-p_{1} \cdot \Delta-p_{3} \cdot \Delta-p_{4} \cdot \Delta-p_{5} \cdot \Delta\right)^{j_{1}-j_{2}}
$$$$
+t^{a_{3}} t^{a_{4}} t^{a_{6}} t^{a_{5}}\left(q \cdot \Delta-p_{1} \cdot \Delta-p_{3} \cdot \Delta\right)^{j_{3}-j_{4}}\left(q \cdot \Delta-p_{1} \cdot \Delta-p_{3} \cdot \Delta-p_{4} \cdot \Delta\right)^{j_{2}-j_{3}}
$$$$
\left(q \cdot \Delta-p_{1} \cdot \Delta-p_{3} \cdot \Delta-p_{4} \cdot \Delta-p_{6} \cdot \Delta\right)^{j_{1}-j_{2}}
$$$$
+t^{a_{3}} t^{a_{5}} t^{a_{4}} t^{a_{6}}\left(q \cdot \Delta-p_{1} \cdot \Delta-p_{3} \cdot \Delta\right)^{j_{3}-j_{4}}\left(q \cdot \Delta-p_{1} \cdot \Delta-p_{3} \cdot \Delta-p_{5} \cdot \Delta\right)^{j_{2}-j_{3}}
$$$$
\left(q \cdot \Delta-p_{1} \cdot \Delta-p_{3} \cdot \Delta-p_{4} \cdot \Delta-p_{5} \cdot \Delta\right)^{j_{1}-j_{2}}
$$$$
+t^{a_{3}} t^{a_{5}} t^{a_{6}} t^{a_{4}}\left(q \cdot \Delta-p_{1} \cdot \Delta-p_{3} \cdot \Delta\right)^{j_{3}-j_{4}}\left(q \cdot \Delta-p_{1} \cdot \Delta-p_{3} \cdot \Delta-p_{5} \cdot \Delta\right)^{j_{2}-j_{3}}
$$$$
\left(q \cdot \Delta-p_{1} \cdot \Delta-p_{3} \cdot \Delta-p_{5} \cdot \Delta-p_{6} \cdot \Delta\right)^{j_{1}-j_{2}}
$$$$
+t^{a_{3}} t^{a_{6}} t^{a_{4}} t^{a_{5}}\left(q \cdot \Delta-p_{1} \cdot \Delta-p_{3} \cdot \Delta\right)^{j_{3}-j_{4}}\left(q \cdot \Delta-p_{1} \cdot \Delta-p_{3} \cdot \Delta-p_{6} \cdot \Delta\right)^{j_{2}-j_{3}}
$$ 


$$
\begin{aligned}
& \left(q \cdot \Delta-p_{1} \cdot \Delta-p_{3} \cdot \Delta-p_{4} \cdot \Delta-p_{6} \cdot \Delta\right)^{j_{1}-j_{2}} \\
& +t^{a_{3}} t^{a_{6}} t^{a_{5}} t^{a_{4}}\left(q \cdot \Delta-p_{1} \cdot \Delta-p_{3} \cdot \Delta\right)^{j_{3}-j_{4}}\left(q \cdot \Delta-p_{1} \cdot \Delta-p_{3} \cdot \Delta-p_{6} \cdot \Delta\right)^{j_{2}-j_{3}} \\
& \left(q \cdot \Delta-p_{1} \cdot \Delta-p_{3} \cdot \Delta-p_{5} \cdot \Delta-p_{6} \cdot \Delta\right)^{j_{1}-j_{2}} \\
& +t^{a_{4}} t^{a_{3}} t^{a_{5}} t^{a_{6}}\left(q \cdot \Delta-p_{1} \cdot \Delta-p_{4} \cdot \Delta\right)^{j_{3}-j_{4}}\left(q \cdot \Delta-p_{1} \cdot \Delta-p_{3} \cdot \Delta-p_{4} \cdot \Delta\right)^{j_{2}-j_{3}} \\
& \left(q \cdot \Delta-p_{1} \cdot \Delta-p_{3} \cdot \Delta-p_{4} \cdot \Delta-p_{5} \cdot \Delta\right)^{j_{1}-j_{2}} \\
& +t^{a_{4}} t^{a_{3}} t^{a_{6}} t^{a_{5}}\left(q \cdot \Delta-p_{1} \cdot \Delta-p_{4} \cdot \Delta\right)^{j_{3}-j_{4}}\left(q \cdot \Delta-p_{1} \cdot \Delta-p_{3} \cdot \Delta-p_{4} \cdot \Delta\right)^{j_{2}-j_{3}} \\
& \left(q \cdot \Delta-p_{1} \cdot \Delta-p_{3} \cdot \Delta-p_{4} \cdot \Delta-p_{6} \cdot \Delta\right)^{j_{1}-j_{2}} \\
& +t^{a_{4}} t^{a_{5}} t^{a_{3}} t^{a_{6}}\left(q \cdot \Delta-p_{1} \cdot \Delta-p_{4} \cdot \Delta\right)^{j_{3}-j_{4}}\left(q \cdot \Delta-p_{1} \cdot \Delta-p_{4} \cdot \Delta-p_{5} \cdot \Delta\right)^{j_{2}-j_{3}} \\
& \left(q \cdot \Delta-p_{1} \cdot \Delta-p_{3} \cdot \Delta-p_{4} \cdot \Delta-p_{5} \cdot \Delta\right)^{j_{1}-j_{2}} \\
& +t^{a_{4}} t^{a_{5}} t^{a_{6}} t^{a_{3}}\left(q \cdot \Delta-p_{1} \cdot \Delta-p_{4} \cdot \Delta\right)^{j_{3}-j_{4}}\left(q \cdot \Delta-p_{1} \cdot \Delta-p_{4} \cdot \Delta-p_{5} \cdot \Delta\right)^{j_{2}-j_{3}} \\
& \left(q \cdot \Delta-p_{1} \cdot \Delta-p_{4} \cdot \Delta-p_{5} \cdot \Delta-p_{6} \cdot \Delta\right)^{j_{1}-j_{2}} \\
& +t^{a_{4}} t^{a_{6}} t^{a_{3}} t^{a_{5}}\left(q \cdot \Delta-p_{1} \cdot \Delta-p_{4} \cdot \Delta\right)^{j_{3}-j_{4}}\left(q \cdot \Delta-p_{1} \cdot \Delta-p_{4} \cdot \Delta-p_{6} \cdot \Delta\right)^{j_{2}-j_{3}} \\
& \left(q \cdot \Delta-p_{1} \cdot \Delta-p_{3} \cdot \Delta-p_{4} \cdot \Delta-p_{6} \cdot \Delta\right)^{j_{1}-j_{2}} \\
& +t^{a_{4}} t^{a_{6}} t^{a_{5}} t^{a_{3}}\left(q \cdot \Delta-p_{1} \cdot \Delta-p_{4} \cdot \Delta\right)^{j_{3}-j_{4}}\left(q \cdot \Delta-p_{1} \cdot \Delta-p_{4} \cdot \Delta-p_{6} \cdot \Delta\right)^{j_{2}-j_{3}} \\
& \left(q \cdot \Delta-p_{1} \cdot \Delta-p_{4} \cdot \Delta-p_{5} \cdot \Delta-p_{6} \cdot \Delta\right)^{j_{1}-j_{2}} \\
& +t^{a_{5}} t^{a_{3}} t^{a_{4}} t^{a_{6}}\left(q \cdot \Delta-p_{1} \cdot \Delta-p_{5} \cdot \Delta\right)^{j_{3}-j_{4}}\left(q \cdot \Delta-p_{1} \cdot \Delta-p_{3} \cdot \Delta-p_{5} \cdot \Delta\right)^{j_{2}-j_{3}} \\
& \left(q \cdot \Delta-p_{1} \cdot \Delta-p_{3} \cdot \Delta-p_{4} \cdot \Delta-p_{5} \cdot \Delta\right)^{j_{1}-j_{2}} \\
& +t^{a_{5}} t^{a_{3}} t^{a_{6}} t^{a_{4}}\left(q \cdot \Delta-p_{1} \cdot \Delta-p_{5} \cdot \Delta\right)^{j_{3}-j_{4}}\left(q \cdot \Delta-p_{1} \cdot \Delta-p_{3} \cdot \Delta-p_{5} \cdot \Delta\right)^{j_{2}-j_{3}} \\
& \left(q \cdot \Delta-p_{1} \cdot \Delta-p_{3} \cdot \Delta-p_{5} \cdot \Delta-p_{6} \cdot \Delta\right)^{j_{1}-j_{2}} \\
& +t^{a_{5}} t^{a_{4}} t^{a_{3}} t^{a_{6}}\left(q \cdot \Delta-p_{1} \cdot \Delta-p_{5} \cdot \Delta\right)^{j_{3}-j_{4}}\left(q \cdot \Delta-p_{1} \cdot \Delta-p_{4} \cdot \Delta-p_{5} \cdot \Delta\right)^{j_{2}-j_{3}} \\
& \left(q \cdot \Delta-p_{1} \cdot \Delta-p_{3} \cdot \Delta-p_{4} \cdot \Delta-p_{5} \cdot \Delta\right)^{j_{1}-j_{2}} \\
& +t^{a_{5}} t^{a_{4}} t^{a_{6}} t^{a_{3}}\left(q \cdot \Delta-p_{1} \cdot \Delta-p_{5} \cdot \Delta\right)^{j_{3}-j_{4}}\left(q \cdot \Delta-p_{1} \cdot \Delta-p_{4} \cdot \Delta-p_{5} \cdot \Delta\right)^{j_{2}-j_{3}} \\
& \left(q \cdot \Delta-p_{1} \cdot \Delta-p_{4} \cdot \Delta-p_{5} \cdot \Delta-p_{6} \cdot \Delta\right)^{j_{1}-j_{2}} \\
& +t^{a_{5}} t^{a_{6}} t^{a_{3}} t^{a_{4}}\left(q \cdot \Delta-p_{1} \cdot \Delta-p_{5} \cdot \Delta\right)^{j_{3}-j_{4}}\left(q \cdot \Delta-p_{1} \cdot \Delta-p_{5} \cdot \Delta-p_{6} \cdot \Delta\right)^{j_{2}-j_{3}} \\
& \left(q \cdot \Delta-p_{1} \cdot \Delta-p_{3} \cdot \Delta-p_{5} \cdot \Delta-p_{6} \cdot \Delta\right)^{j_{1}-j_{2}} \\
& +t^{a_{5}} t^{a_{6}} t^{a_{4}} t^{a_{3}}\left(q \cdot \Delta-p_{1} \cdot \Delta-p_{5} \cdot \Delta\right)^{j_{3}-j_{4}}\left(q \cdot \Delta-p_{1} \cdot \Delta-p_{5} \cdot \Delta-p_{6} \cdot \Delta\right)^{j_{2}-j_{3}} \\
& \left(q \cdot \Delta-p_{1} \cdot \Delta-p_{4} \cdot \Delta-p_{5} \cdot \Delta-p_{6} \cdot \Delta\right)^{j_{1}-j_{2}} \\
& +t^{a_{6}} t^{a_{3}} t^{a_{4}} t^{a_{5}}\left(q \cdot \Delta-p_{1} \cdot \Delta-p_{6} \cdot \Delta\right)^{j_{3}-j_{4}}\left(q \cdot \Delta-p_{1} \cdot \Delta-p_{3} \cdot \Delta-p_{6} \cdot \Delta\right)^{j_{2}-j_{3}} \\
& \left(q \cdot \Delta-p_{1} \cdot \Delta-p_{3} \cdot \Delta-p_{4} \cdot \Delta-p_{6} \cdot \Delta\right)^{j_{1}-j_{2}} \\
& +t^{a_{6}} t^{a_{3}} t^{a_{5}} t^{a_{4}}\left(q \cdot \Delta-p_{1} \cdot \Delta-p_{6} \cdot \Delta\right)^{j_{3}-j_{4}}\left(q \cdot \Delta-p_{1} \cdot \Delta-p_{3} \cdot \Delta-p_{6} \cdot \Delta\right)^{j_{2}-j_{3}} \\
& \left(q \cdot \Delta-p_{1} \cdot \Delta-p_{3} \cdot \Delta-p_{5} \cdot \Delta-p_{6} \cdot \Delta\right)^{j_{1}-j_{2}} \\
& +t^{a_{6}} t^{a_{4}} t^{a_{3}} t^{a_{5}}\left(q \cdot \Delta-p_{1} \cdot \Delta-p_{6} \cdot \Delta\right)^{j_{3}-j_{4}}\left(q \cdot \Delta-p_{1} \cdot \Delta-p_{4} \cdot \Delta-p_{6} \cdot \Delta\right)^{j_{2}-j_{3}} \\
& \left(q \cdot \Delta-p_{1} \cdot \Delta-p_{3} \cdot \Delta-p_{4} \cdot \Delta-p_{6} \cdot \Delta\right)^{j_{1}-j_{2}} \\
& +t^{a_{6}} t^{a_{4}} t^{a_{5}} t^{a_{3}}\left(q \cdot \Delta-p_{1} \cdot \Delta-p_{6} \cdot \Delta\right)^{j_{3}-j_{4}}\left(q \cdot \Delta-p_{1} \cdot \Delta-p_{4} \cdot \Delta-p_{6} \cdot \Delta\right)^{j_{2}-j_{3}} \\
& \left(q \cdot \Delta-p_{1} \cdot \Delta-p_{4} \cdot \Delta-p_{5} \cdot \Delta-p_{6} \cdot \Delta\right)^{j_{1}-j_{2}}
\end{aligned}
$$




$$
\begin{aligned}
& +t^{a_{6}} t^{a_{5}} t^{a_{3}} t^{a_{4}}\left(q \cdot \Delta-p_{1} \cdot \Delta-p_{6} \cdot \Delta\right)^{j_{3}-j_{4}}\left(q \cdot \Delta-p_{1} \cdot \Delta-p_{5} \cdot \Delta-p_{6} \cdot \Delta\right)^{j_{2}-j_{3}} \\
& \quad\left(q \cdot \Delta-p_{1} \cdot \Delta-p_{3} \cdot \Delta-p_{5} \cdot \Delta-p_{6} \cdot \Delta\right)^{j_{1}-j_{2}} \\
& +t^{a_{6}} t^{a_{5}} t^{a_{4}} t^{a_{3}}\left(q \cdot \Delta-p_{1} \cdot \Delta-p_{6} \cdot \Delta\right)^{j_{3}-j_{4}}\left(q \cdot \Delta-p_{1} \cdot \Delta-p_{5} \cdot \Delta-p_{6} \cdot \Delta\right)^{j_{2}-j_{3}} \\
& \left.\quad\left(q \cdot \Delta-p_{1} \cdot \Delta-p_{4} \cdot \Delta-p_{5} \cdot \Delta-p_{6} \cdot \Delta\right)^{j_{1}-j_{2}}\right)
\end{aligned}
$$

\section{B Mellin moments at four loops}

Here we present the anomalous dimensions at four loops for $1 \leq N \leq 16$. Obviously, $\gamma_{\text {ns }}^{-}(N=1)=0$ at all orders. To fix our normalization, we write down the complete expression for $N=2$ including all lower orders. Recall that $a_{\mathrm{s}}=\alpha_{\mathrm{s}} /(4 \pi)$.

$$
\begin{aligned}
\gamma_{\mathrm{ns}}^{+}(N=2)= & \left\{\frac{8}{3} C_{F}\right\}+a_{\mathrm{s}}^{2}\left\{-\frac{112}{27} C_{F}^{2}+\frac{376}{27} C_{A} C_{F}-\frac{64}{27} n_{f} C_{F}\right\} \\
a_{\mathrm{s}} & a_{\mathrm{s}}^{3}\left\{C_{F}^{3}\left[-\frac{560}{243}+\frac{128}{3} \zeta_{3}\right]+C_{A} C_{F}^{2}\left[-\frac{8528}{243}-64 \zeta_{3}\right]+C_{A}^{2} C_{F}\left[\frac{20920}{243}+\frac{64}{3} \zeta_{3}\right]\right. \\
& \left.+n_{f} C_{F}^{2}\left[-\frac{3412}{243}+\frac{64}{3} \zeta_{3}\right]+n_{f} C_{A} C_{F}\left[-\frac{3128}{243}-\frac{64}{3} \zeta_{3}\right]+n_{f}^{2} C_{F}\left[-\frac{224}{243}\right]\right\} \\
+ & a_{\mathrm{s}}^{4}\left\{C_{F}^{4}\left[\frac{194392}{2187}+\frac{10880}{81} \zeta_{3}-\frac{1280}{3} \zeta_{5}\right]+C_{A} C_{F}^{3}\left[\frac{238676}{2187}+\frac{31040}{81} \zeta_{3}-\frac{704}{3} \zeta_{4}+\frac{1280}{3} \zeta_{5}\right]\right. \\
& +C_{A}^{2} C_{F}^{2}\left[-\frac{1626064}{2187}-\frac{25744}{27} \zeta_{3}+352 \zeta_{4}+\frac{4480}{9} \zeta_{5}\right] \\
& +C_{A}^{3} C_{F}\left[\frac{1734130}{2187}+\frac{34936}{81} \zeta_{3}-\frac{352}{3} \zeta_{4}-\frac{12160}{27} \zeta_{5}\right] \\
& +n_{f} C_{F}^{3}\left[\frac{190912}{2187}-\frac{2528}{81} \zeta_{3}+\frac{128}{3} \zeta_{4}-\frac{640}{3} \zeta_{5}\right] \\
& +n_{f} C_{A} C_{F}^{2}\left[-\frac{177748}{2187}+\frac{12928}{27} \zeta_{3}-\frac{544}{3} \zeta_{4}+\frac{320}{9} \zeta_{5}\right] \\
& +n_{f} C_{A}^{2} C_{F}\left[-\frac{53018}{243}-\frac{4040}{9} \zeta_{3}+\frac{416}{3} \zeta_{4}+\frac{4480}{27} \zeta_{5}\right]+n_{f}^{2} C_{F}^{2}\left[\frac{24944}{2187}-\frac{128}{3} \zeta_{3}+\frac{64}{3} \zeta_{4}\right] \\
& +n_{f}^{2} C_{A} C_{F}\left[\frac{6350}{729}+\frac{128}{3} \zeta_{3}-\frac{64}{3} \zeta_{4}\right]+n_{f}^{3} C_{F}\left[-\frac{1024}{2187}+\frac{128}{81} \zeta_{3}\right] \\
N_{R} & {\left.\left[-\frac{736}{9}+\frac{1984}{9} \zeta_{3}+\frac{5120}{9} \zeta_{5}\right]+n_{f} \frac{d_{F}^{a b c d} d_{F}^{a b c d}}{N_{R}}\left[\frac{832}{9}+\frac{1024}{9} \zeta_{3}-\frac{2560}{9} \zeta_{5}\right]\right\} }
\end{aligned}
$$


The four-loop coefficient for the even moments $N=4$ to $N=16$ are given by

$$
\begin{aligned}
& \gamma_{\mathrm{ns}}^{(3)+}(N=4)= \\
& C_{F}^{4}\left[\frac{3482407012657}{34992000000}+\frac{14504764}{50625} \zeta_{3}-\frac{25136}{45} \zeta_{5}\right] \\
& +C_{A} C_{F}^{3}\left[\frac{33802068299}{174960000}-\frac{10215349}{81000} \zeta_{3}-\frac{15829}{75} \zeta_{4}+\frac{33004}{45} \zeta_{5}\right] \\
& +C_{A}^{2} C_{F}^{2}\left[-\frac{1557367902137}{1749600000}-\frac{2497339}{16875} \zeta_{3}+\frac{15829}{50} \zeta_{4}-\frac{1645}{9} \zeta_{5}\right] \\
& +C_{A}^{3} C_{F}\left[\frac{49455970561}{43740000}+\frac{13461191}{81000} \zeta_{3}-\frac{15829}{150} \zeta_{4}-\frac{18646}{135} \zeta_{5}\right] \\
& +n_{f} C_{F}^{3}\left[\frac{29581840417}{174960000}+\frac{820961}{10125} \zeta_{3}+\frac{2878}{75} \zeta_{4}-\frac{1256}{3} \zeta_{5}\right] \\
& +n_{f} C_{A} C_{F}^{2}\left[-\frac{89325051233}{437400000}+\frac{4588639}{6750} \zeta_{3}-\frac{21587}{75} \zeta_{4}+\frac{628}{9} \zeta_{5}\right] \\
& +n_{f} C_{A}^{2} C_{F}\left[-\frac{1796654459}{4860000}-\frac{5247961}{6750} \zeta_{3}+\frac{18709}{75} \zeta_{4}+\frac{43736}{135} \zeta_{5}\right] \\
& +n_{f}^{2} C_{F}^{2}\left[\frac{5419760639}{218700000}-\frac{2146}{25} \zeta_{3}+\frac{628}{15} \zeta_{4}\right]+n_{f}^{2} C_{A} C_{F}\left[\frac{60167591}{3645000}+\frac{2146}{25} \zeta_{3}-\frac{628}{15} \zeta_{4}\right] \\
& +n_{f}^{3} C_{F}\left[-\frac{17813699}{21870000}+\frac{1256}{405} \zeta_{3}\right]+\frac{d_{F}^{a b c d} d_{A}^{a b c d}}{N_{R}}\left[\frac{254713}{1350}+\frac{63568}{45} \zeta_{3}-\frac{78868}{45} \zeta_{5}\right] \\
& +n_{f} \frac{d_{F}^{a b c d} d_{F}^{a b c d}}{N_{R}}\left[\frac{16568}{135}+\frac{22552}{75} \zeta_{3}-\frac{26912}{45} \zeta_{5}\right], \\
& \gamma_{\mathrm{ns}}^{(3)+}(N=6)= \\
& C_{F}^{4}\left[\frac{287471623549488131}{1801088541000000}+\frac{33026498018}{121550625} \zeta_{3}-\frac{450712}{735} \zeta_{5}\right] \\
& +C_{A} C_{F}^{3}\left[\frac{9809771626657}{476478450000}-\frac{9025033804}{121550625} \zeta_{3}-\frac{768482}{3675} \zeta_{4}+\frac{621976}{735} \zeta_{5}\right] \\
& +C_{A}^{2} C_{F}^{2}\left[-\frac{588570119595401}{918922725000}+\frac{3466052671}{27011250} \zeta_{3}+\frac{384241}{1225} \zeta_{4}-\frac{1695382}{2205} \zeta_{5}\right] \\
& +C_{A}^{3} C_{F}\left[\frac{64997866579309}{56010528000}+\frac{395253829}{27783000} \zeta_{3}-\frac{384241}{3675} \zeta_{4}+\frac{1378042}{6615} \zeta_{5}\right] \\
& +n_{f} C_{F}^{3}\left[\frac{2697261071752787}{12864918150000}+\frac{2830802}{19845} \zeta_{3}+\frac{139724}{3675} \zeta_{4}-\frac{11344}{21} \zeta_{5}\right] \\
& +n_{f} C_{A} C_{F}^{2}\left[-\frac{160989027584717}{612615150000}+\frac{8830621}{11025} \zeta_{3}-\frac{1301446}{3675} \zeta_{4}+\frac{5672}{63} \zeta_{5}\right]
\end{aligned}
$$




$$
\begin{aligned}
& +n_{f} C_{A}^{2} C_{F}\left[-\frac{96176330975533}{210039480000}-\frac{1122625478}{1157625} \zeta_{3}+\frac{1161722}{3675} \zeta_{4}+\frac{548152}{1323} \zeta_{5}\right] \\
& +n_{f}^{2} C_{F}^{2}\left[\frac{5177594850433}{153153787500}-\frac{3697676}{33075} \zeta_{3}+\frac{5672}{105} \zeta_{4}\right] \\
& +n_{f}^{2} C_{A} C_{F}\left[\frac{348632126303}{17503290000}+\frac{3697676}{33075} \zeta_{3}-\frac{5672}{105} \zeta_{4}\right]+n_{f}^{3} C_{F}\left[-\frac{26381269339}{26254935000}+\frac{11344}{2835} \zeta_{3}\right] \\
& +\frac{d_{F}^{a b c d} d_{A}^{a b c d}}{N_{R}}\left[\frac{1816777297}{2646000}+\frac{48954263}{18375} \zeta_{3}-\frac{8666912}{2205} \zeta_{5}\right] \\
& +n_{f} \frac{d_{F}^{a b c d} d_{F}^{a b c d}}{N_{R}}\left[\frac{70029019}{330750}+\frac{4621504}{11025} \zeta_{3}-\frac{379264}{441} \zeta_{5}\right], \\
& \gamma_{\mathrm{ns}}^{(3)+}(N=8)= \\
& C_{F}^{4}\left[\frac{1526099947627950150458381}{8067032349014016000000}+\frac{20349260276089}{78764805000} \zeta_{3}-\frac{1804282}{2835} \zeta_{5}\right] \\
& +C_{A} C_{F}^{3}\left[-\frac{1421752209961884815677}{19207219878604800000}-\frac{3387289715833}{140026320000} \zeta_{3}-\frac{27614477}{132300} \zeta_{4}+\frac{17728727}{19845} \zeta_{5}\right] \\
& +C_{A}^{2} C_{F}^{2}\left[-\frac{136950111821419605151}{304876506009600000}+\frac{81027197994671}{210039480000} \zeta_{3}+\frac{27614477}{88200} \zeta_{4}-\frac{4834637}{3780} \zeta_{5}\right] \\
& +C_{A}^{3} C_{F}\left[\frac{1008933995789394973}{871075731456000}-\frac{33568622483}{244944000} \zeta_{3}-\frac{27614477}{264600} \zeta_{4}+\frac{31576003}{59535} \zeta_{5}\right] \\
& +n_{f} C_{F}^{3}\left[\frac{1136310990563809311301}{4801804969651200000}+\frac{5612836699}{30005640} \zeta_{3}+\frac{2510407}{66150} \zeta_{4}-\frac{39532}{63} \zeta_{5}\right] \\
& +n_{f} C_{A} C_{F}^{2}\left[-\frac{11533221344476533811}{38109563251200000}+\frac{88755158137}{100018800} \zeta_{3}-\frac{17730227}{44100} \zeta_{4}+\frac{19766}{189} \zeta_{5}\right] \\
& +n_{f} C_{A}^{2} C_{F}\left[-\frac{189224450730372949}{362948221440000}-\frac{1661823807613}{1500282000} \zeta_{3}+\frac{48169867}{132300} \zeta_{4}+\frac{813749}{1701} \zeta_{5}\right] \\
& +n_{f}^{2} C_{F}^{2}\left[\frac{386628670506434189}{9527390812800000}-\frac{77750543}{595350} \zeta_{3}+\frac{19766}{315} \zeta_{4}\right] \\
& +n_{f}^{2} C_{A} C_{F}\left[\frac{2001327622434827}{90737055360000}+\frac{77750543}{595350} \zeta_{3}-\frac{19766}{315} \zeta_{4}\right] \\
& +n_{f}^{3} C_{F}\left[-\frac{77182042631063}{68052791520000}+\frac{39532}{8505} \zeta_{3}\right] \\
& +\frac{d_{F}^{a b c d} d_{A}^{a b c d}}{N_{R}}\left[\frac{245446938529151}{216040608000}+\frac{964237792849}{250047000} \zeta_{3}-\frac{117093083}{19845} \zeta_{5}\right] \\
& +n_{f} \frac{d_{F}^{a b c d} d_{F}^{a b c d}}{N_{R}}\left[\frac{1164731326841}{3857868000}+\frac{892046473}{1786050} \zeta_{3}-\frac{605768}{567} \zeta_{5}\right] \text {, }
\end{aligned}
$$




$$
\begin{aligned}
& \gamma_{\mathrm{ns}}^{(3)+}(N=10)= \\
& C_{F}^{4}\left[\frac{2017383724760695233171991134991}{9825227427985488199296000000}+\frac{143667693462054187}{576597755002500} \zeta_{3}\right. \\
& \left.-\frac{222343766}{343035} \zeta_{5}\right] \\
& +C_{A} C_{F}^{3}\left[-\frac{1244204474536625986344420833}{9666693652091192640000000}+\frac{8470409428966531}{768797006670000} \zeta_{3}\right. \\
& \left.-\frac{151796299}{727650} \zeta_{4}+\frac{440406986}{480249} \zeta_{5}\right] \\
& +C_{A}^{2} C_{F}^{2}\left[-\frac{8344774766025843618923173}{27898105778040960000000}+\frac{488048876510787149}{768797006670000} \zeta_{3}\right. \\
& \left.+\frac{151796299}{485100} \zeta_{4}-\frac{79369519}{45738} \zeta_{5}\right] \\
& +C_{A}^{3} C_{F}\left[\frac{37809477198339250105213}{32937551095680000000}-\frac{1032212221214159}{3630682440000} \zeta_{3}-\frac{151796299}{1455300} \zeta_{4}\right. \\
& \left.+\frac{2378996101}{2881494} \zeta_{5}\right] \\
& +n_{f} C_{F}^{3}\left[\frac{273095498113782515546834719}{1063336301730031190400000}+\frac{110938456177789}{499218835500} \zeta_{3}\right. \\
& \left.+\frac{151796299}{4002075} \zeta_{4}-\frac{482200}{693} \zeta_{5}\right] \\
& +n_{f} C_{A} C_{F}^{2}\left[-\frac{232521423145307204017937}{697452644451024000000}+\frac{317697741600923}{332812557000} \zeta_{3}\right. \\
& \left.-\frac{1172854799}{2668050} \zeta_{4}+\frac{241100}{2079} \zeta_{5}\right] \\
& +n_{f} C_{A}^{2} C_{F}\left[-\frac{3449950935218411700067}{6038551034208000000}-\frac{168584409069107}{138671898750} \zeta_{3}\right. \\
& \left.+\frac{3214971799}{8004150} \zeta_{4}+\frac{181204484}{343035} \zeta_{5}\right] \\
& +n_{f}^{2} C_{F}^{2}\left[\frac{17653381355656128339793}{383598954448063200000}-\frac{5236700507}{36018675} \zeta_{3}+\frac{48220}{693} \zeta_{4}\right] \\
& +n_{f}^{2} C_{A} C_{F}\left[\frac{7133251712396708693}{301927551710400000}+\frac{5236700507}{36018675} \zeta_{3}-\frac{48220}{693} \zeta_{4}\right] \\
& +n_{f}^{3} C_{F}\left[-\frac{613551152411968391}{498180460322160000}+\frac{96440}{18711} \zeta_{3}\right]
\end{aligned}
$$




$$
\begin{aligned}
& +\frac{d_{F}^{a b c d} d_{A}^{a b c d}}{N_{R}}\left[\frac{6387465101013091}{4149351360000}+\frac{751245802206203}{151278435000} \zeta_{3}-\frac{18465293144}{2401245} \zeta_{5}\right] \\
& +n_{f} \frac{d_{F}^{a b c d} d_{F}^{a b c d}}{N_{R}}\left[\frac{51618947156153}{134469720000}+\frac{18626595374}{33350625} \zeta_{3}-\frac{141624128}{114345} \zeta_{5}\right], \\
& \gamma_{\mathrm{ns}}^{(3)+}(N=12)= \\
& C_{F}^{4}\left[\frac{331516931678819616019516119238120540753}{1541296125734534205067061110080000000}+\frac{3996930795976177310551}{16468208480626402500} \zeta_{3}\right. \\
& \left.-\frac{37964613926}{57972915} \zeta_{5}\right] \\
& +C_{A} C_{F}^{3}\left[-\frac{41810347217755725651032771751930697}{256626061560861506005171680000000}+\frac{98597799463449331819}{2744701413437733750} \zeta_{3}\right. \\
& \left.-\frac{25648239313}{122972850} \zeta_{4}+\frac{34328650826}{36891855} \zeta_{5}\right] \\
& +C_{A}^{2} C_{F}^{2}\left[-\frac{238851091715148666377698033403459}{1367305023301293405288960000000}+\frac{38330693033675619062839}{43915222615003740000} \zeta_{3}\right. \\
& \left.+\frac{25648239313}{81981900} \zeta_{4}-\frac{27699141079}{12882870} \zeta_{5}\right] \\
& +C_{A}^{3} C_{F}\left[\frac{516560961693213572859380630401}{455313028072358776320000000}-\frac{3552780661279687493}{8356447859760000} \zeta_{3}\right. \\
& \left.-\frac{25648239313}{245945700} \zeta_{4}+\frac{242831514391}{221351130} \zeta_{5}\right] \\
& +n_{f} C_{F}^{3}\left[\frac{14007724721120701350168397616773189}{51325212312172301201034336000000}+\frac{275829519782497567}{1096783781593500} \zeta_{3}\right. \\
& \left.+\frac{25648239313}{676350675} \zeta_{4}-\frac{6774784}{9009} \zeta_{5}\right] \\
& +n_{f} C_{A} C_{F}^{2}\left[-\frac{12260374152873668292904144517329}{34182625582532335132224000000}+\frac{246056489027368057}{243729729243000} \zeta_{3}\right. \\
& \left.-\frac{212141105873}{450900450} \zeta_{4}+\frac{3387392}{27027} \zeta_{5}\right] \\
& +n_{f} C_{A}^{2} C_{F}\left[-\frac{581173376177155203686777699}{948568808484080784000000}-\frac{119245993023506038}{91398648466125} \zeta_{3}\right. \\
& \left.+\frac{585126838993}{1352701350} \zeta_{4}+\frac{599861228}{1054053} \zeta_{5}\right] \\
& +n_{f}^{2} C_{F}^{2}\left[\frac{14406272473786523198816959409}{284855213187769459435200000}-\frac{191866603189}{1217431215} \zeta_{3}+\frac{3387392}{45045} \zeta_{4}\right]
\end{aligned}
$$




$$
\begin{aligned}
& +n_{f}^{2} C_{A} C_{F}\left[\frac{442446968888889719821403}{17785665159076514700000}+\frac{191866603189}{1217431215} \zeta_{3}-\frac{3387392}{45045} \zeta_{4}\right] \\
& +n_{f}^{3} C_{F}\left[-\frac{18633947419926787652303}{14228532127261211760000}+\frac{6774784}{1216215} \zeta_{3}\right] \\
& +\frac{d_{F}^{a b c d} d_{A}^{a b c d}}{N_{R}}\left[\frac{55568956318895924814773}{29157574993747200000}+\frac{224835066938602217}{37496881422000} \zeta_{3}-\frac{3785627672012}{405810405} \zeta_{5}\right] \\
& +n_{f} \frac{d_{F}^{a b c d} d_{F}^{a b c d}}{N_{R}}\left[\frac{22664035135268075399}{49587712574400000}+\frac{18402607883189}{30435780375} \zeta_{3}-\frac{485441696}{351351} \zeta_{5}\right], \\
& \gamma_{\mathrm{ns}}^{(3)+}(N=14)= \\
& C_{F}^{4}\left[\frac{97523736280058513278817419051773381749}{440370321638438344304874602880000000}+\frac{1567683874499713548181}{6587283392250561000} \zeta_{3}\right. \\
& \left.-\frac{267445675058}{405810405} \zeta_{5}\right] \\
& +C_{A} C_{F}^{3}\left[-\frac{333627771877952197537388891905440907}{1796382430926030542036201760000000}+\frac{118827371645736248177}{2195761130750187000} \zeta_{3}\right. \\
& \left.-\frac{3663695353}{17567550} \zeta_{4}+\frac{6927566278}{7378371} \zeta_{5}\right] \\
& +C_{A}^{2} C_{F}^{2}\left[-\frac{86656353251877447263713613209043}{1288422041187757247291520000000}+\frac{6031813059800336882399}{5489402826875467500} \zeta_{3}\right. \\
& \left.+\frac{3663695353}{11711700} \zeta_{4}-\frac{45655020935}{18036018} \zeta_{5}\right] \\
& +C_{A}^{3} C_{F}\left[\frac{3974468919372874040522232093269}{3549372014291342279040000000}-\frac{8803366113150246019}{15748690197240000} \zeta_{3}\right. \\
& \left.-\frac{3663695353}{35135100} \zeta_{4}+\frac{59678850515}{44270226} \zeta_{5}\right] \\
& +n_{f} C_{F}^{3}\left[\frac{1469298768736788933360199563898027}{5132521231217230120103433600000}+\frac{303399887420914357}{1096783781593500} \zeta_{3}\right. \\
& \left.+\frac{3663695353}{96621525} \zeta_{4}-\frac{7204840}{9009} \zeta_{5}\right] \\
& +n_{f} C_{A} C_{F}^{2}\left[-\frac{22747053762883618602535741424929}{59819594769431586481392000000}+\frac{257384193913101469}{243729729243000} \zeta_{3}\right. \\
& \left.-\frac{31996728653}{64414350} \zeta_{4}+\frac{3602420}{27027} \zeta_{5}\right] \\
& +n_{f} C_{A}^{2} C_{F}\left[-\frac{15060401544121460041592815421}{23239935807859979208000000}-\frac{17663095878365457683}{12795810785257500} \zeta_{3}\right.
\end{aligned}
$$




$$
\begin{aligned}
& \left.+\frac{88662795253}{193243050} \zeta_{4}+\frac{131795548}{218295} \zeta_{5}\right] \\
& +n_{f}^{2} C_{F}^{2}\left[\frac{776154252048358965658599007}{14242760659388472971760000}-\frac{1022665028447}{6087156075} \zeta_{3}+\frac{720484}{9009} \zeta_{4}\right] \\
& +n_{f}^{2} C_{A} C_{F}\left[\frac{10327818039687232051823761}{398398899563313929280000}+\frac{1022665028447}{6087156075} \zeta_{3}-\frac{720484}{9009} \zeta_{4}\right] \\
& +n_{f}^{3} C_{F}\left[-\frac{3911903561688643214011}{2845706425452242352000}+\frac{1440968}{243243} \zeta_{3}\right] \\
& +\frac{d_{F}^{a b c d} d_{A}^{a b c d}}{N_{R}}\left[\frac{1062557701742240655559903}{473810593648392000000}+\frac{59328382591268105459}{8530540523505000} \zeta_{3}\right. \\
& \left.-\frac{4399336453016}{405810405} \zeta_{5}\right] \\
& +n_{f} \frac{d_{F}^{a b c d} d_{F}^{a b c d}}{N_{R}}\left[\frac{144413233152647568760141}{276389512961562000000}+\frac{4789034376385918}{7456766191875} \zeta_{3}-\frac{1423427008}{945945} \zeta_{5}\right] \text {, } \\
& \gamma_{\mathrm{ns}}^{(3)+}(N=16)= \\
& C_{F}^{4}\left[\frac{585091838928074604746949501506640014143607792143679}{2590529154801458855855422389944869371248640000000}\right. \\
& \left.+\frac{10317600287587322672417525639}{44014119696332728422480000} \zeta_{3}-\frac{22176170947759}{33508344870} \zeta_{5}\right] \\
& +C_{A} C_{F}^{3}\left[-\frac{1377628380634900156963463567256914633249848729}{6830915722139930955540672272529162240000000}\right. \\
& \left.+\frac{15923437134502520764317446141}{234741971713774551586560000} \zeta_{3}-\frac{59290512768143}{284313229200} \zeta_{4}+\frac{40278295293893}{42646984380} \zeta_{5}\right] \\
& +C_{A}^{2} C_{F}^{2}\left[\frac{258914251298466366542816679113637834371}{9203567921633206758178924462080000000}\right. \\
& \left.+\frac{22014640304042236864687668923}{16767283693841039399040000} \zeta_{3}+\frac{59290512768143}{189542152800} \zeta_{4}-\frac{1804393628665651}{625489104240} \zeta_{5}\right] \\
& +C_{A}^{3} C_{F}\left[\frac{14183627547279601657840082208324742517}{12843000517166864476020965376000000}\right. \\
& \left.-\frac{2649527312649305104376683}{3862475881756882790400} \zeta_{3}-\frac{59290512768143}{568626458400} \zeta_{4}+\frac{101176031536771}{63970476570} \zeta_{5}\right] \\
& +n_{f} C_{F}^{3}\left[\frac{94403012063925170595517180859037562017787057}{317149658527925365792959784081711104000000}\right.
\end{aligned}
$$




$$
\begin{aligned}
& \left.+\frac{25752552164686212840197}{86215979503501848000} \zeta_{3}+\frac{59290512768143}{1563722760600} \zeta_{4}-\frac{128839202}{153153} \zeta_{5}\right] \\
+ & n_{f} C_{A} C_{F}^{2}\left[-\frac{2893023190248781306760837247560962327027}{724780973828615032206590301388800000}\right. \\
& \left.+\frac{63010566480259446207079}{57477319669001232000} \zeta_{3}-\frac{541630986863623}{1042481840400} \zeta_{4}+\frac{64419601}{459459} \zeta_{5}\right] \\
+ & n_{f} C_{A}^{2} C_{F}\left[-\frac{674736712195588046827798227902824541}{993803611447435941596860416000000}-\frac{11083840592301151093813}{7663642622533497600} \zeta_{3}\right. \\
& \left.+\frac{1506311935054583}{3127445521200} \zeta_{4}+\frac{4569981743}{7209972} \zeta_{5}\right] \\
+ & n_{f}^{2} C_{F}^{2}\left[\frac{1499767303942721479649857809345185131}{25885034779593394007378225049600000}-\frac{2491999394100703}{14073504845400} \zeta_{3}\right. \\
& \left.+\frac{64419601}{765765} \zeta_{4}\right] \\
+ & n_{f}^{2} C_{A} C_{F}\left[\frac{95217199819008483482793882523327}{3549298612312271219988787200000}+\frac{2491999394100703}{14073504845400} \zeta_{3}-\frac{64419601}{765765} \zeta_{4}\right] \\
+ & n_{f} \frac{d_{F}^{a b c d} d_{F}^{a b c d}}{N_{R}}\left[\frac{63048309341247319711503473}{108438494006543500800000}+\frac{1361755723179569}{2020810952160} \zeta_{3}\right. \\
+ & n_{f}^{3} C_{F}\left[-\frac{54396936195548207942967542719}{38028199417631477357022720000}+\frac{128839202}{20675655} \zeta_{3}\right] \\
+ & \frac{d_{F}^{a b c d} d_{A}^{a b c d}}{N_{R}}\left[\frac{13544672049501491403276515417}{5301437484764348928000000}+\frac{3791955378871486958507}{483002686294128000} \zeta_{3}\right. \\
& \left.\frac{5744913623184647}{469116828180} \zeta_{5}\right]
\end{aligned}
$$

The corresponding results for the odd moments $N=3$ to $N=15$ read

$$
\begin{aligned}
& \gamma_{\mathrm{ns}}^{(3)-}(N=3)= \\
& C_{F}^{4}\left[\frac{341611945}{2239488}+\frac{24380}{81} \zeta_{3}-\frac{2000}{3} \zeta_{5}\right] \\
& +C_{F}^{3} C_{A}\left[\frac{40709323}{279936}-\frac{140057}{648} \zeta_{3}-\frac{605}{3} \zeta_{4}+\frac{2900}{3} \zeta_{5}\right] \\
& +C_{F}^{2} C_{A}^{2}\left[-\frac{503877829}{559872}-\frac{4843}{27} \zeta_{3}+\frac{605}{2} \zeta_{4}-\frac{1325}{9} \zeta_{5}\right]
\end{aligned}
$$




$$
\begin{aligned}
& +C_{F} C_{A}^{3}\left[\frac{72667541}{69984}+\frac{125219}{648} \zeta_{3}-\frac{605}{6} \zeta_{4}-\frac{5950}{27} \zeta_{5}\right] \\
& +C_{F}^{3} n_{f}\left[\frac{38386673}{279936}+\frac{3493}{81} \zeta_{3}+\frac{110}{3} \zeta_{4}-\frac{1000}{3} \zeta_{5}\right] \\
& +C_{F}^{2} C_{A} n_{f}\left[-\frac{22941613}{139968}+\frac{31547}{54} \zeta_{3}-\frac{715}{3} \zeta_{4}+\frac{500}{9} \zeta_{5}\right] \\
& +C_{F} C_{A}^{2} n_{f}\left[-\frac{2366971}{7776}-\frac{11483}{18} \zeta_{3}+\frac{605}{3} \zeta_{4}+\frac{7000}{27} \zeta_{5}\right] \\
& +C_{F}^{2} n_{f}^{2}\left[\frac{1313443}{69984}-\frac{610}{9} \zeta_{3}+\frac{100}{3} \zeta_{4}\right]+C_{F} C_{A} n_{f}^{2}\left[\frac{79747}{5832}+\frac{610}{9} \zeta_{3}-\frac{100}{3} \zeta_{4}\right] \\
& +C_{F} n_{f}^{3}\left[-\frac{23587}{34992}+\frac{200}{81} \zeta_{3}\right]+\frac{d_{F}^{a b c d} d_{A}^{a b c d}}{N_{R}}\left[-\frac{85}{3}+\frac{7600}{9} \zeta_{3}-\frac{7300}{9} \zeta_{5}\right] \\
& +n_{f} \frac{d_{F}^{a b c d} d_{F}^{a b c d}}{N_{R}}\left[\frac{275}{3}+\frac{1960}{9} \zeta_{3}-\frac{4000}{9} \zeta_{5}\right], \\
& \gamma_{\mathrm{ns}}^{(3)-}(N=5)= \\
& C_{F}^{4}\left[\frac{421526640437}{2187000000}+\frac{16940714}{50625} \zeta_{3}-\frac{11032}{15} \zeta_{5}\right] \\
& +C_{F}^{3} C_{A}\left[-\frac{27591809}{12150000}-\frac{2335808}{10125} \zeta_{3}-\frac{15554}{75} \zeta_{4}+\frac{16408}{15} \zeta_{5}\right] \\
& +C_{F}^{2} C_{A}^{2}\left[-\frac{151355831129}{218700000}+\frac{413533}{3750} \zeta_{3}+\frac{7777}{25} \zeta_{4}-\frac{6398}{9} \zeta_{5}\right] \\
& +C_{F} C_{A}^{3}\left[\frac{44149637147}{38880000}+\frac{4424851}{81000} \zeta_{3}-\frac{7777}{75} \zeta_{4}+\frac{13594}{135} \zeta_{5}\right] \\
& +n_{f} C_{F}^{3}\left[\frac{20671110851}{109350000}+\frac{1181666}{10125} \zeta_{3}+\frac{2828}{75} \zeta_{4}-\frac{1456}{3} \zeta_{5}\right] \\
& +n_{f} C_{F}^{2} C_{A}\left[-\frac{8570931889}{36450000}+\frac{92921}{125} \zeta_{3}-\frac{24262}{75} \zeta_{4}+\frac{728}{9} \zeta_{5}\right] \\
& +n_{f} C_{F} C_{A}^{2}\left[-\frac{36596231437}{87480000}-\frac{2976818}{3375} \zeta_{3}+\frac{21434}{75} \zeta_{4}+\frac{50456}{135} \zeta_{5}\right] \\
& +n_{f}^{2} C_{F}^{2}\left[\frac{269584781}{9112500}-\frac{67532}{675} \zeta_{3}+\frac{728}{15} \zeta_{4}\right]+n_{f}^{2} C_{F} C_{A}\left[\frac{44950469}{2430000}+\frac{67532}{675} \zeta_{3}-\frac{728}{15} \zeta_{4}\right] \\
& +n_{f}^{3} C_{F}\left[-\frac{10064827}{10935000}+\frac{1456}{405} \zeta_{3}\right]+\frac{d_{F}^{a b c d} d_{A}^{a b c d}}{N_{R}}\left[\frac{38339}{80}+\frac{52857}{25} \zeta_{3}-\frac{135968}{45} \zeta_{5}\right] \\
& +n_{f} \frac{d_{F}^{a b c d} d_{F}^{a b c d}}{N_{R}}\left[\frac{74501}{450}+\frac{82432}{225} \zeta_{3}-\frac{33152}{45} \zeta_{5}\right] \text {, }
\end{aligned}
$$




$$
\begin{aligned}
& \gamma_{\mathrm{ns}}^{(3)-}(N=7)= \\
& C_{F}^{4}\left[\frac{89260315086226821967}{409847703552000000}+\frac{107378934083}{324135000} \zeta_{3}-\frac{555362}{735} \zeta_{5}\right] \\
& +C_{A} C_{F}^{3}\left[-\frac{2731357455838412101}{26347352371200000}-\frac{976102618619}{5186160000} \zeta_{3}-\frac{1020151}{4900} \zeta_{4}+\frac{832501}{735} \zeta_{5}\right] \\
& +C_{A}^{2} C_{F}^{2}\left[-\frac{1842858791204823727}{3763907481600000}+\frac{964491706751}{2593080000} \zeta_{3}+\frac{3060453}{9800} \zeta_{4}-\frac{3607811}{2940} \zeta_{5}\right] \\
& +C_{A}^{3} C_{F}\left[\frac{6856528500444857}{5974456320000}-\frac{42180835897}{444528000} \zeta_{3}-\frac{1020151}{9800} \zeta_{4}+\frac{942569}{2205} \zeta_{5}\right] \\
& +n_{f} C_{F}^{3}\left[\frac{1462431578723251501}{6586838092800000}+\frac{44264401}{264600} \zeta_{3}+\frac{92741}{2450} \zeta_{4}-\frac{4108}{7} \zeta_{5}\right] \\
& +n_{f} C_{F}^{2} C_{A}\left[-\frac{132713980134736771}{470488435200000}+\frac{447543373}{529200} \zeta_{3}-\frac{1859803}{4900} \zeta_{4}+\frac{2054}{21} \zeta_{5}\right] \\
& +n_{f} C_{A}^{2} C_{F}\left[-\frac{6617534246169487}{13442526720000}-\frac{57952538719}{55566000} \zeta_{3}+\frac{1674321}{4900} \zeta_{4}+\frac{197849}{441} \zeta_{5}\right] \\
& +n_{f}^{2} C_{F}^{2}\left[\frac{1463487948290143}{39207369600000}-\frac{8059127}{66150} \zeta_{3}+\frac{2054}{35} \zeta_{4}\right] \\
& +n_{f}^{2} C_{F} C_{A}\left[\frac{23660663137019}{1120210560000}+\frac{8059127}{66150} \zeta_{3}-\frac{2054}{35} \zeta_{4}\right] \\
& +n_{f}^{3} C_{F}\left[-\frac{902896393223}{840157920000}+\frac{4108}{945} \zeta_{3}\right] \\
& +\frac{d_{F}^{a b c d} d_{A}^{a b c d}}{N_{R}}\left[\frac{40269598361}{42336000}+\frac{885141073}{264600} \zeta_{3}-\frac{3727489}{735} \zeta_{5}\right] \\
& +n_{f} \frac{d_{F}^{a b c d} d_{F}^{a b c d}}{N_{R}}\left[\frac{584326699}{2268000}+\frac{30610507}{66150} \zeta_{3}-\frac{142568}{147} \zeta_{5}\right], \\
& \gamma_{\mathrm{ns}}^{(3)-}(N=9)= \\
& C_{F}^{4}\left[\frac{117290741389735897476581}{504189521813376000000}+\frac{12756265517567}{39382402500} \zeta_{3}-\frac{2166406}{2835} \zeta_{5}\right] \\
& +C_{A} C_{F}^{3}\left[-\frac{1414388556845197343069}{8574651731520000000}-\frac{2630697602423}{17503290000} \zeta_{3}-\frac{13785409}{66150} \zeta_{4}+\frac{4562074}{3969} \zeta_{5}\right] \\
& +C_{A}^{2} C_{F}^{2}\left[-\frac{624559062347065092853}{1905478162560000000}+\frac{32816667064709}{52509870000} \zeta_{3}+\frac{13785409}{44100} \zeta_{4}-\frac{91553}{54} \zeta_{5}\right] \\
& +C_{A}^{3} C_{F}\left[\frac{44405787094076715779}{38887309440000000}-\frac{7312706287799}{30005640000} \zeta_{3}-\frac{13785409}{132300} \zeta_{4}+\frac{17432173}{23814} \zeta_{5}\right]
\end{aligned}
$$




$$
\begin{aligned}
& +n_{f} C_{F}^{3}\left[\frac{29494412200734623467}{120045124241280000}+\frac{77319514799}{375070500} \zeta_{3}+\frac{1253219}{33075} \zeta_{4}-\frac{41800}{63} \zeta_{5}\right] \\
& +n_{f} C_{A} C_{F}^{2}\left[-\frac{15104254419980130497}{47636954064000000}+\frac{230513485753}{250047000} \zeta_{3}-\frac{9299719}{22050} \zeta_{4}+\frac{20900}{189} \zeta_{5}\right] \\
& +n_{f} C_{A}^{2} C_{F}\left[-\frac{2486710179097323617}{4536852768000000}-\frac{1091690689753}{937676250} \zeta_{3}+\frac{25392719}{66150} \zeta_{4}+\frac{4292332}{8505} \zeta_{5}\right] \\
& +n_{f}^{2} C_{F}^{2}\left[\frac{103302930942446363}{2381847703200000}-\frac{41192947}{297675} \zeta_{3}+\frac{4180}{63} \zeta_{4}\right] \\
& +n_{f}^{2} C_{A} C_{F}\left[\frac{5199820982878583}{226842638400000}+\frac{41192947}{297675} \zeta_{3}-\frac{4180}{63} \zeta_{4}\right] \\
& +n_{f}^{3} C_{F}\left[-\frac{40350728956471}{34026395760000}+\frac{8360}{1701} \zeta_{3}\right] \\
& +\frac{d_{F}^{a b c d} d_{A}^{a b c d}}{N_{R}}\left[\frac{2967981583758917}{2160406080000}+\frac{5621430297923}{1250235000} \zeta_{3}-\frac{137680664}{19845} \zeta_{5}\right] \\
& +n_{f} \frac{d_{F}^{a b c d} d_{F}^{a b c d}}{N_{R}}\left[\frac{92904062646461}{270050760000}+\frac{11854602254}{22325625} \zeta_{3}-\frac{3281344}{2835} \zeta_{5}\right], \\
& \gamma_{\mathrm{ns}}^{(3)-}(N=11)= \\
& C_{F}^{4}\left[\frac{5942479928802007050870338007469}{24563068569963720498240000000}+\frac{182985306975827551}{576597755002500} \zeta_{3}-\frac{263657654}{343035} \zeta_{5}\right] \\
& +C_{A} C_{F}^{3}\left[-\frac{140643955245651718928769629}{690478118006513760000000}-\frac{11683631163531071}{96099625833750} \zeta_{3}-\frac{151689577}{727650} \zeta_{4}\right. \\
& \left.+\frac{2781069694}{2401245} \zeta_{5}\right] \\
& +C_{A}^{2} C_{F}^{2}\left[-\frac{65080732936319895430465693}{334777269336491520000000}+\frac{1334072255443149799}{1537594013340000} \zeta_{3}+\frac{151689577}{485100} \zeta_{4}\right. \\
& \left.-\frac{23116873}{10890} \zeta_{5}\right] \\
& +C_{A}^{3} C_{F}\left[\frac{1934103459065790006173}{1711041615360000000}-\frac{937411447527013}{2420454960000} \zeta_{3}-\frac{151689577}{1455300} \zeta_{4}\right. \\
& \left.+\frac{14593135349}{14407470} \zeta_{5}\right] \\
& +n_{f} C_{F}^{3}\left[\frac{2807323127469698463268574221}{10633363017300311904000000}+\frac{118758665137891}{499218835500} \zeta_{3}+\frac{151689577}{4002075} \zeta_{4}\right. \\
& \left.-\frac{502528}{693} \zeta_{5}\right]
\end{aligned}
$$




$$
\begin{aligned}
& +n_{f} C_{A} C_{F}^{2}\left[-\frac{2888600005383327025035503}{8369431733412288000000}+\frac{109012601223061}{110937519000} \zeta_{3}-\frac{1215792617}{2668050} \zeta_{4}\right. \\
& \left.+\frac{251264}{2079} \zeta_{5}\right] \\
& +n_{f} C_{A}^{2} C_{F}\left[-\frac{1791290424092116907479}{3019275517104000000}-\frac{52497666373939}{41601569625} \zeta_{3}+\frac{3343998697}{8004150} \zeta_{4}\right. \\
& \left.+\frac{37705252}{68607} \zeta_{5}\right] \\
& +n_{f}^{2} C_{F}^{2}\left[\frac{37084682543660792132933}{767197908896126400000}-\frac{1093228621}{7203735} \zeta_{3}+\frac{251264}{3465} \zeta_{4}\right] \\
& +n_{f}^{2} C_{A} C_{F}\left[\frac{31274848451808887}{1286623089675000}+\frac{1093228621}{7203735} \zeta_{3}-\frac{251264}{3465} \zeta_{4}\right] \\
& +n_{f}^{3} C_{F}\left[-\frac{633953354507891423}{498180460322160000}+\frac{502528}{93555} \zeta_{3}\right] \\
& +\frac{d_{F}^{a b c d} d_{A}^{a b c d}}{N_{R}}\left[\frac{27544072006307986519}{15684548140800000}+\frac{112240841713507}{20170458000} \zeta_{3}-\frac{20755381868}{2401245} \zeta_{5}\right] \\
& +n_{f} \frac{d_{F}^{a b c d} d_{F}^{a b c d}}{N_{R}}\left[\frac{550922957081846869}{1307045678400000}+\frac{104968283381}{180093375} \zeta_{3}-\frac{30026464}{22869} \zeta_{5}\right], \\
& \gamma_{\mathrm{ns}}^{(3)-}(N=13)= \\
& +C_{F}^{4}\left[\frac{109254370623053143691943402173119101653}{440370321638438344304874602880000000}+\frac{10279814538281025664097}{32936416961252805000} \zeta_{3}\right. \\
& \left.-\frac{312960229682}{405810405} \zeta_{5}\right] \\
& +C_{A} C_{F}^{3}\left[-\frac{137433149672175525416627043272450693}{598794143642010180678733920000000}-\frac{122111455215270203911}{1219867294861215000} \zeta_{3}\right. \\
& \left.-\frac{3662719609}{17567550} \zeta_{4}+\frac{42916930526}{36891855} \zeta_{5}\right] \\
& +C_{A}^{2} C_{F}^{2}\left[-\frac{448216384754306083916951583828253}{5583162178480281404929920000000}+\frac{6030472603025623852733}{5489402826875467500} \zeta_{3}\right. \\
& \left.+\frac{3662719609}{11711700} \zeta_{4}-\frac{680613539689}{270540270} \zeta_{5}\right] \\
& +C_{A}^{3} C_{F}\left[\frac{43589208948057316412721986370967}{39043092157204765069440000000}-\frac{29267635901342586703}{55836265244760000} \zeta_{3}\right. \\
& \left.-\frac{3662719609}{35135100} \zeta_{4}+\frac{281672586271}{221351130} \zeta_{5}\right]
\end{aligned}
$$




$$
\begin{aligned}
& +n_{f} C_{F}^{3}\left[\frac{7156992636760583450168754611387207}{25662606156086150600517168000000}+\frac{290443295483160277}{1096783781593500} \zeta_{3}\right. \\
& \left.+\frac{3662719609}{96621525} \zeta_{4}-\frac{6997864}{9009} \zeta_{5}\right] \\
& +n_{f} C_{A} C_{F}^{2}\left[-\frac{22054474323190222766265253462577}{59819594769431586481392000000}+\frac{755458878766886551}{731189187729000} \zeta_{3}\right. \\
& \left.-\frac{31181819789}{64414350} \zeta_{4}+\frac{3498932}{27027} \zeta_{5}\right] \\
& +n_{f} C_{A}^{2} C_{F}\left[-\frac{44016652745476286989105451999}{69719807423579937624000000}-\frac{10316777282946572197}{7677486471154500} \zeta_{3}\right. \\
& \left.+\frac{86220020149}{193243050} \zeta_{4}+\frac{4331692556}{7378371} \zeta_{5}\right] \\
& +n_{f}^{2} C_{F}^{2}\left[\frac{3742938630150542702886432311}{71213803296942364858800000}-\frac{198433044499}{1217431215} \zeta_{3}+\frac{3498932}{45045} \zeta_{4}\right] \\
& +n_{f}^{2} C_{A} C_{F}\left[\frac{50680931177006728293019381}{1991994497816569646400000}+\frac{198433044499}{1217431215} \zeta_{3}-\frac{3498932}{45045} \zeta_{4}\right] \\
& +n_{f}^{3} C_{F}\left[-\frac{19115924965400169467303}{14228532127261211760000}+\frac{6997864}{1216215} \zeta_{3}\right] \\
& +\frac{d_{F}^{a b c d} d_{A}^{a b c d}}{N_{R}}\left[\frac{107463452886020580200393}{51025756239057600000}+\frac{2581697503167399049}{393717254931000} \zeta_{3}\right. \\
& \left.-\frac{4144734743192}{405810405} \zeta_{5}\right] \\
& +n_{f} \frac{d_{F}^{a b c d} d_{F}^{a b c d}}{N_{R}}\left[\frac{6259112433726496309379}{12756439059764400000}+\frac{2793224880481058}{4474059715125} \zeta_{3}-\frac{3554796992}{2459457} \zeta_{5}\right] \text {, } \\
& \gamma_{\mathrm{ns}}^{(3)-}(N=15)= \\
& C_{F}^{4}\left[\frac{1593418359971838203424246724210060722526639}{6313148931008652103954682306887680000000}+\frac{259680235193827374263}{843172274208071808} \zeta_{3}\right. \\
& \left.-\frac{89608395343}{115945830} \zeta_{5}\right] \\
& +C_{A} C_{F}^{3}\left[-\frac{910674811575654880535423035274865836261}{3678991218536510550090141204480000000}-\frac{47105526692823873500839}{562114849472047872000} \zeta_{3}\right. \\
& \left.-\frac{205125530543}{983782800} \zeta_{4}+\frac{34430154145}{29513484} \zeta_{5}\right]
\end{aligned}
$$




$$
\begin{aligned}
& +C_{A}^{2} C_{F}^{2}\left[\frac{24794744696072980162780614700810631}{1225105300877958891138908160000000}+\frac{264518190199865408922107}{200755303382874240000} \zeta_{3}\right. \\
& \left.+\frac{205125530543}{655855200} \zeta_{4}-\frac{415521270781}{144288144} \zeta_{5}\right] \\
& +C_{A}^{3} C_{F}\left[\frac{11008793137870484173113743839424629}{9995031592244419857776640000000}-\frac{4285776141034052935649}{6551455122051840000} \zeta_{3}\right. \\
& \left.-\frac{205125530543}{1967565600} \zeta_{4}+\frac{67011783863}{44270226} \zeta_{5}\right] \\
& +n_{f} C_{F}^{3}\left[\frac{765704555141235451117809578252282141}{2627850870383221821492958003200000}+\frac{5058205287659325397}{17548540505496000} \zeta_{3}\right. \\
& \left.+\frac{205125530543}{5410805400} \zeta_{4}-\frac{7397890}{9009} \zeta_{5}\right] \\
& +n_{f} C_{A} C_{F}^{2}\left[-\frac{5957585274731644785588178652001289}{15313816260974486139236352000000}+\frac{4197787411208679709}{3899675667888000} \zeta_{3}\right. \\
& \left.-\frac{1834288866343}{3607203600} \zeta_{4}+\frac{3698945}{27027} \zeta_{5}\right] \\
& +n_{f} C_{A}^{2} C_{F}\left[-\frac{718538849608998968737940767607}{1081713375784028123136000000}-\frac{165455940798017850551}{116990270036640000} \zeta_{3}\right. \\
& \left.+\frac{5092615537943}{10821610800} \zeta_{4}+\frac{77250091}{124740} \zeta_{5}\right] \\
& +n_{f}^{2} C_{F}^{2}\left[\frac{205033222941376179044590449727}{3646146728803449080770560000}-\frac{8409151022911}{48697248600} \zeta_{3}+\frac{739789}{9009} \zeta_{4}\right] \\
& +n_{f}^{2} C_{A} C_{F}\left[\frac{673351343605311635893107289}{25497529572052091473920000}+\frac{8409151022911}{48697248600} \zeta_{3}-\frac{739789}{9009} \zeta_{4}\right] \\
& +n_{f}^{3} C_{F}\left[-\frac{1022483569869843897775}{728500844915774042112}+\frac{1479578}{243243} \zeta_{3}\right] \\
& +\frac{d_{F}^{a b c d} d_{A}^{a b c d}}{N_{R}}\left[\frac{328171179053663132153783483}{135079092880123392000000}+\frac{145906081836810245387}{19498378339440000} \zeta_{3}\right. \\
& \left.-\frac{18940495337639}{1623241620} \zeta_{5}\right] \\
& +n_{f} \frac{d_{F}^{a b c d} d_{F}^{a b c d}}{N_{R}}\left[\frac{34218686926433684318010149}{61911250903389888000000}+\frac{22453298164820123}{34088074020000} \zeta_{3}\right. \\
& \left.-\frac{210864634}{135135} \zeta_{5}\right]
\end{aligned}
$$


and

$$
\begin{aligned}
& \gamma_{\mathrm{ns}}^{(3) \mathrm{s}}(N=3)= \\
& n_{f} C_{F} \frac{d^{a b c} d_{a b c}}{N_{R}}\left[-\frac{72365}{216}+\frac{3200}{9} \zeta_{3}\right] \\
& +n_{f} C_{A} \frac{d^{a b c} d_{a b c}}{N_{R}}\left[\frac{60629}{648}-\frac{1010}{9} \zeta_{3}+\frac{200}{9} \zeta_{5}\right]+n_{f}^{2} \frac{d^{a b c} d_{a b c}}{N_{R}}\left[-\frac{1879}{324}\right], \\
& \gamma_{\mathrm{ns}}^{(3) \mathrm{s}}(N=5)= \\
& n_{f} C_{F} \frac{d^{a b c} d_{a b c}}{N_{R}}\left[-\frac{1110626839}{5467500}+\frac{133952}{675} \zeta_{3}\right] \\
& +n_{f} C_{A} \frac{d^{a b c} d_{a b c}}{N_{R}}\left[\frac{11071396}{273375}-\frac{48608}{675} \zeta_{3}+\frac{1792}{45} \zeta_{5}\right]+n_{f}^{2} \frac{d^{a b c} d_{a b c}}{N_{R}}\left[\frac{150143}{273375}\right],
\end{aligned}
$$

$\gamma_{\mathrm{ns}}^{(3) \mathrm{s}}(N=7)=$

$$
\begin{aligned}
& n_{f} C_{F} \frac{d^{a b c} d_{a b c}}{N_{R}}\left[-\frac{779352339134399}{5601052800000}+\frac{116051}{882} \zeta_{3}\right] \\
& +n_{f} C_{A} \frac{d^{a b c} d_{a b c}}{N_{R}}\left[\frac{4572181575853}{240045120000}-\frac{3093067}{52920} \zeta_{3}+\frac{2250}{49} \zeta_{5}\right] \\
& +n_{f}^{2} \frac{d^{a b c} d_{a b c}}{N_{R}}\left[\frac{133768649869}{120022560000}\right],
\end{aligned}
$$

$\gamma_{\mathrm{ns}}^{(3) \mathrm{s}}(N=9)=$

$$
\begin{aligned}
& n_{f} C_{F} \frac{d^{a b c} d_{a b c}}{N_{R}}\left[-\frac{1021276699865745697}{9924365430000000}+\frac{85388204}{893025} \zeta_{3}\right] \\
& +n_{f} C_{A} \frac{d^{a b c} d_{a b c}}{N_{R}}\left[\frac{2755119043881017}{315059220000000}-\frac{1155263626}{22325625} \zeta_{3}+\frac{19712}{405} \zeta_{5}\right] \\
& +n_{f}^{2} \frac{d^{a b c} d_{a b c}}{N_{R}}\left[\frac{767593737326}{738420046875}\right],
\end{aligned}
$$$$
\gamma_{\mathrm{ns}}^{(3) \mathrm{s}}(N=11)=
$$$$
n_{f} C_{F} \frac{d^{a b c} d_{a b c}}{N_{R}}\left[-\frac{111603773330166434882669}{1394905288902048000000}+\frac{39731371264}{540280125} \zeta_{3}\right]
$$$$
+n_{f} C_{A} \frac{d^{a b c} d_{a b c}}{N_{R}}\left[\frac{4824168874878027761}{1646877554784000000}-\frac{103259298727}{2161120500} \zeta_{3}+\frac{18200}{363} \zeta_{5}\right]
$$

$$
\begin{aligned}
& +n_{f}^{2} \frac{d^{a b c} d_{a b c}}{N_{R}}\left[\frac{40350238520199037}{45289132756560000}\right], \\
& \gamma_{\mathrm{ns}}^{(3) \mathrm{s}}(N=13)= \\
& n_{f} C_{F} \frac{d^{a b c} d_{a b c}}{N_{R}}\left[-\frac{18532792410668780136669601961}{287594205622267242699000000}+\frac{87687308676848}{1491353238375} \zeta_{3}\right]
\end{aligned}
$$




$$
\begin{aligned}
& +n_{f} C_{A} \frac{d^{a b c} d_{a b c}}{N_{R}}\left[-\frac{3391753143539038458111467}{4692679345817880417000000}-\frac{202143595520348}{4474059715125} \zeta_{3}+\frac{422400}{8281} \zeta_{5}\right] \\
& +n_{f}^{2} \frac{d^{a b c} d_{a b c}}{N_{R}}\left[\frac{8444176392147052173457}{11173046061471143850000}\right], \\
& \gamma_{\mathrm{ns}}^{(3) \mathrm{s}}(N=15)= \\
& n_{f} C_{F} \frac{d^{a b c} d_{a b c}}{N_{R}}\left[-\frac{12364402852607089809412352833493}{232027519105674032412672000000}+\frac{89928183421837}{1859349492000} \zeta_{3}\right] \\
& +n_{f} C_{A} \frac{d^{a b c} d_{a b c}}{N_{R}}\left[-\frac{6470906684625313369880498347}{2028212579595052730880000000}-\frac{17737091969947579}{409056888240000} \zeta_{3}+\frac{1547}{30} \zeta_{5}\right] \\
& +n_{f}^{2} \frac{d^{a b c} d_{a b c}}{N_{R}}\left[\frac{1437544924718401819943003}{2228805032522035968000000}\right] .
\end{aligned}
$$

\section{Time-like splitting function}

Here we present the difference between the space- and time-like non-singlet splitting functions at four loops, defined by $\delta P^{(3) \pm}(x) \equiv P_{\sigma=1}^{(3) \pm}(x)-P_{\sigma=-1}^{(3) \pm}(x)$. The expression for $\delta P^{(3)+}(x)$ reads

$$
\begin{aligned}
\delta & P^{(3)+}(x)= \\
& 16 C_{F}^{4}\left\{( 1 + x ) \left(-792 \mathrm{H}_{-2} \zeta_{3}-364 \mathrm{H}_{-2} \zeta_{2}-64 \mathrm{H}_{3} \zeta_{3}+12 \mathrm{H}_{-4,0}+768 \mathrm{H}_{-2,-1} \zeta_{2}-28 \mathrm{H}_{-2,0}\right.\right. \\
& +304 \mathrm{H}_{-2,2}+768 \mathrm{H}_{-1,-2} \zeta_{2}-792 \mathrm{H}_{-1,0} \zeta_{3}-364 \mathrm{H}_{-1,0} \zeta_{2}+608 \mathrm{H}_{-1,3}+1584 \mathrm{H}_{-1,4}-32 \mathrm{H}_{2,0} \zeta_{3} \\
& -324 \mathrm{H}_{2,2}+120 \mathrm{H}_{2,4}-648 \mathrm{H}_{3,1}+112 \mathrm{H}_{3,3}+40 \mathrm{H}_{4,2}-32 \mathrm{H}_{5,1}+192 \mathrm{H}_{-4,-1,0}+64 \mathrm{H}_{-3,-2,0} \\
& -192 \mathrm{H}_{-3,-1,0}-192 \mathrm{H}_{-2,-2,0}-120 \mathrm{H}_{-2,-1,0}-672 \mathrm{H}_{-2,-1,2}+384 \mathrm{H}_{-2,2,1}-192 \mathrm{H}_{-1,-3,0} \\
& -120 \mathrm{H}_{-1,-2,0}-672 \mathrm{H}_{-1,-2,2}+768 \mathrm{H}_{-1,-1,0} \zeta_{2}-1344 \mathrm{H}_{-1,-1,3}-56 \mathrm{H}_{-1,0,0}-1280 \mathrm{H}_{-1,0,0} \zeta_{2} \\
& +304 \mathrm{H}_{-1,2,0}+384 \mathrm{H}_{-1,2,2}+912 \mathrm{H}_{-1,3,0}+768 \mathrm{H}_{-1,3,1}-64 \mathrm{H}_{2,0,0} \zeta_{2}-324 \mathrm{H}_{2,1,0}+56 \mathrm{H}_{2,3,0} \\
& +48 \mathrm{H}_{3,2,0}+76 \mathrm{H}_{4,0,0}+40 \mathrm{H}_{4,1,0}+192 \mathrm{H}_{-2,-1,-1,0}-624 \mathrm{H}_{-2,-1,0,0}+192 \mathrm{H}_{-1,-2,-1,0} \\
& -624 \mathrm{H}_{-1,-2,0,0}+192 \mathrm{H}_{-1,-1,-2,0}-240 \mathrm{H}_{-1,-1,0,0}-672 \mathrm{H}_{-1,-1,2,0}+636 \mathrm{H}_{-1,0,0,0} \\
& +384 \mathrm{H}_{-1,2,0,0}+384 \mathrm{H}_{-1,2,1,0}+24 \mathrm{H}_{2,2,0,0}+116 \mathrm{H}_{3,0,0,0}+48 \mathrm{H}_{3,1,0,0}+384 \mathrm{H}_{-1,-1,-1,0,0} \\
& \left.-1296 \mathrm{H}_{-1,-1,0,0,0}+704 \mathrm{H}_{-1,0,0,0,0}+112 \mathrm{H}_{2,0,0,0,0}+24 \mathrm{H}_{2,1,0,0,0}\right)+(1-x)\left(288 \mathrm{H}_{-3} \zeta_{3}+\frac{9}{2} \mathrm{H}_{0}\right. \\
& -393 \mathrm{H}_{2}-56 \mathrm{H}_{2} \zeta_{3}-76 \mathrm{H}_{2} \zeta_{2}+96 \mathrm{H}_{4} \zeta_{2}-384 \mathrm{H}_{-3,-1} \zeta_{2}-192 \mathrm{H}_{-2,-2} \zeta_{2}+144 \mathrm{H}_{-2,0} \zeta_{3} \\
& -288 \mathrm{H}_{-2,4}-393 \mathrm{H}_{1,0}-56 \mathrm{H}_{1,0} \zeta_{3}-76 \mathrm{H}_{1,0} \zeta_{2}-560 \mathrm{H}_{1,2}-96 \mathrm{H}_{1,2} \zeta_{2}-96 \mathrm{H}_{1,4}-560 \mathrm{H}_{2,1} \\
& -96 \mathrm{H}_{2,1} \zeta_{2}-64 \mathrm{H}_{2,3}+384 \mathrm{H}_{-3,-1,2}+192 \mathrm{H}_{-2,-2,2}-192 \mathrm{H}_{-2,-1,0} \zeta_{2}+384 \mathrm{H}_{-2,-1,3} \\
& +256 \mathrm{H}_{-2,0,0} \zeta_{2}-96 \mathrm{H}_{-2,3,0}-560 \mathrm{H}_{1,0,0}-64 \mathrm{H}_{1,0,0} \zeta_{2}-560 \mathrm{H}_{1,1,0}-96 \mathrm{H}_{1,1,0} \zeta_{2}-64 \mathrm{H}_{1,3,0} \\
& -48 \mathrm{H}_{2,2,0}+96 \mathrm{H}_{-2,-2,0,0}+192 \mathrm{H}_{-2,-1,2,0}+168 \mathrm{H}_{1,0,0,0}+288 \mathrm{H}_{-2,-1,0,0,0}-64 \mathrm{H}_{-2,0,0,0,0} \\
& \left.-32 \mathrm{H}_{1,0,0,0,0}+96 \mathrm{H}_{1,1,0,0,0}\right)-(20+264 x) \mathrm{H}_{0} \zeta_{5}-(28-268 x) \mathrm{H}_{-3,0}-(32+96 x) \mathrm{H}_{3,0} \zeta_{2}
\end{aligned}
$$




$$
\begin{aligned}
& -(32+224 x) \mathrm{H}_{-5,0}+\left(\frac{69}{2}+\frac{1641}{2} x\right) \mathrm{H}_{0,0}-\left(\frac{77}{2}-\frac{725}{2} x\right) \mathrm{H}_{0,0,0}-(46+306 x) \mathrm{H}_{0,0} \zeta_{4} \\
& +(48-1240 x) \mathrm{H}_{0,0,0,0}+(61+839 x) \mathrm{H}_{0} \zeta_{3}+(70+310 x) \mathrm{H}_{0,0,0,0,0,0}+(72+792 x) \mathrm{H}_{0,0} \zeta_{3} \\
& +(80+48 x) \mathrm{H}_{5,0}-(88-72 x) \mathrm{H}_{2,0} \zeta_{2}-(98+82 x) \mathrm{H}_{2,0,0,0}-(110+130 x) \mathrm{H}_{0,0,0,0,0} \\
& -(112-80 x)\left(\mathrm{H}_{3} \zeta_{2}+\mathrm{H}_{-3,2,0}\right)-(120+168 x) \mathrm{H}_{0,0,0} \zeta_{3}+(126+906 x) \mathrm{H}_{0,0,0} \zeta_{2} \\
& -(144+112 x) \mathrm{H}_{0,0,0,0} \zeta_{2}+(168-56 x) \mathrm{H}_{0} \zeta_{2} \zeta_{3}-(176+208 x)\left(\mathrm{H}_{3,2}+\mathrm{H}_{3,1,0}\right) \\
& +(184+480 x) \mathrm{H}_{-2,0,0}+(188-376 x) \mathrm{H}_{0} \zeta_{2}-\left(\frac{403}{2}+\frac{851}{2} x\right) \mathrm{H}_{0} \zeta_{4}+(240+80 x) \mathrm{H}_{6} \\
& -(244+276 x) \mathrm{H}_{3,0,0}+(248+1284 x) \mathrm{H}_{0,0} \zeta_{2}-(260+388 x) \mathrm{H}_{2,0,0}-(264+904 x) \mathrm{H}_{5} \\
& -(280+88 x) \mathrm{H}_{-4,0,0}+(280+600 x) \mathrm{H}_{-3,0,0}-(296-56 x) \mathrm{H}_{-3,0,0,0}+(320-64 x) \mathrm{H}_{-3,-1,0,0} \\
& -(336-240 x) \mathrm{H}_{-4,2}-(342+550 x) \mathrm{H}_{4,0}+(352+992 x) \mathrm{H}_{-3,2}+(368+688 x) \mathrm{H}_{-2,2,0} \\
& -(378+1506 x) \mathrm{H}_{4}-(400-720 x) \mathrm{H}_{3}+(400-304 x) \mathrm{H}_{-3,0} \zeta_{2}-(416-352 x) \mathrm{H}_{-3,3} \\
& +(432-144 x) \mathrm{H}_{-4} \zeta_{2}-(432+720 x) \mathrm{H}_{4,1}-(442+834 x) \mathrm{H}_{3,0}-(448+1088 x) \mathrm{H}_{-3} \zeta_{2} \\
& -(480-640 x) \mathrm{H}_{2,0}+(584+1064 x) \mathrm{H}_{-2,0,0,0}-(864+1184 x) \mathrm{H}_{-2,0} \zeta_{2}+(880+1520 x) \mathrm{H}_{-2,3} \\
& +p_{\mathrm{qq}}(-x)\left(-456 \mathrm{H}_{-4} \zeta_{2}-1248 \mathrm{H}_{-3} \zeta_{3}+192 \mathrm{H}_{-3} \zeta_{2}+910 \mathrm{H}_{-2} \zeta_{4}+96 \mathrm{H}_{-2} \zeta_{3}+36 \mathrm{H}_{-2} \zeta_{2}\right. \\
& -244 \mathrm{H}_{0} \zeta_{5}+\frac{45}{2} \mathrm{H}_{0} \zeta_{4}-27 \mathrm{H}_{0} \zeta_{3}-\frac{39}{2} \mathrm{H}_{0} \zeta_{2}-308 \mathrm{H}_{0} \zeta_{2} \zeta_{3}+24 \mathrm{H}_{3}-48 \mathrm{H}_{3} \zeta_{3}+72 \mathrm{H}_{3} \zeta_{2}+54 \mathrm{H}_{4} \\
& -120 \mathrm{H}_{4} \zeta_{2}+48 \mathrm{H}_{5}-160 \mathrm{H}_{6}-192 \mathrm{H}_{-5,0}+36 \mathrm{H}_{-4,0}+528 \mathrm{H}_{-4,2}+1152 \mathrm{H}_{-3,-1} \zeta_{2} \\
& -1240 \mathrm{H}_{-3,0} \zeta_{2}-192 \mathrm{H}_{-3,2}+1312 \mathrm{H}_{-3,3}+1152 \mathrm{H}_{-2,-2} \zeta_{2}+1248 \mathrm{H}_{-2,-1} \zeta_{3}-192 \mathrm{H}_{-2,-1} \zeta_{2} \\
& -15 \mathrm{H}_{-2,0}-960 \mathrm{H}_{-2,0} \zeta_{3}+216 \mathrm{H}_{-2,0} \zeta_{2}-36 \mathrm{H}_{-2,2}-16 \mathrm{H}_{-2,2} \zeta_{2}-240 \mathrm{H}_{-2,3}+1712 \mathrm{H}_{-2,4} \\
& +1152 \mathrm{H}_{-1,-3} \zeta_{2}+1248 \mathrm{H}_{-1,-2} \zeta_{3}-192 \mathrm{H}_{-1,-2} \zeta_{2}+910 \mathrm{H}_{-1,0} \zeta_{4}+96 \mathrm{H}_{-1,0} \zeta_{3}+36 \mathrm{H}_{-1,0} \zeta_{2} \\
& -72 \mathrm{H}_{-1,3}-32 \mathrm{H}_{-1,3} \zeta_{2}-144 \mathrm{H}_{-1,4}+1088 \mathrm{H}_{-1,5}-18 \mathrm{H}_{0,0}-612 \mathrm{H}_{0,0} \zeta_{4}-36 \mathrm{H}_{0,0} \zeta_{2}+12 \mathrm{H}_{2,0} \\
& -24 \mathrm{H}_{2,0} \zeta_{3}+36 \mathrm{H}_{2,0} \zeta_{2}-96 \mathrm{H}_{2,2} \zeta_{2}+18 \mathrm{H}_{3,0}-136 \mathrm{H}_{3,0} \zeta_{2}-192 \mathrm{H}_{3,1} \zeta_{2}-48 \mathrm{H}_{3,2}-60 \mathrm{H}_{4,0} \\
& -144 \mathrm{H}_{4,1}-96 \mathrm{H}_{4,2}-224 \mathrm{H}_{5,0}-384 \mathrm{H}_{5,1}+144 \mathrm{H}_{-4,-1,0}+96 \mathrm{H}_{-4,0,0}+48 \mathrm{H}_{-3,-2,0} \\
& -1152 \mathrm{H}_{-3,-1,2}-72 \mathrm{H}_{-3,0,0}+560 \mathrm{H}_{-3,2,0}+768 \mathrm{H}_{-3,2,1}+48 \mathrm{H}_{-2,-3,0}-1152 \mathrm{H}_{-2,-2,2} \\
& -1152 \mathrm{H}_{-2,-1,-1} \zeta_{2}+1664 \mathrm{H}_{-2,-1,0} \zeta_{2}+192 \mathrm{H}_{-2,-1,2}-2112 \mathrm{H}_{-2,-1,3}-18 \mathrm{H}_{-2,0,0} \\
& -1408 \mathrm{H}_{-2,0,0} \zeta_{2}-48 \mathrm{H}_{-2,2,0}+96 \mathrm{H}_{-2,2,1}+384 \mathrm{H}_{-2,2,2}+1056 \mathrm{H}_{-2,3,0}+1152 \mathrm{H}_{-2,3,1} \\
& +144 \mathrm{H}_{-1,-4,0}-1152 \mathrm{H}_{-1,-3,2}-1152 \mathrm{H}_{-1,-2,-1} \zeta_{2}+1664 \mathrm{H}_{-1,-2,0} \zeta_{2}+192 \mathrm{H}_{-1,-2,2} \\
& -2112 \mathrm{H}_{-1,-2,3}-1152 \mathrm{H}_{-1,-1,-2} \zeta_{2}+1248 \mathrm{H}_{-1,-1,0} \zeta_{3}-192 \mathrm{H}_{-1,-1,0} \zeta_{2}+384 \mathrm{H}_{-1,-1,3} \\
& -2880 \mathrm{H}_{-1,-1,4}-30 \mathrm{H}_{-1,0,0}-672 \mathrm{H}_{-1,0,0} \zeta_{3}+240 \mathrm{H}_{-1,0,0} \zeta_{2}-36 \mathrm{H}_{-1,2,0}-16 \mathrm{H}_{-1,2,0} \zeta_{2} \\
& +96 \mathrm{H}_{-1,2,2}+48 \mathrm{H}_{-1,3,0}+192 \mathrm{H}_{-1,3,1}+384 \mathrm{H}_{-1,3,2}+848 \mathrm{H}_{-1,4,0}+1152 \mathrm{H}_{-1,4,1}+\frac{81}{2} \mathrm{H}_{0,0,0} \\
& -72 \mathrm{H}_{0,0,0} \zeta_{2}-96 \mathrm{H}_{2,0,0} \zeta_{2}-96 \mathrm{H}_{2,1,0} \zeta_{2}-48 \mathrm{H}_{3,0,0}-48 \mathrm{H}_{3,1,0}-48 \mathrm{H}_{4,0,0}-96 \mathrm{H}_{4,1,0} \\
& -480 \mathrm{H}_{-3,-1,0,0}+448 \mathrm{H}_{-3,0,0,0}-576 \mathrm{H}_{-2,-2,0,0}+1152 \mathrm{H}_{-2,-1,-1,2}+96 \mathrm{H}_{-2,-1,0,0} \\
& -960 \mathrm{H}_{-2,-1,2,0}-768 \mathrm{H}_{-2,-1,2,1}-144 \mathrm{H}_{-2,0,0,0}+352 \mathrm{H}_{-2,2,0,0}+384 \mathrm{H}_{-2,2,1,0}-480 \mathrm{H}_{-1,-3,0,0} \\
& +1152 \mathrm{H}_{-1,-2,-1,2}+96 \mathrm{H}_{-1,-2,0,0}-960 \mathrm{H}_{-1,-2,2,0}-768 \mathrm{H}_{-1,-2,2,1}+1152 \mathrm{H}_{-1,-1,-2,2} \\
& -1152 \mathrm{H}_{-1,-1,-1,0} \zeta_{2}+2304 \mathrm{H}_{-1,-1,-1,3}+2176 \mathrm{H}_{-1,-1,0,0} \zeta_{2}+192 \mathrm{H}_{-1,-1,2,0}-768 \mathrm{H}_{-1,-1,2,2}
\end{aligned}
$$




$$
\begin{aligned}
& -1728 \mathrm{H}_{-1,-1,3,0}-1536 \mathrm{H}_{-1,-1,3,1}-54 \mathrm{H}_{-1,0,0,0}-960 \mathrm{H}_{-1,0,0,0} \zeta_{2}+96 \mathrm{H}_{-1,2,0,0}+96 \mathrm{H}_{-1,2,1,0} \\
& +320 \mathrm{H}_{-1,3,0,0}+384 \mathrm{H}_{-1,3,1,0}+64 \mathrm{H}_{0,0,0,0} \zeta_{2}+48 \mathrm{H}_{3,0,0,0}+576 \mathrm{H}_{-2,-1,-1,0,0} \\
& -1120 \mathrm{H}_{-2,-1,0,0,0}+496 \mathrm{H}_{-2,0,0,0,0}+576 \mathrm{H}_{-1,-2,-1,0,0}-1120 \mathrm{H}_{-1,-2,0,0,0}+576 \mathrm{H}_{-1,-1,-2,0,0} \\
& +1152 \mathrm{H}_{-1,-1,-1,2,0}+288 \mathrm{H}_{-1,-1,0,0,0}-768 \mathrm{H}_{-1,-1,2,0,0}-768 \mathrm{H}_{-1,-1,2,1,0}-96 \mathrm{H}_{-1,2,0,0,0} \\
& \left.-60 \mathrm{H}_{0,0,0,0,0}+1728 \mathrm{H}_{-1,-1,-1,0,0,0}-1024 \mathrm{H}_{-1,-1,0,0,0,0}-80 \mathrm{H}_{-1,0,0,0,0,0}+192 \mathrm{H}_{0,0,0,0,0,0}\right) \\
& +p_{\mathrm{qq}}(x)\left(-264 \mathrm{H}_{-4} \zeta_{2}-336 \mathrm{H}_{-3} \zeta_{3}+72 \mathrm{H}_{-3} \zeta_{2}+\frac{29}{8} \mathrm{H}_{0}+144 \mathrm{H}_{0} \zeta_{5}-\frac{45}{2} \mathrm{H}_{0} \zeta_{4}+25 \mathrm{H}_{0} \zeta_{3}\right. \\
& +\frac{9}{2} \mathrm{H}_{0} \zeta_{2}-124 \mathrm{H}_{0} \zeta_{2} \zeta_{3}-66 \mathrm{H}_{2} \zeta_{4}+24 \mathrm{H}_{2} \zeta_{3}-64 \mathrm{H}_{3} \zeta_{3}+15 \mathrm{H}_{4}+24 \mathrm{H}_{4} \zeta_{2}+96 \mathrm{H}_{5}-320 \mathrm{H}_{6} \\
& +192 \mathrm{H}_{-5,0}-36 \mathrm{H}_{-4,0}+48 \mathrm{H}_{-4,2}+384 \mathrm{H}_{-3,-1} \zeta_{2}-280 \mathrm{H}_{-3,0} \zeta_{2}+128 \mathrm{H}_{-3,3}+192 \mathrm{H}_{-2,-2} \zeta_{2} \\
& -168 \mathrm{H}_{-2,0} \zeta_{3}+36 \mathrm{H}_{-2,0} \zeta_{2}+144 \mathrm{H}_{-2,4}+76 \mathrm{H}_{0,0} \zeta_{4}-28 \mathrm{H}_{0,0} \zeta_{2}+32 \mathrm{H}_{1,-3} \zeta_{2}-66 \mathrm{H}_{1,0} \zeta_{4} \\
& +24 \mathrm{H}_{1,0} \zeta_{3}-64 \mathrm{H}_{1,2} \zeta_{3}+144 \mathrm{H}_{1,4}-256 \mathrm{H}_{1,5}+16 \mathrm{H}_{2,-2} \zeta_{2}-32 \mathrm{H}_{2,0} \zeta_{3}-64 \mathrm{H}_{2,1} \zeta_{3}+96 \mathrm{H}_{2,3} \\
& -256 \mathrm{H}_{2,4}-13 \mathrm{H}_{3,0}+72 \mathrm{H}_{3,0} \zeta_{2}+24 \mathrm{H}_{3,2}-224 \mathrm{H}_{3,3}+60 \mathrm{H}_{4,0}-72 \mathrm{H}_{4,1}-224 \mathrm{H}_{4,2}-288 \mathrm{H}_{5,0} \\
& -320 \mathrm{H}_{5,1}-432 \mathrm{H}_{-4,-1,0}+336 \mathrm{H}_{-4,0,0}-336 \mathrm{H}_{-3,-2,0}+144 \mathrm{H}_{-3,-1,0}-192 \mathrm{H}_{-3,-1,2} \\
& -96 \mathrm{H}_{-3,0,0}+16 \mathrm{H}_{-3,2,0}-192 \mathrm{H}_{-2,-3,0}+72 \mathrm{H}_{-2,-2,0}-96 \mathrm{H}_{-2,-2,2}+192 \mathrm{H}_{-2,-1,0} \zeta_{2} \\
& -192 \mathrm{H}_{-2,-1,3}-192 \mathrm{H}_{-2,0,0} \zeta_{2}+48 \mathrm{H}_{-2,3,0}+\frac{45}{4} \mathrm{H}_{0,0,0}+160 \mathrm{H}_{0,0,0} \zeta_{3}+36 \mathrm{H}_{0,0,0} \zeta_{2} \\
& +144 \mathrm{H}_{1,-4,0}+96 \mathrm{H}_{1,-3,0}-128 \mathrm{H}_{1,-3,2}+16 \mathrm{H}_{1,-2,0} \zeta_{2}-128 \mathrm{H}_{1,-2,3}-64 \mathrm{H}_{1,1,0} \zeta_{3}-192 \mathrm{H}_{1,1,4} \\
& +32 \mathrm{H}_{1,2,0} \zeta_{2}-128 \mathrm{H}_{1,2,3}+96 \mathrm{H}_{1,3,0}-160 \mathrm{H}_{1,3,2}-304 \mathrm{H}_{1,4,0}-288 \mathrm{H}_{1,4,1}+48 \mathrm{H}_{2,-3,0} \\
& +48 \mathrm{H}_{2,-2,0}-64 \mathrm{H}_{2,-2,2}-49 \mathrm{H}_{2,0,0}+112 \mathrm{H}_{2,0,0} \zeta_{2}+32 \mathrm{H}_{2,1,0} \zeta_{2}-128 \mathrm{H}_{2,1,3}+72 \mathrm{H}_{2,2,0} \\
& -96 \mathrm{H}_{2,2,2}-240 \mathrm{H}_{2,3,0}-160 \mathrm{H}_{2,3,1}+120 \mathrm{H}_{3,0,0}+24 \mathrm{H}_{3,1,0}-96 \mathrm{H}_{3,1,2}-192 \mathrm{H}_{3,2,0}-96 \mathrm{H}_{3,2,1} \\
& -240 \mathrm{H}_{4,0,0}-224 \mathrm{H}_{4,1,0}-96 \mathrm{H}_{4,1,1}+384 \mathrm{H}_{-3,-1,-1,0}-576 \mathrm{H}_{-3,-1,0,0}+336 \mathrm{H}_{-3,0,0,0} \\
& +192 \mathrm{H}_{-2,-2,-1,0}-336 \mathrm{H}_{-2,-2,0,0}+192 \mathrm{H}_{-2,-1,-2,0}+144 \mathrm{H}_{-2,-1,0,0}-96 \mathrm{H}_{-2,-1,2,0} \\
& -108 \mathrm{H}_{-2,0,0,0}-70 \mathrm{H}_{0,0,0,0}+256 \mathrm{H}_{0,0,0,0} \zeta_{2}-192 \mathrm{H}_{1,-3,-1,0}+128 \mathrm{H}_{1,-3,0,0}-96 \mathrm{H}_{1,-2,-2,0} \\
& +96 \mathrm{H}_{1,-2,0,0}-64 \mathrm{H}_{1,-2,2,0}-93 \mathrm{H}_{1,0,0,0}+144 \mathrm{H}_{1,0,0,0} \zeta_{2}+64 \mathrm{H}_{1,1,0,0} \zeta_{2}-128 \mathrm{H}_{1,1,3,0} \\
& +96 \mathrm{H}_{1,2,0,0}-96 \mathrm{H}_{1,2,2,0}-160 \mathrm{H}_{1,3,0,0}-160 \mathrm{H}_{1,3,1,0}-96 \mathrm{H}_{2,-2,-1,0}+16 \mathrm{H}_{2,-2,0,0}+180 \mathrm{H}_{2,0,0,0} \\
& +96 \mathrm{H}_{2,1,0,0}-96 \mathrm{H}_{2,1,2,0}-96 \mathrm{H}_{2,2,0,0}-96 \mathrm{H}_{2,2,1,0}-176 \mathrm{H}_{3,0,0,0}-96 \mathrm{H}_{3,1,0,0}-96 \mathrm{H}_{3,1,1,0} \\
& +384 \mathrm{H}_{-2,-1,-1,0,0}-432 \mathrm{H}_{-2,-1,0,0,0}+192 \mathrm{H}_{-2,0,0,0,0}+180 \mathrm{H}_{0,0,0,0,0}-192 \mathrm{H}_{1,-2,-1,0,0} \\
& +48 \mathrm{H}_{1,-2,0,0,0}+144 \mathrm{H}_{1,0,0,0,0}+144 \mathrm{H}_{1,1,0,0,0}-16 \mathrm{H}_{1,2,0,0,0}-144 \mathrm{H}_{2,0,0,0,0}-16 \mathrm{H}_{2,1,0,0,0} \\
& \left.\left.-272 \mathrm{H}_{0,0,0,0,0,0}-240 \mathrm{H}_{1,0,0,0,0,0}-64 \mathrm{H}_{1,1,0,0,0,0}+192 \mathrm{H}_{1,1,1,0,0,0}\right)\right\} \\
& +16 C_{F}^{3} C_{A}\left\{( 1 + x ) \left(868 \mathrm{H}_{-2} \zeta_{3}+\frac{874}{3} \mathrm{H}_{-2} \zeta_{2}-32 \mathrm{H}_{3} \zeta_{3}-864 \mathrm{H}_{-2,-1} \zeta_{2}+238 \mathrm{H}_{-2,0}-288 \mathrm{H}_{-2,2}\right.\right. \\
& -864 \mathrm{H}_{-1,-2} \zeta_{2}+868 \mathrm{H}_{-1,0} \zeta_{3}+\frac{874}{3} \mathrm{H}_{-1,0} \zeta_{2}-576 \mathrm{H}_{-1,3}-1704 \mathrm{H}_{-1,4}-16 \mathrm{H}_{2,0} \zeta_{3}+\frac{1016}{3} \mathrm{H}_{2,2} \\
& -16 \mathrm{H}_{2,2} \zeta_{2}-144 \mathrm{H}_{2,4}+\frac{2032}{3} \mathrm{H}_{3,1}-32 \mathrm{H}_{3,1} \zeta_{2}-96 \mathrm{H}_{3,3}+48 \mathrm{H}_{4,2}+192 \mathrm{H}_{5,1}+\frac{20}{3} \mathrm{H}_{-2,-1,0} \\
& +752 \mathrm{H}_{-2,-1,2}-384 \mathrm{H}_{-2,2,1}+224 \mathrm{H}_{-1,-3,0}+\frac{20}{3} \mathrm{H}_{-1,-2,0}+752 \mathrm{H}_{-1,-2,2}-864 \mathrm{H}_{-1,-1,0} \zeta_{2}
\end{aligned}
$$




$$
\begin{aligned}
& +1504 \mathrm{H}_{-1,-1,3}+476 \mathrm{H}_{-1,0,0}+1376 \mathrm{H}_{-1,0,0} \zeta_{2}-288 \mathrm{H}_{-1,2,0}-384 \mathrm{H}_{-1,2,2}-952 \mathrm{H}_{-1,3,0} \\
& -768 \mathrm{H}_{-1,3,1}-64 \mathrm{H}_{2,-3,0}+48 \mathrm{H}_{2,0,0} \zeta_{2}+\frac{1016}{3} \mathrm{H}_{2,1,0}-16 \mathrm{H}_{2,1,0} \zeta_{2}-48 \mathrm{H}_{2,3,0}-64 \mathrm{H}_{3,-2,0} \\
& +72 \mathrm{H}_{4,0,0}+48 \mathrm{H}_{4,1,0}-224 \mathrm{H}_{-2,-1,-1,0}-224 \mathrm{H}_{-1,-2,-1,0}+712 \mathrm{H}_{-1,-2,0,0}-224 \mathrm{H}_{-1,-1,-2,0} \\
& +\frac{40}{3} \mathrm{H}_{-1,-1,0,0}+752 \mathrm{H}_{-1,-1,2,0}-266 \mathrm{H}_{-1,0,0,0}-384 \mathrm{H}_{-1,2,0,0}-384 \mathrm{H}_{-1,2,1,0}-64 \mathrm{H}_{2,-2,0,0} \\
& +16 \mathrm{H}_{2,2,0,0}+24 \mathrm{H}_{3,0,0,0}+32 \mathrm{H}_{3,1,0,0}-448 \mathrm{H}_{-1,-1,-1,0,0}+1464 \mathrm{H}_{-1,-1,0,0,0}-864 \mathrm{H}_{-1,0,0,0,0} \\
& \left.+48 \mathrm{H}_{2,1,0,0,0}\right)+(1-x)\left(-392 \mathrm{H}_{-3} \zeta_{3}+\frac{373}{12} \mathrm{H}_{0}+\frac{1373}{3} \mathrm{H}_{2}+196 \mathrm{H}_{2} \zeta_{3}+78 \mathrm{H}_{2} \zeta_{2}\right. \\
& +512 \mathrm{H}_{-3,-1} \zeta_{2}+256 \mathrm{H}_{-2,-2} \zeta_{2}-196 \mathrm{H}_{-2,0} \zeta_{3}+360 \mathrm{H}_{-2,4}+\frac{1373}{3} \mathrm{H}_{1,0}+196 \mathrm{H}_{1,0} \zeta_{3} \\
& +78 \mathrm{H}_{1,0} \zeta_{2}+\frac{1672}{3} \mathrm{H}_{1,2}+112 \mathrm{H}_{1,2} \zeta_{2}+96 \mathrm{H}_{1,4}+\frac{1672}{3} \mathrm{H}_{2,1}+112 \mathrm{H}_{2,1} \zeta_{2}+32 \mathrm{H}_{2,3} \\
& -480 \mathrm{H}_{-3,-1,2}-32 \mathrm{H}_{-2,-3,0}-240 \mathrm{H}_{-2,-2,2}+256 \mathrm{H}_{-2,-1,0} \zeta_{2}-480 \mathrm{H}_{-2,-1,3}-320 \mathrm{H}_{-2,0,0} \zeta_{2} \\
& +120 \mathrm{H}_{-2,3,0}+128 \mathrm{H}_{1,-3,0}+572 \mathrm{H}_{1,0,0}+112 \mathrm{H}_{1,0,0} \zeta_{2}+\frac{1672}{3} \mathrm{H}_{1,1,0}+112 \mathrm{H}_{1,1,0} \zeta_{2}+32 \mathrm{H}_{1,3,0} \\
& +64 \mathrm{H}_{2,-2,0}+64 \mathrm{H}_{-3,-1,-1,0}+32 \mathrm{H}_{-2,-2,-1,0}-168 \mathrm{H}_{-2,-2,0,0}+32 \mathrm{H}_{-2,-1,-2,0} \\
& -240 \mathrm{H}_{-2,-1,2,0}+96 \mathrm{H}_{0,0,0,0} \zeta_{2}+128 \mathrm{H}_{1,-2,0,0}-204 \mathrm{H}_{1,0,0,0}-96 \mathrm{H}_{1,2,0,0}-96 \mathrm{H}_{2,1,0,0} \\
& \left.+64 \mathrm{H}_{-2,-1,-1,0,0}-408 \mathrm{H}_{-2,-1,0,0,0}+160 \mathrm{H}_{-2,0,0,0,0}-256 \mathrm{H}_{1,0,0,0,0}-288 \mathrm{H}_{1,1,0,0,0}\right) \\
& +\left(6+\frac{1550}{3} x\right) \mathrm{H}_{0,0,0,0}+\left(\frac{22}{3}+\frac{290}{3} x\right) \mathrm{H}_{-3,0}+(8+72 x) \mathrm{H}_{3,0} \zeta_{2}+\left(\frac{31}{3}-927 x\right) \mathrm{H}_{0,0} \\
& +(32+96 x) \mathrm{H}_{-5,0}+(50+88 x) \mathrm{H}_{0} \zeta_{5}+(54-90 x) \mathrm{H}_{0,0,0} \zeta_{3}-(62-354 x) \mathrm{H}_{2,0,0,0} \\
& -\left(\frac{196}{3}+\frac{3004}{3} x\right) \mathrm{H}_{0,0} \zeta_{3}+(72+128 x) \mathrm{H}_{5,0}-\left(\frac{463}{6}+\frac{4201}{6} x\right) \mathrm{H}_{0} \zeta_{3}-\left(\frac{244}{3}-8 x\right) \mathrm{H}_{-2,0,0} \\
& +\left(\frac{335}{4}-\frac{3877}{4} x\right) \mathrm{H}_{0,0,0}-(100+32 x) \mathrm{H}_{-4,0}+(112-128 x) \mathrm{H}_{-3,2,0}-(112+16 x) \mathrm{H}_{-3,-2,0} \\
& -(120-160 x) \mathrm{H}_{6}-(120-72 x) \mathrm{H}_{4} \zeta_{2}-\left(\frac{249}{2}-\frac{939}{2} x\right) \mathrm{H}_{0} \zeta_{2}+(126-98 x) \mathrm{H}_{2,0} \zeta_{2} \\
& +(126+234 x) \mathrm{H}_{0,0} \zeta_{4}-(132-156 x) \mathrm{H}_{0} \zeta_{2} \zeta_{3}+\left(\frac{400}{3}+\frac{2960}{3} x\right) \mathrm{H}_{5}+(140-84 x) \mathrm{H}_{3} \zeta_{2} \\
& +(144+240 x)\left(\mathrm{H}_{3,2}+\mathrm{H}_{3,1,0}\right)-(144+912 x) \mathrm{H}_{0,0,0} \zeta_{2}+\left(\frac{436}{3}+\frac{1300}{3} x\right) \mathrm{H}_{3,0,0} \\
& +\left(\frac{983}{6}+\frac{728}{3} x\right) \mathrm{H}_{0} \zeta_{4}-(240+144 x) \mathrm{H}_{-4,-1,0}+\left(\frac{748}{3}+\frac{1820}{3} x\right) \mathrm{H}_{4,0} \\
& +(252+196 x) \mathrm{H}_{-2,-2,0}+(278+414 x) \mathrm{H}_{2,0,0}+(280+168 x) \mathrm{H}_{-3,-1,0} \\
& -\left(\frac{860}{3}+1078 x\right) \mathrm{H}_{0,0} \zeta_{2}+(304-72 x) \mathrm{H}_{-4,0,0}+(336-384 x) \mathrm{H}_{-4,2}-\left(\frac{1072}{3}+\frac{3440}{3} x\right) \mathrm{H}_{-3,2} \\
& +(368-200 x) \mathrm{H}_{-3,0,0,0}-\left(\frac{1112}{3}+\frac{2296}{3} x\right) \mathrm{H}_{-2,2,0}-(432-176 x) \mathrm{H}_{-3,-1,0,0} \\
& +(432+720 x) \mathrm{H}_{4,1}-(444+620 x) \mathrm{H}_{-3,0,0}+(452+1472 x) \mathrm{H}_{4}-(456-312 x) \mathrm{H}_{-4} \zeta_{2} \\
& +(464-496 x) \mathrm{H}_{-3,3}+(465-679 x) \mathrm{H}_{3}-(472-424 x) \mathrm{H}_{-3,0} \zeta_{2}+\left(\frac{1490}{3}+\frac{2510}{3} x\right) \mathrm{H}_{3,0}
\end{aligned}
$$




$$
\begin{aligned}
& +\left(\frac{1492}{3}+\frac{3692}{3} x\right) \mathrm{H}_{-3} \zeta_{2}+\left(\frac{1037}{2}-\frac{1251}{2} x\right) \mathrm{H}_{2,0}+(768+656 x) \mathrm{H}_{-2,-1,0,0} \\
& -(782+1114 x) \mathrm{H}_{-2,0,0,0}-\left(\frac{2776}{3}+\frac{5144}{3} x\right) \mathrm{H}_{-2,3}+\left(\frac{2810}{3}+\frac{3910}{3} x\right) \mathrm{H}_{-2,0} \zeta_{2} \\
& -48 x \mathrm{H}_{0,0,0,0,0,0}+140 x \mathrm{H}_{0,0,0,0,0}+p_{\mathrm{qq}}(-x)\left(660 \mathrm{H}_{-4} \zeta_{2}+1456 \mathrm{H}_{-3} \zeta_{3}-288 \mathrm{H}_{-3} \zeta_{2}\right. \\
& -835 \mathrm{H}_{-2} \zeta_{4}-\frac{136}{3} \mathrm{H}_{-2} \zeta_{3}-\frac{1258}{3} \mathrm{H}_{-2} \zeta_{2}+36 \mathrm{H}_{0} \zeta_{5}+\frac{65}{12} \mathrm{H}_{0} \zeta_{4}+\frac{2155}{6} \mathrm{H}_{0} \zeta_{3}-\frac{79}{4} \mathrm{H}_{0} \zeta_{2} \\
& +390 \mathrm{H}_{0} \zeta_{2} \zeta_{3}-36 \mathrm{H}_{3}+72 \mathrm{H}_{3} \zeta_{3}-60 \mathrm{H}_{3} \zeta_{2}-361 \mathrm{H}_{4}+156 \mathrm{H}_{4} \zeta_{2}-168 \mathrm{H}_{5}+320 \mathrm{H}_{6}+96 \mathrm{H}_{-5,0} \\
& +194 \mathrm{H}_{-4,0}-696 \mathrm{H}_{-4,2}-1344 \mathrm{H}_{-3,-1} \zeta_{2}+\frac{940}{3} \mathrm{H}_{-3,0}+1468 \mathrm{H}_{-3,0} \zeta_{2}+\frac{688}{3} \mathrm{H}_{-3,2} \\
& -1584 \mathrm{H}_{-3,3}-1344 \mathrm{H}_{-2,-2} \zeta_{2}-1456 \mathrm{H}_{-2,-1} \zeta_{3}+\frac{512}{3} \mathrm{H}_{-2,-1} \zeta_{2}-\frac{151}{2} \mathrm{H}_{-2,0}+1184 \mathrm{H}_{-2,0} \zeta_{3} \\
& -\frac{956}{3} \mathrm{H}_{-2,0} \zeta_{2}+\frac{722}{3} \mathrm{H}_{-2,2}+8 \mathrm{H}_{-2,2} \zeta_{2}+\frac{872}{3} \mathrm{H}_{-2,3}-2024 \mathrm{H}_{-2,4}-1344 \mathrm{H}_{-1,-3} \zeta_{2} \\
& -1456 \mathrm{H}_{-1,-2} \zeta_{3}+\frac{512}{3} \mathrm{H}_{-1,-2} \zeta_{2}-835 \mathrm{H}_{-1,0} \zeta_{4}-\frac{136}{3} \mathrm{H}_{-1,0} \zeta_{3}-\frac{1258}{3} \mathrm{H}_{-1,0} \zeta_{2}+\frac{1444}{3} \mathrm{H}_{-1,3} \\
& +16 \mathrm{H}_{-1,3} \zeta_{2}+184 \mathrm{H}_{-1,4}-1376 \mathrm{H}_{-1,5}+27 \mathrm{H}_{0,0}+454 \mathrm{H}_{0,0} \zeta_{4}+\frac{316}{3} \mathrm{H}_{0,0} \zeta_{3}+\frac{1138}{3} \mathrm{H}_{0,0} \zeta_{2} \\
& -18 \mathrm{H}_{2,0}+36 \mathrm{H}_{2,0} \zeta_{3}-30 \mathrm{H}_{2,0} \zeta_{2}+112 \mathrm{H}_{2,2} \zeta_{2}-\frac{361}{3} \mathrm{H}_{3,0}+164 \mathrm{H}_{3,0} \zeta_{2}+224 \mathrm{H}_{3,1} \zeta_{2}+\frac{248}{3} \mathrm{H}_{3,2} \\
& +38 \mathrm{H}_{4,0}+248 \mathrm{H}_{4,1}+112 \mathrm{H}_{4,2}+288 \mathrm{H}_{5,0}+448 \mathrm{H}_{5,1}-72 \mathrm{H}_{-4,-1,0}-264 \mathrm{H}_{-4,0,0}-24 \mathrm{H}_{-3,-2,0} \\
& -\frac{352}{3} \mathrm{H}_{-3,-1,0}+1344 \mathrm{H}_{-3,-1,2}+420 \mathrm{H}_{-3,0,0}-680 \mathrm{H}_{-3,2,0}-896 \mathrm{H}_{-3,2,1}-24 \mathrm{H}_{-2,-3,0} \\
& -\frac{352}{3} \mathrm{H}_{-2,-2,0}+1344 \mathrm{H}_{-2,-2,2}+1344 \mathrm{H}_{-2,-1,-1} \zeta_{2}-\frac{1072}{3} \mathrm{H}_{-2,-1,0}-1920 \mathrm{H}_{-2,-1,0} \zeta_{2} \\
& -\frac{512}{3} \mathrm{H}_{-2,-1,2}+2464 \mathrm{H}_{-2,-1,3}+\frac{1705}{3} \mathrm{H}_{-2,0,0}+1648 \mathrm{H}_{-2,0,0} \zeta_{2}+\frac{184}{3} \mathrm{H}_{-2,2,0}-\frac{496}{3} \mathrm{H}_{-2,2,1} \\
& -448 \mathrm{H}_{-2,2,2}-1232 \mathrm{H}_{-2,3,0}-1344 \mathrm{H}_{-2,3,1}-72 \mathrm{H}_{-1,-4,0}-\frac{352}{3} \mathrm{H}_{-1,-3,0}+1344 \mathrm{H}_{-1,-3,2} \\
& +1344 \mathrm{H}_{-1,-2,-1} \zeta_{2}-\frac{1072}{3} \mathrm{H}_{-1,-2,0}-1920 \mathrm{H}_{-1,-2,0} \zeta_{2}-\frac{512}{3} \mathrm{H}_{-1,-2,2}+2464 \mathrm{H}_{-1,-2,3} \\
& +1344 \mathrm{H}_{-1,-1,-2} \zeta_{2}-1456 \mathrm{H}_{-1,-1,0} \zeta_{3}+\frac{512}{3} \mathrm{H}_{-1,-1,0} \zeta_{2}-\frac{1024}{3} \mathrm{H}_{-1,-1,3}+3360 \mathrm{H}_{-1,-1,4} \\
& -151 \mathrm{H}_{-1,0,0}+912 \mathrm{H}_{-1,0,0} \zeta_{3}-\frac{1048}{3} \mathrm{H}_{-1,0,0} \zeta_{2}+\frac{722}{3} \mathrm{H}_{-1,2,0}+8 \mathrm{H}_{-1,2,0} \zeta_{2}-\frac{496}{3} \mathrm{H}_{-1,2,2} \\
& -\frac{136}{3} \mathrm{H}_{-1,3,0}-\frac{992}{3} \mathrm{H}_{-1,3,1}-448 \mathrm{H}_{-1,3,2}-1016 \mathrm{H}_{-1,4,0}-1344 \mathrm{H}_{-1,4,1}+\frac{345}{4} \mathrm{H}_{0,0,0} \\
& -192 \mathrm{H}_{0,0,0} \zeta_{3}+304 \mathrm{H}_{0,0,0} \zeta_{2}+112 \mathrm{H}_{2,0,0} \zeta_{2}+112 \mathrm{H}_{2,1,0} \zeta_{2}+\frac{160}{3} \mathrm{H}_{3,0,0}+\frac{248}{3} \mathrm{H}_{3,1,0}+64 \mathrm{H}_{4,0,0} \\
& +112 \mathrm{H}_{4,1,0}+624 \mathrm{H}_{-3,-1,0,0}-664 \mathrm{H}_{-3,0,0,0}+672 \mathrm{H}_{-2,-2,0,0}-1344 \mathrm{H}_{-2,-1,-1,2} \\
& -320 \mathrm{H}_{-2,-1,0,0}+1120 \mathrm{H}_{-2,-1,2,0}+896 \mathrm{H}_{-2,-1,2,1}+572 \mathrm{H}_{-2,0,0,0}-416 \mathrm{H}_{-2,2,0,0} \\
& -448 \mathrm{H}_{-2,2,1,0}+624 \mathrm{H}_{-1,-3,0,0}-1344 \mathrm{H}_{-1,-2,-1,2}-320 \mathrm{H}_{-1,-2,0,0}+1120 \mathrm{H}_{-1,-2,2,0} \\
& +896 \mathrm{H}_{-1,-2,2,1}-1344 \mathrm{H}_{-1,-1,-2,2}+1344 \mathrm{H}_{-1,-1,-1,0} \zeta_{2}-2688 \mathrm{H}_{-1,-1,-1,3}-\frac{2144}{3} \mathrm{H}_{-1,-1,0,0} \\
& -2496 \mathrm{H}_{-1,-1,0,0} \zeta_{2}-\frac{512}{3} \mathrm{H}_{-1,-1,2,0}+896 \mathrm{H}_{-1,-1,2,2}+2016 \mathrm{H}_{-1,-1,3,0}+1792 \mathrm{H}_{-1,-1,3,1}
\end{aligned}
$$




$$
\begin{aligned}
& +765 \mathrm{H}_{-1,0,0,0}+1200 \mathrm{H}_{-1,0,0,0} \zeta_{2}-\frac{320}{3} \mathrm{H}_{-1,2,0,0}-\frac{496}{3} \mathrm{H}_{-1,2,1,0}-384 \mathrm{H}_{-1,3,0,0}-448 \mathrm{H}_{-1,3,1,0} \\
& -\frac{700}{3} \mathrm{H}_{0,0,0,0}-224 \mathrm{H}_{0,0,0,0} \zeta_{2}-48 \mathrm{H}_{3,0,0,0}-672 \mathrm{H}_{-2,-1,-1,0,0}+1360 \mathrm{H}_{-2,-1,0,0,0} \\
& -744 \mathrm{H}_{-2,0,0,0,0}-672 \mathrm{H}_{-1,-2,-1,0,0}+1360 \mathrm{H}_{-1,-2,0,0,0}-672 \mathrm{H}_{-1,-1,-2,0,0} \\
& -1344 \mathrm{H}_{-1,-1,-1,2,0}-608 \mathrm{H}_{-1,-1,0,0,0}+896 \mathrm{H}_{-1,-1,2,0,0}+896 \mathrm{H}_{-1,-1,2,1,0}+544 \mathrm{H}_{-1,0,0,0,0} \\
& +96 \mathrm{H}_{-1,2,0,0,0}-310 \mathrm{H}_{0,0,0,0,0}-2016 \mathrm{H}_{-1,-1,-1,0,0,0}+1408 \mathrm{H}_{-1,-1,0,0,0,0}-200 \mathrm{H}_{-1,0,0,0,0,0} \\
& \left.-48 \mathrm{H}_{0,0,0,0,0,0}\right)+p_{\mathrm{qq}}(x)\left(84 \mathrm{H}_{-4} \zeta_{2}+280 \mathrm{H}_{-3} \zeta_{3}+36 \mathrm{H}_{-3} \zeta_{2}+\frac{587}{48} \mathrm{H}_{0}-26 \mathrm{H}_{0} \zeta_{5}+\frac{149}{4} \mathrm{H}_{0} \zeta_{4}\right. \\
& -\frac{200}{3} \mathrm{H}_{0} \zeta_{3}-\frac{229}{4} \mathrm{H}_{0} \zeta_{2}-6 \mathrm{H}_{0} \zeta_{2} \zeta_{3}+313 \mathrm{H}_{2} \zeta_{4}-44 \mathrm{H}_{2} \zeta_{3}+320 \mathrm{H}_{3} \zeta_{3}+226 \mathrm{H}_{4}+12 \mathrm{H}_{4} \zeta_{2} \\
& +\frac{640}{3} \mathrm{H}_{5}+40 \mathrm{H}_{6}-96 \mathrm{H}_{-5,0}-90 \mathrm{H}_{-4,0}+120 \mathrm{H}_{-4,2}-384 \mathrm{H}_{-3,-1} \zeta_{2}+36 \mathrm{H}_{-3,0}+156 \mathrm{H}_{-3,0} \zeta_{2} \\
& -96 \mathrm{H}_{-3,2}+64 \mathrm{H}_{-3,3}-192 \mathrm{H}_{-2,-2} \zeta_{2}+140 \mathrm{H}_{-2,0} \zeta_{3}+18 \mathrm{H}_{-2,0} \zeta_{2}-96 \mathrm{H}_{-2,3}-24 \mathrm{H}_{-2,4} \\
& +\frac{11}{4} \mathrm{H}_{0,0}+34 \mathrm{H}_{0,0} \zeta_{4}-\frac{356}{3} \mathrm{H}_{0,0} \zeta_{3}-\frac{464}{3} \mathrm{H}_{0,0} \zeta_{2}-272 \mathrm{H}_{1,-3} \zeta_{2}+313 \mathrm{H}_{1,0} \zeta_{4}-44 \mathrm{H}_{1,0} \zeta_{3} \\
& +320 \mathrm{H}_{1,2} \zeta_{3}-232 \mathrm{H}_{1,4}+64 \mathrm{H}_{1,5}-136 \mathrm{H}_{2,-2} \zeta_{2}+352 \mathrm{H}_{2,0} \zeta_{3}+\frac{52}{3} \mathrm{H}_{2,0} \zeta_{2}+320 \mathrm{H}_{2,1} \zeta_{3} \\
& -\frac{232}{3} \mathrm{H}_{2,3}+112 \mathrm{H}_{2,4}+\frac{226}{3} \mathrm{H}_{3,0}+4 \mathrm{H}_{3,0} \zeta_{2}+48 \mathrm{H}_{3,2}+64 \mathrm{H}_{3,3}+\frac{508}{3} \mathrm{H}_{4,0}+144 \mathrm{H}_{4,1} \\
& +32 \mathrm{H}_{4,2}+72 \mathrm{H}_{5,0}+128 \mathrm{H}_{5,1}+408 \mathrm{H}_{-4,-1,0}-192 \mathrm{H}_{-4,0,0}+360 \mathrm{H}_{-3,-2,0}-120 \mathrm{H}_{-3,-1,0} \\
& +160 \mathrm{H}_{-3,-1,2}-48 \mathrm{H}_{-3,0,0}+104 \mathrm{H}_{-3,2,0}+128 \mathrm{H}_{-3,2,1}+224 \mathrm{H}_{-2,-3,0}-60 \mathrm{H}_{-2,-2,0} \\
& +80 \mathrm{H}_{-2,-2,2}-192 \mathrm{H}_{-2,-1,0} \zeta_{2}+160 \mathrm{H}_{-2,-1,3}+36 \mathrm{H}_{-2,0,0}+128 \mathrm{H}_{-2,0,0} \zeta_{2}-48 \mathrm{H}_{-2,2,0} \\
& +64 \mathrm{H}_{-2,2,2}+56 \mathrm{H}_{-2,3,0}+128 \mathrm{H}_{-2,3,1}-\frac{597}{4} \mathrm{H}_{0,0,0}+120 \mathrm{H}_{0,0,0} \zeta_{3}-250 \mathrm{H}_{0,0,0} \zeta_{2}+120 \mathrm{H}_{1,-4,0} \\
& -240 \mathrm{H}_{1,-3,0}+320 \mathrm{H}_{1,-3,2}-136 \mathrm{H}_{1,-2,0} \zeta_{2}+320 \mathrm{H}_{1,-2,3}+384 \mathrm{H}_{1,0,0} \zeta_{3}+\frac{104}{3} \mathrm{H}_{1,0,0} \zeta_{2} \\
& +320 \mathrm{H}_{1,1,0} \zeta_{3}+192 \mathrm{H}_{1,1,4}+64 \mathrm{H}_{1,2,3}-\frac{232}{3} \mathrm{H}_{1,3,0}+128 \mathrm{H}_{1,3,2}+208 \mathrm{H}_{1,4,0}+384 \mathrm{H}_{1,4,1} \\
& +168 \mathrm{H}_{2,-3,0}-120 \mathrm{H}_{2,-2,0}+160 \mathrm{H}_{2,-2,2}+\frac{400}{3} \mathrm{H}_{2,0,0}+8 \mathrm{H}_{2,0,0} \zeta_{2}+64 \mathrm{H}_{2,1,3}+96 \mathrm{H}_{2,3,0} \\
& +128 \mathrm{H}_{2,3,1}+128 \mathrm{H}_{3,-2,0}+48 \mathrm{H}_{3,0,0}+48 \mathrm{H}_{3,1,0}-16 \mathrm{H}_{4,0,0}+32 \mathrm{H}_{4,1,0}-448 \mathrm{H}_{-3,-1,-1,0} \\
& +576 \mathrm{H}_{-3,-1,0,0}-224 \mathrm{H}_{-3,0,0,0}-224 \mathrm{H}_{-2,-2,-1,0}+376 \mathrm{H}_{-2,-2,0,0}-224 \mathrm{H}_{-2,-1,-2,0} \\
& -120 \mathrm{H}_{-2,-1,0,0}+80 \mathrm{H}_{-2,-1,2,0}+18 \mathrm{H}_{-2,0,0,0}+64 \mathrm{H}_{-2,2,0,0}+64 \mathrm{H}_{-2,2,1,0}+\frac{748}{3} \mathrm{H}_{0,0,0,0} \\
& -64 \mathrm{H}_{0,0,0,0} \zeta_{2}+96 \mathrm{H}_{1,-3,-1,0}+192 \mathrm{H}_{1,-3,0,0}+48 \mathrm{H}_{1,-2,-2,0}-240 \mathrm{H}_{1,-2,0,0}+160 \mathrm{H}_{1,-2,2,0} \\
& +400 \mathrm{H}_{1,0,0,0}+24 \mathrm{H}_{1,0,0,0} \zeta_{2}+256 \mathrm{H}_{1,1,-3,0}+64 \mathrm{H}_{1,1,3,0}+128 \mathrm{H}_{1,2,-2,0}-\frac{176}{3} \mathrm{H}_{1,2,0,0} \\
& -64 \mathrm{H}_{1,3,0,0}+128 \mathrm{H}_{1,3,1,0}+48 \mathrm{H}_{2,-2,-1,0}+184 \mathrm{H}_{2,-2,0,0}-8 \mathrm{H}_{2,0,0,0}+128 \mathrm{H}_{2,1,-2,0} \\
& -\frac{176}{3} \mathrm{H}_{2,1,0,0}-192 \mathrm{H}_{2,2,0,0}-176 \mathrm{H}_{3,0,0,0}-192 \mathrm{H}_{3,1,0,0}-448 \mathrm{H}_{-2,-1,-1,0,0}+456 \mathrm{H}_{-2,-1,0,0,0} \\
& -160 \mathrm{H}_{-2,0,0,0,0}+310 \mathrm{H}_{0,0,0,0,0}+96 \mathrm{H}_{1,-2,-1,0,0}+168 \mathrm{H}_{1,-2,0,0,0}+144 \mathrm{H}_{1,0,0,0,0} \\
& +256 \mathrm{H}_{1,1,-2,0,0}-176 \mathrm{H}_{1,1,0,0,0}-192 \mathrm{H}_{1,1,2,0,0}-416 \mathrm{H}_{1,2,0,0,0}-192 \mathrm{H}_{1,2,1,0,0}-296 \mathrm{H}_{2,0,0,0,0} \\
& -416 \mathrm{H}_{2,1,0,0,0}-192 \mathrm{H}_{2,1,1,0,0}+48 \mathrm{H}_{0,0,0,0,0,0}-200 \mathrm{H}_{1,0,0,0,0,0}-512 \mathrm{H}_{1,1,0,0,0,0}
\end{aligned}
$$




$$
\begin{aligned}
& \left.\left.-576 \mathrm{H}_{1,1,1,0,0,0}\right)\right\} \\
& +\frac{16}{3} C_{F}^{2} C_{A}^{2}\left\{( 1 + x ) \left(-708 \mathrm{H}_{-2} \zeta_{3}-164 \mathrm{H}_{-2} \zeta_{2}+120 \mathrm{H}_{3} \zeta_{3}+720 \mathrm{H}_{-2,-1} \zeta_{2}-336 \mathrm{H}_{-2,0}\right.\right. \\
& +204 \mathrm{H}_{-2,2}+720 \mathrm{H}_{-1,-2} \zeta_{2}-708 \mathrm{H}_{-1,0} \zeta_{3}-164 \mathrm{H}_{-1,0} \zeta_{2}+408 \mathrm{H}_{-1,3}+1368 \mathrm{H}_{-1,4}+60 \mathrm{H}_{2,0} \zeta_{3} \\
& -336 \mathrm{H}_{2,2}+24 \mathrm{H}_{2,2} \zeta_{2}+162 \mathrm{H}_{2,4}-672 \mathrm{H}_{3,1}+48 \mathrm{H}_{3,1} \zeta_{2}-144 \mathrm{H}_{3,2}+108 \mathrm{H}_{3,3}-432 \mathrm{H}_{4,1} \\
& -36 \mathrm{H}_{4,2}-144 \mathrm{H}_{5,1}+80 \mathrm{H}_{-2,-1,0}-624 \mathrm{H}_{-2,-1,2}+288 \mathrm{H}_{-2,2,1}-192 \mathrm{H}_{-1,-3,0}+80 \mathrm{H}_{-1,-2,0} \\
& -624 \mathrm{H}_{-1,-2,2}+720 \mathrm{H}_{-1,-1,0} \zeta_{2}-1248 \mathrm{H}_{-1,-1,3}-672 \mathrm{H}_{-1,0,0}-1104 \mathrm{H}_{-1,0,0} \zeta_{2}+204 \mathrm{H}_{-1,2,0} \\
& +288 \mathrm{H}_{-1,2,2}+744 \mathrm{H}_{-1,3,0}+576 \mathrm{H}_{-1,3,1}+96 \mathrm{H}_{2,-3,0}-36 \mathrm{H}_{2,0,0} \zeta_{2}-336 \mathrm{H}_{2,1,0}+24 \mathrm{H}_{2,1,0} \zeta_{2} \\
& +54 \mathrm{H}_{2,3,0}+96 \mathrm{H}_{3,-2,0}-144 \mathrm{H}_{3,1,0}-99 \mathrm{H}_{4,0,0}-36 \mathrm{H}_{4,1,0}+192 \mathrm{H}_{-2,-1,-1,0}+192 \mathrm{H}_{-1,-2,-1,0} \\
& -600 \mathrm{H}_{-1,-2,0,0}+192 \mathrm{H}_{-1,-1,-2,0}+160 \mathrm{H}_{-1,-1,0,0}-624 \mathrm{H}_{-1,-1,2,0}-78 \mathrm{H}_{-1,0,0,0} \\
& +288 \mathrm{H}_{-1,2,0,0}+288 \mathrm{H}_{-1,2,1,0}+96 \mathrm{H}_{2,-2,0,0}-42 \mathrm{H}_{2,2,0,0}-99 \mathrm{H}_{3,0,0,0}-84 \mathrm{H}_{3,1,0,0} \\
& \left.+384 \mathrm{H}_{-1,-1,-1,0,0}-1224 \mathrm{H}_{-1,-1,0,0,0}+768 \mathrm{H}_{-1,0,0,0,0}-72 \mathrm{H}_{2,0,0,0,0}-126 \mathrm{H}_{2,1,0,0,0}\right) \\
& +(1-x)\left(360 \mathrm{H}_{-4} \zeta_{2}+372 \mathrm{H}_{-3} \zeta_{3}-\frac{16997}{27} \mathrm{H}_{0}-\frac{9800}{9} \mathrm{H}_{2}-276 \mathrm{H}_{2} \zeta_{3}+40 \mathrm{H}_{2} \zeta_{2}-24 \mathrm{H}_{-5,0}\right. \\
& -480 \mathrm{H}_{-3,-1} \zeta_{2}+408 \mathrm{H}_{-3,0} \zeta_{2}-240 \mathrm{H}_{-2,-2} \zeta_{2}+186 \mathrm{H}_{-2,0} \zeta_{3}-324 \mathrm{H}_{-2,4}-\frac{9800}{9} \mathrm{H}_{1,0} \\
& -276 \mathrm{H}_{1,0} \zeta_{3}+40 \mathrm{H}_{1,0} \zeta_{2}-528 \mathrm{H}_{1,2}-96 \mathrm{H}_{1,2} \zeta_{2}-144 \mathrm{H}_{1,4}-528 \mathrm{H}_{2,1}-96 \mathrm{H}_{2,1} \zeta_{2}-48 \mathrm{H}_{2,3} \\
& +432 \mathrm{H}_{-3,-1,2}+48 \mathrm{H}_{-2,-3,0}+216 \mathrm{H}_{-2,-2,2}-240 \mathrm{H}_{-2,-1,0} \zeta_{2}+432 \mathrm{H}_{-2,-1,3}+288 \mathrm{H}_{-2,0,0} \zeta_{2} \\
& -108 \mathrm{H}_{-2,3,0}-192 \mathrm{H}_{1,-3,0}-\frac{2200}{3} \mathrm{H}_{1,0,0}-96 \mathrm{H}_{1,0,0} \zeta_{2}-528 \mathrm{H}_{1,1,0}-96 \mathrm{H}_{1,1,0} \zeta_{2}-48 \mathrm{H}_{1,3,0} \\
& -96 \mathrm{H}_{2,-2,0}-96 \mathrm{H}_{-3,-1,-1,0}-48 \mathrm{H}_{-2,-2,-1,0}+180 \mathrm{H}_{-2,-2,0,0}-48 \mathrm{H}_{-2,-1,-2,0} \\
& +216 \mathrm{H}_{-2,-1,2,0}-192 \mathrm{H}_{1,-2,0,0}+228 \mathrm{H}_{1,0,0,0}+144 \mathrm{H}_{1,2,0,0}+144 \mathrm{H}_{2,1,0,0}-96 \mathrm{H}_{-2,-1,-1,0,0} \\
& \left.+396 \mathrm{H}_{-2,-1,0,0,0}-192 \mathrm{H}_{-2,0,0,0,0}+384 \mathrm{H}_{1,0,0,0,0}+432 \mathrm{H}_{1,1,0,0,0}\right)+(10-346 x) \mathrm{H}_{-3,0} \\
& -(16+372 x) \mathrm{H}_{-2,0,0}-\left(\frac{87}{2}+\frac{21}{2} x\right) \mathrm{H}_{0,0} \zeta_{4}-(45+105 x) \mathrm{H}_{5,0}+\left(\frac{123}{2}+\frac{1001}{2} x\right) \mathrm{H}_{0} \zeta_{3} \\
& +(66+930 x) \mathrm{H}_{0,0} \zeta_{3}-(75-51 x) \mathrm{H}_{0} \zeta_{5}-(84-132 x) \mathrm{H}_{-3,2,0}+(90-174 x) \mathrm{H}_{0} \zeta_{2} \zeta_{3} \\
& +\left(\frac{207}{2}+\frac{1053}{2} x\right) \mathrm{H}_{0,0,0} \zeta_{2}-(108-156 x) \mathrm{H}_{0,0,0,0} \zeta_{2}+(108-36 x) \mathrm{H}_{4} \zeta_{2}-(117-75 x) \mathrm{H}_{2,0} \zeta_{2} \\
& -(117+405 x) \mathrm{H}_{3,0,0}+(120-24 x) \mathrm{H}_{-3,-2,0}+(121+197 x) \mathrm{H}_{0,0,0,0}-\left(\frac{755}{6}-\frac{11119}{6} x\right) \mathrm{H}_{0,0,0} \\
& +(135-165 x) \mathrm{H}_{6}+\left(\frac{273}{2}-\frac{975}{2} x\right) \mathrm{H}_{2,0,0,0}-(138-54 x) \mathrm{H}_{3} \zeta_{2}-(138+650 x) \mathrm{H}_{5} \\
& +(141+39 x) \mathrm{H}_{-4,0}-\left(\frac{1421}{8}-\frac{753}{8} x\right) \mathrm{H}_{0} \zeta_{4}+(216+72 x) \mathrm{H}_{-4,-1,0}-(234+150 x) \mathrm{H}_{-2,-2,0} \\
& -(246-174 x) \mathrm{H}_{-4,0,0}-\left(\frac{501}{2}+\frac{757}{2} x\right) \mathrm{H}_{4,0}-(252-396 x) \mathrm{H}_{-4,2}-(260+412 x) \mathrm{H}_{2,0,0} \\
& +(272+976 x) \mathrm{H}_{-3,2}-(276+108 x) \mathrm{H}_{-3,-1,0}+(280+632 x) \mathrm{H}_{-2,2,0}+(288+446 x) \mathrm{H}_{0,0} \zeta_{2}
\end{aligned}
$$




$$
\begin{aligned}
& +\left(\frac{974}{3}-\frac{2234}{3} x\right) \mathrm{H}_{0} \zeta_{2}-(330-258 x) \mathrm{H}_{-3,0,0,0}-(384-480 x) \mathrm{H}_{-3,3} \\
& +(408-216 x) \mathrm{H}_{-3,-1,0,0}-(410+1030 x) \mathrm{H}_{-3} \zeta_{2}-(432+1188 x) \mathrm{H}_{4}+(456+480 x) \mathrm{H}_{-3,0,0} \\
& -(480+732 x) \mathrm{H}_{3,0}-\left(\frac{1948}{3}-\frac{2452}{3} x\right) \mathrm{H}_{3}-(684+516 x) \mathrm{H}_{-2,-1,0,0}-\left(\frac{2074}{3}-\frac{2326}{3} x\right) \mathrm{H}_{2,0} \\
& +(728+1432 x) \mathrm{H}_{-2,3}+(735+873 x) \mathrm{H}_{-2,0,0,0}-(757+1067 x) \mathrm{H}_{-2,0} \zeta_{2} \\
& -\left(\frac{20899}{27}-\frac{51353}{27} x\right) \mathrm{H}_{0,0}-108 x \mathrm{H}_{0,0,0,0,0,0}-75 x \mathrm{H}_{0,0,0,0,0}-48 x \mathrm{H}_{3,0} \zeta_{2}+252 x \mathrm{H}_{0,0,0} \zeta_{3} \\
& +p_{\mathrm{qq}}(-x)\left(-648 \mathrm{H}_{-4} \zeta_{2}-1248 \mathrm{H}_{-3} \zeta_{3}+288 \mathrm{H}_{-3} \zeta_{2}+570 \mathrm{H}_{-2} \zeta_{4}-4 \mathrm{H}_{-2} \zeta_{3}+602 \mathrm{H}_{-2} \zeta_{2}\right. \\
& +129 \mathrm{H}_{0} \zeta_{5}-25 \mathrm{H}_{0} \zeta_{4}-\frac{1037}{2} \mathrm{H}_{0} \zeta_{3}+\frac{177}{4} \mathrm{H}_{0} \zeta_{2}-354 \mathrm{H}_{0} \zeta_{2} \zeta_{3}+36 \mathrm{H}_{3}-72 \mathrm{H}_{3} \zeta_{3}+36 \mathrm{H}_{3} \zeta_{2} \\
& +501 \mathrm{H}_{4}-144 \mathrm{H}_{4} \zeta_{2}+216 \mathrm{H}_{5}-360 \mathrm{H}_{6}-318 \mathrm{H}_{-4,0}+648 \mathrm{H}_{-4,2}+1152 \mathrm{H}_{-3,-1} \zeta_{2}-470 \mathrm{H}_{-3,0} \\
& -1272 \mathrm{H}_{-3,0} \zeta_{2}-200 \mathrm{H}_{-3,2}+1392 \mathrm{H}_{-3,3}+1152 \mathrm{H}_{-2,-2} \zeta_{2}+1248 \mathrm{H}_{-2,-1} \zeta_{3}-112 \mathrm{H}_{-2,-1} \zeta_{2} \\
& +\frac{249}{2} \mathrm{H}_{-2,0}-1056 \mathrm{H}_{-2,0} \zeta_{3}+316 \mathrm{H}_{-2,0} \zeta_{2}-334 \mathrm{H}_{-2,2}-256 \mathrm{H}_{-2,3}+1752 \mathrm{H}_{-2,4} \\
& +1152 \mathrm{H}_{-1,-3} \zeta_{2}+1248 \mathrm{H}_{-1,-2} \zeta_{3}-112 \mathrm{H}_{-1,-2} \zeta_{2}+570 \mathrm{H}_{-1,0} \zeta_{4}-4 \mathrm{H}_{-1,0} \zeta_{3}+602 \mathrm{H}_{-1,0} \zeta_{2} \\
& -668 \mathrm{H}_{-1,3}-168 \mathrm{H}_{-1,4}+1248 \mathrm{H}_{-1,5}-27 \mathrm{H}_{0,0}-222 \mathrm{H}_{0,0} \zeta_{4}-158 \mathrm{H}_{0,0} \zeta_{3}-542 \mathrm{H}_{0,0} \zeta_{2} \\
& +18 \mathrm{H}_{2,0}-36 \mathrm{H}_{2,0} \zeta_{3}+18 \mathrm{H}_{2,0} \zeta_{2}-96 \mathrm{H}_{2,2} \zeta_{2}+167 \mathrm{H}_{3,0}-144 \mathrm{H}_{3,0} \zeta_{2}-192 \mathrm{H}_{3,1} \zeta_{2}-88 \mathrm{H}_{3,2} \\
& -12 \mathrm{H}_{4,0}-264 \mathrm{H}_{4,1}-96 \mathrm{H}_{4,2}-264 \mathrm{H}_{5,0}-384 \mathrm{H}_{5,1}+324 \mathrm{H}_{-4,0,0}+176 \mathrm{H}_{-3,-1,0} \\
& -1152 \mathrm{H}_{-3,-1,2}-576 \mathrm{H}_{-3,0,0}+600 \mathrm{H}_{-3,2,0}+768 \mathrm{H}_{-3,2,1}+176 \mathrm{H}_{-2,-2,0}-1152 \mathrm{H}_{-2,-2,2} \\
& -1152 \mathrm{H}_{-2,-1,-1} \zeta_{2}+536 \mathrm{H}_{-2,-1,0}+1632 \mathrm{H}_{-2,-1,0} \zeta_{2}+112 \mathrm{H}_{-2,-1,2}-2112 \mathrm{H}_{-2,-1,3} \\
& -839 \mathrm{H}_{-2,0,0}-1416 \mathrm{H}_{-2,0,0} \zeta_{2}-56 \mathrm{H}_{-2,2,0}+176 \mathrm{H}_{-2,2,1}+384 \mathrm{H}_{-2,2,2}+1056 \mathrm{H}_{-2,3,0} \\
& +1152 \mathrm{H}_{-2,3,1}+176 \mathrm{H}_{-1,-3,0}-1152 \mathrm{H}_{-1,-3,2}-1152 \mathrm{H}_{-1,-2,-1} \zeta_{2}+536 \mathrm{H}_{-1,-2,0} \\
& +1632 \mathrm{H}_{-1,-2,0} \zeta_{2}+112 \mathrm{H}_{-1,-2,2}-2112 \mathrm{H}_{-1,-2,3}-1152 \mathrm{H}_{-1,-1,-2} \zeta_{2} \\
& +1248 \mathrm{H}_{-1,-1,0} \zeta_{3}-112 \mathrm{H}_{-1,-1,0} \zeta_{2}+224 \mathrm{H}_{-1,-1,3}-2880 \mathrm{H}_{-1,-1,4}+249 \mathrm{H}_{-1,0,0} \\
& -864 \mathrm{H}_{-1,0,0} \zeta_{3}+344 \mathrm{H}_{-1,0,0} \zeta_{2}-334 \mathrm{H}_{-1,2,0}+176 \mathrm{H}_{-1,2,2}+32 \mathrm{H}_{-1,3,0}+352 \mathrm{H}_{-1,3,1} \\
& +384 \mathrm{H}_{-1,3,2}+888 \mathrm{H}_{-1,4,0}+1152 \mathrm{H}_{-1,4,1}-\frac{639}{4} \mathrm{H}_{0,0,0}+288 \mathrm{H}_{0,0,0} \zeta_{3}-402 \mathrm{H}_{0,0,0} \zeta_{2} \\
& -96 \mathrm{H}_{2,0,0} \zeta_{2}-96 \mathrm{H}_{2,1,0} \zeta_{2}-44 \mathrm{H}_{3,0,0}-88 \mathrm{H}_{3,1,0}-60 \mathrm{H}_{4,0,0}-96 \mathrm{H}_{4,1,0}-576 \mathrm{H}_{-3,-1,0,0} \\
& +660 \mathrm{H}_{-3,0,0,0}-576 \mathrm{H}_{-2,-2,0,0}+1152 \mathrm{H}_{-2,-1,-1,2}+408 \mathrm{H}_{-2,-1,0,0}-960 \mathrm{H}_{-2,-1,2,0} \\
& -768 \mathrm{H}_{-2,-1,2,1}-750 \mathrm{H}_{-2,0,0,0}+360 \mathrm{H}_{-2,2,0,0}+384 \mathrm{H}_{-2,2,1,0}-576 \mathrm{H}_{-1,-3,0,0} \\
& +1152 \mathrm{H}_{-1,-2,-1,2}+408 \mathrm{H}_{-1,-2,0,0}-960 \mathrm{H}_{-1,-2,2,0}-768 \mathrm{H}_{-1,-2,2,1}+1152 \mathrm{H}_{-1,-1,-2,2} \\
& -1152 \mathrm{H}_{-1,-1,-1,0} \zeta_{2}+2304 \mathrm{H}_{-1,-1,-1,3}+1072 \mathrm{H}_{-1,-1,0,0}+2112 \mathrm{H}_{-1,-1,0,0} \zeta_{2}+112 \mathrm{H}_{-1,-1,2,0} \\
& -768 \mathrm{H}_{-1,-1,2,2}-1728 \mathrm{H}_{-1,-1,3,0}-1536 \mathrm{H}_{-1,-1,3,1}-1107 \mathrm{H}_{-1,0,0,0}-1080 \mathrm{H}_{-1,0,0,0} \zeta_{2} \\
& +88 \mathrm{H}_{-1,2,0,0}+176 \mathrm{H}_{-1,2,1,0}+336 \mathrm{H}_{-1,3,0,0}+384 \mathrm{H}_{-1,3,1,0}+350 \mathrm{H}_{0,0,0,0}+288 \mathrm{H}_{0,0,0,0} \zeta_{2} \\
& +36 \mathrm{H}_{3,0,0,0}+576 \mathrm{H}_{-2,-1,-1,0,0}-1200 \mathrm{H}_{-2,-1,0,0,0}+744 \mathrm{H}_{-2,0,0,0,0}+576 \mathrm{H}_{-1,-2,-1,0,0} \\
& -1200 \mathrm{H}_{-1,-2,0,0,0}+576 \mathrm{H}_{-1,-1,-2,0,0}+1152 \mathrm{H}_{-1,-1,-1,2,0}+696 \mathrm{H}_{-1,-1,0,0,0} \\
& -768 \mathrm{H}_{-1,-1,2,0,0}-768 \mathrm{H}_{-1,-1,2,1,0}-816 \mathrm{H}_{-1,0,0,0,0}-72 \mathrm{H}_{-1,2,0,0,0}+510 \mathrm{H}_{0,0,0,0,0}
\end{aligned}
$$




$$
\begin{aligned}
& \left.+1728 \mathrm{H}_{-1,-1,-1,0,0,0}-1344 \mathrm{H}_{-1,-1,0,0,0,0}+360 \mathrm{H}_{-1,0,0,0,0,0}-72 \mathrm{H}_{0,0,0,0,0,0}\right) \\
& +p_{\mathrm{qq}}(x)\left(72 \mathrm{H}_{-4} \zeta_{2}-168 \mathrm{H}_{-3} \zeta_{3}-108 \mathrm{H}_{-3} \zeta_{2}+\frac{10537}{144} \mathrm{H}_{0}-9 \mathrm{H}_{0} \zeta_{5}-\frac{163}{2} \mathrm{H}_{0} \zeta_{4}+\frac{242}{3} \mathrm{H}_{0} \zeta_{3}\right. \\
& +\frac{4879}{36} \mathrm{H}_{0} \zeta_{2}+66 \mathrm{H}_{0} \zeta_{2} \zeta_{3}-\frac{11104}{27} \mathrm{H}_{2}-642 \mathrm{H}_{2} \zeta_{4}-4 \mathrm{H}_{2} \zeta_{3}+268 \mathrm{H}_{2} \zeta_{2}-\frac{3560}{9} \mathrm{H}_{3}-480 \mathrm{H}_{3} \zeta_{3} \\
& +88 \mathrm{H}_{3} \zeta_{2}-\frac{947}{2} \mathrm{H}_{4}-264 \mathrm{H}_{5}+162 \mathrm{H}_{-4,0}-216 \mathrm{H}_{-4,2}+288 \mathrm{H}_{-3,-1} \zeta_{2}-54 \mathrm{H}_{-3,0}-24 \mathrm{H}_{-3,0} \zeta_{2} \\
& +144 \mathrm{H}_{-3,2}-192 \mathrm{H}_{-3,3}+144 \mathrm{H}_{-2,-2} \zeta_{2}-84 \mathrm{H}_{-2,0} \zeta_{3}-54 \mathrm{H}_{-2,0} \zeta_{2}+144 \mathrm{H}_{-2,3}-72 \mathrm{H}_{-2,4} \\
& -\frac{23959}{108} \mathrm{H}_{0,0}-306 \mathrm{H}_{0,0} \zeta_{4}+134 \mathrm{H}_{0,0} \zeta_{3}+\frac{1571}{3} \mathrm{H}_{0,0} \zeta_{2}+384 \mathrm{H}_{1,-3} \zeta_{2}-\frac{11104}{27} \mathrm{H}_{1,0} \\
& -642 \mathrm{H}_{1,0} \zeta_{4}-4 \mathrm{H}_{1,0} \zeta_{3}+268 \mathrm{H}_{1,0} \zeta_{2}-480 \mathrm{H}_{1,2} \zeta_{3}+348 \mathrm{H}_{1,4}-192 \mathrm{H}_{1,5}+192 \mathrm{H}_{2,-2} \zeta_{2} \\
& -\frac{3560}{9} \mathrm{H}_{2,0}-528 \mathrm{H}_{2,0} \zeta_{3}+44 \mathrm{H}_{2,0} \zeta_{2}-480 \mathrm{H}_{2,1} \zeta_{3}+116 \mathrm{H}_{2,3}-192 \mathrm{H}_{2,4}-\frac{477}{2} \mathrm{H}_{3,0}-44 \mathrm{H}_{3,2} \\
& -96 \mathrm{H}_{3,3}-198 \mathrm{H}_{4,0}-132 \mathrm{H}_{4,1}-48 \mathrm{H}_{4,2}-96 \mathrm{H}_{5,0}-192 \mathrm{H}_{5,1}-288 \mathrm{H}_{-4,-1,0}+36 \mathrm{H}_{-4,0,0} \\
& -288 \mathrm{H}_{-3,-2,0}+72 \mathrm{H}_{-3,-1,0}-96 \mathrm{H}_{-3,-1,2}+144 \mathrm{H}_{-3,0,0}-168 \mathrm{H}_{-3,2,0}-192 \mathrm{H}_{-3,2,1} \\
& -192 \mathrm{H}_{-2,-3,0}+36 \mathrm{H}_{-2,-2,0}-48 \mathrm{H}_{-2,-2,2}+144 \mathrm{H}_{-2,-1,0} \zeta_{2}-96 \mathrm{H}_{-2,-1,3}-54 \mathrm{H}_{-2,0,0} \\
& -48 \mathrm{H}_{-2,0,0} \zeta_{2}+72 \mathrm{H}_{-2,2,0}-96 \mathrm{H}_{-2,2,2}-120 \mathrm{H}_{-2,3,0}-192 \mathrm{H}_{-2,3,1}-\frac{1733}{6} \mathrm{H}_{0,0,0}-288 \mathrm{H}_{0,0,0} \zeta_{3} \\
& +438 \mathrm{H}_{0,0,0} \zeta_{2}-288 \mathrm{H}_{1,-4,0}+288 \mathrm{H}_{1,-3,0}-384 \mathrm{H}_{1,-3,2}+192 \mathrm{H}_{1,-2,0} \zeta_{2}-384 \mathrm{H}_{1,-2,3} \\
& -\frac{3560}{9} \mathrm{H}_{1,0,0}-576 \mathrm{H}_{1,0,0} \zeta_{3}-480 \mathrm{H}_{1,1,0} \zeta_{3}-288 \mathrm{H}_{1,1,4}-96 \mathrm{H}_{1,2,3}+116 \mathrm{H}_{1,3,0}-192 \mathrm{H}_{1,3,2} \\
& -336 \mathrm{H}_{1,4,0}-576 \mathrm{H}_{1,4,1}-288 \mathrm{H}_{2,-3,0}+144 \mathrm{H}_{2,-2,0}-192 \mathrm{H}_{2,-2,2}-\frac{609}{2} \mathrm{H}_{2,0,0}-96 \mathrm{H}_{2,1,3} \\
& -144 \mathrm{H}_{2,3,0}-192 \mathrm{H}_{2,3,1}-192 \mathrm{H}_{3,-2,0}-72 \mathrm{H}_{3,0,0}-44 \mathrm{H}_{3,1,0}+60 \mathrm{H}_{4,0,0}-48 \mathrm{H}_{4,1,0} \\
& +384 \mathrm{H}_{-3,-1,-1,0}-432 \mathrm{H}_{-3,-1,0,0}+84 \mathrm{H}_{-3,0,0,0}+192 \mathrm{H}_{-2,-2,-1,0}-312 \mathrm{H}_{-2,-2,0,0} \\
& +192 \mathrm{H}_{-2,-1,-2,0}+72 \mathrm{H}_{-2,-1,0,0}-48 \mathrm{H}_{-2,-1,2,0}+54 \mathrm{H}_{-2,0,0,0}-96 \mathrm{H}_{-2,2,0,0}-96 \mathrm{H}_{-2,2,1,0} \\
& -592 \mathrm{H}_{0,0,0,0}-384 \mathrm{H}_{1,-3,0,0}+288 \mathrm{H}_{1,-2,0,0}-192 \mathrm{H}_{1,-2,2,0}-\frac{1343}{2} \mathrm{H}_{1,0,0,0}-384 \mathrm{H}_{1,1,-3,0} \\
& -96 \mathrm{H}_{1,1,3,0}-192 \mathrm{H}_{1,2,-2,0}+60 \mathrm{H}_{1,2,0,0}+96 \mathrm{H}_{1,3,0,0}-192 \mathrm{H}_{1,3,1,0}-288 \mathrm{H}_{2,-2,0,0}-26 \mathrm{H}_{2,0,0,0} \\
& -192 \mathrm{H}_{2,1,-2,0}+60 \mathrm{H}_{2,1,0,0}+288 \mathrm{H}_{2,2,0,0}+300 \mathrm{H}_{3,0,0,0}+288 \mathrm{H}_{3,1,0,0}+384 \mathrm{H}_{-2,-1,-1,0,0} \\
& -360 \mathrm{H}_{-2,-1,0,0,0}+96 \mathrm{H}_{-2,0,0,0,0}-510 \mathrm{H}_{0,0,0,0,0}-288 \mathrm{H}_{1,-2,0,0,0}-200 \mathrm{H}_{1,0,0,0,0} \\
& -384 \mathrm{H}_{1,1,-2,0,0}+180 \mathrm{H}_{1,1,0,0,0}+288 \mathrm{H}_{1,1,2,0,0}+624 \mathrm{H}_{1,2,0,0,0}+288 \mathrm{H}_{1,2,1,0,0}+456 \mathrm{H}_{2,0,0,0,0} \\
& +624 \mathrm{H}_{2,1,0,0,0}+288 \mathrm{H}_{2,1,1,0,0}+72 \mathrm{H}_{0,0,0,0,0,0}+360 \mathrm{H}_{1,0,0,0,0,0}+768 \mathrm{H}_{1,1,0,0,0,0} \\
& \left.\left.+864 \mathrm{H}_{1,1,1,0,0,0}\right)\right\} \\
& +\frac{16}{3} C_{F}^{3} n_{f}\left\{( 1 + x ) \left(32 \mathrm{H}_{-2} \zeta_{2}-60 \mathrm{H}_{4}-120 \mathrm{H}_{-2,0}+32 \mathrm{H}_{-1,0} \zeta_{2}+40 \mathrm{H}_{0,0} \zeta_{3}-32 \mathrm{H}_{2,2}-56 \mathrm{H}_{3,0}\right.\right. \\
& -64 \mathrm{H}_{3,1}+64 \mathrm{H}_{-2,-1,0}+64 \mathrm{H}_{-1,-2,0}-240 \mathrm{H}_{-1,0,0}-36 \mathrm{H}_{2,0,0}-32 \mathrm{H}_{2,1,0}-16 \mathrm{H}_{3,0,0} \\
& \left.+128 \mathrm{H}_{-1,-1,0,0}-192 \mathrm{H}_{-1,0,0,0}-24 \mathrm{H}_{2,0,0,0}\right)+(1-x)\left(32 \mathrm{H}_{-3} \zeta_{2}+\frac{35}{2} \mathrm{H}_{0}-20 \mathrm{H}_{2}+32 \mathrm{H}_{5}\right. \\
& +48 \mathrm{H}_{-4,0}-32 \mathrm{H}_{-3,2}+16 \mathrm{H}_{-2,0} \zeta_{2}-32 \mathrm{H}_{-2,3}-20 \mathrm{H}_{1,0}-64 \mathrm{H}_{1,2}-64 \mathrm{H}_{2,1}+8 \mathrm{H}_{4,0}+48 \mathrm{H}_{-3,0,0}
\end{aligned}
$$




$$
\begin{aligned}
& \left.-16 \mathrm{H}_{-2,2,0}-72 \mathrm{H}_{1,0,0}-64 \mathrm{H}_{1,1,0}+24 \mathrm{H}_{-2,0,0,0}\right)-(4+28 x) \mathrm{H}_{0} \zeta_{3}-(12-340 x) \mathrm{H}_{0,0,0,0} \\
& -(16+176 x) \mathrm{H}_{-3,0}-\left(\frac{39}{2}-\frac{825}{2} x\right) \mathrm{H}_{0,0,0}+(20+28 x) \mathrm{H}_{0} \zeta_{4}-\left(\frac{51}{2}-\frac{29}{2} x\right) \mathrm{H}_{0,0} \\
& -(27+123 x) \mathrm{H}_{0} \zeta_{2}+(32-48 x) \mathrm{H}_{0,0} \zeta_{2}-(42-102 x) \mathrm{H}_{3}-(57-87 x) \mathrm{H}_{2,0} \\
& -(80+240 x) \mathrm{H}_{-2,0,0}+120 x \mathrm{H}_{0,0,0,0,0}+p_{\mathrm{qq}}(-x)\left(-80 \mathrm{H}_{-2} \zeta_{3}+184 \mathrm{H}_{-2} \zeta_{2}-5 \mathrm{H}_{0} \zeta_{4}-158 \mathrm{H}_{0} \zeta_{3}\right. \\
& +21 \mathrm{H}_{0} \zeta_{2}+156 \mathrm{H}_{4}-96 \mathrm{H}_{-4,0}-136 \mathrm{H}_{-3,0}+32 \mathrm{H}_{-3,2}+64 \mathrm{H}_{-2,-1} \zeta_{2}+42 \mathrm{H}_{-2,0}-16 \mathrm{H}_{-2,0} \zeta_{2} \\
& -104 \mathrm{H}_{-2,2}+64 \mathrm{H}_{-2,3}+64 \mathrm{H}_{-1,-2} \zeta_{2}-80 \mathrm{H}_{-1,0} \zeta_{3}+184 \mathrm{H}_{-1,0} \zeta_{2}-208 \mathrm{H}_{-1,3}+96 \mathrm{H}_{-1,4} \\
& +8 \mathrm{H}_{0,0} \zeta_{3}-172 \mathrm{H}_{0,0} \zeta_{2}+52 \mathrm{H}_{3,0}-32 \mathrm{H}_{3,2}-24 \mathrm{H}_{4,0}-96 \mathrm{H}_{4,1}+64 \mathrm{H}_{-3,-1,0}-144 \mathrm{H}_{-3,0,0} \\
& +64 \mathrm{H}_{-2,-2,0}+160 \mathrm{H}_{-2,-1,0}-64 \mathrm{H}_{-2,-1,2}-244 \mathrm{H}_{-2,0,0}+32 \mathrm{H}_{-2,2,0}+64 \mathrm{H}_{-2,2,1}+64 \mathrm{H}_{-1,-3,0} \\
& +160 \mathrm{H}_{-1,-2,0}-64 \mathrm{H}_{-1,-2,2}+64 \mathrm{H}_{-1,-1,0} \zeta_{2}-128 \mathrm{H}_{-1,-1,3}+84 \mathrm{H}_{-1,0,0}-32 \mathrm{H}_{-1,0,0} \zeta_{2} \\
& -104 \mathrm{H}_{-1,2,0}+64 \mathrm{H}_{-1,2,2}+64 \mathrm{H}_{-1,3,0}+128 \mathrm{H}_{-1,3,1}-63 \mathrm{H}_{0,0,0}-48 \mathrm{H}_{0,0,0} \zeta_{2}-16 \mathrm{H}_{3,0,0} \\
& -32 \mathrm{H}_{3,1,0}+96 \mathrm{H}_{-2,-1,0,0}-168 \mathrm{H}_{-2,0,0,0}+96 \mathrm{H}_{-1,-2,0,0}+320 \mathrm{H}_{-1,-1,0,0}-64 \mathrm{H}_{-1,-1,2,0} \\
& -324 \mathrm{H}_{-1,0,0,0}+32 \mathrm{H}_{-1,2,0,0}+64 \mathrm{H}_{-1,2,1,0}+112 \mathrm{H}_{0,0,0,0}+96 \mathrm{H}_{-1,-1,0,0,0}-192 \mathrm{H}_{-1,0,0,0,0} \\
& \left.+120 \mathrm{H}_{0,0,0,0,0}\right)+p_{\mathrm{qq}}(x)\left(-\frac{313}{8} \mathrm{H}_{0}-15 \mathrm{H}_{0} \zeta_{4}+56 \mathrm{H}_{0} \zeta_{3}+15 \mathrm{H}_{0} \zeta_{2}+55 \mathrm{H}_{2}-96 \mathrm{H}_{2} \zeta_{3}+12 \mathrm{H}_{3}\right. \\
& -120 \mathrm{H}_{4}-64 \mathrm{H}_{5}+\frac{89}{2} \mathrm{H}_{0,0}-88 \mathrm{H}_{0,0} \zeta_{3}+92 \mathrm{H}_{0,0} \zeta_{2}+55 \mathrm{H}_{1,0}-96 \mathrm{H}_{1,0} \zeta_{3}+48 \mathrm{H}_{1,4}+12 \mathrm{H}_{2,0} \\
& -16 \mathrm{H}_{2,0} \zeta_{2}+16 \mathrm{H}_{2,3}-40 \mathrm{H}_{3,0}-40 \mathrm{H}_{4,0}+81 \mathrm{H}_{0,0,0}+48 \mathrm{H}_{0,0,0} \zeta_{2}+12 \mathrm{H}_{1,0,0}-32 \mathrm{H}_{1,0,0} \zeta_{2} \\
& +16 \mathrm{H}_{1,3,0}-52 \mathrm{H}_{2,0,0}-112 \mathrm{H}_{0,0,0,0}-156 \mathrm{H}_{1,0,0,0}+32 \mathrm{H}_{1,2,0,0}+24 \mathrm{H}_{2,0,0,0}+32 \mathrm{H}_{2,1,0,0} \\
& \left.\left.-120 \mathrm{H}_{0,0,0,0,0}+96 \mathrm{H}_{1,1,0,0,0}\right)\right\} \\
& +\frac{16}{3} C_{F}^{2} C_{A} n_{f}\left\{( 1 + x ) \left(-16 \mathrm{H}_{-2} \zeta_{2}+36 \mathrm{H}_{4}+60 \mathrm{H}_{-2,0}-16 \mathrm{H}_{-1,0} \zeta_{2}-36 \mathrm{H}_{0,0} \zeta_{3}+24 \mathrm{H}_{2,2}\right.\right. \\
& +36 \mathrm{H}_{3,0}+48 \mathrm{H}_{3,1}-32 \mathrm{H}_{-2,-1,0}-32 \mathrm{H}_{-1,-2,0}+120 \mathrm{H}_{-1,0,0}+18 \mathrm{H}_{0,0,0} \zeta_{2}+24 \mathrm{H}_{2,1,0}+12 \mathrm{H}_{3,0,0} \\
& \left.-64 \mathrm{H}_{-1,-1,0,0}+96 \mathrm{H}_{-1,0,0,0}+18 \mathrm{H}_{2,0,0,0}\right)+(1-x)\left(-16 \mathrm{H}_{-3} \zeta_{2}+\frac{9161}{54} \mathrm{H}_{0}+\frac{1846}{9} \mathrm{H}_{2}\right. \\
& -16 \mathrm{H}_{2} \zeta_{2}-24 \mathrm{H}_{-4,0}+16 \mathrm{H}_{-3,2}-8 \mathrm{H}_{-2,0} \zeta_{2}+16 \mathrm{H}_{-2,3}+\frac{1846}{9} \mathrm{H}_{1,0}-16 \mathrm{H}_{1,0} \zeta_{2}+48 \mathrm{H}_{1,2} \\
& \left.+48 \mathrm{H}_{2,1}-24 \mathrm{H}_{-3,0,0}+8 \mathrm{H}_{-2,2,0}+\frac{344}{3} \mathrm{H}_{1,0,0}+48 \mathrm{H}_{1,1,0}-12 \mathrm{H}_{-2,0,0,0}-12 \mathrm{H}_{1,0,0,0}\right) \\
& -(6-2 x) \mathrm{H}_{4,0}+(8+88 x) \mathrm{H}_{-3,0}-(14+18 x) \mathrm{H}_{0} \zeta_{4}+(18-10 x) \mathrm{H}_{0} \zeta_{3}+(20+28 x) \mathrm{H}_{2,0,0} \\
& -(24-64 x) \mathrm{H}_{0,0} \zeta_{2}-(24-8 x) \mathrm{H}_{5}+\left(\frac{77}{3}-\frac{1276}{3} x\right) \mathrm{H}_{0,0,0}+(40+120 x) \mathrm{H}_{-2,0,0} \\
& -(44+204 x) \mathrm{H}_{0,0,0,0}-\left(\frac{145}{3}-\frac{379}{3} x\right) \mathrm{H}_{0} \zeta_{2}+\left(\frac{290}{3}-\frac{398}{3} x\right) \mathrm{H}_{3}+\left(\frac{317}{3}-\frac{371}{3} x\right) \mathrm{H}_{2,0} \\
& +\left(\frac{6623}{27}-\frac{8821}{27} x\right) \mathrm{H}_{0,0}-60 x \mathrm{H}_{0,0,0,0,0}+p_{\mathrm{qq}}(-x)\left(40 \mathrm{H}_{-2} \zeta_{3}-92 \mathrm{H}_{-2} \zeta_{2}+\frac{5}{2} \mathrm{H}_{0} \zeta_{4}+79 \mathrm{H}_{0} \zeta_{3}\right. \\
& -\frac{21}{2} \mathrm{H}_{0} \zeta_{2}-78 \mathrm{H}_{4}+48 \mathrm{H}_{-4,0}+68 \mathrm{H}_{-3,0}-16 \mathrm{H}_{-3,2}-32 \mathrm{H}_{-2,-1} \zeta_{2}-21 \mathrm{H}_{-2,0}+8 \mathrm{H}_{-2,0} \zeta_{2} \\
& +52 \mathrm{H}_{-2,2}-32 \mathrm{H}_{-2,3}-32 \mathrm{H}_{-1,-2} \zeta_{2}+40 \mathrm{H}_{-1,0} \zeta_{3}-92 \mathrm{H}_{-1,0} \zeta_{2}+104 \mathrm{H}_{-1,3}-48 \mathrm{H}_{-1,4}
\end{aligned}
$$




$$
\begin{aligned}
&-4 \mathrm{H}_{0,0} \zeta_{3}+86 \mathrm{H}_{0,0} \zeta_{2}-26 \mathrm{H}_{3,0}+16 \mathrm{H}_{3,2}+12 \mathrm{H}_{4,0}+48 \mathrm{H}_{4,1}-32 \mathrm{H}_{-3,-1,0}+72 \mathrm{H}_{-3,0,0} \\
&-32 \mathrm{H}_{-2,-2,0}-80 \mathrm{H}_{-2,-1,0}+32 \mathrm{H}_{-2,-1,2}+122 \mathrm{H}_{-2,0,0}-16 \mathrm{H}_{-2,2,0}-32 \mathrm{H}_{-2,2,1}-32 \mathrm{H}_{-1,-3,0} \\
&-80 \mathrm{H}_{-1,-2,0}+32 \mathrm{H}_{-1,-2,2}-32 \mathrm{H}_{-1,-1,0} \zeta_{2}+64 \mathrm{H}_{-1,-1,3}-42 \mathrm{H}_{-1,0,0}+16 \mathrm{H}_{-1,0,0} \zeta_{2} \\
&+52 \mathrm{H}_{-1,2,0}-32 \mathrm{H}_{-1,2,2}-32 \mathrm{H}_{-1,3,0}-64 \mathrm{H}_{-1,3,1}+\frac{63}{2} \mathrm{H}_{0,0,0}+24 \mathrm{H}_{0,0,0} \zeta_{2}+8 \mathrm{H}_{3,0,0}+16 \mathrm{H}_{3,1,0} \\
&-48 \mathrm{H}_{-2,-1,0,0}+84 \mathrm{H}_{-2,0,0,0}-48 \mathrm{H}_{-1,-2,0,0}-160 \mathrm{H}_{-1,-1,0,0}+32 \mathrm{H}_{-1,-1,2,0}+162 \mathrm{H}_{-1,0,0,0} \\
&\left.-16 \mathrm{H}_{-1,2,0,0}-32 \mathrm{H}_{-1,2,1,0}-56 \mathrm{H}_{0,0,0,0}-48 \mathrm{H}_{-1,-1,0,0,0}+96 \mathrm{H}_{-1,0,0,0,0}-60 \mathrm{H}_{0,0,0,0,0}\right) \\
&+p_{\mathrm{qq}}(x)\left(-\frac{239}{36} \mathrm{H}_{0}+\frac{91}{2} \mathrm{H}_{0} \zeta_{4}-\frac{121}{3} \mathrm{H}_{0} \zeta_{3}-\frac{967}{18} \mathrm{H}_{0} \zeta_{2}+\frac{2594}{27} \mathrm{H}_{2}+88 \mathrm{H}_{2} \zeta_{3}-40 \mathrm{H}_{2} \zeta_{2}\right. \\
&+\frac{1156}{9} \mathrm{H}_{3}-16 \mathrm{H}_{3} \zeta_{2}+95 \mathrm{H}_{4}+48 \mathrm{H}_{5}+\frac{965}{27} \mathrm{H}_{0,0}+76 \mathrm{H}_{0,0} \zeta_{3}-\frac{292}{3} \mathrm{H}_{0,0} \zeta_{2}+\frac{2594}{27} \mathrm{H}_{1,0} \\
&+88 \mathrm{H}_{1,0} \zeta_{3}-40 \mathrm{H}_{1,0} \zeta_{2}-24 \mathrm{H}_{1,4}+\frac{1156}{9} \mathrm{H}_{2,0}-8 \mathrm{H}_{2,0} \zeta_{2}-8 \mathrm{H}_{2,3}+61 \mathrm{H}_{3,0}+8 \mathrm{H}_{3,2}+36 \mathrm{H}_{4,0} \\
&+24 \mathrm{H}_{4,1}+\frac{769}{6} \mathrm{H}_{0,0,0}-60 \mathrm{H}_{0,0,0} \zeta_{2}+\frac{1156}{9} \mathrm{H}_{1,0,0}-8 \mathrm{H}_{1,3,0}+73 \mathrm{H}_{2,0,0}+8 \mathrm{H}_{3,1,0}+144 \mathrm{H}_{0,0,0,0} \\
&\left.\left.+131 \mathrm{H}_{1,0,0,0}-24 \mathrm{H}_{1,2,0,0}-28 \mathrm{H}_{2,0,0,0}-24 \mathrm{H}_{2,1,0,0}+60 \mathrm{H}_{0,0,0,0,0}-16 \mathrm{H}_{1,0,0,0,0}-72 \mathrm{H}_{1,1,0,0,0}\right)\right\} \\
&+ \frac{16}{81} C_{F}^{2} n_{f}^{2}\left\{108(1+x) \mathrm{H}_{0,0,0,0}+(1-x)\left(-260 \mathrm{H}_{0}+72 \mathrm{H}_{0} \zeta_{2}-276 \mathrm{H}_{2}-144 \mathrm{H}_{3}-276 \mathrm{H}_{1,0}\right.\right. \\
&\left.-144 \mathrm{H}_{2,0}-144 \mathrm{H}_{1,0,0}\right)-(90-450 x) \mathrm{H}_{0,0,0}-(466-398 x) \mathrm{H}_{0,0}+p_{\mathrm{qq}}(x)\left(-\frac{159}{4} \mathrm{H}_{0}-36 \mathrm{H}_{0} \zeta_{3}\right. \\
&+120 \mathrm{H}_{0} \zeta_{2}-76 \mathrm{H}_{2}-240 \mathrm{H}_{3}-108 \mathrm{H}_{4}+23 \mathrm{H}_{0,0}+72 \mathrm{H}_{0,0} \zeta_{2}-76 \mathrm{H}_{1,0}-240 \mathrm{H}_{2,0}-108 \mathrm{H}_{3,0} \\
&\left.\left.-279 \mathrm{H}_{0,0,0}-240 \mathrm{H}_{1,0,0}-108 \mathrm{H}_{2,0,0}-216 \mathrm{H}_{0,0,0,0}-108 \mathrm{H}_{1,0,0,0}\right)\right\} . \\
&
\end{aligned}
$$

The most compact representation of $\delta P^{(3)-}(x)$ is via its difference to $\delta P^{(3)+}(x)$,

$$
\begin{aligned}
& \delta P^{(3)+}(x)-\delta P^{(3)-}(x)= \\
& 16 C_{F}^{3}\left(C_{A}-2 C_{F}\right)\left\{( 1 + x ) \left(-208 \mathrm{H}_{-2} \zeta_{3}+\frac{40}{3} \mathrm{H}_{-2} \zeta_{2}+192 \mathrm{H}_{-2,-1} \zeta_{2}-376 \mathrm{H}_{-2,0}+96 \mathrm{H}_{-2,2}\right.\right. \\
&+192 \mathrm{H}_{-1,-2} \zeta_{2}-208 \mathrm{H}_{-1,0} \zeta_{3}+\frac{40}{3} \mathrm{H}_{-1,0} \zeta_{2}+192 \mathrm{H}_{-1,3}+480 \mathrm{H}_{-1,4}-\frac{400}{3} \mathrm{H}_{2,2}-\frac{800}{3} \mathrm{H}_{3,1} \\
&+\frac{656}{3} \mathrm{H}_{-2,-1,0}-192 \mathrm{H}_{-2,-1,2}+128 \mathrm{H}_{-2,2,1}+\frac{656}{3} \mathrm{H}_{-1,-2,0}-192 \mathrm{H}_{-1,-2,2}+192 \mathrm{H}_{-1,-1,0} \zeta_{2} \\
&-384 \mathrm{H}_{-1,-1,3}-752 \mathrm{H}_{-1,0,0}-320 \mathrm{H}_{-1,0,0} \zeta_{2}+96 \mathrm{H}_{-1,2,0}+128 \mathrm{H}_{-1,2,2}+288 \mathrm{H}_{-1,3,0} \\
&+256 \mathrm{H}_{-1,3,1}-\frac{224}{3} \mathrm{H}_{2,0,0}-\frac{400}{3} \mathrm{H}_{2,1,0}-96 \mathrm{H}_{-1,-2,0,0}+\frac{1312}{3} \mathrm{H}_{-1,-1,0,0}-192 \mathrm{H}_{-1,-1,2,0} \\
&\left.-536 \mathrm{H}_{-1,0,0,0}+128 \mathrm{H}_{-1,2,0,0}+128 \mathrm{H}_{-1,2,1,0}-288 \mathrm{H}_{-1,-1,0,0,0}+384 \mathrm{H}_{-1,0,0,0,0}\right) \\
&+(1-x)\left(192 \mathrm{H}_{-4} \zeta_{2}+208 \mathrm{H}_{-3} \zeta_{3}+347 \mathrm{H}_{0}-206 \mathrm{H}_{0} \zeta_{5}+64 \mathrm{H}_{0} \zeta_{2} \zeta_{3}-\frac{452}{3} \mathrm{H}_{2}-24 \mathrm{H}_{2} \zeta_{3}\right. \\
&-60 \mathrm{H}_{2} \zeta_{2}+120 \mathrm{H}_{6}-128 \mathrm{H}_{-5,0}-144 \mathrm{H}_{-4,2}-256 \mathrm{H}_{-3,-1} \zeta_{2}+192 \mathrm{H}_{-3,0} \zeta_{2}-192 \mathrm{H}_{-3,3} \\
&-128 \mathrm{H}_{-2,-2} \zeta_{2}+104 \mathrm{H}_{-2,0} \zeta_{3}-144 \mathrm{H}_{-2,4}-152 \mathrm{H}_{0,0} \zeta_{4}-\frac{452}{3} \mathrm{H}_{1,0}-24 \mathrm{H}_{1,0} \zeta_{3}-60 \mathrm{H}_{1,0} \zeta_{2}
\end{aligned}
$$




$$
\begin{aligned}
& -\frac{800}{3} \mathrm{H}_{1,2}-32 \mathrm{H}_{1,2} \zeta_{2}-\frac{800}{3} \mathrm{H}_{2,1}-32 \mathrm{H}_{2,1} \zeta_{2}+24 \mathrm{H}_{5,0}+96 \mathrm{H}_{-4,-1,0}-184 \mathrm{H}_{-4,0,0} \\
& +96 \mathrm{H}_{-3,-2,0}-208 \mathrm{H}_{-3,-1,0}+192 \mathrm{H}_{-3,-1,2}-48 \mathrm{H}_{-3,2,0}+64 \mathrm{H}_{-2,-3,0}-104 \mathrm{H}_{-2,-2,0} \\
& +96 \mathrm{H}_{-2,-2,2}-128 \mathrm{H}_{-2,-1,0} \zeta_{2}+192 \mathrm{H}_{-2,-1,3}+128 \mathrm{H}_{-2,0,0} \zeta_{2}-48 \mathrm{H}_{-2,3,0}-192 \mathrm{H}_{0,0,0} \zeta_{3} \\
& -\frac{448}{3} \mathrm{H}_{1,0,0}-32 \mathrm{H}_{1,0,0} \zeta_{2}-\frac{800}{3} \mathrm{H}_{1,1,0}-32 \mathrm{H}_{1,1,0} \zeta_{2}-128 \mathrm{H}_{-3,-1,-1,0}+224 \mathrm{H}_{-3,-1,0,0} \\
& -216 \mathrm{H}_{-3,0,0,0}-64 \mathrm{H}_{-2,-2,-1,0}+144 \mathrm{H}_{-2,-2,0,0}-64 \mathrm{H}_{-2,-1,-2,0}+96 \mathrm{H}_{-2,-1,2,0} \\
& \left.-160 \mathrm{H}_{0,0,0,0} \zeta_{2}-128 \mathrm{H}_{-2,-1,-1,0,0}+240 \mathrm{H}_{-2,-1,0,0,0}-192 \mathrm{H}_{-2,0,0,0,0}+192 \mathrm{H}_{0,0,0,0,0,0}\right) \\
& +\left(12+\frac{3292}{3} x\right) \mathrm{H}_{0,0,0,0}-(32+96 x)\left(\mathrm{H}_{3,2}+\mathrm{H}_{3,0,0}+\mathrm{H}_{3,1,0}\right)-(40-24 x) \mathrm{H}_{2,0} \zeta_{2} \\
& +(42-186 x) \mathrm{H}_{0,0} \zeta_{2}-(48-16 x) \mathrm{H}_{3} \zeta_{2}+\left(\frac{148}{3}-\frac{2164}{3} x\right) \mathrm{H}_{-3,0}-\left(60-\frac{716}{3} x\right) \mathrm{H}_{3} \\
& -(96+288 x) \mathrm{H}_{4,1}-\left(\frac{314}{3}-194 x\right) \mathrm{H}_{2,0}-\left(\frac{344}{3}+\frac{664}{3} x\right) \mathrm{H}_{4,0}-\left(\frac{364}{3}+140 x\right) \mathrm{H}_{3,0} \\
& -\left(\frac{388}{3}+900 x\right) \mathrm{H}_{-2,0,0}-(140+196 x) \mathrm{H}_{4}+\left(\frac{1061}{6}-\frac{161}{6} x\right) \mathrm{H}_{0} \zeta_{4}+\left(\frac{544}{3}+\frac{416}{3} x\right) \mathrm{H}_{-2,2,0} \\
& -\left(216+\frac{748}{3} x\right) \mathrm{H}_{0} \zeta_{2}+\left(\frac{704}{3}+\frac{448}{3} x\right) \mathrm{H}_{-3,2}-\left(\frac{800}{3}+\frac{928}{3} x\right) \mathrm{H}_{5}+\left(279-\frac{661}{3} x\right) \mathrm{H}_{0} \zeta_{3} \\
& -(304-112 x) \mathrm{H}_{-2,-1,0,0}-\left(\frac{988}{3}+\frac{548}{3} x\right) \mathrm{H}_{-2,0} \zeta_{2}-\left(\frac{1016}{3}+\frac{136}{3} x\right) \mathrm{H}_{-3} \zeta_{2} \\
& +\left(\frac{1184}{3}+\frac{928}{3} x\right) \mathrm{H}_{-2,3}+(404-260 x) \mathrm{H}_{-4,0}+(408+72 x) \mathrm{H}_{0,0} \zeta_{3}+(420+60 x) \mathrm{H}_{0,0,0} \zeta_{2} \\
& +\left(\frac{1541}{3}+\frac{1565}{3} x\right) \mathrm{H}_{0,0}+(524+826 x) \mathrm{H}_{0,0,0}-(600-40 x) \mathrm{H}_{0,0,0,0,0}+(604-124 x) \mathrm{H}_{-2,0,0,0} \\
& +(608-320 x) \mathrm{H}_{-3,0,0}+p_{\mathrm{qq}}(-x)\left(-408 \mathrm{H}_{-4} \zeta_{2}-416 \mathrm{H}_{-3} \zeta_{3}+192 \mathrm{H}_{-3} \zeta_{2}-150 \mathrm{H}_{-2} \zeta_{4}\right. \\
& -\frac{304}{3} \mathrm{H}_{-2} \zeta_{3}+\frac{2300}{3} \mathrm{H}_{-2} \zeta_{2}+416 \mathrm{H}_{0} \zeta_{5}-\frac{335}{6} \mathrm{H}_{0} \zeta_{4}-\frac{1993}{3} \mathrm{H}_{0} \zeta_{3}+\frac{157}{2} \mathrm{H}_{0} \zeta_{2}-164 \mathrm{H}_{0} \zeta_{2} \zeta_{3} \\
& +24 \mathrm{H}_{3}-48 \mathrm{H}_{3} \zeta_{3}-24 \mathrm{H}_{3} \zeta_{2}+614 \mathrm{H}_{4}-72 \mathrm{H}_{4} \zeta_{2}+240 \mathrm{H}_{5}-320 \mathrm{H}_{6}+192 \mathrm{H}_{-5,0}-460 \mathrm{H}_{-4,0} \\
& +336 \mathrm{H}_{-4,2}+384 \mathrm{H}_{-3,-1} \zeta_{2}-\frac{1880}{3} \mathrm{H}_{-3,0}-456 \mathrm{H}_{-3,0} \zeta_{2}-\frac{224}{3} \mathrm{H}_{-3,2}+544 \mathrm{H}_{-3,3} \\
& +384 \mathrm{H}_{-2,-2} \zeta_{2}+416 \mathrm{H}_{-2,-1} \zeta_{3}+\frac{128}{3} \mathrm{H}_{-2,-1} \zeta_{2}+181 \mathrm{H}_{-2,0}-448 \mathrm{H}_{-2,0} \zeta_{3}+\frac{616}{3} \mathrm{H}_{-2,0} \zeta_{2} \\
& -\frac{1228}{3} \mathrm{H}_{-2,2}+16 \mathrm{H}_{-2,2} \zeta_{2}-\frac{304}{3} \mathrm{H}_{-2,3}+624 \mathrm{H}_{-2,4}+384 \mathrm{H}_{-1,-3} \zeta_{2}+416 \mathrm{H}_{-1,-2} \zeta_{3} \\
& +\frac{128}{3} \mathrm{H}_{-1,-2} \zeta_{2}-150 \mathrm{H}_{-1,0} \zeta_{4}-\frac{304}{3} \mathrm{H}_{-1,0} \zeta_{3}+\frac{2300}{3} \mathrm{H}_{-1,0} \zeta_{2}-\frac{2456}{3} \mathrm{H}_{-1,3}+32 \mathrm{H}_{-1,3} \zeta_{2} \\
& -80 \mathrm{H}_{-1,4}+576 \mathrm{H}_{-1,5}-18 \mathrm{H}_{0,0}+316 \mathrm{H}_{0,0} \zeta_{4}-\frac{632}{3} \mathrm{H}_{0,0} \zeta_{3}-\frac{2060}{3} \mathrm{H}_{0,0} \zeta_{2}+12 \mathrm{H}_{2,0}-24 \mathrm{H}_{2,0} \zeta_{3} \\
& -12 \mathrm{H}_{2,0} \zeta_{2}-32 \mathrm{H}_{2,2} \zeta_{2}+\frac{614}{3} \mathrm{H}_{3,0}-56 \mathrm{H}_{3,0} \zeta_{2}-64 \mathrm{H}_{3,1} \zeta_{2}-\frac{208}{3} \mathrm{H}_{3,2}+44 \mathrm{H}_{4,0}-208 \mathrm{H}_{4,1} \\
& -32 \mathrm{H}_{4,2}-128 \mathrm{H}_{5,0}-128 \mathrm{H}_{5,1}-144 \mathrm{H}_{-4,-1,0}+336 \mathrm{H}_{-4,0,0}-48 \mathrm{H}_{-3,-2,0}+\frac{704}{3} \mathrm{H}_{-3,-1,0}
\end{aligned}
$$




$$
\begin{aligned}
& -384 \mathrm{H}_{-3,-1,2}-696 \mathrm{H}_{-3,0,0}+240 \mathrm{H}_{-3,2,0}+256 \mathrm{H}_{-3,2,1}-48 \mathrm{H}_{-2,-3,0}+\frac{704}{3} \mathrm{H}_{-2,-2,0} \\
& -384 \mathrm{H}_{-2,-2,2}-384 \mathrm{H}_{-2,-1,-1} \zeta_{2}+\frac{2144}{3} \mathrm{H}_{-2,-1,0}+512 \mathrm{H}_{-2,-1,0} \zeta_{2}-\frac{128}{3} \mathrm{H}_{-2,-1,2} \\
& -704 \mathrm{H}_{-2,-1,3}-\frac{3302}{3} \mathrm{H}_{-2,0,0}-480 \mathrm{H}_{-2,0,0} \zeta_{2}-\frac{80}{3} \mathrm{H}_{-2,2,0}+\frac{416}{3} \mathrm{H}_{-2,2,1}+128 \mathrm{H}_{-2,2,2} \\
& +352 \mathrm{H}_{-2,3,0}+384 \mathrm{H}_{-2,3,1}-144 \mathrm{H}_{-1,-4,0}+\frac{704}{3} \mathrm{H}_{-1,-3,0}-384 \mathrm{H}_{-1,-3,2}-384 \mathrm{H}_{-1,-2,-1} \zeta_{2} \\
& +\frac{2144}{3} \mathrm{H}_{-1,-2,0}+512 \mathrm{H}_{-1,-2,0} \zeta_{2}-\frac{128}{3} \mathrm{H}_{-1,-2,2}-704 \mathrm{H}_{-1,-2,3}-384 \mathrm{H}_{-1,-1,-2} \zeta_{2} \\
& +416 \mathrm{H}_{-1,-1,0} \zeta_{3}+\frac{128}{3} \mathrm{H}_{-1,-1,0} \zeta_{2}-\frac{256}{3} \mathrm{H}_{-1,-1,3}-960 \mathrm{H}_{-1,-1,4}+362 \mathrm{H}_{-1,0,0}-480 \mathrm{H}_{-1,0,0} \zeta_{3} \\
& +\frac{656}{3} \mathrm{H}_{-1,0,0} \zeta_{2}-\frac{1228}{3} \mathrm{H}_{-1,2,0}+16 \mathrm{H}_{-1,2,0} \zeta_{2}+\frac{416}{3} \mathrm{H}_{-1,2,2}-\frac{16}{3} \mathrm{H}_{-1,3,0}+\frac{832}{3} \mathrm{H}_{-1,3,1} \\
& +128 \mathrm{H}_{-1,3,2}+336 \mathrm{H}_{-1,4,0}+384 \mathrm{H}_{-1,4,1}-\frac{507}{2} \mathrm{H}_{0,0,0}+384 \mathrm{H}_{0,0,0} \zeta_{3}-464 \mathrm{H}_{0,0,0} \zeta_{2} \\
& -32 \mathrm{H}_{2,0,0} \zeta_{2}-32 \mathrm{H}_{2,1,0} \zeta_{2}-\frac{32}{3} \mathrm{H}_{3,0,0}-\frac{208}{3} \mathrm{H}_{3,1,0}-32 \mathrm{H}_{4,0,0}-32 \mathrm{H}_{4,1,0}-288 \mathrm{H}_{-3,-1,0,0} \\
& +432 \mathrm{H}_{-3,0,0,0}-192 \mathrm{H}_{-2,-2,0,0}+384 \mathrm{H}_{-2,-1,-1,2}+448 \mathrm{H}_{-2,-1,0,0}-320 \mathrm{H}_{-2,-1,2,0} \\
& -256 \mathrm{H}_{-2,-1,2,1}-856 \mathrm{H}_{-2,0,0,0}+128 \mathrm{H}_{-2,2,0,0}+128 \mathrm{H}_{-2,2,1,0}-288 \mathrm{H}_{-1,-3,0,0} \\
& +384 \mathrm{H}_{-1,-2,-1,2}+448 \mathrm{H}_{-1,-2,0,0}-320 \mathrm{H}_{-1,-2,2,0}-256 \mathrm{H}_{-1,-2,2,1}+384 \mathrm{H}_{-1,-1,-2,2} \\
& -384 \mathrm{H}_{-1,-1,-1,0} \zeta_{2}+768 \mathrm{H}_{-1,-1,-1,3}+\frac{4288}{3} \mathrm{H}_{-1,-1,0,0}+640 \mathrm{H}_{-1,-1,0,0} \zeta_{2}-\frac{128}{3} \mathrm{H}_{-1,-1,2,0} \\
& -256 \mathrm{H}_{-1,-1,2,2}-576 \mathrm{H}_{-1,-1,3,0}-512 \mathrm{H}_{-1,-1,3,1}-1422 \mathrm{H}_{-1,0,0,0}-480 \mathrm{H}_{-1,0,0,0} \zeta_{2} \\
& +\frac{64}{3} \mathrm{H}_{-1,2,0,0}+\frac{416}{3} \mathrm{H}_{-1,2,1,0}+128 \mathrm{H}_{-1,3,0,0}+128 \mathrm{H}_{-1,3,1,0}+\frac{1400}{3} \mathrm{H}_{0,0,0,0}+320 \mathrm{H}_{0,0,0,0} \zeta_{2} \\
& +192 \mathrm{H}_{-2,-1,-1,0,0}-480 \mathrm{H}_{-2,-1,0,0,0}+496 \mathrm{H}_{-2,0,0,0,0}+192 \mathrm{H}_{-1,-2,-1,0,0}-480 \mathrm{H}_{-1,-2,0,0,0} \\
& +192 \mathrm{H}_{-1,-1,-2,0,0}+384 \mathrm{H}_{-1,-1,-1,2,0}+640 \mathrm{H}_{-1,-1,0,0,0}-256 \mathrm{H}_{-1,-1,2,0,0}-256 \mathrm{H}_{-1,-1,2,1,0} \\
& -1088 \mathrm{H}_{-1,0,0,0,0}+740 \mathrm{H}_{0,0,0,0,0}+576 \mathrm{H}_{-1,-1,-1,0,0,0}-768 \mathrm{H}_{-1,-1,0,0,0,0}+560 \mathrm{H}_{-1,0,0,0,0,0} \\
& \left.\left.-288 \mathrm{H}_{0,0,0,0,0,0}\right)\right\} \\
& +\frac{16}{3} C_{F}^{2}\left(C_{A}-2 C_{F}\right)^{2}\left\{( 1 + x ) \left(-1248 \mathrm{H}_{-2} \zeta_{3}-316 \mathrm{H}_{-2} \zeta_{2}+1152 \mathrm{H}_{-2,-1} \zeta_{2}-834 \mathrm{H}_{-2,0}\right.\right. \\
& +552 \mathrm{H}_{-2,2}+1152 \mathrm{H}_{-1,-2} \zeta_{2}-1248 \mathrm{H}_{-1,0} \zeta_{3}-316 \mathrm{H}_{-1,0} \zeta_{2}+1104 \mathrm{H}_{-1,3}+2880 \mathrm{H}_{-1,4} \\
& -560 \mathrm{H}_{2,2}-1120 \mathrm{H}_{3,1}+472 \mathrm{H}_{-2,-1,0}-1152 \mathrm{H}_{-2,-1,2}+768 \mathrm{H}_{-2,2,1}+472 \mathrm{H}_{-1,-2,0} \\
& -1152 \mathrm{H}_{-1,-2,2}+1152 \mathrm{H}_{-1,-1,0} \zeta_{2}-2304 \mathrm{H}_{-1,-1,3}-1668 \mathrm{H}_{-1,0,0}-2112 \mathrm{H}_{-1,0,0} \zeta_{2} \\
& +552 \mathrm{H}_{-1,2,0}+768 \mathrm{H}_{-1,2,2}+1728 \mathrm{H}_{-1,3,0}+1536 \mathrm{H}_{-1,3,1}-560 \mathrm{H}_{2,1,0}-576 \mathrm{H}_{-1,-2,0,0} \\
& +944 \mathrm{H}_{-1,-1,0,0}-1152 \mathrm{H}_{-1,-1,2,0}-408 \mathrm{H}_{-1,0,0,0}+768 \mathrm{H}_{-1,2,0,0}+768 \mathrm{H}_{-1,2,1,0}+72 \mathrm{H}_{2,0,0,0} \\
& \left.-1728 \mathrm{H}_{-1,-1,0,0,0}+1344 \mathrm{H}_{-1,0,0,0,0}\right)+(1-x)\left(720 \mathrm{H}_{-4} \zeta_{2}+744 \mathrm{H}_{-3} \zeta_{3}+\frac{2445}{2} \mathrm{H}_{0}\right. \\
& -126 \mathrm{H}_{0} \zeta_{5}+264 \mathrm{H}_{0} \zeta_{2} \zeta_{3}-766 \mathrm{H}_{2}-72 \mathrm{H}_{2} \zeta_{3}-156 \mathrm{H}_{2} \zeta_{2}-192 \mathrm{H}_{3} \zeta_{2}+144 \mathrm{H}_{4} \zeta_{2}+300 \mathrm{H}_{6} \\
& -48 \mathrm{H}_{-5,0}+390 \mathrm{H}_{-4,0}-648 \mathrm{H}_{-4,2}-960 \mathrm{H}_{-3,-1} \zeta_{2}+816 \mathrm{H}_{-3,0} \zeta_{2}-864 \mathrm{H}_{-3,3}-480 \mathrm{H}_{-2,-2} \zeta_{2} \\
& +372 \mathrm{H}_{-2,0} \zeta_{3}-648 \mathrm{H}_{-2,4}-33 \mathrm{H}_{0,0} \zeta_{4}-766 \mathrm{H}_{1,0}-72 \mathrm{H}_{1,0} \zeta_{3}-156 \mathrm{H}_{1,0} \zeta_{2}-1120 \mathrm{H}_{1,2}
\end{aligned}
$$


$-192 \mathrm{H}_{1,2} \zeta_{2}-192 \mathrm{H}_{2,0} \zeta_{2}-1120 \mathrm{H}_{2,1}-192 \mathrm{H}_{2,1} \zeta_{2}+48 \mathrm{H}_{3,0} \zeta_{2}+60 \mathrm{H}_{5,0}+144 \mathrm{H}_{-4,-1,0}$ $-420 \mathrm{H}_{-4,0,0}+144 \mathrm{H}_{-3,-2,0}-168 \mathrm{H}_{-3,-1,0}+864 \mathrm{H}_{-3,-1,2}-216 \mathrm{H}_{-3,2,0}+96 \mathrm{H}_{-2,-3,0}$ $-84 \mathrm{H}_{-2,-2,0}+432 \mathrm{H}_{-2,-2,2}-480 \mathrm{H}_{-2,-1,0} \zeta_{2}+864 \mathrm{H}_{-2,-1,3}+576 \mathrm{H}_{-2,0,0} \zeta_{2}-216 \mathrm{H}_{-2,3,0}$ $-252 \mathrm{H}_{0,0,0} \zeta_{3}-944 \mathrm{H}_{1,0,0}-192 \mathrm{H}_{1,0,0} \zeta_{2}-1120 \mathrm{H}_{1,1,0}-192 \mathrm{H}_{1,1,0} \zeta_{2}-192 \mathrm{H}_{-3,-1,-1,0}$ $+624 \mathrm{H}_{-3,-1,0,0}-588 \mathrm{H}_{-3,0,0,0}-96 \mathrm{H}_{-2,-2,-1,0}+360 \mathrm{H}_{-2,-2,0,0}-96 \mathrm{H}_{-2,-1,-2,0}$ $+432 \mathrm{H}_{-2,-1,2,0}-264 \mathrm{H}_{0,0,0,0} \zeta_{2}+144 \mathrm{H}_{1,0,0,0}-192 \mathrm{H}_{-2,-1,-1,0,0}+792 \mathrm{H}_{-2,-1,0,0,0}$ $\left.-384 \mathrm{H}_{-2,0,0,0,0}+108 \mathrm{H}_{0,0,0,0,0,0}\right)-(144+528 x) \mathrm{H}_{3,0,0}+(162+1158 x) \mathrm{H}_{0,0} \zeta_{2}$ $-(174+956 x) \mathrm{H}_{0} \zeta_{2}-(192+576 x)\left(\mathrm{H}_{3,2}+\mathrm{H}_{3,1,0}\right)-(196+1100 x) \mathrm{H}_{-3,0}$ $-\left(\frac{799}{4}+\frac{3761}{4} x\right) \mathrm{H}_{0} \zeta_{4}+(324+428 x) \mathrm{H}_{0,0,0,0}-(332+1236 x) \mathrm{H}_{-2,0,0}-(424+520 x) \mathrm{H}_{2,0,0}$ $-(424+1352 x) \mathrm{H}_{4,0}+(447+469 x) \mathrm{H}_{0} \zeta_{3}-(544+1952 x) \mathrm{H}_{5}-(552+2256 x) \mathrm{H}_{4}$ $+(576+1152 x) \mathrm{H}_{0,0} \zeta_{3}-(576+1728 x) \mathrm{H}_{4,1}-(645+75 x) \mathrm{H}_{0,0,0,0,0}-(648-1240 x) \mathrm{H}_{3}$ $-(656+1224 x) \mathrm{H}_{3,0}+(657+1503 x) \mathrm{H}_{0,0,0} \zeta_{2}-(744+408 x) \mathrm{H}_{-2,-1,0,0}-(796-1092 x) \mathrm{H}_{2,0}$ $+(800+1120 x) \mathrm{H}_{-2,2,0}+(832+1472 x) \mathrm{H}_{-3,2}-(916+1388 x) \mathrm{H}_{-3} \zeta_{2}+(936+216 x) \mathrm{H}_{-3,0,0}$ $+(1209+1869 x) \mathrm{H}_{0,0,0}+(1350+1050 x) \mathrm{H}_{-2,0,0,0}+(1486+2578 x) \mathrm{H}_{0,0}$ $-(1514+1750 x) \mathrm{H}_{-2,0} \zeta_{2}+(1792+2432 x) \mathrm{H}_{-2,3}+p_{\mathrm{qq}}(-x)\left(-1296 \mathrm{H}_{-4} \zeta_{2}-2496 \mathrm{H}_{-3} \zeta_{3}\right.$ $+576 \mathrm{H}_{-3} \zeta_{2}+1140 \mathrm{H}_{-2} \zeta_{4}-8 \mathrm{H}_{-2} \zeta_{3}+1204 \mathrm{H}_{-2} \zeta_{2}+258 \mathrm{H}_{0} \zeta_{5}-50 \mathrm{H}_{0} \zeta_{4}-1037 \mathrm{H}_{0} \zeta_{3}$ $+\frac{177}{2} \mathrm{H}_{0} \zeta_{2}-708 \mathrm{H}_{0} \zeta_{2} \zeta_{3}+72 \mathrm{H}_{3}-144 \mathrm{H}_{3} \zeta_{3}+72 \mathrm{H}_{3} \zeta_{2}+1002 \mathrm{H}_{4}-288 \mathrm{H}_{4} \zeta_{2}+432 \mathrm{H}_{5}$ $-720 \mathrm{H}_{6}-636 \mathrm{H}_{-4,0}+1296 \mathrm{H}_{-4,2}+2304 \mathrm{H}_{-3,-1} \zeta_{2}-940 \mathrm{H}_{-3,0}-2544 \mathrm{H}_{-3,0} \zeta_{2}-400 \mathrm{H}_{-3,2}$ $+2784 \mathrm{H}_{-3,3}+2304 \mathrm{H}_{-2,-2} \zeta_{2}+2496 \mathrm{H}_{-2,-1} \zeta_{3}-224 \mathrm{H}_{-2,-1} \zeta_{2}+249 \mathrm{H}_{-2,0}-2112 \mathrm{H}_{-2,0} \zeta_{3}$ $+632 \mathrm{H}_{-2,0} \zeta_{2}-668 \mathrm{H}_{-2,2}-512 \mathrm{H}_{-2,3}+3504 \mathrm{H}_{-2,4}+2304 \mathrm{H}_{-1,-3} \zeta_{2}+2496 \mathrm{H}_{-1,-2} \zeta_{3}$ $-224 \mathrm{H}_{-1,-2} \zeta_{2}+1140 \mathrm{H}_{-1,0} \zeta_{4}-8 \mathrm{H}_{-1,0} \zeta_{3}+1204 \mathrm{H}_{-1,0} \zeta_{2}-1336 \mathrm{H}_{-1,3}-336 \mathrm{H}_{-1,4}$ $+2496 \mathrm{H}_{-1,5}-54 \mathrm{H}_{0,0}-444 \mathrm{H}_{0,0} \zeta_{4}-316 \mathrm{H}_{0,0} \zeta_{3}-1084 \mathrm{H}_{0,0} \zeta_{2}+36 \mathrm{H}_{2,0}-72 \mathrm{H}_{2,0} \zeta_{3}$ $+36 \mathrm{H}_{2,0} \zeta_{2}-192 \mathrm{H}_{2,2} \zeta_{2}+334 \mathrm{H}_{3,0}-288 \mathrm{H}_{3,0} \zeta_{2}-384 \mathrm{H}_{3,1} \zeta_{2}-176 \mathrm{H}_{3,2}-24 \mathrm{H}_{4,0}-528 \mathrm{H}_{4,1}$ $-192 \mathrm{H}_{4,2}-528 \mathrm{H}_{5,0}-768 \mathrm{H}_{5,1}+648 \mathrm{H}_{-4,0,0}+352 \mathrm{H}_{-3,-1,0}-2304 \mathrm{H}_{-3,-1,2}-1152 \mathrm{H}_{-3,0,0}$ $+1200 \mathrm{H}_{-3,2,0}+1536 \mathrm{H}_{-3,2,1}+352 \mathrm{H}_{-2,-2,0}-2304 \mathrm{H}_{-2,-2,2}-2304 \mathrm{H}_{-2,-1,-1} \zeta_{2}$ $+1072 \mathrm{H}_{-2,-1,0}+3264 \mathrm{H}_{-2,-1,0} \zeta_{2}+224 \mathrm{H}_{-2,-1,2}-4224 \mathrm{H}_{-2,-1,3}-1678 \mathrm{H}_{-2,0,0}$ $-2832 \mathrm{H}_{-2,0,0} \zeta_{2}-112 \mathrm{H}_{-2,2,0}+352 \mathrm{H}_{-2,2,1}+768 \mathrm{H}_{-2,2,2}+2112 \mathrm{H}_{-2,3,0}+2304 \mathrm{H}_{-2,3,1}$ $+352 \mathrm{H}_{-1,-3,0}-2304 \mathrm{H}_{-1,-3,2}-2304 \mathrm{H}_{-1,-2,-1} \zeta_{2}+1072 \mathrm{H}_{-1,-2,0}+3264 \mathrm{H}_{-1,-2,0} \zeta_{2}$ $+224 \mathrm{H}_{-1,-2,2}-4224 \mathrm{H}_{-1,-2,3}-2304 \mathrm{H}_{-1,-1,-2} \zeta_{2}+2496 \mathrm{H}_{-1,-1,0} \zeta_{3}-224 \mathrm{H}_{-1,-1,0} \zeta_{2}$ $+448 \mathrm{H}_{-1,-1,3}-5760 \mathrm{H}_{-1,-1,4}+498 \mathrm{H}_{-1,0,0}-1728 \mathrm{H}_{-1,0,0} \zeta_{3}+688 \mathrm{H}_{-1,0,0} \zeta_{2}-668 \mathrm{H}_{-1,2,0}$ $+352 \mathrm{H}_{-1,2,2}+64 \mathrm{H}_{-1,3,0}+704 \mathrm{H}_{-1,3,1}+768 \mathrm{H}_{-1,3,2}+1776 \mathrm{H}_{-1,4,0}+2304 \mathrm{H}_{-1,4,1}$ $-\frac{639}{2} \mathrm{H}_{0,0,0}+576 \mathrm{H}_{0,0,0} \zeta_{3}-804 \mathrm{H}_{0,0,0} \zeta_{2}-192 \mathrm{H}_{2,0,0} \zeta_{2}-192 \mathrm{H}_{2,1,0} \zeta_{2}-88 \mathrm{H}_{3,0,0}-176 \mathrm{H}_{3,1,0}$ $-120 \mathrm{H}_{4,0,0}-192 \mathrm{H}_{4,1,0}-1152 \mathrm{H}_{-3,-1,0,0}+1320 \mathrm{H}_{-3,0,0,0}-1152 \mathrm{H}_{-2,-2,0,0}+2304 \mathrm{H}_{-2,-1,-1,2}$ 


$$
\begin{aligned}
& +816 \mathrm{H}_{-2,-1,0,0}-1920 \mathrm{H}_{-2,-1,2,0}-1536 \mathrm{H}_{-2,-1,2,1}-1500 \mathrm{H}_{-2,0,0,0}+720 \mathrm{H}_{-2,2,0,0} \\
& +768 \mathrm{H}_{-2,2,1,0}-1152 \mathrm{H}_{-1,-3,0,0}+2304 \mathrm{H}_{-1,-2,-1,2}+816 \mathrm{H}_{-1,-2,0,0}-1920 \mathrm{H}_{-1,-2,2,0} \\
& -1536 \mathrm{H}_{-1,-2,2,1}+2304 \mathrm{H}_{-1,-1,-2,2}-2304 \mathrm{H}_{-1,-1,-1,0} \zeta_{2}+4608 \mathrm{H}_{-1,-1,-1,3} \\
& +2144 \mathrm{H}_{-1,-1,0,0}+4224 \mathrm{H}_{-1,-1,0,0} \zeta_{2}+224 \mathrm{H}_{-1,-1,2,0}-1536 \mathrm{H}_{-1,-1,2,2}-3456 \mathrm{H}_{-1,-1,3,0} \\
& -3072 \mathrm{H}_{-1,-1,3,1}-2214 \mathrm{H}_{-1,0,0,0}-2160 \mathrm{H}_{-1,0,0,0} \zeta_{2}+176 \mathrm{H}_{-1,2,0,0}+352 \mathrm{H}_{-1,2,1,0} \\
& +672 \mathrm{H}_{-1,3,0,0}+768 \mathrm{H}_{-1,3,1,0}+700 \mathrm{H}_{0,0,0,0}+576 \mathrm{H}_{0,0,0,0} \zeta_{2}+72 \mathrm{H}_{3,0,0,0}+1152 \mathrm{H}_{-2,-1,-1,0,0} \\
& -2400 \mathrm{H}_{-2,-1,0,0,0}+1488 \mathrm{H}_{-2,0,0,0,0}+1152 \mathrm{H}_{-1,-2,-1,0,0}-2400 \mathrm{H}_{-1,-2,0,0,0} \\
& +1152 \mathrm{H}_{-1,-1,-2,0,0}+2304 \mathrm{H}_{-1,-1,-1,2,0}+1392 \mathrm{H}_{-1,-1,0,0,0}-1536 \mathrm{H}_{-1,-1,2,0,0} \\
& -1536 \mathrm{H}_{-1,-1,2,1,0}-1632 \mathrm{H}_{-1,0,0,0,0}-144 \mathrm{H}_{-1,2,0,0,0}+1020 \mathrm{H}_{0,0,0,0,0}+3456 \mathrm{H}_{-1,-1,-1,0,0,0} \\
& \left.\left.-2688 \mathrm{H}_{-1,-1,0,0,0,0}+720 \mathrm{H}_{-1,0,0,0,0,0}-144 \mathrm{H}_{0,0,0,0,0,0}\right)\right\} \\
& +\frac{16}{3} C_{F}^{2}\left(C_{A}-2 C_{F}\right) n_{f}\left\{( 1 + x ) \left(-32 \mathrm{H}_{-2} \zeta_{2}+120 \mathrm{H}_{-2,0}-32 \mathrm{H}_{-1,0} \zeta_{2}+32 \mathrm{H}_{2,2}+64 \mathrm{H}_{3,1}\right.\right. \\
& -64 \mathrm{H}_{-2,-1,0}-64 \mathrm{H}_{-1,-2,0}+240 \mathrm{H}_{-1,0,0}+16 \mathrm{H}_{2,0,0}+32 \mathrm{H}_{2,1,0}-128 \mathrm{H}_{-1,-1,0,0} \\
& \left.+192 \mathrm{H}_{-1,0,0,0}\right)+(1-x)\left(-32 \mathrm{H}_{-3} \zeta_{2}-201 \mathrm{H}_{0}+4 \mathrm{H}_{0} \zeta_{4}-68 \mathrm{H}_{2}-24 \mathrm{H}_{4}-32 \mathrm{H}_{5}-48 \mathrm{H}_{-4,0}\right. \\
& +32 \mathrm{H}_{-3,2}-16 \mathrm{H}_{-2,0} \zeta_{2}+32 \mathrm{H}_{-2,3}-68 \mathrm{H}_{1,0}+64 \mathrm{H}_{1,2}+64 \mathrm{H}_{2,1}-8 \mathrm{H}_{4,0}-48 \mathrm{H}_{-3,0,0} \\
& \left.+16 \mathrm{H}_{-2,2,0}+32 \mathrm{H}_{1,0,0}+64 \mathrm{H}_{1,1,0}-24 \mathrm{H}_{-2,0,0,0}+60 \mathrm{H}_{0,0,0,0,0}\right)+(8+24 x) \mathrm{H}_{3,0} \\
& +(16+176 x) \mathrm{H}_{-3,0}+(24+72 x) \mathrm{H}_{0,0} \zeta_{2}-(32+96 x) \mathrm{H}_{2,0}-(36-68 x) \mathrm{H}_{0} \zeta_{3} \\
& -(48+272 x) \mathrm{H}_{0,0,0,0}+(80+240 x) \mathrm{H}_{-2,0,0}-(96+160 x) \mathrm{H}_{3}+(108+140 x) \mathrm{H}_{0} \zeta_{2} \\
& -(228+348 x) \mathrm{H}_{0,0,0}-(244+28 x) \mathrm{H}_{0,0}+p_{\mathrm{qq}}(-x)\left(80 \mathrm{H}_{-2} \zeta_{3}-184 \mathrm{H}_{-2} \zeta_{2}+5 \mathrm{H}_{0} \zeta_{4}\right. \\
& +158 \mathrm{H}_{0} \zeta_{3}-21 \mathrm{H}_{0} \zeta_{2}-156 \mathrm{H}_{4}+96 \mathrm{H}_{-4,0}+136 \mathrm{H}_{-3,0}-32 \mathrm{H}_{-3,2}-64 \mathrm{H}_{-2,-1} \zeta_{2}-42 \mathrm{H}_{-2,0} \\
& +16 \mathrm{H}_{-2,0} \zeta_{2}+104 \mathrm{H}_{-2,2}-64 \mathrm{H}_{-2,3}-64 \mathrm{H}_{-1,-2} \zeta_{2}+80 \mathrm{H}_{-1,0} \zeta_{3}-184 \mathrm{H}_{-1,0} \zeta_{2}+208 \mathrm{H}_{-1,3} \\
& -96 \mathrm{H}_{-1,4}-8 \mathrm{H}_{0,0} \zeta_{3}+172 \mathrm{H}_{0,0} \zeta_{2}-52 \mathrm{H}_{3,0}+32 \mathrm{H}_{3,2}+24 \mathrm{H}_{4,0}+96 \mathrm{H}_{4,1}-64 \mathrm{H}_{-3,-1,0} \\
& +144 \mathrm{H}_{-3,0,0}-64 \mathrm{H}_{-2,-2,0}-160 \mathrm{H}_{-2,-1,0}+64 \mathrm{H}_{-2,-1,2}+244 \mathrm{H}_{-2,0,0}-32 \mathrm{H}_{-2,2,0}-64 \mathrm{H}_{-2,2,1} \\
& -64 \mathrm{H}_{-1,-3,0}-160 \mathrm{H}_{-1,-2,0}+64 \mathrm{H}_{-1,-2,2}-64 \mathrm{H}_{-1,-1,0} \zeta_{2}+128 \mathrm{H}_{-1,-1,3}-84 \mathrm{H}_{-1,0,0} \\
& +32 \mathrm{H}_{-1,0,0} \zeta_{2}+104 \mathrm{H}_{-1,2,0}-64 \mathrm{H}_{-1,2,2}-64 \mathrm{H}_{-1,3,0}-128 \mathrm{H}_{-1,3,1}+63 \mathrm{H}_{0,0,0}+48 \mathrm{H}_{0,0,0} \zeta_{2} \\
& +16 \mathrm{H}_{3,0,0}+32 \mathrm{H}_{3,1,0}-96 \mathrm{H}_{-2,-1,0,0}+168 \mathrm{H}_{-2,0,0,0}-96 \mathrm{H}_{-1,-2,0,0}-320 \mathrm{H}_{-1,-1,0,0} \\
& +64 \mathrm{H}_{-1,-1,2,0}+324 \mathrm{H}_{-1,0,0,0}-32 \mathrm{H}_{-1,2,0,0}-64 \mathrm{H}_{-1,2,1,0}-112 \mathrm{H}_{0,0,0,0}-96 \mathrm{H}_{-1,-1,0,0,0} \\
& \left.\left.+192 \mathrm{H}_{-1,0,0,0,0}-120 \mathrm{H}_{0,0,0,0,0}\right)\right\} \text {. }
\end{aligned}
$$

A difference between the time-like and space-like case appears for the quantities $P_{\mathrm{ns}}^{(3) \mathrm{s}}$ for the first time at the four-loop level with

$$
\begin{aligned}
& \delta P^{(3) \mathrm{s}}(x)= \\
& -\frac{16}{3} n_{f} C_{F} \frac{d^{a b c} d_{a b c}}{N_{R}}\left\{( 1 + x ) \left(-336 \mathrm{H}_{-2} \zeta_{3}+648 \mathrm{H}_{-2} \zeta_{2}+384 \mathrm{H}_{3} \zeta_{3}-576 \mathrm{H}_{4} \zeta_{2}-328 \mathrm{H}_{-2,0}\right.\right.
\end{aligned}
$$


$-256 \mathrm{H}_{-2,2}-336 \mathrm{H}_{-1,0} \zeta_{3}+648 \mathrm{H}_{-1,0} \zeta_{2}-512 \mathrm{H}_{-1,3}+1440 \mathrm{H}_{-1,4}+192 \mathrm{H}_{2,0} \zeta_{3}+1312 \mathrm{H}_{2,2}$ $-384 \mathrm{H}_{2,2} \zeta_{2}-288 \mathrm{H}_{2,4}-384 \mathrm{H}_{3,0} \zeta_{2}+2624 \mathrm{H}_{3,1}-768 \mathrm{H}_{3,1} \zeta_{2}-192 \mathrm{H}_{3,3}+576 \mathrm{H}_{4,2}+2304 \mathrm{H}_{5,1}$ $+784 \mathrm{H}_{-2,-1,0}-192 \mathrm{H}_{-2,-1,2}+768 \mathrm{H}_{-2,2,1}+384 \mathrm{H}_{-1,-3,0}+784 \mathrm{H}_{-1,-2,0}-192 \mathrm{H}_{-1,-2,2}$ $-384 \mathrm{H}_{-1,-1,3}-656 \mathrm{H}_{-1,0,0}-768 \mathrm{H}_{-1,0,0} \zeta_{2}-256 \mathrm{H}_{-1,2,0}+768 \mathrm{H}_{-1,2,2}+1248 \mathrm{H}_{-1,3,0}$ $+1536 \mathrm{H}_{-1,3,1}-192 \mathrm{H}_{2,0,0} \zeta_{2}+1312 \mathrm{H}_{2,1,0}-384 \mathrm{H}_{2,1,0} \zeta_{2}-96 \mathrm{H}_{2,3,0}+432 \mathrm{H}_{4,0,0}+576 \mathrm{H}_{4,1,0}$ $-384 \mathrm{H}_{-2,-1,-1,0}-384 \mathrm{H}_{-1,-2,-1,0}+480 \mathrm{H}_{-1,-2,0,0}-384 \mathrm{H}_{-1,-1,-2,0}+1568 \mathrm{H}_{-1,-1,0,0}$ $-192 \mathrm{H}_{-1,-1,2,0}-1560 \mathrm{H}_{-1,0,0,0}+768 \mathrm{H}_{-1,2,0,0}+768 \mathrm{H}_{-1,2,1,0}-96 \mathrm{H}_{2,2,0,0}-336 \mathrm{H}_{3,0,0,0}$ $-192 \mathrm{H}_{3,1,0,0}-768 \mathrm{H}_{-1,-1,-1,0,0}+288 \mathrm{H}_{-1,-1,0,0,0}+384 \mathrm{H}_{-1,0,0,0,0}-384 \mathrm{H}_{2,0,0,0,0}$ $\left.-288 \mathrm{H}_{2,1,0,0,0}\right)+(1-x)\left(1152 \mathrm{H}_{-4} \zeta_{2}+1344 \mathrm{H}_{-3} \zeta_{3}+3232 \mathrm{H}_{0}+4684 \mathrm{H}_{2}+288 \mathrm{H}_{2} \zeta_{3}\right.$ $-392 \mathrm{H}_{2} \zeta_{2}-384 \mathrm{H}_{-5,0}-576 \mathrm{H}_{-4,2}-1536 \mathrm{H}_{-3,-1} \zeta_{2}+1152 \mathrm{H}_{-3,0} \zeta_{2}-768 \mathrm{H}_{-3,3}$ $-768 \mathrm{H}_{-2,-2} \zeta_{2}+672 \mathrm{H}_{-2,0} \zeta_{3}-576 \mathrm{H}_{-2,4}+4684 \mathrm{H}_{1,0}+288 \mathrm{H}_{1,0} \zeta_{3}-392 \mathrm{H}_{1,0} \zeta_{2}+2912 \mathrm{H}_{1,2}$ $-432 \mathrm{H}_{1,4}+2912 \mathrm{H}_{2,1}-144 \mathrm{H}_{2,3}+1152 \mathrm{H}_{-4,-1,0}-1056 \mathrm{H}_{-4,0,0}+1152 \mathrm{H}_{-3,-2,0}$ $+768 \mathrm{H}_{-3,-1,2}-192 \mathrm{H}_{-3,2,0}+768 \mathrm{H}_{-2,-3,0}+384 \mathrm{H}_{-2,-2,2}-768 \mathrm{H}_{-2,-1,0} \zeta_{2}+768 \mathrm{H}_{-2,-1,3}$ $+768 \mathrm{H}_{-2,0,0} \zeta_{2}-192 \mathrm{H}_{-2,3,0}+2912 \mathrm{H}_{1,0,0}+2912 \mathrm{H}_{1,1,0}-144 \mathrm{H}_{1,3,0}-1536 \mathrm{H}_{-3,-1,-1,0}$ $+1920 \mathrm{H}_{-3,-1,0,0}-1248 \mathrm{H}_{-3,0,0,0}-768 \mathrm{H}_{-2,-2,-1,0}+1344 \mathrm{H}_{-2,-2,0,0}-768 \mathrm{H}_{-2,-1,-2,0}$ $+384 \mathrm{H}_{-2,-1,2,0}-396 \mathrm{H}_{1,0,0,0}-144 \mathrm{H}_{1,2,0,0}-144 \mathrm{H}_{2,1,0,0}-1536 \mathrm{H}_{-2,-1,-1,0,0}$ $\left.+1728 \mathrm{H}_{-2,-1,0,0,0}-768 \mathrm{H}_{-2,0,0,0,0}-576 \mathrm{H}_{1,0,0,0,0}-432 \mathrm{H}_{1,1,0,0,0}\right)$ $-\left(96-1056 x+1024 x^{2}\right) \mathrm{H}_{-2,-1,0,0}+\left(96-864 x-512 x^{2}\right)\left(\mathrm{H}_{3,2}+\mathrm{H}_{3,1,0}\right)$ $+\left(96-96 x+512 x^{2}\right) \mathrm{H}_{1,0,0} \zeta_{2}+\left(96+192 x+512 x^{2}\right) \mathrm{H}_{2,0} \zeta_{2}+\left(96+480 x+512 x^{2}\right) \mathrm{H}_{3} \zeta_{2}$ $+\left(96+672 x-512 x^{2}\right) \mathrm{H}_{-2,-2,0}-(144-240 x) \mathrm{H}_{0} \zeta_{5}-\left(192-960 x+1024 x^{2}\right) \mathrm{H}_{-3,-1,0}$ $-\left(192-192 x-512 x^{2}\right)\left(\mathrm{H}_{1,2} \zeta_{2}+\mathrm{H}_{2,1} \zeta_{2}+\mathrm{H}_{1,1,0} \zeta_{2}\right)+\left(288-2592 x-1536 x^{2}\right) \mathrm{H}_{4,1}$ $+\left(288-576 x+768 x^{2}\right) \mathrm{H}_{-4,0}-(288-432 x) \mathrm{H}_{2,0,0,0}+\left(372-1680 x-736 x^{2}\right) \mathrm{H}_{0} \zeta_{4}$ $-(400+1168 x) \mathrm{H}_{-3,0}-\left(480+1280 x^{2}\right) \mathrm{H}_{0,0,0,0,0}+\left(576-960 x+1536 x^{2}\right) \mathrm{H}_{-3,0,0}$ $+\left(576-192 x+1024 x^{2}\right) \mathrm{H}_{-3,2}-\left(576+1152 x+2048 x^{2}\right) \mathrm{H}_{5}-(976+1504 x) \mathrm{H}_{0} \zeta_{3}$ $+\left(672-768 x+1536 x^{2}\right) \mathrm{H}_{-2,0,0,0}-\left(672-672 x+1536 x^{2}\right) \mathrm{H}_{-3} \zeta_{2}+(1184+1856 x) \mathrm{H}_{0,0,0,0}$ $+\left(672+288 x+512 x^{2}\right) \mathrm{H}_{-2,2,0}-\left(720+48 x+768 x^{2}\right) \mathrm{H}_{-2,0} \zeta_{2}-(2280+1256 x) \mathrm{H}_{0,0} \zeta_{2}$ $+\left(720+864 x+2304 x^{2}\right) \mathrm{H}_{0,0,0} \zeta_{2}+\left(768+1792 x^{2}\right) \mathrm{H}_{0,0} \zeta_{3}-(920+1688 x) \mathrm{H}_{-2,0,0}$ $+(1056+288 x) \mathrm{H}_{0} \zeta_{2} \zeta_{3}+\left(1056+288 x+1024 x^{2}\right) \mathrm{H}_{-2,3}+(1180+1444 x) \mathrm{H}_{2,0,0}$ $+(1488+1296 x) \mathrm{H}_{5,0}+(1680+720 x) \mathrm{H}_{6}-(1728+576 x) \mathrm{H}_{0,0,0,0} \zeta_{2}+(2252+1940 x) \mathrm{H}_{3,0}$ $+(2280+1524 x) \mathrm{H}_{0,0,0}+(2376+2040 x) \mathrm{H}_{0,0} \zeta_{4}-(2724-516 x) \mathrm{H}_{0} \zeta_{2}+(2820+1884 x) \mathrm{H}_{4}$ $+(3232-6136 x) \mathrm{H}_{0,0}+(3852-1972 x) \mathrm{H}_{2,0}+(4792-1032 x) \mathrm{H}_{3}-\left(1584 x+1280 x^{2}\right) \mathrm{H}_{4,0}$ $-\left(672 x+512 x^{2}\right) \mathrm{H}_{3,0,0}+\left(\frac{1}{x}+x^{2}\right)\left(-896 \mathrm{H}_{-2} \zeta_{3}+768 \mathrm{H}_{-2,-1} \zeta_{2}-640 \mathrm{H}_{-2,0} \zeta_{2}+512 \mathrm{H}_{-2,3}\right.$ $+768 \mathrm{H}_{-1,-2} \zeta_{2}-896 \mathrm{H}_{-1,0} \zeta_{3}+1536 \mathrm{H}_{-1,4}-256 \mathrm{H}_{1,2} \zeta_{2}-128 \mathrm{H}_{2,0} \zeta_{2}-256 \mathrm{H}_{2,1} \zeta_{2}$ $-256 \mathrm{H}_{-2,-2,0}-512 \mathrm{H}_{-2,-1,2}+256 \mathrm{H}_{-2,2,0}+512 \mathrm{H}_{-2,2,1}-512 \mathrm{H}_{-1,-3,0}-512 \mathrm{H}_{-1,-2,2}$ 


$$
\begin{aligned}
& +768 \mathrm{H}_{-1,-1,0} \zeta_{2}-1024 \mathrm{H}_{-1,-1,3}-1280 \mathrm{H}_{-1,0,0} \zeta_{2}+512 \mathrm{H}_{-1,2,2}+1024 \mathrm{H}_{-1,3,0} \\
& +1024 \mathrm{H}_{-1,3,1}-256 \mathrm{H}_{1,0,0} \zeta_{2}-256 \mathrm{H}_{1,1,0} \zeta_{2}+512 \mathrm{H}_{-2,-1,-1,0}-512 \mathrm{H}_{-2,-1,0,0} \\
& +256 \mathrm{H}_{-2,0,0,0}+512 \mathrm{H}_{-1,-2,-1,0}-1024 \mathrm{H}_{-1,-2,0,0}+512 \mathrm{H}_{-1,-1,-2,0}-512 \mathrm{H}_{-1,-1,2,0} \\
& \left.+512 \mathrm{H}_{-1,2,0,0}+512 \mathrm{H}_{-1,2,1,0}+1024 \mathrm{H}_{-1,-1,-1,0,0}-1536 \mathrm{H}_{-1,-1,0,0,0}+1024 \mathrm{H}_{-1,0,0,0,0}\right) \\
& \left.-1152 \mathrm{H}_{0,0,0} \zeta_{3}+576 \mathrm{H}_{0,0,0,0,0,0}\right\} \text {. }
\end{aligned}
$$

\section{The complete $\zeta_{5}$ contributions}

Here we finally present the exact expressions for the part of $\gamma_{\mathrm{ns}}^{(3) \pm}(N)$ which is proportional to $\zeta_{5}$ :

$$
\begin{aligned}
\left.\gamma_{\mathrm{ns}}^{(3)+}(N)\right|_{\zeta_{5}}= & C_{F}^{4} 320 \zeta_{5}\left(\frac{111}{12}+6 \eta-9 \eta^{2}+14 S_{-2}\right) \\
& +C_{F}^{3} C_{A} 320 \zeta_{5}\left(-\frac{59}{4}-12 \eta+18 \eta^{2}-22 S_{-2}\right) \\
& +C_{F}^{2} C_{A}^{2} 80 \zeta_{5}\left(\frac{67}{4}+\frac{67}{3} \eta+\frac{58}{3} S_{1}-58 \eta^{2}+8 S_{1} \eta-8\left(S_{1}\right)^{2}+34 S_{-2}\right) \\
& +C_{F} C_{A}^{3} \frac{80}{3} \zeta_{5}\left(\frac{13}{4}+\frac{40}{3} \eta-\frac{116}{3} S_{1}+43 \eta^{2}-16 S_{1} \eta+16\left(S_{1}\right)^{2}-6 S_{-2}\right) \\
& +\frac{d_{F}^{a b c d} d_{A}^{a b c d}}{N_{R}} 320 \zeta_{5}\left(-\frac{25}{2}+\frac{25}{3} \eta+\frac{58}{3} S_{1}-23 \eta^{2}+8 S_{1} \eta-8\left(S_{1}\right)^{2}\right) \\
& +n_{f} C_{F}^{3} 160 \zeta_{5}\left(\frac{3}{2}+\eta-2 S_{1}\right)+n_{f} C_{F}^{2} C_{A} \frac{80}{3} \zeta_{5}\left(-\frac{3}{2}-\eta+2 S_{1}\right) \\
& +n_{f} C_{F} C_{A}^{2} \frac{80}{9} \zeta_{5}\left(-\frac{33}{2}-25 \eta+26 S_{1}+12 \eta^{2}\right) \\
& +n_{f} \frac{d_{F}^{a b c d} d_{F}^{a b c d}}{N_{R}} \frac{1280}{3} \zeta_{5}\left(3-5 \eta-2 S_{1}+6 \eta^{2}\right), \\
\left.\gamma_{\mathrm{ns}}^{(3)-}(N)\right|_{\zeta_{5}} & \left.\gamma_{\mathrm{ns}}^{(3)-}(N)\right|_{\zeta_{5}} \\
& +C_{F}^{4} 320 \zeta_{5}\left(-\frac{1}{6}+\frac{29}{6} \eta+7 \eta^{2}\right)+C_{F}^{3} C_{A} 320 \zeta_{5}\left(\frac{1}{3}-\frac{20}{3} \eta-11 \eta^{2}\right) \\
& +C_{F}^{2} C_{A}^{2} 80 \zeta_{5}\left(-1+4 \eta+17 \eta^{2}\right)+C_{F} C_{A}^{3} \frac{80}{3} \zeta_{5}\left(\frac{5}{6}+\frac{47}{6} \eta-3 \eta^{2}\right) \\
& +\frac{d_{F}^{a b c d} d_{A}^{a b c d}}{N_{R}} 320 \zeta_{5}\left(-\frac{1}{6}-\frac{13}{6} \eta\right) \cdot \\
&
\end{aligned}
$$

Since only sums with $w \leq 2$ can occur with $\zeta_{5}$, the corresponding functional form is so restricted that a direct determination and verification is possible with eight even and odd moments. 
Open Access. This article is distributed under the terms of the Creative Commons Attribution License (CC-BY 4.0), which permits any use, distribution and reproduction in any medium, provided the original author(s) and source are credited.

\section{References}

[1] D.J. Gross and F. Wilczek, Asymptotically free gauge theories. 1, Phys. Rev. D 8 (1973) 3633 [INSPIRE].

[2] H. Georgi and H.D. Politzer, Electroproduction scaling in an asymptotically free theory of strong interactions, Phys. Rev. D 9 (1974) 416 [INSPIRE].

[3] G. Altarelli and G. Parisi, Asymptotic freedom in parton language, Nucl. Phys. B 126 (1977) 298 [inSPIRE].

[4] K.J. Kim and K. Schilcher, Scaling violation in the infinite momentum frame, Phys. Rev. D 17 (1978) 2800 [INSPIRE].

[5] E.G. Floratos, D.A. Ross and C.T. Sachrajda, Higher order effects in asymptotically free gauge theories: the anomalous dimensions of Wilson operators, Nucl. Phys. B 129 (1977) 66 [Erratum ibid. B 139 (1978) 545] [INSPIRE].

[6] E.G. Floratos, D.A. Ross and C.T. Sachrajda, Higher order effects in asymptotically free gauge theories: 2. Flavor singlet Wilson operators and coefficient functions, Nucl. Phys. B 152 (1979) 493 [INSPIRE].

[7] A. Gonzalez-Arroyo, C. Lopez and F.J. Yndurain, Second order contributions to the structure functions in deep inelastic scattering. 1. Theoretical calculations, Nucl. Phys. B 153 (1979) 161 [INSPIRE].

[8] A. Gonzalez-Arroyo and C. Lopez, Second order contributions to the structure functions in deep inelastic scattering. 3. The singlet case, Nucl. Phys. B 166 (1980) 429 [InSPIRE].

[9] G. Curci, W. Furmanski and R. Petronzio, Evolution of parton densities beyond leading order: the nonsinglet case, Nucl. Phys. B 175 (1980) 27 [INSPIRE].

[10] W. Furmanski and R. Petronzio, Singlet parton densities beyond leading order, Phys. Lett. 97B (1980) 437 [INSPIRE].

[11] E.G. Floratos, C. Kounnas and R. Lacaze, Higher order QCD effects in inclusive annihilation and deep inelastic scattering, Nucl. Phys. B 192 (1981) 417 [INSPIRE].

[12] R. Hamberg and W.L. van Neerven, The correct renormalization of the gluon operator in a covariant gauge, Nucl. Phys. B 379 (1992) 143 [InSPIRE].

[13] R.K. Ellis and W. Vogelsang, The evolution of parton distributions beyond leading order: the singlet case, hep-ph/9602356 [INSPIRE].

[14] S. Moch, J.A.M. Vermaseren and A. Vogt, The three loop splitting functions in QCD: the nonsinglet case, Nucl. Phys. B 688 (2004) 101 [hep-ph/0403192] [INSPIRE].

[15] A. Vogt, S. Moch and J.A.M. Vermaseren, The three-loop splitting functions in QCD: the singlet case, Nucl. Phys. B 691 (2004) 129 [hep-ph/0404111] [InSPIRE].

[16] J. Ablinger, J. Blümlein, S. Klein, C. Schneider and F. Wissbrock, The $O\left(\alpha_{s}^{3}\right)$ massive operator matrix elements of $O\left(n_{f}\right)$ for the structure function $F_{2}\left(x, Q^{2}\right)$ and transversity, Nucl. Phys. B 844 (2011) 26 [arXiv:1008.3347] [INSPIRE]. 
[17] J. Ablinger et al., The 3-loop non-singlet heavy flavor contributions and anomalous dimensions for the structure function $F_{2}\left(x, Q^{2}\right)$ and transversity, Nucl. Phys. B 886 (2014) 733 [arXiv: 1406.4654] [INSPIRE].

[18] J. Ablinger, A. Behring, J. Blümlein, A. De Freitas, A. von Manteuffel and C. Schneider, The 3-loop pure singlet heavy flavor contributions to the structure function $F_{2}\left(x, Q^{2}\right)$ and the anomalous dimension, Nucl. Phys. B 890 (2014) 48 [arXiv:1409.1135] [INSPIRE].

[19] J. Ablinger, A. Behring, J. Blümlein, A. De Freitas, A. von Manteuffel and C. Schneider, The three-loop splitting functions $P_{q g}^{(2)}$ and $P_{g g}^{\left(2, N_{F}\right)}$, Nucl. Phys. B 922 (2017) 1 [arXiv: 1705.01508] [INSPIRE].

[20] A. Accardi et al., A critical appraisal and evaluation of modern PDFs, Eur. Phys. J. C 76 (2016) 471 [arXiv:1603.08906] [INSPIRE].

[21] C. Anastasiou, C. Duhr, F. Dulat, F. Herzog and B. Mistlberger, Higgs boson gluon-fusion production in QCD at three loops, Phys. Rev. Lett. 114 (2015) 212001 [arXiv:1503.06056] [INSPIRE].

[22] J.A.M. Vermaseren, A. Vogt and S. Moch, The third-order QCD corrections to deep-inelastic scattering by photon exchange, Nucl. Phys. B 724 (2005) 3 [hep-ph/0504242] [INSPIRE].

[23] S. Moch, J.A.M. Vermaseren and A. Vogt, Third-order QCD corrections to the charged-current structure function $F_{3}$, Nucl. Phys. B 813 (2009) 220 [arXiv:0812.4168] [INSPIRE].

[24] J. Davies, A. Vogt, S. Moch and J.A.M. Vermaseren, Non-singlet coefficient functions for charged-current deep-inelastic scattering to the third order in QCD, PoS(DIS2016) 059 [arXiv:1606.08907] [INSPIRE].

[25] J. Davies, S. Moch, J.A.M. Vermaseren and A. Vogt, Third-order QCD corrections to charged-current and polarized structure function in DIS, to appear.

[26] F.A. Dreyer and A. Karlberg, Vector-boson fusion Higgs production at three loops in QCD, Phys. Rev. Lett. 117 (2016) 072001 [arXiv:1606.00840] [INSPIRE].

[27] P.A. Baikov and K.G. Chetyrkin, New four loop results in QCD, Nucl. Phys. Proc. Suppl. 160 (2006) 76 [INSPIRE].

[28] V.N. Velizhanin, Four loop anomalous dimension of the second moment of the non-singlet twist-2 operator in QCD, Nucl. Phys. B 860 (2012) 288 [arXiv:1112.3954] [INSPIRE].

[29] V.N. Velizhanin, Four loop anomalous dimension of the third and fourth moments of the non-singlet twist-2 operator in QCD, arXiv:1411.1331 [INSPIRE].

[30] P.A. Baikov, K.G. Chetyrkin and J.H. Kühn, Massless propagators, R(s) and multiloop QCD, Nucl. Part. Phys. Proc. 261-262 (2015) 3 [arXiv:1501.06739] [InSPIRE].

[31] B. Ruijl, T. Ueda, J.A.M. Vermaseren, J. Davies and A. Vogt, First Forcer results on deep-inelastic scattering and related quantities, PoS(LL2016) 071 [arXiv:1605.08408] [INSPIRE].

[32] J. Davies, A. Vogt, B. Ruijl, T. Ueda and J.A.M. Vermaseren, Large- $N_{f}$ contributions to the four-loop splitting functions in QCD, Nucl. Phys. B 915 (2017) 335 [arXiv: 1610.07477] [INSPIRE]. 
[33] B. Ruijl, T. Ueda and J.A.M. Vermaseren, Forcer, a FORM program for the parametric reduction of four-loop massless propagator diagrams, arXiv:1704.06650 [INSPIRE].

[34] J.A.M. Vermaseren, New features of FORM, math-ph/0010025 [INSPIRE].

[35] J. Kuipers, T. Ueda, J.A.M. Vermaseren and J. Vollinga, FORM version 4.0, Comput. Phys. Commun. 184 (2013) 1453 [arXiv:1203.6543] [INSPIRE].

[36] M. Tentyukov and J.A.M. Vermaseren, The multithreaded version of FORM, Comput. Phys. Commun. 181 (2010) 1419 [hep-ph/0702279] [INSPIRE].

[37] J.A.M. Vermaseren, Harmonic sums, Mellin transforms and integrals, Int. J. Mod. Phys. A 14 (1999) 2037 [hep-ph/9806280] [INSPIRE].

[38] J. Blümlein and S. Kurth, Harmonic sums and Mellin transforms up to two loop order, Phys. Rev. D 60 (1999) 014018 [hep-ph/9810241] [INSPIRE].

[39] A.K. Lenstra, H.W. Lenstra and L. Lovász, Factoring polynomials with rational coefficients, Math. Ann. 261 (1982) 515.

[40] K. Matthews, Solving $a x=b$ using the Hermite normal form, unpublished.

[41] J.H. Silverman, The Xedni calculus and the elliptic curve discrete logarithm problem, Designs, Codes Crypt. 20 (2000) 5.

[42] CALC webpage, http://www.numbertheory.org/calc/krm_calc.html.

[43] V.N. Velizhanin, Three loop anomalous dimension of the non-singlet transversity operator in QCD, Nucl. Phys. B 864 (2012) 113 [arXiv:1203.1022] [INSPIRE].

[44] S. Moch, J.A.M. Vermaseren and A. Vogt, The three-loop splitting functions in QCD: the helicity-dependent case, Nucl. Phys. B 889 (2014) 351 [arXiv:1409.5131] [INSPIRE].

[45] G.P. Korchemsky, Asymptotics of the Altarelli-Parisi-Lipatov evolution kernels of parton distributions, Mod. Phys. Lett. A 4 (1989) 1257 [inSPIRE].

[46] J.M. Henn, A.V. Smirnov, V.A. Smirnov and M. Steinhauser, A planar four-loop form factor and cusp anomalous dimension in QCD, JHEP 05 (2016) 066 [arXiv:1604.03126] [INSPIRE].

[47] J. Henn, A.V. Smirnov, V.A. Smirnov, M. Steinhauser and R.N. Lee, Four-loop photon quark form factor and cusp anomalous dimension in the large- $N_{c}$ limit of QCD, JHEP 03 (2017) 139 [arXiv: 1612.04389] [INSPIRE].

[48] W.L. van Neerven and A. Vogt, NNLO evolution of deep inelastic structure functions: the nonsinglet case, Nucl. Phys. B 568 (2000) 263 [hep-ph/9907472] [INSPIRE].

[49] W.L. van Neerven and A. Vogt, NNLO evolution of deep inelastic structure functions: the singlet case, Nucl. Phys. B 588 (2000) 345 [hep-ph/0006154] [INSPIRE].

[50] W.L. van Neerven and A. Vogt, Improved approximations for the three loop splitting functions in QCD, Phys. Lett. B 490 (2000) 111 [hep-ph/0007362] [INSPIRE].

[51] J. Kalinowski, K. Konishi, P.N. Scharbach and T.R. Taylor, Resolving QCD jets beyond leading order: quark decay probabilities, Nucl. Phys. B 181 (1981) 253 [InSPIRE].

[52] J. Kalinowski, K. Konishi and T.R. Taylor, Jet calculus beyond leading logarithms, Nucl. Phys. B 181 (1981) 221 [INSPIRE]. 
[53] T. Munehisa, H. Okada, K. Kudoh and K. Kitani, Two loop anomalous dimensions of timelike cut vertices and scaling violation of fragmentation functions in QCD, Prog. Theor. Phys. 67 (1982) 609 [INSPIRE].

[54] A. Mitov and S.-O. Moch, QCD corrections to semi-inclusive hadron production in electron-positron annihilation at two loops, Nucl. Phys. B 751 (2006) 18 [hep-ph/0604160] [INSPIRE].

[55] O. Gituliar, Master integrals for splitting functions from differential equations in $Q C D$, JHEP 02 (2016) 017 [arXiv:1512.02045] [INSPIRE].

[56] A. Mitov, S. Moch and A. Vogt, Next-to-next-to-leading order evolution of non-singlet fragmentation functions, Phys. Lett. B 638 (2006) 61 [hep-ph/0604053] [INSPIRE].

[57] S. Moch and A. Vogt, On third-order timelike splitting functions and top-mediated Higgs decay into hadrons, Phys. Lett. B 659 (2008) 290 [arXiv:0709.3899] [INSPIRE].

[58] A.A. Almasy, S. Moch and A. Vogt, On the next-to-next-to-leading order evolution of flavour-singlet fragmentation functions, Nucl. Phys. B 854 (2012) 133 [arXiv:1107.2263] [INSPIRE].

[59] D.P. Anderle, F. Ringer and M. Stratmann, Fragmentation functions at next-to-next-to-leading order accuracy, Phys. Rev. D 92 (2015) 114017 [arXiv:1510.05845] [INSPIRE].

[60] NNPDF collaboration, V. Bertone, S. Carrazza, N.P. Hartland, E.R. Nocera and J. Rojo, A determination of the fragmentation functions of pions, kaons and protons with faithful uncertainties, Eur. Phys. J. C 77 (2017) 516 [arXiv:1706.07049] [InSPIRE].

[61] V.N. Gribov and L.N. Lipatov, Deep inelastic ep scattering in perturbation theory, Sov. J. Nucl. Phys. 15 (1972) 438 [Yad. Fiz. 15 (1972) 781] [InSPIRE].

[62] V.N. Gribov and L.N. Lipatov, $e^{+} e^{-}$pair annihilation and deep inelastic ep scattering in perturbation theory, Sov. J. Nucl. Phys. 15 (1972) 675 [Yad. Fiz. 15 (1972) 1218] [InSPIRE].

[63] M. Stratmann and W. Vogelsang, Next-to-leading order evolution of polarized and unpolarized fragmentation functions, Nucl. Phys. B 496 (1997) 41 [hep-ph/9612250] [INSPIRE].

[64] J. Blümlein, V. Ravindran and W.L. van Neerven, On the Drell-Levy-Yan relation to $O\left(\alpha_{s}^{2}\right)$, Nucl. Phys. B 586 (2000) 349 [hep-ph/0004172] [INSPIRE].

[65] Yu. L. Dokshitzer, G. Marchesini and G.P. Salam, Revisiting parton evolution and the large-x limit, Phys. Lett. B 634 (2006) 504 [hep-ph/0511302] [INSPIRE].

[66] Yu. L. Dokshitzer and G. Marchesini, $N=4$ SUSY Yang-Mills: three loops made simple(r), Phys. Lett. B 646 (2007) 189 [hep-th/0612248] [INSPIRE].

[67] B. Basso and G.P. Korchemsky, Anomalous dimensions of high-spin operators beyond the leading order, Nucl. Phys. B 775 (2007) 1 [hep-th/0612247] [INSPIRE].

[68] G. 't Hooft, Dimensional regularization and the renormalization group, Nucl. Phys. B 61 (1973) 455 [INSPIRE].

[69] W.A. Bardeen, A.J. Buras, D.W. Duke and T. Muta, Deep inelastic scattering beyond the leading order in asymptotically free gauge theories, Phys. Rev. D 18 (1978) 3998 [INSPIRE].

[70] C.G. Bollini and J.J. Giambiagi, Dimensional renormalization: the number of dimensions as a regularizing parameter, Nuovo Cim. B 12 (1972) 20 [INSPIRE]. 
[71] G. 't Hooft and M.J.G. Veltman, Regularization and renormalization of gauge fields, Nucl. Phys. B 44 (1972) 189 [INSPIRE].

[72] I. Bierenbaum, J. Blümlein and S. Klein, Mellin moments of the $O\left(\alpha_{s}^{3}\right)$ heavy flavor contributions to unpolarized deep-inelastic scattering at $Q^{2} \gg m^{2}$ and anomalous dimensions, Nucl. Phys. B 820 (2009) 417 [arXiv:0904.3563] [INSPIRE].

[73] P. Nogueira, Automatic Feynman graph generation, J. Comput. Phys. 105 (1993) 279.

[74] B. Ruijl, T. Ueda and J. Vermaseren, FORM version 4.2, arXiv:1707.06453 [INSPIRE].

[75] T. van Ritbergen, A.N. Schellekens and J.A.M. Vermaseren, Group theory factors for Feynman diagrams, Int. J. Mod. Phys. A 14 (1999) 41 [hep-ph/9802376] [inSPIRE].

[76] F. Herzog, B. Ruijl, T. Ueda, J.A.M. Vermaseren and A. Vogt, FORM, diagrams and topologies, PoS (LL2016) 073 [arXiv: 1608.01834] [INSPIRE].

[77] J.A.M. Vermaseren, The Minos database facility webpage, https://www.nikhef.nl/ form/maindir/others/minos/minos.html.

[78] S. Moch, J.A.M. Vermaseren and A. Vogt, $O n \gamma_{5}$ in higher-order QCD calculations and the NNLO evolution of the polarized valence distribution, Phys. Lett. B 748 (2015) 432 [arXiv: 1506.04517] [INSPIRE].

[79] B. Ruijl, T. Ueda, J.A.M. Vermaseren and A. Vogt, Four-loop QCD propagators and vertices with one vanishing external momentum, JHEP 06 (2017) 040 [arXiv:1703.08532] [INSPIRE].

[80] K.G. Chetyrkin and F.V. Tkachov, Infrared $R$ operation and ultraviolet counterterms in the MS scheme, Phys. Lett. B 114 (1982) 340 [INSPIRE].

[81] K.G. Chetyrkin and V.A. Smirnov, $R^{*}$ operation corrected, Phys. Lett. B 144 (1984) 419 [INSPIRE].

[82] F. Herzog and B. Ruijl, The $R^{*}$-operation for Feynman graphs with generic numerators, JHEP 05 (2017) 037 [arXiv: 1703.03776] [INSPIRE].

[83] F. Herzog, B. Ruijl, T. Ueda, J.A.M. Vermaseren and A. Vogt, The five-loop $\beta$-function of Yang-Mills theory with fermions, JHEP 02 (2017) 090 [arXiv: 1701.01404] [INSPIRE].

[84] F. Herzog, B. Ruijl, T. Ueda, J.A.M. Vermaseren and A. Vogt, On Higgs decays to hadrons and the R-ratio at $N^{4} L O$, JHEP 08 (2017) 113 [arXiv:1707.01044] [INSPIRE].

[85] D.J. Broadhurst, A.L. Kataev and C.J. Maxwell, Comparison of the Gottfried and Adler sum rules within the large- $N_{c}$ expansion, Phys. Lett. B 590 (2004) 76 [hep-ph/0403037] [INSPIRE].

[86] V.M. Braun, A.N. Manashov, S. Moch and M. Strohmaier, Three-loop evolution equation for flavor-nonsinglet operators in off-forward kinematics, JHEP 06 (2017) 037 [arXiv: 1703.09532] [INSPIRE].

[87] E. Remiddi and J.A.M. Vermaseren, Harmonic polylogarithms, Int. J. Mod. Phys. A 15 (2000) 725 [hep-ph/9905237] [inSPIRE].

[88] S. Moch and J.A.M. Vermaseren, Deep inelastic structure functions at two loops, Nucl. Phys. B 573 (2000) 853 [hep-ph/9912355] [INSPIRE].

[89] T. Lukowski, A. Rej and V.N. Velizhanin, Five-loop anomalous dimension of twist-two operators, Nucl. Phys. B 831 (2010) 105 [arXiv:0912.1624] [inSPIRE]. 
[90] V.N. Velizhanin, Results related with the calculations of the full five-loop anomalous dimension of twist-two operators in the planar $N=4 S Y M$ theory, webpage, http://thd.pnpi.spb.ru/ velizh/5loop/.

[91] R. Kirschner and L.N. Lipatov, Double logarithmic asymptotics and Regge singularities of quark amplitudes with flavor exchange, Nucl. Phys. B 213 (1983) 122 [INSPIRE].

[92] J. Blümlein and A. Vogt, On the behavior of nonsinglet structure functions at small $x$, Phys. Lett. B 370 (1996) 149 [hep-ph/9510410] [INSPIRE].

[93] A. Vogt et al., Progress on double-logarithmic large- $x$ and small-x resummations for (semi-)inclusive hard processes, PoS (LL2012) 004 [arXiv:1212.2932] [INSPIRE].

[94] J. Davies, C.H. Kom and A. Vogt, Resummation of small-x double logarithms in QCD: inclusive deep-inelastic scattering, to appear.

[95] A. Vogt, Resummation of small-x double logarithms in QCD: semi-inclusive electron-positron annihilation, JHEP 10 (2011) 025 [arXiv:1108.2993] [INSPIRE].

[96] C.H. Kom, A. Vogt and K. Yeats, Resummed small-x and first-moment evolution of fragmentation functions in perturbative QCD, JHEP 10 (2012) 033 [arXiv:1207.5631] [INSPIRE].

[97] V.N. Velizhanin, Generalised double-logarithmic equation in QCD, arXiv:1412.7143 [INSPIRE].

[98] S. Moch, J.A.M. Vermaseren and A. Vogt, Higher-order corrections in threshold resummation, Nucl. Phys. B 726 (2005) 317 [hep-ph/0506288] [INSPIRE].

[99] S. Moch and A. Vogt, Higher-order soft corrections to lepton pair and Higgs boson production, Phys. Lett. B 631 (2005) 48 [hep-ph/0508265] [INSPIRE].

[100] V. Ravindran, Higher-order threshold effects to inclusive processes in QCD, Nucl. Phys. B 752 (2006) 173 [hep-ph/0603041] [INSPIRE].

[101] T. Ahmed, M. Mahakhud, N. Rana and V. Ravindran, Drell-Yan production at threshold to third order in QCD, Phys. Rev. Lett. 113 (2014) 112002 [arXiv:1404.0366] [INSPIRE].

[102] J.A. Gracey, Anomalous dimension of nonsinglet Wilson operators at $O\left(1 / N_{f}\right)$ in deep inelastic scattering, Phys. Lett. B 322 (1994) 141 [hep-ph/9401214] [INSPIRE].

[103] B. Ruijl, Towards five loop calculations in $Q C D$, http://www.physik.uzh.ch/en/seminars/ttpseminar/HS2016.html, seminar of 6 December 2016.

[104] V. Ravindran, J. Smith and W.L. van Neerven, Two-loop corrections to Higgs boson production, Nucl. Phys. B 704 (2005) 332 [hep-ph/0408315] [INSPIRE].

[105] L.J. Dixon, L. Magnea and G.F. Sterman, Universal structure of subleading infrared poles in gauge theory amplitudes, JHEP 08 (2008) 022 [arXiv:0805.3515] [INSPIRE].

[106] A. Vogt, Efficient evolution of unpolarized and polarized parton distributions with QCD-PEGASUS, Comput. Phys. Commun. 170 (2005) 65 [hep-ph/0408244] [InSPIRE].

[107] T. Gehrmann and E. Remiddi, Numerical evaluation of harmonic polylogarithms, Comput. Phys. Commun. 141 (2001) 296 [hep-ph/0107173] [INSPIRE].

[108] J. Ablinger, J. Blümlein, M. Round and C. Schneider, Algebraic and numeric representations of harmonic polylogarithms, their generalizations and special numbers, DESY-13-064. 
[109] T. van Ritbergen, J.A.M. Vermaseren and S.A. Larin, The four loop $\beta$-function in quantum chromodynamics, Phys. Lett. B 400 (1997) 379 [hep-ph/9701390] [INSPIRE].

[110] E. Gardi and L. Magnea, Factorization constraints for soft anomalous dimensions in QCD scattering amplitudes, JHEP 03 (2009) 079 [arXiv:0901.1091] [INSPIRE].

[111] T. Becher and M. Neubert, On the structure of infrared singularities of gauge-theory amplitudes, JHEP 06 (2009) 081 [Erratum ibid. 11 (2013) 024] [arXiv:0903.1126] [INSPIRE].

[112] E. Gardi and L. Magnea, Infrared singularities in QCD amplitudes, Nuovo Cim. C32N5-6 (2009) 137 [Frascati Phys. Ser. 50 (2010)] [arXiv:0908.3273] [INSPIRE].

[113] V. Ahrens, M. Neubert and L. Vernazza, Structure of infrared singularities of gauge-theory amplitudes at three and four loops, JHEP 09 (2012) 138 [arXiv: 1208.4847] [INSPIRE].

[114] R.H. Boels, T. Huber and G. Yang, The four-loop non-planar cusp anomalous dimension in $N=4 S Y M$, arXiv: 1705.03444 [INSPIRE].

[115] A. Grozin, J.M. Henn, G.P. Korchemsky and P. Marquard, The three-loop cusp anomalous dimension in QCD and its supersymmetric extensions, JHEP 01 (2016) 140 [arXiv: 1510.07803] [INSPIRE].

[116] M. Czakon, The four-loop QCD $\beta$-function and anomalous dimensions, Nucl. Phys. B 710 (2005) 485 [hep-ph/0411261] [INSPIRE].

[117] A. Grozin, Leading and next-to-leading large- $N_{f}$ terms in the cusp anomalous dimension and quark-antiquark potential, PoS (LL2016) 053 [arXiv: 1605.03886] [INSPIRE].

[118] R.N. Lee, A.V. Smirnov, V.A. Smirnov and M. Steinhauser, The $n_{f}^{2}$ contributions to fermionic four-loop form factors, Phys. Rev. D 96 (2017) 014008 [arXiv:1705.06862] [INSPIRE].

[119] J.C. Collins and R.J. Scalise, The renormalization of composite operators in Yang-Mills theories using general covariant gauge, Phys. Rev. D 50 (1994) 4117 [hep-ph/9403231] [INSPIRE].

[120] Z. Bajnok, R.A. Janik and T. Lukowski, Four loop twist two, BFKL, wrapping and strings, Nucl. Phys. B 816 (2009) 376 [arXiv:0811.4448] [INSPIRE].

[121] A.V. Kotikov, L.N. Lipatov, A. Rej, M. Staudacher and V.N. Velizhanin, Dressing and wrapping, J. Stat. Mech. 10 (2007) P10003 [arXiv:0704.3586] [INSPIRE].

[122] J.A.M. Vermaseren, Axodraw, Comput. Phys. Commun. 83 (1994) 45 [inSPIRE].

[123] D. Binosi and L. Theussl, JaxoDraw: a graphical user interface for drawing Feynman diagrams, Comput. Phys. Commun. 161 (2004) 76 [hep-ph/0309015] [INSPIRE]. 